\title{
Atmospheric Electron Neutrinos in the MINOS Far Detector
}

\author{
A THESIS \\ SUBMITTED TO THE FACULTY OF THE GRADUATE SCHOOL \\ OF THE UNIVERSITY OF MINNESOTA \\ BY
}

Benjamin Phillip Speakman

IN PARTIAL FULFILLMENT OF THE REQUIREMENTS

FOR THE DEGREE OF

Doctor Of Philosophy

May, 2007 
(C) Benjamin Phillip Speakman 2007

ALL RIGHTS RESERVED 


\title{
Atmospheric Electron Neutrinos in the MINOS Far Detector by Benjamin Phillip Speakman
}

\begin{abstract}
Neutrinos produced as a result of cosmic-ray interactions in the earth's atmosphere offer a powerful probe into the nature of this three-membered family of low-mass, weakly-interacting particles. Ten years ago, the Super-Kamiokande Experiment has confirmed [1] earlier indications that neutrinos undergo lepton-flavor oscillations during propagation, proving that they are massive contrary to the previous Standard Model assumptions. The Soudan Underground Laboratory, located in northern Minnesota, was host to the Soudan2 Experiment, which has made important contributions to atmospheric neutrino research. This same lab has more recently been host to the MINOS far detector, a neutrino detector which serves as the downstream element of an accelerator-based long-baseline neutrino-oscillation experiment.

This thesis has examined 418.5 live days of atmospheric neutrino data (fiducial exposure of 4.18 kton-years) collected in the MINOS far detector prior to the activation of the NuMI neutrino beam, with a specific emphasis on the investigation of electron-type neutrino interactions. Atmospheric neutrino interaction candidates have been selected and separated into showering or track-like events. The showering sample consists of 89 observed events, while the track-like sample consists of 112 observed events. Based on the Bartol atmospheric neutrino flux model of Barr et al. 2] plus a Monte Carlo (MC) simulation of interactions in the MINOS detector, the expected yields of showering and track-like events in the absence of neutrino oscillations are $88.0 \pm 1.0$ and $149.1 \pm 1.0$ respectively (where the uncertainties reflect only the limited MC statistics). Major systematic uncertainties, especially those associated with the flux model, are cancelled by forming a double ratio of these observed and expected yields:
\end{abstract}

$$
R_{t r k / s h w}^{\text {data }} / R_{\text {trk } / \text { shw }}^{M C}=0.74_{-0.10}^{+0.12}(\text { stat. }) \pm 0.04 \text { (syst.) }
$$

This double ratio should be equal to unity in the absence of oscillations, and the value above disfavors null oscillation with $96.0 \%$ confidence. In addition, the showering sample can be used to measure the atmospheric neutrino flux. Based on the analysis presented in this thesis, the Bartol flux should be scaled by a factor of:

$$
\left.S_{\text {atm }}=1.08 \pm 0.12 \text { (stat. }\right) \pm 0.08 \text { (syst.) }
$$

This is larger than, but consistent with, a measurement at the same location by the Soudan2 Experiment [3] of $S_{a t m}=0.91 \pm 0.07$. 


\section{Acknowledgements}

There are many people that have earned my gratitude for their contribution to my time in graduate school. To my family, many thanks to my parents and siblings for enduring my childhood and your continuing support over the years. To my incoming classmates, thanks to Chris Stepaniak, Dave Engebretson, and many other for those wonderful first years.

Thanks to all of the physics and astronomy professors at the University of Minnesota, especially to Roger Jones, Earl Peterson, and Benjamin Bayman and Ken Heller whose masterful teaching inspired and cultivated my love of physics. Also thanks the Peter Litchfield and Keith Ruddick for their significant editing contribution to this thesis.

Thanks all of the personnel at the Soudan Underground Lab for their amazing job in building and maintaining the detector. Bill Miller, Jerry Meier, and many others are owed an immense debt of gratitude from myself and the entire MINOS collaboration.

Thanks to my office mates and fellow MINOS graduate students Jeremy, Dipu, Sujeewa, Erik, Eric, Emily, and Bernie for lightening (and in some cases occupying a significant fraction of) my time in graduate school. Thanks to the Minnesota MINOS postdocs Hugh, Jeff, Leon, Sue, David, and Kregg for helping to understand everything about our experiment.

Thanks to the Cambridge group for their hospitality and for everything they have done with the atmospheric neutrino analysis, especially Pat Ward who laid the foundation for showering neutrino analysis. I can't imagine ever having graduated without the unbelievable efforts of John Chapman, Andy Blake, Caius Howcroft, Pat Ward, and Mark Thomson.

Thanks to my loving wife Siri, for enduring the countless times that I had just "one more year left" of graduate school, for help editing this thesis. Thanks to Brad, Dot, and Hamilton for maintaining my sanity.

Finally thanks to my advisor Jon Urheim for his continuing support through my overly long and storied graduate career. I have no doubt that one day there will be songs written about his herculean patience. 


\section{Dedication}

To my caring wife, and our dogs.

Tiger got to hunt, Bird got to fly; Man got to sit and wonder, "Why, why, why?"

Tiger got to sleep, Bird got to land; Man got to tell himself he understand

Kurt Vonnegut - Cat's Cradle 


\section{Contents}

Abstract i

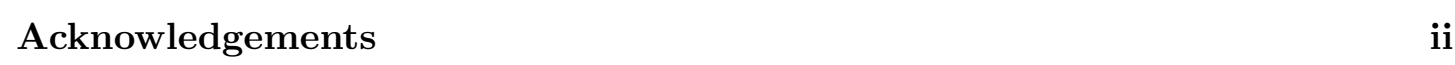

Dedication iii

List of Tables viii

List of Figures $\quad$ x

1 Introduction 1

2 Phvsics of Neutrinos 4

2.1 A Brief Historv of the Neutrino . . . . . . . . . . . . . 4

2.1.1 Missing Energy and a New Particle . . . . . . . . . . . . . 4

2.1.2 Early Neutrino Detection . . . . . . . . . . . . . 6

2.1.3 Measuring the Neutrino Mass . . . . . . . . . . . . . . 99 9

2.1 .4 Neutrino Flavor . . . . . . . . . . . . . . . . . . 10

2.2 Interaction of Neutrinos with Matter . . . . . . . . . . . . . . . 12

2.3 Neutrino Oscillation . . . . . . . . . . . . . . . . . . 14

2.3 .1 Two Neutrino Oscillation . . . . . . . . . . . . . 18

2.3 .2 Three Neutrino Oscillation . . . . . . . . . . . . . 19

2.4 Experimental Status of Neutrino Oscillation . . . . . . . . . . . . . 22

2.4 .1 Solar Neutrino Anomaly . . . . . . . . . . . . . . . 23

2.4 .2 Atmospheric Neutrino Anomaly . . . . . . . . . . . . . . 28 
2.4 .3 Reactor Neutrino Oscillation Experiments . . . . . . . . . . . 32

2.4.4 Accelerator Beam Neutrino Oscillation Experiments . . . . . . . 35

2.4 .5 Neutrino Oscillation Experimental Status . . . . . . . . . . 38

3 MINOS Experiment $\quad 40$

3.1 NuMI Beam . . . . . . . . . . . . . . . . . . . . . 42

3.2 MINOS Detectors . . . . . . . . . . . . . . . . . . . 45

3.2 .1 Near Detector . . . . . . . . . . . . . . . . . . . . . 45

$3.2 .2 \quad$ Far Detector $\ldots \ldots \ldots \ldots \ldots$. . . . . . . . . . . . . . . . . . .

4 Monte Carlo $\quad 57$

4.1 Incident Particle Flux . . . . . . . . . . . . . . . . . . . 58

4.1 .1 Atmospheric Neutrinos . . . . . . . . . . . . . . . . 58

4.1 .2 Cosmic-Rav Muons . . . . . . . . . . . . . . . . . 60

4.2 Particle Interactions with Detector Material . . . . . . . . . . . . . . 61

4.3 Active Detector Simulation . . . . . . . . . . . . . . . . . 63

4.3.1 Scintillator Strips and WLS Fiber . . . . . . . . . . . 63

4.3.2 Photo-multiplier Tubes and Electronics Response . . . . . . . . . 64

5 Event Reconstruction $\quad 65$

5.1 Noise Filter and Digit Reconstruction $\ldots \ldots \ldots$. . . . . . . . 67

5.2 Digit Reconstruction . . . . . . . . . . . . . . . . . . . 68

5.2 .1 Energy Response Calibration . . . . . . . . . . . 68

5.2 .2 Timing Response Calibration . . . . . . . . . . . . 70

5.3 Demultiplexing . . . . . . . . . . . . . . . . . . . . . . . 70

5.4 Construction of Tracks and Showers _ . . . . . . . . . . . . . 74

5.5 Direction and Energy Reconstruction . . . . . . . . . . . . . . . . 80

6 Data Analysis Strategy $\quad 86$

6.1 Analvsis Strategv . . . . . . . . . . . . . . . . 86

6.2 Data Set used in Analvsis . . . . . . . . . . . . . . . . . 90

6.3 Monte Carlo Sets used in Analvsis . . . . . . . . . . . . . . . . . . . . . 91

6.3 .1 Atmospheric Neutrinos . . . . . . . . . . . . . . . . . . 91 
6.3.2 Cosmic-Rav Muons . . . . . . . . . . . . . . . . . . . . . . 93

6.3 .3 Rock Neutrons . . . . . . . . . . . . . . . . . . . 96

6.4 Data Qualitv and Event Pre-Selection . . . . . . . . . . . . . . 97

6.4.1 Cambridge Filter . . . . . . . . . . . . . . . . . . . . . . 98

6.4.2 Hardware Based Selections . . . . . . . . . . . . . . . 100

6.5 Clean Analvtic Set . . . . . . . . . . . . . . . . . . . . 102

7 Event Selection 106

7.1 Track-like and Showering Sample Division . . . . . . . . . . . . . . 107

7.2 Vertex Containment . . . . . . . . . . . . . . . . . . . 108

7.3 Event Classification . . . . . . . . . . . . . . . . . . . . . 112

7.4 Shower Selection . . . . . . . . . . . . . . . . . . . . . 114

7.4 .1 Event Qualitv . . . . . . . . . . . . . . . . . . . . 115

7.4.2 Shower Quality . . . . . . . . . . . . . . . . . . . . . . . . . . 119

7.4 .3 Shower Trace . . . . . . . . . . . . . . . . . . . . . . . 120

7.4.4 Shower Topologv . . . . . . . . . . . . . . . . . . . . . . . 122

7.4.5 Shower Selection Results . . . . . . . . . . . . . . . . 127

7.5 Track Selection . . . . . . . . . . . . . . . . . . . . 130

7.5.1 Track and Event Qualitv _. . . . . . . . . . . . . . . 130

7.5.2 Fullv Contained and Partiallv Contained Downward Tracks . . . 135

7.5.3 Partiallv-Contained Upward Tracks . . . . . . . . . . . . . . 142

7.6 Veto Shield . . . . . . . . . . . . . . . . . . . . . . . . 146

7.6 .1 Shield Inefficiency $\ldots \ldots \ldots$

7.6.2 Shield Efficiency . . . . . . . . . . . . . . . . . . . . . 151

7.7 Final Data Set . . . . . . . . . . . . . . . . . . . . 152

7.7 .1 Double Ratio . . . . . . . . . . . . . . . . . 153

7.7.2 Oscillation Independent Flux Measurement . . . . . . . . . 158

8 Oscillation Analysis 161

8.1 Neutrino Oscillation Procedure . . . . . . . . . . . . . . . . . . 161

8.2 Frequentist Double Ratio Oscillation Analysis . . . . . . . . . . . . . . 164

8.3 Likelihood Oscillation Analvsis . . . . . . . . . . . . . . . . 166

8.4 Projected Sensitivity . . . . . . . . . . . . . . . . . . . 173 
9 Conclusion and Discussion $\quad 175$

$\begin{array}{lr}\text { Appendix A. Selected Events } & 177\end{array}$

A.1 Selected Showers . . . . . . . . . . . . . . . . . . . . . 177

A.2 Selected Tracks . . . . . . . . . . . . . . . . . . . 180

A.2.1 Fully Contained . . . . . . . . . . . . . . . . . . 181

A.2.2 Partiallv Contained Downward . . . . . . . . . . . . 183

A.2.3 Partially Contained Upward . . . . . . . . . . . . . . . . . . . 184

Appendix B. Notes on Errors and Coverages 186

B.1 Double Ratio Coverage . . . . . . . . . . . . . . . . . . 186

B.2 Analytic Maximization of Likelihood with Scaled Contributions . . . . . 191 


\section{List of Tables}

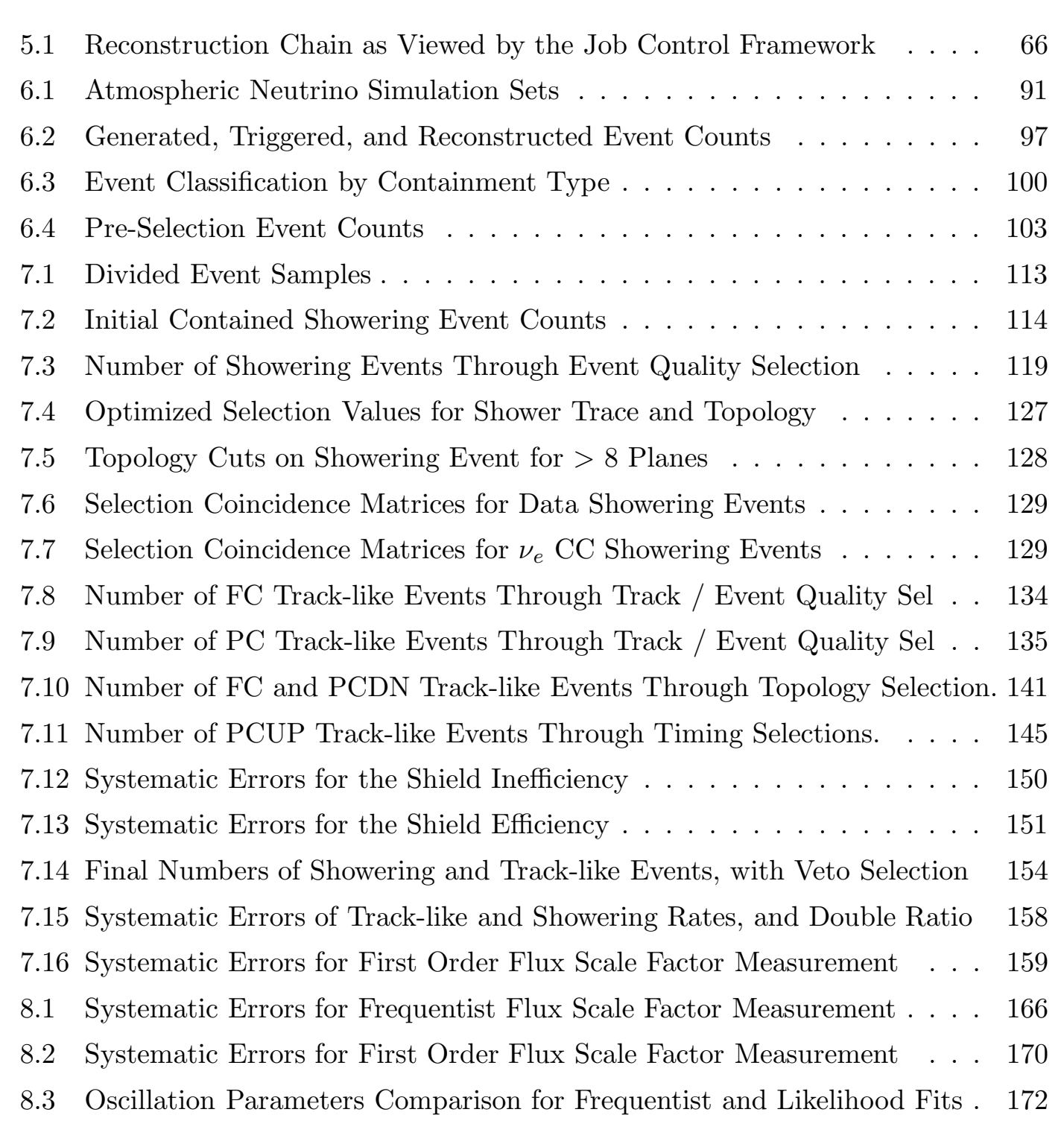


A.1 Selected Fully Contained Showers . . . . . . . . . . . . . . . . . . . . . 177

A.2 Selected Fully Contained Tracks _. . . . . . . . . . . . . . . . . . . . . 181

A.3 Selected Partially-Contained Downward Tracks . . . . . . . . . . . . . . 183

A.4 Selected Partially-Contained Upward Tracks . . . . . . . . . . . . . . . . 184 


\section{List of Figures}

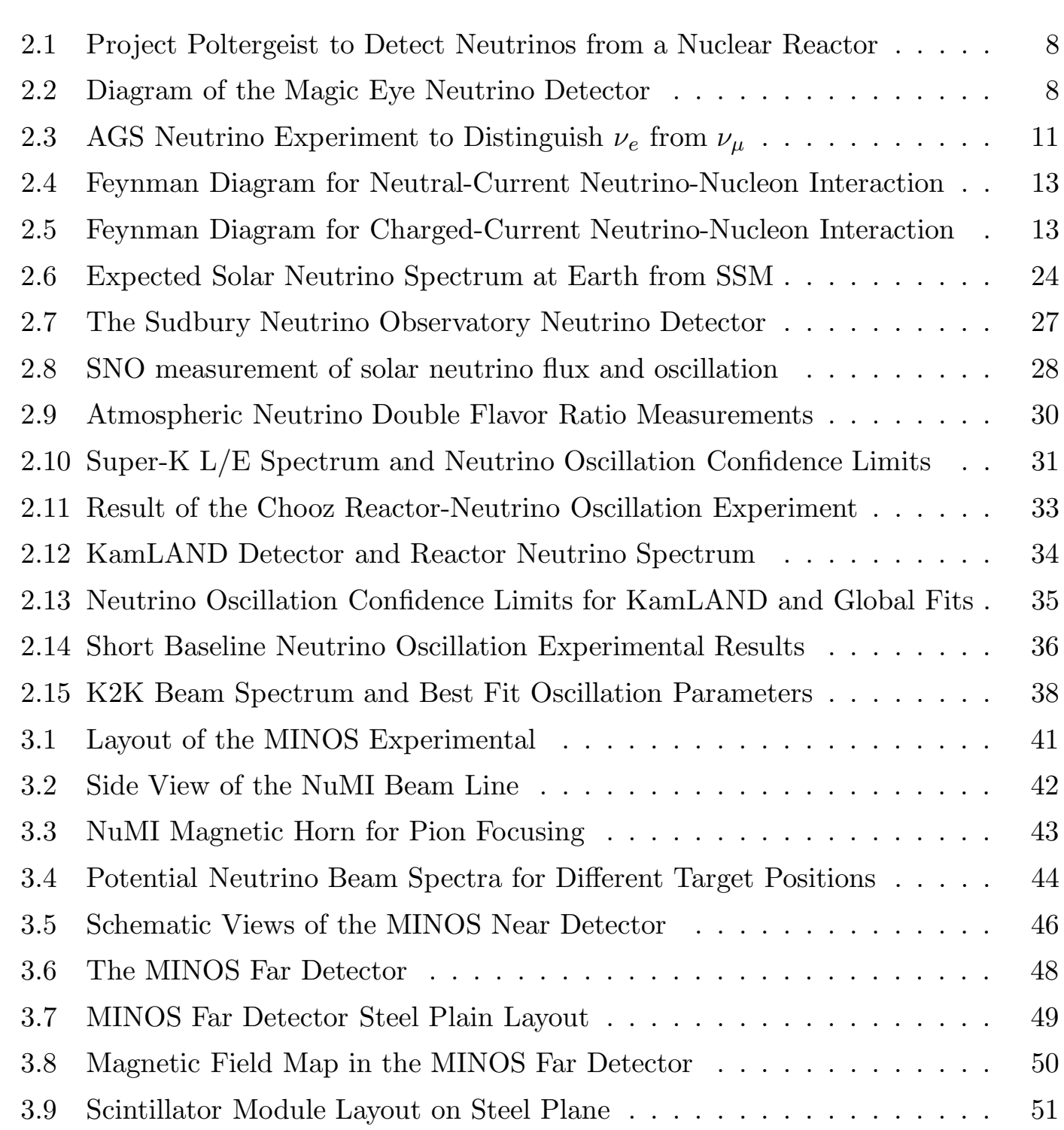


3.10 Extension Manifold on 20 Strip Scintillator Module . . . . . . . . . . . 52

3.11 Veto Shield Desion and Example Event Tagoed in the Veto Shield . . . 53

3.12 Fiber Multiplexing Box and 16 Pixel Photo-multiplier Tubes . . . . . . 54

4.1 Atmospheric Neutrino Flux for Bartol Model. Comparison 1D to 3D . . 59

4.2 Atmospheric Neutrino Flux Uncertaintv for the Bartol Model . . . . . . 60

5.1 Schematic View of the MINOS Light Iniection Svstem . . . . . . . . 69

5.2 Optical Summing Pattern on Opposing Pixels . . . . . . . . . . . . 71

5.3 Demultiplexing Performance for Neutrinos and Cosmic-rav Muons . . . 73

5.4 Demultiplexing Performance versus Digits Ouantities . . . . . . . . 73

5.5 Efficiency for Reconstruction Tracks and Showers . . . . . . . . . 75

5.6 Shower Completeness and Puritv Performance . . . . . . . . . . . 77

5.7 Shower Vertex Finding Performance _. . . . . . . . . . . . . . 77

5.8 Track Completeness and Puritv Performance $\ldots \ldots \ldots \ldots \ldots$

5.9 Track Vertex Finding Performance _ . . . . . . . . . . . . . . 78

5.10 Shower Energy Reconstruction Performance for $\nu_{\rho}$ CC Interactions . . . 82

5.11 Shower Energy Reconstruction Performance for $\nu$ NC Interactions $\ldots 82$

5.12 Track Momentum Reconstruction Performance for FC Tracks . . . . . 82

5.13 Track Momentum Reconstruction Performance for PC Tracks _. . . . . 83

5.14 Shower Direction Performance for $\nu_{e} \mathrm{CC}$ and $\nu \mathrm{NC}$ Interactions . . . . 85

5.15 Track Direction Performance _... . . . . . . . . . . . 85

6.1 Interacting Neutrino Enerov Spectra for Generated to Reconstructed Sets 92

6.2 Muon Rates Over Data Exposure . . . . . . . . . . . . . . . . . . . 94

6.3 Simulated Cosmic-Rav Muon Spectra … . . . . . . . . 95

6.4 Good Planes Bv Event and Views _... . . . . . . . . . . . . 98

6.5 Fraction of Sincle-Ended Strins in and Events . . . . . . . . . . . 102

6.6 The average data rate for each dav at several selection stages $\ldots . . .103$

6.7 Neutrino Energv Progression to Cleaned . . . . . . . . . . . . . . . . 104

7.1 Number of Reconstructed Tracks or Shower . . . . . . . . . . . . 107

7.2 Track and Shower Vertex Depths . . . . . . . . . . . . . . . . . 109

7.3 Track and Shower Distance to Plane Face _ . . . . . . . . . . . . 110

7.4 Track and Shower Radial Vertex from Center . . . . . . . . . . . . 112

7.5 Energy Fraction outside Single Supermodule Fiducial Volume . . . . . . 115 


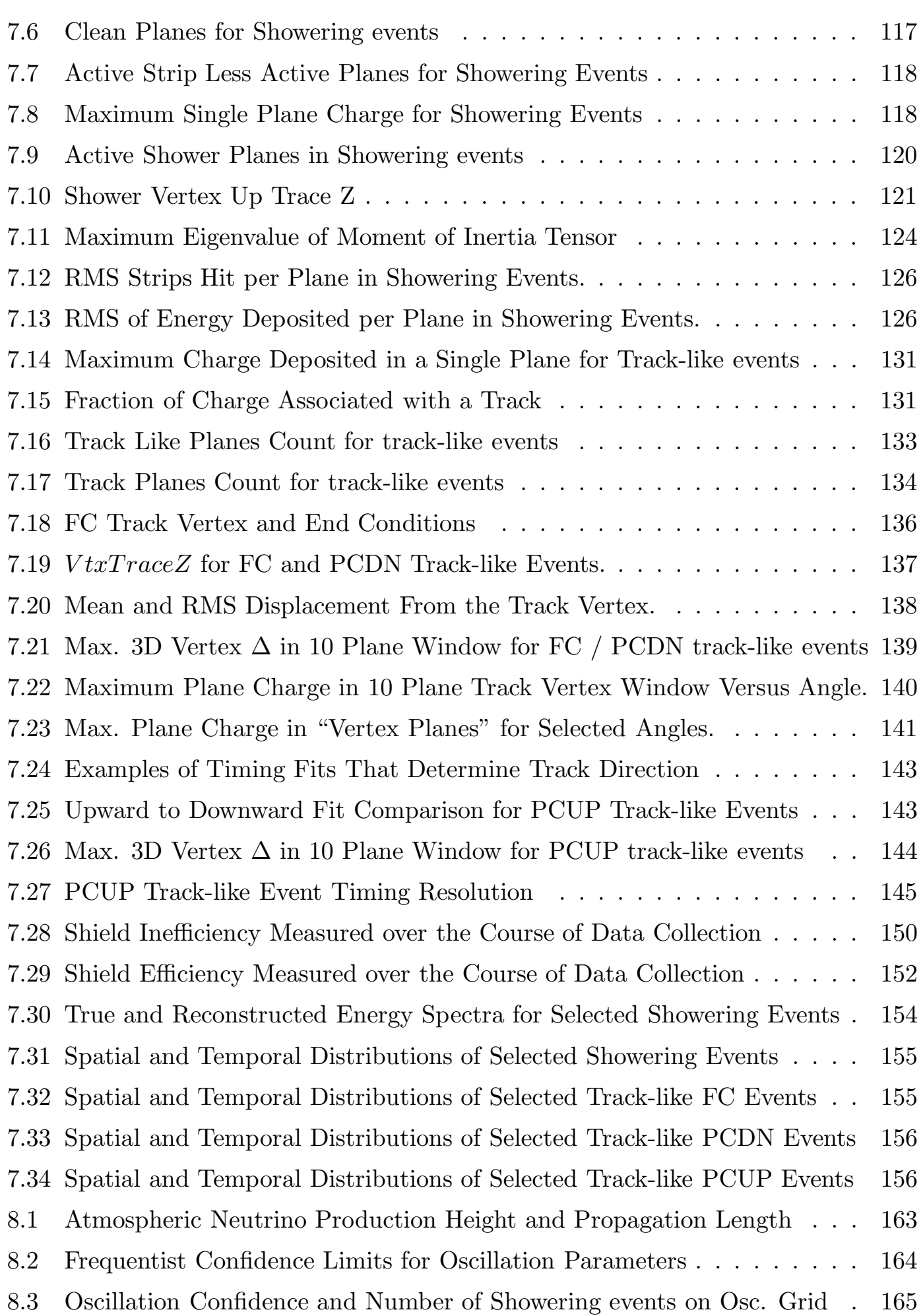


8.4 Weakly Penalized Flux Scale Likelihood Fit . . . . . . . . . . . . . . . 168

8.5 Flux Scale Measurement with Likelihood Fit _. . . . . . . . . . . . . . 169

8.6 Maximal Mixing Slices in Oscillation Parameters . . . . . . . . . . . . . 171

8.7 MINOS Best $\Delta m^{2}=0.00274 \mathrm{eV}^{2}$ Slices in Oscillation Parameters . . . . 171

8.8 Projected Double Ratio Sensitivitv . . . . . . . . . . . . . . . . . . 173

B.1 Poisson Fluctuated Number of Tracks and Showers . . . . . . . . . . . 188

B.2 Poisson Fluctuated Double Ratio Distributions _ . . . . . . . . . . . . 188

B.3 Poisson and binomial Fluctuated Number of Tracks and Showers . . . . 189

B.4 Poisson and binomial Fluctuated Double Ratio Distributions . . . . . . 190 


\section{Chapter 1}

\section{Introduction}

Compelling evidence has been put forward in the last few decades that the neutrino is a massive particle, contrary to early Standard Model assumptions. Since its inception in the 1930s, up to its first direct observation in the 1950s, the neutrino has always been a difficult particle to study for one very significant reason; compared to other particles it is extremely difficult to detect because it only interacts via the weak force. For this reason, the detection of neutrinos has always relied on either an intense source or a significant amount of target mass.

Neutrinos that are experimentally examined can be divided into two categories: artificial neutrinos from a man-made source (e.g. a nuclear reactor or a neutrino beam), or naturally occurring neutrinos (e.g. atmospheric or solar neutrinos). Artificial neutrino sources have the benefit of control over the neutrino intensity and energy, which allows precision probes to be made in to the nature of neutrinos. However, the investigation of natural neutrinos spares one the high cost of neutrino generation while also serving as a probe into the nature of the phenomena that lead to their creation.

Solar neutrinos were originally studied to confirm the predicted model of nuclear processes that cause the sun to shine. The nuclear fusion chains in the core of the sun are expected to produce neutrinos in addition to large amounts of energy from photons. The anomalously low rate of solar neutrino interactions was the first clue towards flavor oscillation of massive neutrinos. Several experiments over the years have produced more compelling evidence that the deficit in solar neutrino interactions is the result of neutrino oscillation. 
Atmospheric neutrinos are the result of high energy cosmic-rays hitting the upper atmosphere, and creating a spray of short-lived particles that decay into neutrinos. As with solar neutrinos, early studies of atmospheric neutrinos revealed anomalously low interaction rates, leading to the hypothesis that neutrino oscillation effects were present here as well. For this thesis, atmospheric electron-type neutrinos $\left(\nu_{e}\right)$ are investigated using the deep underground MINOS Far Detector.

The analysis in this thesis builds upon a previous atmospheric neutrino analysis 4]. This previous analysis exclusively used track-like neutrino interactions, which are composed primarily of charged-current (CC) interactions of muon-type neutrinos $\left(\nu_{\mu}\right)$. Both the previous analysis, and that presented in this thesis, have used the Bartol atmospheric neutrino flux model presented by Barr et. al. [5]. The Bartol model claims a $20 \%$ uncertainty on the overall flux [6], an uncertainty which has a significant impact on an oscillation analysis. The atmospheric neutrino anomaly is based on a deficit of neutrino interactions, and a downward $20 \%$ shift in the overall flux could partially account for this deficit.

To accurately predict the atmospheric neutrino flux, an experiment must measure the interaction rate in a way that is insensitive to neutrino oscillations. Previous experiments have used two such interaction rates to measure the overall flux: the downward $\nu_{\mu}$ CC interaction rate, or the overall $\nu_{e}$ CC interaction rate. The Bartol model claims uncertainties of $\sim 1 \%$ for the ratios of $\nu_{e} / \nu_{\mu}$ and $\nu_{\mu}$ up/down. The approach has been taken in this thesis to identify neutrino oscillation with the "atmospheric neutrino flavor double ratio". A selected set of atmospheric neutrino candidates has been divided into showering and track-like events, which are rich in $\nu_{e} \mathrm{CC}$ and $\nu_{\mu} \mathrm{CC}$ interactions respectively. The flavor ratio $\left(R_{t r k / s h w}^{d a t a} / R_{t r k / s h w}^{M C}\right)$ is minimally sensitive to the $20 \%$ uncertainty in the atmospheric neutrino flux, and has been used in other experiments to identify the deficit in the $\nu_{\mu}$ atmospheric neutrino component. The $\nu_{e}$ component of atmospheric neutrinos has agreed with flux model predictions, and so a flavor ratio less than 1.0 has been used as evidence of lepton-flavor oscillation of massive-neutrinos.

The Soudan2 experiment was the predecessor of underground high-energy physics in the Soudan mine. Soudan2 used the observed yield of $\nu_{e}$ interaction candidates to normalize the flux model used in their simulation of the $\nu_{m} u$ energy and angular distributions [3], and found that the Bartol atmospheric-neutrino flux model should be 
scaled by $0.88 \pm 0.07$ to match their data. The aforementioned $\nu_{\mu} \mathrm{CC}$ analysis [4] used the normalization information from the Soudan2 experiment, and scaled the Bartol flux model by the same factor. The analyses presented in this thesis have measured the rate of $\nu_{e}$ interactions in order to resolve the atmospheric neutrino flux in the same manner as the Soudan2 experiment.

This thesis presents the investigation of atmospheric neutrinos in the MINOS Far Detector in the following progression:

- Chapter 2 briefly presents the history of the neutrino, the physics of neutrino interactions and oscillation, and the experimental status of neutrino oscillation.

- In Chapter 3 the MINOS project in outlined, with a special emphasis on the Far Detector.

- Chapter 4 describes the Monte Carlo process used to generate the simulated sets used in the analysis.

- Chapter 5 follows the chain of reconstruction software used to obtain meaningful physics result from the events in the detector.

- Chapter 6 hashes out the strategy for analysis and presents the data and simulated sets that will be used in the analysis.

- Chapter 7 demonstrates the implementation of the event selection processes by which the atmospheric neutrino interactions are isolated from the cosmic-ray muon background.

- In Chapter 8 the selected events from Chapter 7 are joined to measure neutrino oscillation parameters, and the atmospheric neutrino flux.

- Chapter 9 presents a final discussion of the analyses presented in the thesis. 


\section{Chapter 2}

\section{Physics of Neutrinos}

The neutrino is 73 years old in the human consciousness. Spectacular advances have been made over those years in the understanding of the physical properties of this particle, but much of its nature is still not known. It is the goal of this thesis to understand the neutrino's mass and quantum mixing of the three massive neutrino states. To provide background for the research described here, the history of the neutrino is presented briefly in this chapter, along with current experimental results regarding the oscillation of neutrinos.

\subsection{A Brief History of the Neutrino}

\subsubsection{Missing Energy and a New Particle}

Over one-hundred years ago, the science of radioactivity was introduced as a relative newcomer to the ancient field of physics. The originators of this science (Henri Becquerel, along with Marie and Pierre Curie) have the honor of having basic units of nuclear radioactivity named for them $(1$ Becquerel $=1$ decay $/$ second, 1 Curie $=3.7 \times$ $10^{10}$ decays / second $=$ the activity of 1 gram of radium). These physicists determined that natural radioactive decay occurs when an element spontaneously converts itself to another element, emitting energetic particles in the process.

With particle detection instruments that seem primitive by today's standards, physicists in the early part of the $20^{\text {th }}$ century discovered that nuclear decay produced three 
distinct particles that were affected differently by a magnetic field. The particles whose path bent in two different directions were called $\alpha$ and $\beta$ particles, while those particles that were unaffected by the magnetic field were named $\gamma$ particles.

The small masses of the $\alpha$ and $\beta$ particles were insufficient to account for the difference in the mass of the atom before and after radioactive decay. A solution to this problem arrived in 1905 via Albert Einstein [7], with the famous energy mass equivalence formula $\mathrm{E}=\mathrm{m} \mathrm{c}^{2}$. The fundamental principle of conservation of energy still held when measuring the energy of $\alpha$ decay particles. The radioactive $\beta$ decay of some elements however was not producing spectral results that were consistent with energy conservation.

$$
{ }_{83} R a E^{210} \rightarrow{ }_{84} R a F^{210}+\beta^{-}
$$

In 1914 James Chadwick found that the energy spectrum of $\beta$ decay particles from the RadiumE isotope did not peak at the energy equivalent to the mass difference of the radioactive decay atoms. The energy spectrum was instead spread out, and peaked at an energy lower than expected. The $\beta$ decay process was, at the time, understood to be the reaction in Equation 2.1. The electron was the only decay particle emitted from the atom, so it would have to carry the energy away. In addition to the trouble with the $\beta$ decay spectrum, the concept of the $\beta$ decay was problematic.

Rutherford discovered with an $\alpha$ scattering experiment that the atom's positive charge was concentrated in a small area at the center of the atom (the nucleus). The positive nucleus of the atom would have an integral spin value, and the radioactive decay from one element to another would demand an integral change in spin. The $\beta$ particle has a spin of $1 / 2$, and so a single $\beta$ decay would not account for the change in spin.

To reconcile this, Wolfgang Pauli is credited with the solution that an additional particle must be ejected from the nucleus during the $\beta$ decay process. This particle would have to have very little mass, and no electrical charge to have eluded detection. Pauli and Enrico Fermi worked together trying to understand this possibility, but Pauli was reluctant to claim that the process demanded an additional particle that would be impossible to detect.

When Chadwick discovered the neutron in 1932 [8], the atom was once again redefined, this time as a nucleus of protons and neutrons surrounded by a cloud of electrons. 
The number of protons and electrons would have be equal to produce neutral atom, and that number defined the atom's element class. Two years later, Pauli published a paper that officially proposed the existence of the neutrino, and Fermi published a paper that described a new theory for the $\beta$ decay process that included a neutrino. The reaction in Equation 2.1] was reformulated to the reaction in Equation 2.2. Fermi postulated that, inside of the nucleus, the $\beta$-decay process was the decay of a neutron to a proton, a $\beta$ particle, and an anti-neutrino (Equation 2.3) 9].

$$
\begin{aligned}
{ }_{83} R a E^{210} & \rightarrow{ }_{84} R a F^{210}+\beta^{-}+\bar{\nu} \\
N^{0} & \rightarrow P^{+}+\beta^{-}+\bar{\nu}
\end{aligned}
$$

As of 1934, the neutrino was partially accepted in the particle physics community as a reality. Complete acceptance would not happen until there was a way to detect a neutral low mass particle. Five years later, in the fall of 1939, Adolf Hitler and his Nazi army invaded Poland, and the second world war put the esoteric research goals of neutrino physics on the shelf, while more pertinent uses of nuclear physics were sought from the particle physics community at large. The first step in the creation of the atomic bomb was to produce controlled, sustainable, nuclear reactions. The development of these nuclear reactors during the Manhattan Project made the first direct detection of neutrinos a possibility.

\subsubsection{Early Neutrino Detection}

In addition to producing a vast amount of energy in the form of neutrons and $\gamma$ particles, nuclear reactors were also a powerful source of neutrinos. Theorists predicted that the neutrino would have to be very non-interactive in order to have escaped detection, and so the experimentalists saw the statistical opportunity offered by the abundance of the neutrino from these reactors. Clyde Cowan and Frederick Reines conceived a method to detect neutrinos in an experiment called Project Poltergeist (Figure 2.1). The process that they sought to exploit for neutrino detection was related to the neutron decay mechanism in Equation 2.3. Rather than a neutron decaying to a proton, $\beta$, and an anti-neutrino, an anti-neutrino may interact with a proton and result in a neutron and an anti- $\beta$ particle in process then called inverse $\beta$-decay (Equation 2.4). The resulting 
anti- $\beta$ (or positron) would annihilate to produce two $\gamma$ particles, and the neutron would have to be captured in order produce a detectable signal.

$$
P^{+}+\bar{\nu} \rightarrow \beta^{+}+N^{0}
$$

To detect inverse $\beta$-decay, Cowan and Reines constructed a device called the "Magic Eye" that would try to identify the signature of a positron annihilation accompanied by a prompt neutron. The Magic Eye tank was filled with water, as a target for the neutrino interactions. In order to make the neutron produce a detectable signal, the water was doped with Cadmium Oxalate salt. The $\mathrm{Cd}^{+}$nuclei in the water would capture the neutron after some short time $(\sim 10 \mu \mathrm{sec})$, and emit a batch of $\gamma$ particles. The delay between the annihilation signal and the neutron capture signal defined the signature of an anti-neutrino interaction. Photomultiplier tubes with liquid scintillator caught the two $\gamma$ signals, and the electronics watched for the $10 \mu$ sec delayed pulses.

Project Poltergeist first tested this method in 1953 at the Hanford reactor facility, but the background rate of accidentals was to large for the signal to be observable. In 1956, the same test was performed again at the Savannah River reactor, and this time they were successful in detecting the expected neutrino signal. They found the cross-section of the neutrino-proton interaction to be $\sigma=(11 \pm 2.6) \times 10^{-44} \mathrm{~cm}^{2}$.

Cowan and Reines detected anti-neutrinos with Project Poltergeist, but the detection of neutrinos was not possible by this method. The reactors of the day were essentially anti-neutrino factories. In addition, the inverse $\beta$ decay process for a neutrino in Equation 2.5 has a less distinguishable signature than that process in Equation 2.4. In 1946, Bruno Pontecorvo suggested that the neutrino-proton interaction could be detected by observing chemical impurities over time in a chemically pure sample [11]. He suggested that a measurement could be made of the gradual contamination of a pure Chlorine sample with Argon atoms due to the process in Equation 2.6

$$
\begin{aligned}
& N^{0}+\nu \quad \rightarrow \quad P^{+}+\beta^{-} \\
& { }^{37} \mathrm{Cl}+\nu \rightarrow{ }^{37} \mathrm{Ar}+\beta^{-}
\end{aligned}
$$

In 1955, radio-chemist Ray Davis tested this notion with neutrinos from the Brookhaven nuclear reactor [12. He irradiated 1000 gallons of liquid carbon tetrachloride $\left(\mathrm{CCl}_{4}\right)$, 

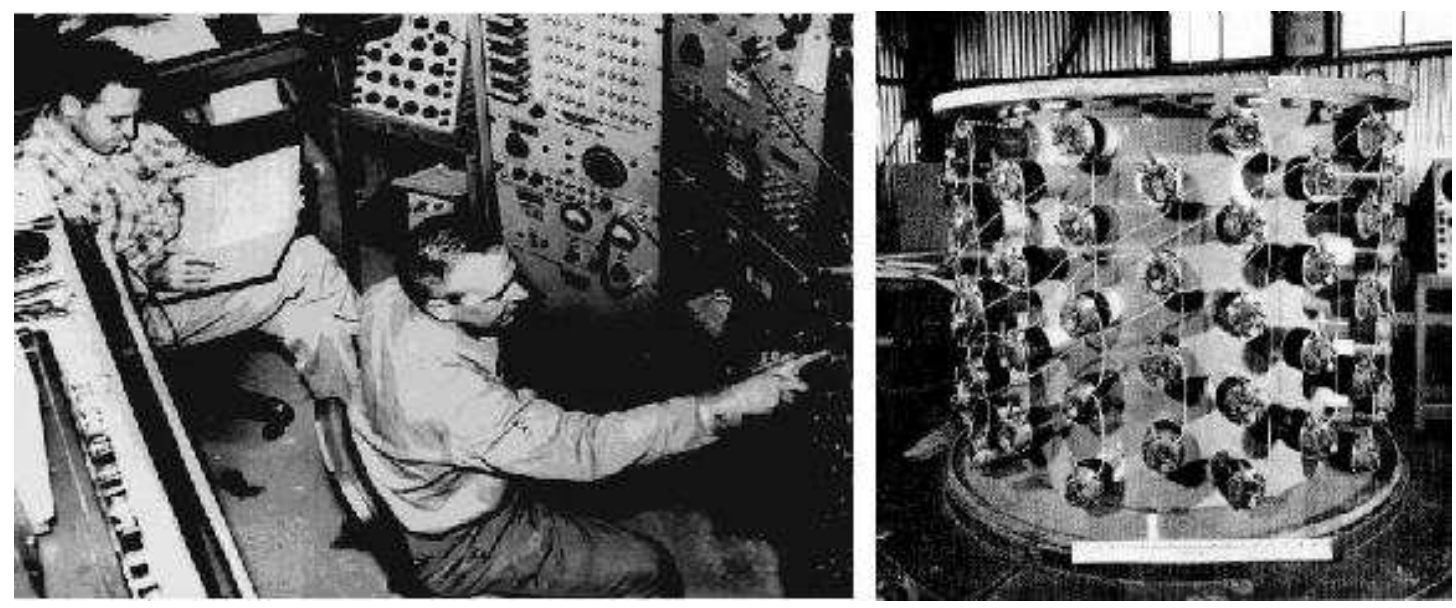

Figure 2.1: Cowan and Reines working at Project Poltergeist, the project that made the first experimental neutrino detection.

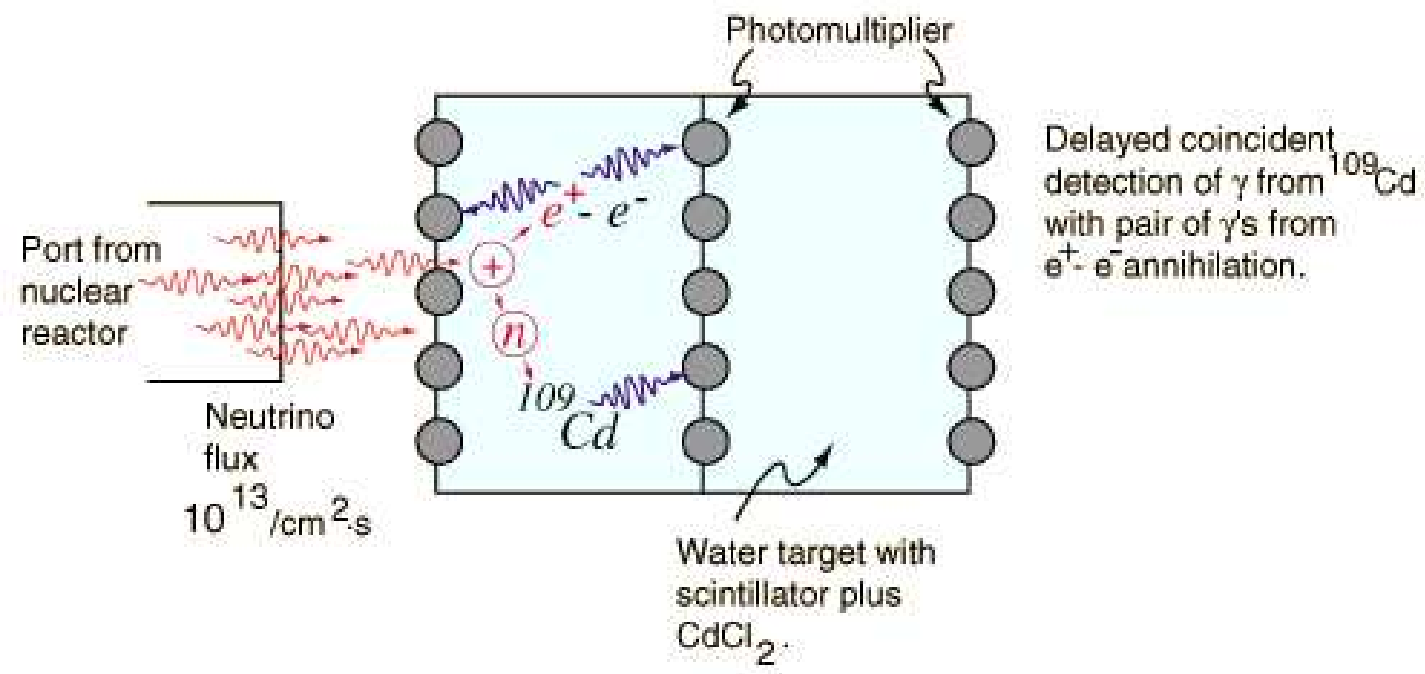

Figure 2.2: Diagram of the Magic Eye neutrino detector [10]. An anti-neutrino would interact with a proton in the water, producing a neutron and positron. The positron annihilates immediately, and the neutron is captured $\sim 10 \mu \mathrm{sec}$ later to produce a delayed two hit signal unique to an anti-neutrino interaction. 
which he extracted Argon atoms from by bubbling helium gas through the fluid. The helium was passed through a liquid-nitrogen cooled charcoal trap that captured the argon atoms. The charcoal filter was then placed in an array of shielded proportional tubes, where the radioactive decay of the unstable argon isotope could be measured by observing the $\beta$ decay process in Equation [2.7] which has a half-life of 35 days.

$$
{ }^{37} A r \quad \rightarrow \quad{ }^{37} A r+\bar{\nu}_{e}+\beta^{-}
$$

He found that the rate of Ar creation was not increasing when he went near the reactor. This meant that the anti-neutrino emitted from the reactor could not cause the process in Equation 2.6] and that $\nu_{e} \neq \bar{\nu}_{e}$. This radiochemical neutrino experiment had a background rate of $\mathrm{Cl} \rightarrow \mathrm{Ar}$ conversions ascribed to cosmic radiation and solar neutrinos. Measurement of the solar neutrino flux by this manner would be later used as a probe into the nuclear processes in sun, and help to prove that neutrinos are massive (Section 2.4.1).

\subsubsection{Measuring the Neutrino Mass}

In his 1934 paper, Enrico Fermi asserted that the neutrino mass would be very small compared to the electron, but that the mass need not necessarily be zero. He predicted that a non-zero neutrino mass would distort the $\beta$-decay spectrum. Energy conservation in the $\beta$-decay process includes Einstein's mass-energy equivalent of $\mathrm{E}=\mathrm{m} \mathrm{c}^{2}$. The maximum energy that the $\beta$ particle in this process can attain is limited by the energy transition of the decay products, and the mass of the neutrino.

The sensitivity of electron spectroscopy was steadily improved in the early $20^{\text {th }}$ century. The $\beta$ decay of tritium $\left({ }^{3} H \rightarrow{ }^{3} H e+\beta^{+}\left(E_{\beta}\right)+\bar{\nu}\left(E_{\bar{\nu}}\right)\right)$ provided a good potential for examining this possibility. Most other radioactive isotopes that experience $\beta$-decay have a larger energy transition, and a small difference in the end point of the $\beta$ spectrum would be more difficult to measure. Tritium $\beta$ decay has the lowest energy transition of the radioactive elements, therefore the $\beta$ spectrum from this decay offered the best chance for observing distortion [13].

In 1952, Langer and Moffat [14] tested the tritium $\beta$-decay spectrum with a magnetic spectrometer. They found that the end point of the $\beta$ spectrum was not distorted with 
in the precision of their experiment, and that the mass of the $\nu_{e}$ could be no more than $250 \mathrm{eV}$.

\subsubsection{Neutrino Flavor}

Cosmic-ray physicists discovered the $\mu$ and $\pi$ particles in the 1940s, and with that found another method of neutrino production. The decay of $\pi$ particles was observed in photographic film to a $\mu$ with a kinked path [15]. The kink in the path at the decay point was determined to be the result of an accompanying neutrino in the decay. During this time, the newly discovered $\mu$ particle was identified as a heavier version of the $\beta$. Some

physicists of the time decided that the neutrinos emitted in the two different processes (nuclear $\beta$ decay and $\pi$ to $\mu$ decay) would have to be different types of neutrinos.

Pontecorvo suggested that if the neutrinos from the pion decays were different from those in the nuclear $\beta$ decays, then interactions of these neutrinos should be different. The neutrino interaction process in equation 2.8 had been observed for neutrinos that are emitted during beta decay, and the process in equation 2.9 had been observed for the type of neutrinos emitted during pion decay.

$$
\begin{aligned}
& n^{+}+\stackrel{(-)}{\nu} \rightarrow \beta^{-}+n^{+} \\
& n^{+}+\stackrel{(-)}{\nu} \rightarrow \mu^{-}+n^{+}
\end{aligned}
$$

A large number of $\pi$ particles would be required to examine the neutrinos produced by $\pi$ decays. The $\pi$ s would decay after a time, and produce a stream of neutrinos. It was suggested in 1960 [16, 17, 16] that a high energy proton beam could strike a target to produce a source of pions. A neutrino detector would be situated a sufficient distance from the target allow the neutrinos to decay, and surrounded in heavy shielding. This premise for creating a neutrino beam is still in use for modern neutrino beams.

In 1962 a group of physicist at Brookhaven National Laboratory used the Alternating Gradient Synchrotron (AGS) proton beam to create a beam of neutrinos that could test this theory [18. The $15 \mathrm{GeV}$ proton beam was diverted from its circular path and pointed at a Beryllium target. A spark chamber with 10 tons of aluminum served as a neutrino detector, was situated $21 \mathrm{~m}$ away, shielded by steel and concrete. If a neutrino interacted in the detector via Equation [2.9, the resulting muon would produce 


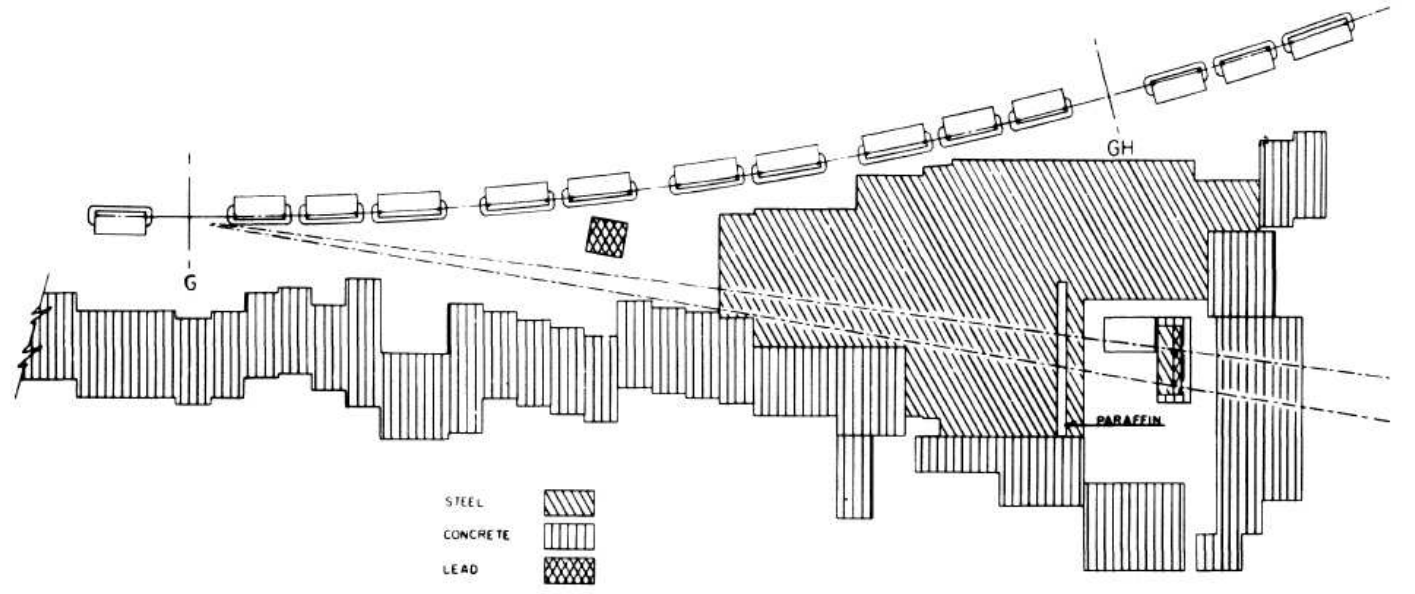

Figure 2.3: The AGS neutrino experiment created a primitive $\nu_{\mu}$ beam with $\pi$ particle decay, and observed the neutrinos in a shielded spark chamber [18].

a contained track-like event in the spark chamber. If a neutrino interacted via Equation 2.8 the resulting electron would produce a contained showering event in the spark chamber.

The experiment detected 29 track-like events and 6 showering events. With Poisson counting statistics, the likelihood of the same number fluctuating to both 29 and 6 is $\sim 0.4 \%$. The few showering events were expected to come from either muon misidentification or $K^{+}$decays. The AGS neutrino experiment verified that there are two types of neutrinos $\left(\nu_{\mu} \neq \nu_{e}\right)$ by showing that neutrinos from pion decay do not react by the process in Equation 2.8. This result was a starting point for the notion of particle generations or flavors.

The Large Electron Positron (LEP) collider at European Organization for Nuclear Research (CERN from the French ConseilEuropenpourlaRechercheNuclaire) started running 1989. There were four detectors at $e+\leftrightarrow e^{-}$collision points, Aleph, Delphi, Opal, and L3. Electron-positron colliders have the benefit of fine energy tuning, as the two beams can have their energies fine tuned to produced a collision with a known center of mass energy. The four detectors combined to measure the cross section for the $\mathrm{Z}^{0}$ boson to decay into hadrons at various center of mass energies. This measurement found that there could only be 3 lepton generations [19]. 


\subsection{Interaction of Neutrinos with Matter}

With Project Poltergeist, Cowan and Reines proved Fermi's conjecture that the interaction of a neutrino with matter would be an extremely rare instance. Their experiment, along with all future neutrino experiments, have relied on an intense flux of neutrinos and massive target to detect neutrino interactions. A neutrino has never been, and may never be directly observed due to the extremely low interactivity between neutrinos and matter. The detection of neutrino is performed indirectly by observing the resulting particles from the interaction of a neutrino with normal matter (proton, neutron, or electron). This section briefly outlines the theory behind the various forms of neutrino interaction with matter, and how this pertains to the detection of neutrinos.

The current standard model of particle physics dictates that neutrinos do not participate in electromagnetic or nuclear-strong interactions, but only in electroweak interactions. The theory of electroweak interactions was birthed by Fermi in the description of the beta decay. His theory was later developed into the GlashowWeinbergSalam $(G W S)$ model of vector-axial (VA) currents. The GWS model predicts two charged boson $\left(W^{ \pm}\right)$and a neutral boson $\left(Z^{0}\right)$. The exchange of an electroweak bosons will conserve electrical charge and lepton flavor. For more in depth information on the theory of VA currents, see [20, 21].

The neutrino interaction process of interest in the MINOS far detector is the scattering of a neutrino off of a nucleon. If the scattering interaction is the result of a neutral $\mathrm{Z}$ boson exchange, the process is a neutral-current (NC) interaction (Figure 2.4). The hadronic system that results from a NC interaction doesn't offer a full representation of the energy of the incident neutrino. The energy carried by the Z-boson in the scattering process, combined with the energy of the target, is equivalent to the energy of the hadronic system. Neglecting the target energy, the energy of the hadronic system is called the "visible neutrino energy", which is the energy lost by the neutrino in the scattering process. Energy reconstruction of a NC interaction can only be used to set an upper limit on the neutrino energy.

A neutrino-nucleon scattering interaction that is mediated by a charged electroweak boson is a charged-current (CC) interaction (Figure [2.5). CC interactions result in a lepton with the same flavor as the incident neutrino, and a hadronic system. The visible 


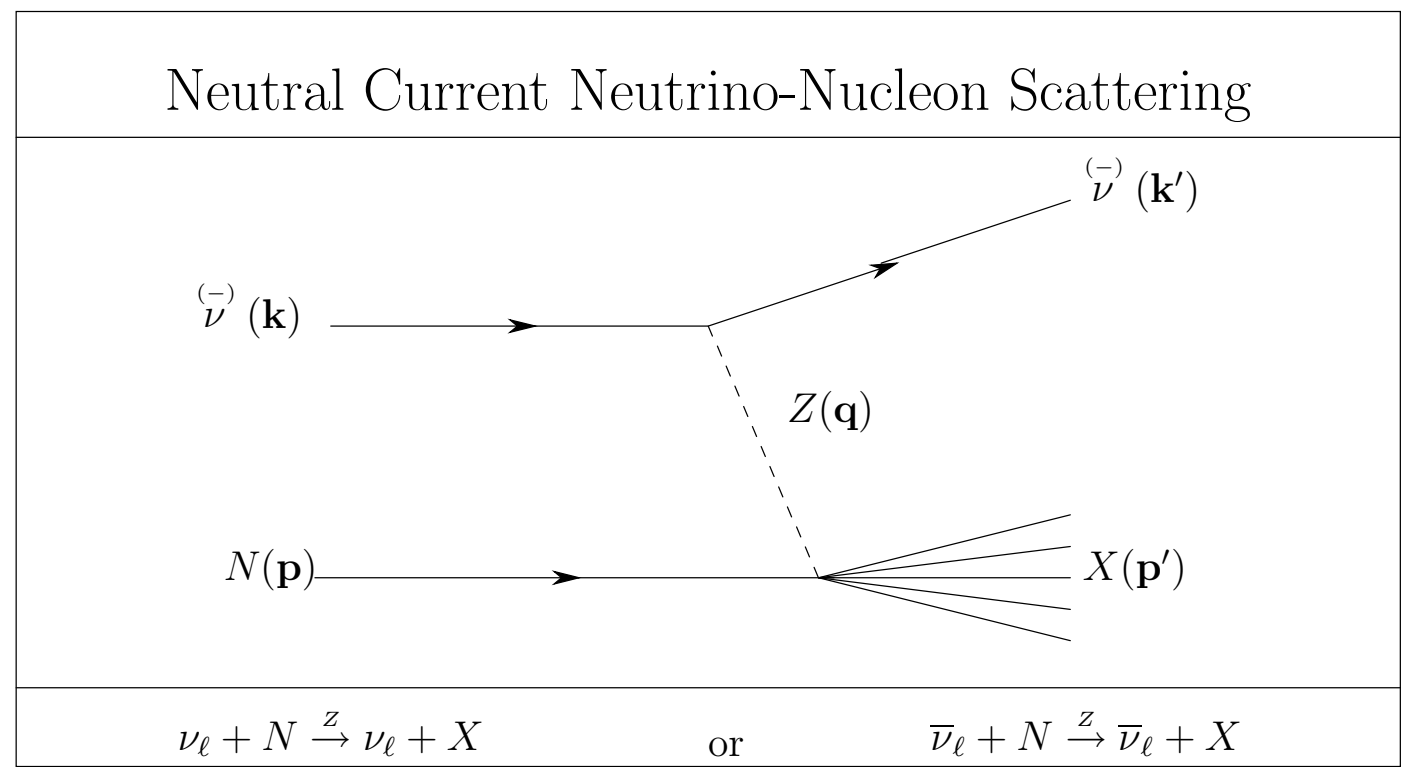

Figure 2.4: The structure of an incoming neutrino with 4-momentum $\mathbf{k}$, exchanging a $\mathrm{Z}$ boson $(\mathbf{q})$ with a nucleon $(\mathbf{q})$, resulting in the neutrino $\left(\mathbf{k}^{\prime}\right)$ and a hadronic system $\mathrm{X}$ $\left(\mathbf{q}^{\prime}\right)$

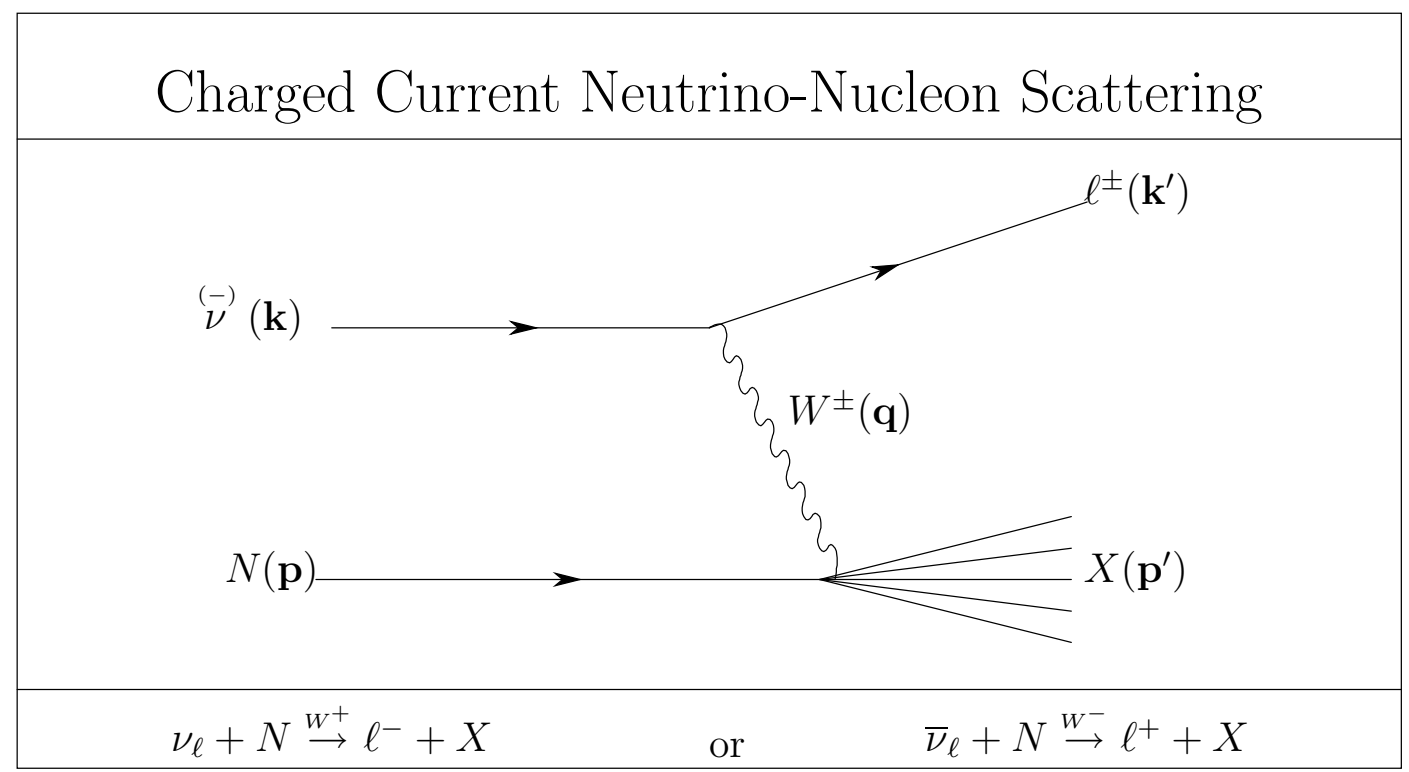

Figure 2.5: The structure of an incoming neutrino with 4-momentum $\mathbf{k}$, exchanging a $\mathrm{W}$ boson $(\mathbf{q})$ with a nucleon $(\mathbf{q})$, resulting in a lepton $\left(\mathbf{k}^{\prime}\right)$ and hadronic system $\mathrm{X}\left(\mathbf{q}^{\prime}\right)$ 
neutrino energy for a CC interaction is the sum of the energy carried by the charged lepton and the energy of the hadronic system. The entire energy of the neutrino is lost this type of interaction, and thus the entire neutrino energy can be reconstructed from the lepton and hadronic system energy.

The hadronic systems shown in Figures 2.4 and 2.5 appear as a spray of particles. The hadronic system that results from the electroweak boson exchange with a nucleon will appear as spray of particles if the nucleon is itself broken apart. In the process of deep inelastic scattering (DIS), the neutrino exchanges a boson with a quark-parton function inside of the nucleon. If the nucleon is not broken apart by the exchange of the boson, the process is referred to as quasi-elastic (QE) scattering. A QE interaction is distinguished from a DIS interaction by a nucleon ejected from its nucleus rather than a spray of pions and other light hadrons.

The interaction of a neutrino with a nucleon is an important process to understand for the analysis presented in this thesis. When a neutrino interacts via the electroweak force, it does so as a pure lepton flavor. Recent developments in neutrino physics have suggested that if neutrino have mass the neutrino flavor state may not be identical to the neutrino mass state. The consequence of this is that neutrino flavor may change as a neutrino travels, a phenomenon known "neutrino oscillation". The next section examines the theory and phenomenology of neutrino oscillation.

\section{$2.3 \quad$ Neutrino Oscillation}

If the neutrino has a non-zero mass, the quantum state of the neutrino can be described in two different manners, as a lepton flavor state $\left(\left|\nu_{\alpha}\right\rangle\right)$ or as a massive state $\left(\left|\nu_{k}\right\rangle\right)$. A neutrino expressed in lepton flavor state couples to the electroweak Lagrangian and may interact with matter as described in Section 2.2. A neutrino expressed as a massive state is an eigenstate of the Hamiltonian and a solution to Schrödinger's Equation. Even though there are as many flavored states as massive states, each flavored states does not necessarily correspond to a single massive state. Each flavored neutrino state is instead expressed as a superposition of all massive neutrino states with an appropriate mixing matrix $\mathrm{U}$ that is unitary $\left(\mathrm{U}^{\dagger} \mathrm{U}=1\right.$ and $\left.\mathrm{UU}^{\dagger}=1\right)$ as in Equation 2.10. This mixing matrix is often referred to as the Pontecorvo-Maki-Nakagawa-Sakata (PNMS) matrix 
$22,23]$.

$$
\left|\nu_{\alpha}\right\rangle=\sum U_{\alpha k}\left|\nu_{k}\right\rangle
$$

Since the massive neutrino states are eigenstates of the Hamiltonian, in a vacuum they will evolve over time as shown in Equation 2.11 where $\tau$ is the proper time in the neutrino rest frame and $m_{k}$ is the $\mathrm{k}^{\text {th }}$ neutrino mass. If the $\mathrm{k}^{\text {th }}$ massive neutrino is boosted to a lab rest frame where the massive neutrino has a four momentum $\left(\mathbf{p}_{k}=\right.$ $\left.\left[E_{k}, \vec{p}_{k}\right]\right)$ and will traverse a four vector distance $(\mathbf{d}=[t, \vec{d}])$, then the exponential term becomes $m_{k} \tau=\mathbf{p}_{k} \cdot \mathbf{d}=E_{k} t-\vec{p}_{k} \cdot \vec{d}$. A neutrino will propagate along the direction of its momentum, which will make the dot product of the momentum and propagation vectors be the product of the magnitude of both vectors $\left(\vec{p}_{k} \cdot \vec{d} \rightarrow|\vec{p}| L\right.$, where $\mathrm{L}$ is the distance traveled in the lab frame). When the time evolution in Equation 2.11 is boosted to the lab frame, it changes to Equation 2.12 .

$$
\begin{aligned}
\left|\nu_{k}(\tau)\right\rangle & =e^{-i m_{k} \tau}\left|\nu_{k}(0)\right\rangle \\
\left|\nu_{k}(\mathbf{p}, \mathbf{d})\right\rangle & =e^{-i(E t-|\vec{p}| L)}\left|\nu_{k}(0)\right\rangle
\end{aligned}
$$

Neutrino detection relies on a high neutrino energy for two reasons. First, the cross section of neutrino-nucleon interaction is directly proportional to the neutrino energy. Second, detecting a neutrino interaction relies on observing the lepton emitted in the process, and modern lepton detection technology set a lower limit on the energy of detectable leptons. It is therefore reasonable to assume that the energy of a neutrino detected in an oscillation experiment is much larger than the neutrino mass. Given this, an approximate relationship can be drawn between the neutrino's energy and momentum. The relativistic relationship between energy, momentum and mass is $p^{2}=$ $E^{2}-m^{2}$ or $|p|=\sqrt{E^{2}-m^{2}}$. The right hand side of this equation can be expanded and expressed as $p=E-\frac{m^{2}}{2 E}$ for $m<<E$. By substituting the momentum in Equation 2.12 with the expanded from, and assuming the $t \sim L$ for a relativistic neutrino, the time evolution of the massive neutrino is expressed in equation 2.13

$$
\left|\nu_{k}\left(E_{k}, L\right)\right\rangle=e^{-i \frac{m_{k}{ }^{2} L}{2 E_{k}}}\left|\nu_{k}(0)\right\rangle
$$


The time evolution in Equation 2.13 is for a massive neutrino state, but any neutrino at the moment it is created via an electroweak decay is in a flavored neutrino state. The superposition of massive neutrino states in Equation 2.10 can be combined with the time evolution of massive states in Equation 2.13 to arrive at the time evolution of flavored states in Equation 2.14. The different massive neutrino states in Equation 2.14] have been reduced to a single neutrino energy $\left(E=E_{j}\right.$ for all $\left.\mathrm{j}\right)$. This assumption is somewhat limiting, and is handled more rigorously with a full wave analysis [24].

$$
\left|\nu_{\alpha}(E, L)\right\rangle=\sum_{k} U_{\alpha k} e^{-i \frac{m_{k}{ }^{2} L}{2 E}}\left|\nu_{k}(0)\right\rangle
$$

After traveling some distance and time, the quantum probability fields of each massive neutrino state will develop slightly different magnitudes due to the different $m_{k}{ }^{2}$ factors in each massive neutrino state propagator. When that neutrino interacts with matter after traveling some distance from its point of creation, its quantum field is collapsed and expressed as a single lepton flavor. Neutrino oscillation is defined as the probability that the lepton flavor the neutrino eventually interacts as is different from the flavor it was created as.

A simple analogy is drawn to neutrino oscillation by replacing a neutrino with a collection of different sized coins, where each coin represents a massive neutrino. The analogy is completed by representing the flavored neutrino state as the number of coins that are showing matching sides. If there are two massive and flavored neutrinos, a neutrino can be represented by a nickel and a dime. If the two coins are showing opposite faces (heads and tails) the neutrino is of type $\alpha$. If the two coins are showing matching faces (heads and heads or tails and tails) then the neutrino is of type $\beta$. The nickel and dime are set on a spring board with an initial state of both heads up (in state $\beta$ ), and the two coins are flung together in the same direction with the same angular momentum. Because the two have different masses, the same angular momentum will cause the two coins to rotate at different rates. When the coins land, each will have rotated a different amount, and the pair may land as either matching faces (state $\beta$ ) or opposite faces (state $\alpha$ ). Until the coins land they are continually rotating, and are in neither of the possible states until they hit the ground and the faces are forced into a state. Due to the rotating nature of the coins, the probability of landing as a 
given state depends periodically on both magnitude of the angular momentum and the distance that the coins have traveled.

Drawing back to actual neutrinos, the probability that the collapsed waveform of the flavored neutrino state $\left|\nu_{\alpha}(E, L)\right\rangle$ in Equation 2.14 will express itself as another flavored neutrino state $\left(\left|\nu_{\beta}\right\rangle\right)$ is measured by taking the magnitude of the inner product between the final neutrino flavor state and time-evolved neutrino flavor state, as expressed in Equation 2.15. This inner product is found in terms of neutrino energy and propagation length by combining Equations 2.10 and 2.14 to arrive at Equation 2.16.

The inner product terms can be expanded, and the general form of neutrino oscillation probability is expressed in Equation 2.17 if the initial and final neutrino state differ, where $\Delta m^{2}{ }_{k j}=m_{k}{ }^{2}-m_{j}{ }^{2}$. Because of the symmetry of the $U_{\beta k}^{\star} U_{\alpha k} U_{\beta j} U_{\alpha j}^{\star}$ factor, the probability of $\nu_{\alpha}$ oscillating to $\nu_{\beta}$ is identical to the probability of the inverse $\left(P\left(\nu_{\alpha} \rightarrow \nu_{\beta}\right)=P\left(\nu_{\beta} \rightarrow \nu_{\alpha}\right)\right)$.

The probability that the flavored neutrino state will be the same at the point of interaction as it was as the point of creation is expressed in Equation 2.18. This probability is different from the form expressed in Equation 2.17 in that any $\mathrm{CP}$ violating imaginary phases in the mixing matrix will not affect the oscillation.

$$
\begin{aligned}
P\left(\nu_{\alpha} \rightarrow \nu_{\beta} ; E, L\right)= & \left|\left\langle\nu_{\beta} \mid \nu_{\alpha}(E, L)\right\rangle\right|^{2} \\
= & \left|\sum_{k} U_{\alpha k} U_{\beta k}^{\star} e^{-i m_{k}{ }^{2} \frac{L}{2 E}}\right|^{2} \\
P\left(\nu_{\alpha} \rightarrow \nu_{\beta \neq \alpha} ; E, L\right)= & 2 \sum_{k>j} \operatorname{Im}\left(U_{\beta k}^{\star} U_{\alpha k} U_{\beta j} U_{\alpha j}^{\star}\right) \sin \left(\frac{L \Delta m^{2} k j}{2 E}\right) \\
& -4 \sum_{k>j} R e\left(U_{\beta k}^{\star} U_{\alpha k} U_{\beta j} U_{\alpha j}^{\star}\right) \sin ^{2}\left(\frac{L \Delta m^{2}{ }_{k j}}{4 E}\right) \\
P\left(\nu_{\alpha} \rightarrow \nu_{\alpha} ; E, L\right)= & 1-4 \sum_{k>j}\left(\left|U_{\alpha k}\right|^{2}\left|U_{\alpha j}\right|^{2}\right) \sin ^{2}\left(\frac{L \Delta m_{k j}^{2}}{4 E}\right)
\end{aligned}
$$

The sinusoidal terms in Equations 2.17 have a periodic dependence on $\frac{L \Delta m^{2}}{E} \Delta m^{2}$. Given that $\Delta m^{2}$ is a constant value, the probability of a neutrino flavor change depends periodically on the initial neutrino energy and the distance traveled, just as was the case in the coin analogy. The dependence on the $\Delta m^{2}$ in Equations 2.17 and 2.18 
demonstrates that if neutrinos are either not massive or have identical masses, neutrinos will not oscillate between flavors. To further develop the practical use of neutrino oscillation phenomenology, the formalism must be specialized for a specific number of flavored and massive neutrinos. This is first dealt with in the simplest possible case of two neutrino oscillation, and then in the slightly more complex case of three neutrino oscillation.

\subsubsection{Two Neutrino Oscillation}

If there are only two flavored and massive-neutrino states, the unitary mixing matrix $(U)$ can be expressed with a single mixing angle $(\theta)$ and an imaginary phase $(\delta)$. For this case, the general neutrino mixing formulation in Equation 2.10] is simplified to the formulation in Equation 2.19

$$
\left(\begin{array}{c}
\nu_{\alpha} \\
\nu_{\beta}
\end{array}\right)=\left(\begin{array}{cc}
\cos \theta & \sin \theta e^{i \delta} \\
-\sin \theta e^{-i \delta} & \cos \theta
\end{array}\right)\left(\begin{array}{c}
\nu_{1} \\
\nu_{2}
\end{array}\right)
$$

If only two neutrino types are considered, the oscillation probability from Equations 2.17 and 2.18 can only take two forms: the initial neutrino oscillates to the other neutrino flavor $\left(P\left(\nu_{\alpha} \rightarrow \nu_{\beta}\right)\right.$, as in Equation [2.20) , or the neutrino oscillates to it's original flavor $\left(P\left(\nu_{\alpha} \rightarrow \nu_{\alpha}\right)\right.$, as in Equation 2.21). In both cases the oscillation probability has lost the imaginary term in the unitary matrix.

$$
\begin{aligned}
& P\left(\nu_{\alpha} \rightarrow \nu_{\beta}\right)=\sin ^{2}(2 \theta) \sin ^{2}\left(\frac{L \Delta m^{2}}{4 E}\right) \\
& P\left(\nu_{\alpha} \rightarrow \nu_{\alpha}\right)=1-\sin ^{2}(2 \theta) \sin ^{2}\left(\frac{L \Delta m^{2}}{4 E}\right)
\end{aligned}
$$

The probability that a neutrino will oscillate away from its initial flavor $\left(1.0-P\left(\nu_{\alpha} \rightarrow\right.\right.$ $\left.\nu_{\alpha}\right)$ ) is often referred to as the "oscillation disappearance probability". Experiments that seek to explain anomalous deficits in neutrino interaction rates with neutrino oscillation are called disappearance oscillation experiments. These types of experiments are discussed in Section 2.4.1 regarding the solar neutrino anomaly and Section 2.4.2 regarding the atmospheric neutrino anomaly. 
The probability that a neutrino will oscillate to another specific flavor $\left(P\left(\nu_{\alpha} \rightarrow \nu_{\beta}\right)\right)$ is often referred to as the "oscillation appearance probability". Experiments that search for anomalously high neutrino rates in an unexpected neutrino flavor are called appearance oscillation experiments. When two neutrino flavors are used to devise the neutrino oscillation formulation, the appearance probability and disappearance probabilities will be equivalent. The next section presents three neutrino oscillation formulation, where this is not necessarily the case.

\subsubsection{Three Neutrino Oscillation}

When three neutrinos are considered instead of two, the unitary matrix can no longer be expressed with a single mixing angle. There will now be three angles to describe the mixing between each of the three massive neutrino states $\left(\theta_{12}, \theta_{23}, \theta_{31}\right)$, accompanied by a single imaginary phase angle $(\delta)$. To simplify the statement of the new mixing matrix, the angles are also expressed as $s_{12}=\sin \left(\theta_{12}\right), c_{12}=\cos \left(\theta_{12}\right)$, etc. Each mixing angle contributes a mixing matrix, analogous to the mixing matrix in Equation 2.19 The full unitary mixing matrix is the product of the three mixing matrices $\left(U_{12}, U_{23}\right.$, and $\left.U_{31}\right)$, as shown in equation 2.22. The fourth mixing matrix $\left(U_{\text {Majorana }}\right)$, contains a set of phases that are only relevant for Majorana neutrinos, that is if massive neutrino state is identical to its antiparticle $\left(\nu_{k} \stackrel{?}{=} \bar{\nu}_{k}\right)$. The Majorana phases do not affect oscillation, and are disregarded in further formulation [19].

$$
\begin{gathered}
\frac{U_{12}}{\Downarrow} \\
U=\left(\begin{array}{ccc}
c_{12} & s_{12} & 0 \\
-s_{12} & c_{12} & 0 \\
0 & 0 & 1
\end{array}\right)\left(\begin{array}{ccc}
1 & 0 & 0 \\
0 & c_{23} & s_{23} \\
0 & -s_{23} & c_{23}
\end{array}\right)\left(\begin{array}{ccc}
c_{31} & 0 & s_{31} e^{-i \delta} \\
0 & 1 & 0 \\
-s_{31} e^{i \delta} & 0 & c_{31}
\end{array}\right)\left(\begin{array}{ccc}
e^{i \alpha} & 0 & 0 \\
0 & e_{i \beta} & 0 \\
0 & 0 & 0
\end{array}\right)
\end{gathered}
$$

If the three neutrino types are labeled electron $\left(\nu_{e}\right)$, muon $\left(\nu_{\mu}\right)$, and tau $\left(\nu_{\tau}\right)$ neutrinos, and the three relevant mixing matrices in Equation 2.22 are combined, the generic mixing from Equation 2.10 is specialized to three neutrino mixing in Equation 2.23. The complex phase angle $\delta$ is called the Dirac phase, and is responsible for $C P$ violation in the lepton mixing sector. 


$$
\left(\begin{array}{c}
\nu_{e} \\
\nu_{\mu} \\
\nu_{\tau}
\end{array}\right)=\left(\begin{array}{ccc}
c_{12} c_{31} & s_{12} c_{31} & s_{31} e^{-i \delta} \\
-s_{12} c_{23}-c_{12} s_{23} s_{31} e^{i \delta} & c_{12} c_{23}-s_{12} s_{23} s_{31} e^{i \delta} & s_{23} c_{31} \\
s_{23} s_{31}-c_{12} c_{23} s_{31} e^{i \delta} & -c_{12} s_{23}-s_{12} c_{23} s_{31} e^{i \delta} & c_{23} c_{31}
\end{array}\right)\left(\begin{array}{c}
\nu_{1} \\
\nu_{2} \\
\nu_{3}
\end{array}\right)
$$

If there are three massive neutrinos, there will only be two unique mass differences $\left(\Delta m^{2}{ }_{31}=\Delta m^{2}{ }_{23}+\Delta m^{2}{ }_{12}\right)$. Experimental evidence for neutrino oscillation, which will be discussed in Section 2.4 has suggested that the two unique $\Delta m^{2}$ scales are largely different $\left(\Delta m^{2}{ }_{23} / \Delta m^{2}{ }_{12} \sim 100\right)$. The two unique $\Delta m^{2}$ values are labeled as the small scale value $\Delta m^{2}{ }_{S}=\Delta m^{2}{ }_{21}$ and the large scale value $\Delta m^{2}{ }_{L}=\Delta m^{2}{ }_{23} \simeq \Delta m^{2}{ }_{31}$. The three-neutrino oscillation probability with this simplification in the $\Delta m^{2}$ scheme is written in Equation 2.24 for neutrino appearance probability, and in Equation 2.25 for neutrino disappearance.

$$
\begin{aligned}
P\left(\nu_{\alpha} \rightarrow \nu_{\beta \neq \alpha}\right)= & 4\left|U_{\alpha 3}\right|^{2}\left|U_{\beta 3}\right|^{2} \sin ^{2}\left(\frac{L \Delta m^{2} L}{4 E}\right) \\
& -4 \operatorname{Re}\left(U_{\beta 1}^{\star} U_{\alpha 1} U_{\beta 2} U_{\alpha 2}^{\star}\right) \sin ^{2}\left(\frac{L \Delta m^{2} S}{4 E}\right) \\
& +2 \operatorname{Im}\left(U_{\beta 1}^{\star} U_{\alpha 1} U_{\beta 2} U_{\alpha 2}^{\star}\right) \sin \left(\frac{L \Delta m^{2} S}{2 E}\right) \\
P\left(\nu_{\alpha} \rightarrow \nu_{\alpha}\right)= & 1-4\left|U_{\alpha 3}\right|^{2}\left(1-\left|U_{\alpha 3}\right|^{2}\right) \sin ^{2}\left(\frac{L \Delta m^{2} L}{4 E}\right) \\
& -4\left|U_{\alpha 1}\right|^{2}\left|U_{\alpha 2}\right|^{2} \sin ^{2}\left(\frac{L \Delta m^{2} S}{4 E}\right)
\end{aligned}
$$

Neutrino oscillation experiments have historically relied on spectral deficits in electron and muon neutrinos to measure oscillation parameters. Tau neutrinos are still too difficult to detect to be able observe any spectral oscillation deficit. The disappearance probability form in Equation 2.25 has been specified for the case of electron-neutrino disappearance in Equation 2.26. This equation presents a dependence on both the $\Delta m^{2}$ scale values. The probability of muon-neutrino disappearance in the case of threeneutrino oscillation is shown in Equation 2.27). Much like the $P\left(\nu_{e} \rightarrow \nu_{e}\right)$ probability, $P\left(\nu_{\mu} \rightarrow \nu_{\mu}\right)$ also depends on both of the $\Delta m^{2}$ scales. The coefficient has not been expanded in front of the $\sin ^{2}\left(\frac{L \Delta m^{2} S}{4 E}\right)$ in terms of mixing angle. The muon neutrinos 
available to inspect for spectral deficits (atmospheric and beam) are not in a range of $\frac{L}{E}$ values that is sensitive to $\frac{L \Delta m^{2} S}{4 E}$, and the $\sin ^{2}\left(\frac{L \Delta m^{2} S}{4 E}\right)$ term in Equation [2.27] is irrelevant.

$$
\begin{aligned}
P\left(\nu_{e} \rightarrow \nu_{e}\right)= & 1-\sin ^{2}\left(2 \theta_{31}\right) \sin ^{2}\left(\frac{L \Delta m^{2} L}{4 E}\right) \\
& -\sin ^{2}\left(2 \theta_{12}\right) \cos ^{4}\left(\theta_{31}\right) \sin ^{2}\left(\frac{L \Delta m^{2} S}{4 E}\right) \\
P\left(\nu_{\mu} \rightarrow \nu_{\mu}\right)= & 1-\left(\sin ^{2}\left(2 \theta_{23}\right) \cos ^{2}\left(\theta_{31}\right)+\sin ^{2}\left(2 \theta_{31}\right) \cos ^{2}\left(\theta_{23}\right)\right) \sin ^{2}\left(\frac{L \Delta m^{2} L}{4 E}\right) \\
& -4\left|U_{\mu 1}\right|^{2}\left|U_{\mu 2}\right|^{2} \sin ^{2}\left(\frac{L \Delta m_{S}^{2}}{4 E}\right)
\end{aligned}
$$

Experiments that have detect neutrinos in range of $\frac{L}{E_{\nu}}$ values that are sensitive to the oscillation sinusoidal term with $\Delta m^{2}{ }_{L}$ are not likely to be sensitive to the sinusoidal term with $\Delta m^{2}{ }_{S}$. If $\frac{L}{E_{\nu}}$ hits such a value that $\sin ^{2}\left(\frac{L \Delta m^{2} L}{4 E}=\frac{\pi}{2}\right)=1.0$, then $\sin ^{2}\left(\frac{L \Delta m^{2} S}{4 E}\right) \sim$ 0.01. Conversely, a neutrino oscillation experiment that is sensitive to the smaller $\Delta m^{2} S$ will have to able to detect neutrinos that have a large $\frac{L}{E}$ value. For this case, the value of $\sin ^{2}\left(\frac{L \Delta m^{2} L}{4 E}\right)$ will average across small changes in L and $\mathrm{E}$ to $\frac{1}{2}$.

Since a neutrino oscillation experiment can not be sensitive to both of the $\Delta m^{2}$ values, the disappearance probabilities in Equations 2.26 and 2.27 are constrained to experiment specific cases. An experiment that measures electron-neutrino disappearance on a scale sensitive to $\Delta m^{2}{ }_{S}$ would use the probability in Equation 2.28, This has been accomplished with solar neutrino experiments that are discussed in Section 2.4.1. If the experiment instead measures electron-neutrino disappearance on a scale sensitive to $\Delta m^{2}{ }_{L}$, the probability in Equation 2.29 would be used. This has been accomplished with $\bar{\nu}_{e} \mathrm{~s}$ from nuclear reactors, as presented in Section 2.4 .3

$$
\begin{aligned}
P\left(\nu_{e} \rightarrow \nu_{e}\right) & \simeq 1-\sin ^{2}\left(2 \theta_{12}\right) \sin ^{2}\left(\frac{L \Delta m^{2} S}{4 E}\right)\left(\Delta m^{2}{ }_{S}, \theta_{12} \text { sensitivity }\right) \\
P\left(\nu_{e} \rightarrow \nu_{e}\right) & \simeq 1-\sin ^{2}\left(2 \theta_{31}\right) \sin ^{2}\left(\frac{L \Delta m^{2} L}{4 E}\right)\left(\Delta m^{2}{ }_{L}, \theta_{31} \text { sensitivity }\right) \\
P\left(\nu_{\mu} \rightarrow \nu_{\mu}\right) & \simeq 1.0-\left(\sin ^{2}\left(2 \theta_{23}\right) c_{31}{ }^{2}+\sin ^{2}\left(2 \theta_{31}\right) c_{23}{ }^{2}\right) \sin ^{2}\left(\frac{L \Delta m^{2} L}{4 E}\right)
\end{aligned}
$$




$$
\simeq 1.0-\sin ^{2}\left(2 \theta_{23}\right) \sin ^{2}\left(\frac{L \Delta m^{2} L}{4 E}\right)\left(\Delta m^{2}{ }_{L}, \theta_{23} \text { sensitivity }\right)
$$

Equation 2.30 shows the probability of muon-neutrino disappearance that is measured at values of $\frac{L}{E}$ sensitive to $\Delta m^{2}{ }_{L}$. Results from reactor neutrino experiments suggest that the mixing angle $\theta_{31}$ is very small. For the probability in Equation 2.30. this would make $\sin ^{2}\left(2 \theta_{31}\right) \sim 0$ and $c_{2} 3^{2} \sim 1$.0. Equation 2.31 follows from the notion that $\theta_{23}>\theta_{31}$. Muon-neutrino disappearance is observed with atmospheric neutrino experiments (Section 2.4.2) and beam neutrino experiments (Section 2.4.4).

In addition to the simplified neutrino-disappearance oscillation probabilities mentioned above, neutrino-appearance oscillation probabilities are also of interest. Muonneutrino oscillation is the best modern candidate to observe the neutrino appearance from oscillation. Current experiments that have measured electron-neutrino oscillation have all used electron neutrinos that are mostly less that $10 \mathrm{MeV}$, which is much less than the muon mass $(102 \mathrm{MeV})$. If the muon neutrinos detected have values $\frac{L}{E}$ that are sensitive to $\Delta m^{2}{ }_{L}$, then the probability that a $\nu_{\mu}$ will oscillate to a $\nu_{e}$ or a $\nu_{\tau}$ are in Equations 2.32 and 2.33 respectively. With out the $\Delta m^{2}{ }_{S}$ term, any dependence on the $\theta_{12}$ mixing angle is absent from either of these oscillation forms. The $\nu_{e}$ appearance probability in Equation 2.32 can be exploited by an oscillation experiment with a muon neutrino beam (discussed in Section 2.4.4).

$$
\begin{aligned}
& P\left(\nu_{\mu} \rightarrow \nu_{e}\right)=\sin ^{2}\left(\theta_{23}\right) \sin ^{2}\left(2 \theta_{31}\right) \sin ^{2}\left(\frac{L \Delta m^{2} L}{4 E}\right) \\
& P\left(\nu_{\mu} \rightarrow \nu_{\tau}\right)=\sin ^{2}\left(2 \theta_{23}\right) \cos ^{4}\left(\theta_{31}\right) \sin ^{2}\left(\frac{L \Delta m^{2} L}{4 E}\right)
\end{aligned}
$$

When a neutrino-oscillation experiment is designed, the values of $\mathrm{L}$ and $E_{\nu}$ for which the detector is optimized control that value of $\Delta m^{2}$ to which that experiment will be sensitive. Several of these experiments and their results are reviewed in the next section.

\subsection{Experimental Status of Neutrino Oscillation}

With the onset of multiple experimental methods for observing neutrinos, unexplained phenomena started developing around a lower than expected number of neutrinos. These anomalies introduced the field of neutrino oscillation, which offered a new probe into 
the mass of the neutrino. The theory and phenomenology of neutrino oscillation was presented in section [2.3. This section presents the experimental status of neutrino oscillation.

\subsubsection{Solar Neutrino Anomaly}

In 1955, Ray Davis exploited the conversion of Chlorine atoms to Argon atoms from neutrino interactions to prove that $\nu_{e} \neq \bar{\nu}_{e}$ [12]. This method of neutrino detection had the beneficial ability to detect much lower energy neutrinos than other detection methods that rely on the observation of the lepton from the neutrino interaction. Davis' next step determined that this neutrino detection method could be used to detect neutrinos from the sun. Solar neutrinos are the most abundant neutrinos on the Earth's surface, but have a low enough energy to make detection even more difficult.

Astronomers and astrophysicists have long been trying to understand the nuclear reactions inside of the sun that make it emit light. The sun's source of power is a chain of nuclear fusion reactions that occur in the solar core. These nuclear reactions generate energy in the form for photons, and also produce an intense flux of electron

neutrino. The primary fusion reaction chain is the "proton-proton chain", in which four hydrogen nuclei (protons) fuse and form a helium nucleus (2 protons and 2 neutrons). This reaction is shown in Equation 2.34,

$$
\begin{gathered}
2 e^{-}+4 p \rightarrow{ }^{4} \mathrm{He}+2 \nu_{e}+26.73 \mathrm{MeV} \\
{ }^{2} \mathrm{H}+p \rightarrow \\
\quad \rightarrow
\end{gathered}
$$

There are many other chains of nuclear fusion that also produce the heat that powers the sun (For detailed information, see [25]). The full chain of reactions is called the Standard Solar Model (SSM). The SSM determines the flux of solar neutrinos emitted by each fusion reaction. Figure 2.6 shows the spectrum of solar neutrinos from the various reactions predicted by the SSM. Most solar neutrinos $(>90 \%)$ are from the primary proton-proton interaction $\left(p+p \rightarrow d+e^{+}+\nu_{e}\right)$ of the $p p$ chain. The neutrinos from this interaction have low energies $(<0.4 \mathrm{MeV})$. There are other nuclear reactions 
that produce higher energy neutrinos as well, such as the decay ${ }^{8} B \rightarrow^{8} B e^{*}+e^{+}+\nu_{e}$, which produces neutrinos with energies up to $14.1 \mathrm{MeV}$.

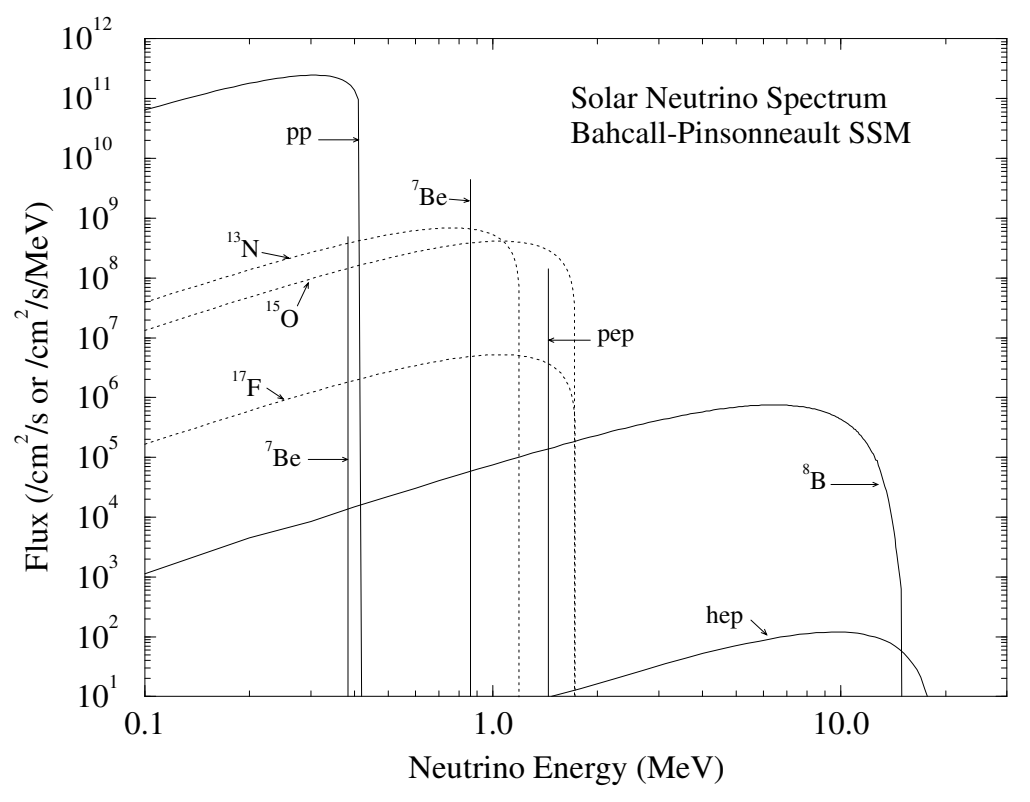

Figure 2.6: Solar neutrino flux spectrum predicted at the Earth's surface [26].

Based on his radiochemical neutrino experiments in the 1950s, Davis conceived an experiment in the early 1960s to measure the flux of neutrino from the sun with inverse beta decay in a deep underground detector [27]. The Homestake radiochemical detector is located in Lead, South Dakota, used 615 tons of $\mathrm{C}_{2} \mathrm{Cl}_{4}$ to detect solar neutrinos by the inverse beta-decay in Chlorine $\left(\nu_{e}+{ }^{37} \mathrm{Cl} \rightarrow{ }^{37} \mathrm{Ar}+e^{-}\right)$. This neutrino interaction process has an energy threshold of $E_{\nu}>814 \mathrm{keV}$, which makes the Homestake detector sensitive to primarily neutrinos from the ${ }^{8} B$ chain. The interaction rate from solar neutrinos in the Homestake detector was expected to be approximately 1 event per day.

The ${ }^{37} \mathrm{Ar}$ was chemically extracted from the tank after two months of accumulation, and was counted with a low background counter. The extraction process of ${ }^{37} \mathrm{Ar}$ from the Homestake perchloro-ethylene tanks had an efficiency $\geq 90 \%$. The Homestake experiment started taking data in 1968, and consistently measured lower rate of $\nu_{e}$ interactions than the prediction made by the SSM. The SSM predicted the interaction rate in the Homestake detector would be $7.6_{-1.1}^{+1.3}$ Solar Neutrino Units (SNU), but the observed rate was $2.56 \pm 0.16 \pm 0.16 \mathrm{SNU}$. The SNU is a measurement devised by John 
Bahcall to measure the rate of neutrino interactions at earth due to solar neutrinos, and is equivalent to 1 interaction per $10^{36}$ target atoms. The deficit of $\nu_{e}$ deficit was referred to as the "solar neutrino anomaly" [28].

Other experiments have also attempted to measured the rate of solar neutrinos, and verify or reject the results from the Homestake mine. In the 1980s the next generation of radiochemical experiments were developed bases on the principle of gallium neutrino capture $\left(\nu_{e}+{ }^{71} G a \rightarrow{ }^{71} G e+e^{-}\right)$. This inverse-beta decay process with a Gallium atom has a threshold energy of $0.23 \mathrm{MeV}$, which, unlike the Chlorine radiochemical experiments, make this type of experiment sensitive to neutrino created in the primary proton-proton reaction. The Soviet American Germanium Experiment (SAGE [29]) and European Gallium Experiment (GALLEX [30]) were developed to this to make this measurement in the Baksan and Gran Sasso underground laboratories respectively. Both SAGE and GALLEX observed an anomalous deficit in solar neutrino, much like the Homestake experiment.

Later in the 1980s, a new method for solar neutrino detection was developed at the Kamiokande experiment. Kamiokande used a deep underground detector (under Mt. Ikenoyama in Japan) that observed the signal of Čerenkov light from high-energy charged particle passing through pure water. Their tank contained $3 \mathrm{kt}$ of ultra-pure water surrounded by 900 PMTs. They were able to detect of neutrinos from the sun due to elastic scattering of neutrinos with electrons $\left(\nu_{e}+e^{-} \rightarrow e^{-}+\nu_{e}\right)$. The recoil electron from this process produces the Cerenkov radiation that is detected by the PMTs. Solar neutrino are identified and distinguished from background radiation by those events that point back to the sun. Only Solar neutrinos above $7 \mathrm{MeV}$ could be positively by Kamiokande, which according to the SSM made them sensitive primarily to ${ }^{8} B$ neutrinos. With the solar neutrino data that Kamiokande observed between 1987 and 1995, a significant deficit in the rate of solar neutrinos was observed, much like the radiochemical experiments [31].

The Kamiokande experiment received an order of magnitude upgrade in 1995 to 50 kt of water and 13,000 PMTs. The new Super-Kamiokande(SKK) consisted of an inner fiducial detector with $20 \mathrm{kt}$ of water surrounded by 11,000 PMTs, and an outer veto detector with $30 \mathrm{kt}$ of water monitored by 2,000 PMTs. The purity of the water in SKK had been improved enough over the water in Kamiokande to reduce the solar neutrino 
neutrino detection threshold down to $5 \mathrm{MeV}$ from $7 \mathrm{MeV}$. SKK has repeated the solar neutrino flux measurement of Kamiokande with higher precision, and found a similar deficit in from the SSM expectation [32].

The solar neutrino anomaly can be explained with neutrino oscillations, specifically the oscillation of $\nu_{e}$ to $\nu_{\mu} / \nu_{\tau}$. The three types of solar neutrino detectors result in a deficit in the measured $\nu_{e}$ flux relative to the SSM expectation. These combined analyses presented three possible neutrino oscillation solutions:

- large mixing angle(LMA) - $\Delta m^{2} \sim 2 \times 10^{-5} e V^{2}, \sin ^{2}(2 \theta) \sim 0.76$

- small mixing angle(SMA) - $\Delta m^{2} \sim 5 \times 10^{-6} e V^{2}, \sin ^{2}(2 \theta) \sim 6 \times 10^{-3}$

- low $\operatorname{mass}(\mathrm{LOW})-\Delta m^{2} \sim 8 \times 10^{-8} e V^{2}, \sin ^{2}(2 \theta) \sim 0.96$

Radiochemical and water Čerenkov solar neutrino detectors are sensitive primarily to the $\nu_{e}$ flux and may have observed the disappearance of $\nu_{e}$ due to oscillation, but have not observed a corresponding appearance of $\nu_{\mu}$ or $\nu_{\tau}$. The definitive detection of a $\nu_{\mu}$ or $\nu_{\tau}$ requires a charged-current interaction, but solar neutrinos fall well below the $\mu$ and $\tau$ masses. The solar neutrino detector in the Sudbury Neutrino Observatory (SNO in Figure 2.7) detects neutral-current interactions from all three neutrino flavors, and uses these to measure oscillation.

SNO is located in Sudbury, Ontario, and contains $1 \mathrm{kt}$ of heavy water $\left(\mathrm{D}_{2} \mathrm{O}\right)$. Three neutrino interactions can be detected in the heavy water: $\nu_{e}$ charged current (CC) $\left(\nu_{e}+d \rightarrow p+p+e^{-}\right), \nu$ neutral current (NC) $\left(\nu_{x}+d \rightarrow n+p+e^{-}\right)$, and $\nu$ elastic scattering (ES) interactions $\left(\nu_{x}+e^{-} \rightarrow e^{-}+\nu_{x}\right)$. ES interactions are sensitive to all three neutrino flavors, but with cross-sections for $\nu_{\mu}$ and $\nu_{\tau}$ ES is $\sim \frac{1}{6}$ of the crosssection for $\nu_{e}$ ES. The rates of each interaction can be combined to measure the solar neutrino flux, by virtue of the varying oscillation sensitivity of each interaction type.

The SNO experiment has been performed in three phases: the first phase started in May 1999, and $\mathrm{NC}$ events are detected with the emission of a $6 \mathrm{MeV}$ gamma ray following neutron capture, the second phase started in May 2001, when 2 tons of high purity table salt $(\mathrm{NaCl})$ was added to the water to increase the neutron capture efficiency and the gamma ray multiplicity, the third phase started in January 2005, when the salt was removed and ${ }^{3} \mathrm{He}$ proportional counters are place in the heavy water to detect the 
SNO Schematic View

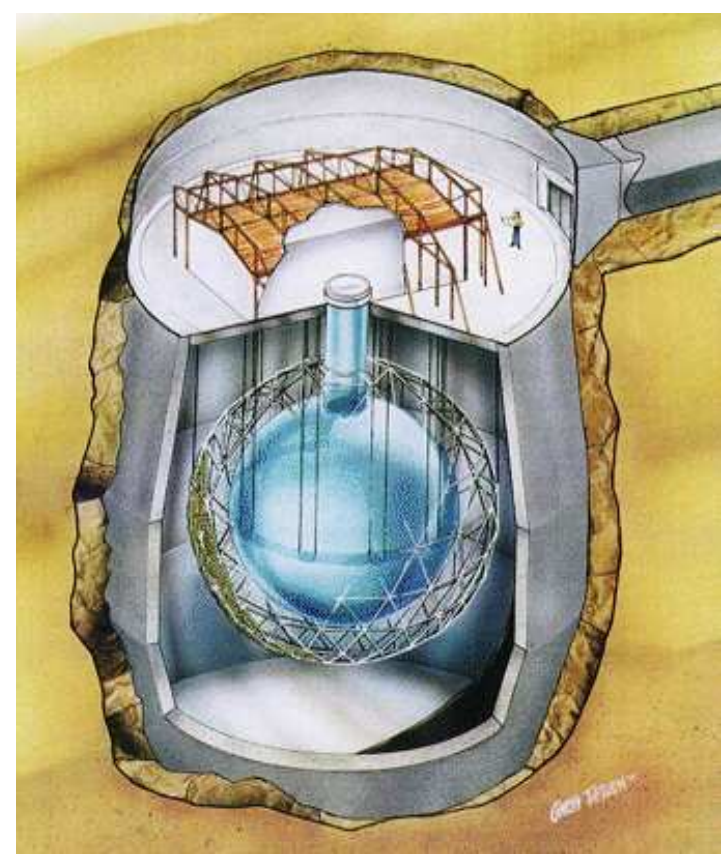

Personel During Installation

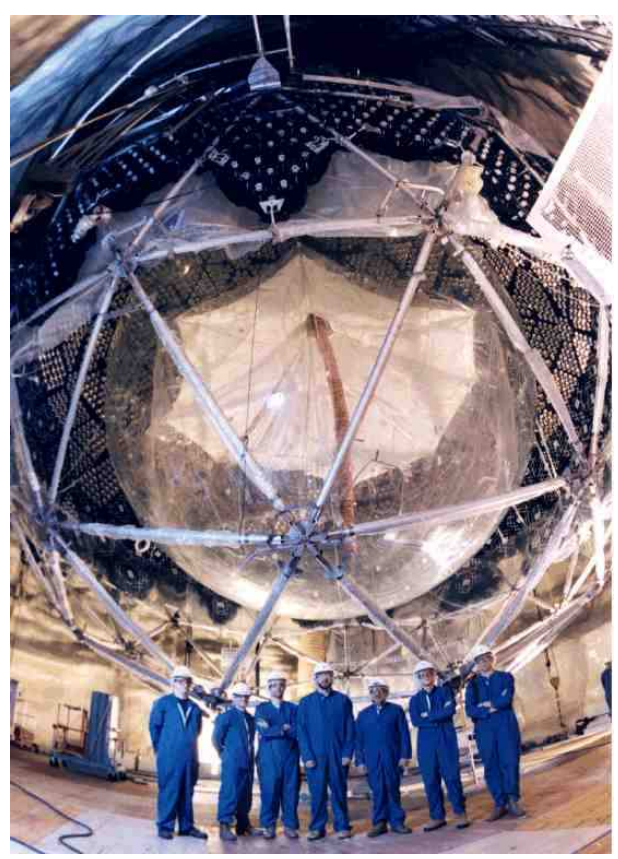

Figure 2.7: The Sudbury Neutrino Observatory (SNO) detects solar neutrinos with 1 kt of heavy water.

neutrons. The results from the salt phase of SNO are shown in Figure 2.8 33. Figure 2.8 a has the non- $\nu_{e}$ component of the flux $\left(\phi_{\mu \tau}\right)$ for CC, NC, and ES type interactions plotted against the same for the $\nu_{e}$ flux component $\left(\phi_{e}\right)$. All the measurements share a a common location in the plot where the non- $\nu_{e}$ flux is non-zero.

The flux measurements in Figure 2.8 a have been used to determine the oscillation parameters $\left(\Delta m^{2}\right.$ and $\left.\theta\right)$ for $\nu_{e} \rightarrow \nu_{\ell \neq e}$ oscillations, shown in Figure 2.8. b. The oscillation analysis for solar neutrino includes the effect of matter on neutrino oscillations, known as the Mikheyev - Smirnov - Wolfenstein (MSW) effect named for the three theorists [34, 35]. These physicists determined that neutrino oscillation is resonantly enhanced in a dense medium (such as in the sun) due to interactions that $\nu_{e}$ experience and $\nu_{\mu / \tau}$ do not experience. All three neutrino flavors experience NC interactions, but $\nu_{e}$ undergo additional ES interactions with the densely packed electrons in the solar core. This modifies the mass states and splittings in the medium and alters the mixing 
(a) SNO Flux Measurement

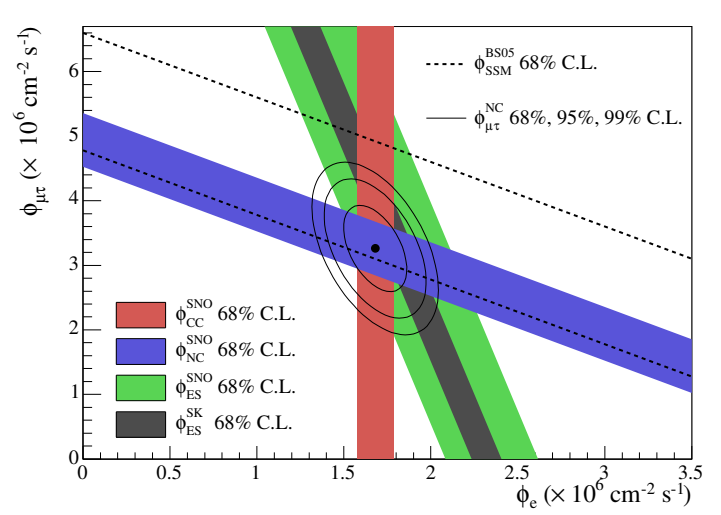

(b) Oscillation Results

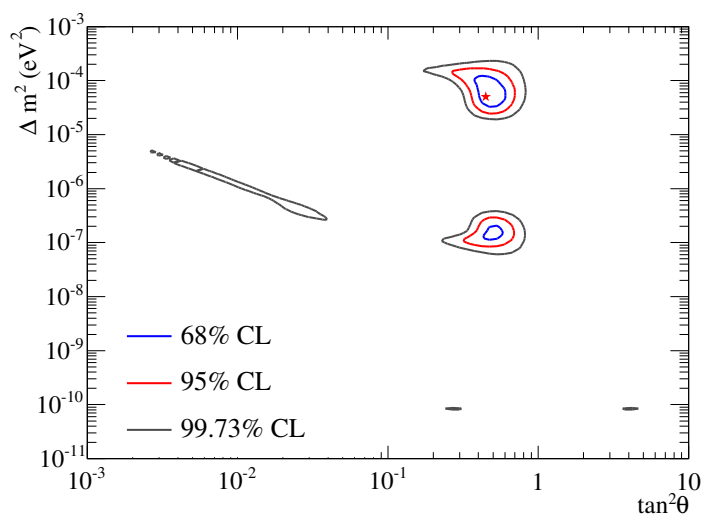

Figure 2.8: Results from the salt phase of the SNO experiment [33] in terms of the (a)measurement of $\nu_{\mu}+\nu_{\tau}$ flux vs $\nu_{e}$ flux and (b)Confidence limits for the oscillation parameters $\Delta m_{12}^{2}$ and $\theta_{12}$.

angles. The oscillation fit in Figure 2.8 $\mathrm{b}$ is consistent with all three of the oscillation solutions, but favors the LMA solution.

Global analyses of the solar neutrino experiments have also favored the LMA solution [36]. This piece of the neutrino oscillation puzzle has advanced far in the years, and much can yet be done. The radiochemical experiments have been able to detect sub$\mathrm{MeV}$ solar neutrinos, but can not collect real time solar neutrino data. The real-time solar neutrino experiments have not been able to reach low enough energy thresholds to measure the flux of p-p chain neutrinos, from the primary solar nuclear reaction. Future experiments in solar neutrino are seeking to make real-time measurement of the low-energy neutrino flux [37]. This will enable both more powerful measurements of neutrino oscillation parameters and solar nuclear-reaction rates.

\subsubsection{Atmospheric Neutrino Anomaly}

In the late 1970's and early 1980's, many experiments were searching for proton decay to test certain grand unified theories (GUTS) that predicted such a phenomenon. These experiments looked for the specific signature of a proton decay in the middle of a large amount of material. A proton decay would appear as a series of deeply-inner active detector elements hit without the outer active detector elements producing a hit to 
indicate a cosmic ray muon passing through the detector. A major background to the observation of proton decay was from atmospheric neutrinos that would occasionally interact in the middle of the detector and produce and proton-decay-like signature event. These were easy enough to distinguish from proton-decay events from the direction of the daughter particles. The particles from the neutrino interaction would mostly point forward along the direction that the neutrino was traveling, while a proton-decay particles should have a net vectorial momentum of zero. In the process of studying these background atmospheric neutrinos, physicist found anomalously low interaction rates.

Cosmic rays hitting Earth's atmosphere consist primarily of energetic protons and heavier nuclei. These interact in the atmosphere to produce both $\nu_{e}$ and $\nu_{\mu}$ in the chains $\pi^{ \pm} \rightarrow \mu^{ \pm}+\nu_{\mu}\left(\bar{\nu}_{\mu}\right)$ followed by $\mu^{ \pm} \rightarrow e^{ \pm}+\nu_{e}\left(\bar{\nu}_{e}\right)+\bar{\nu}_{\mu}\left(\nu_{\mu}\right)$. These decay modes produce $\sim 2$ times more $\nu_{\mu}$ than $\nu_{e}$. Atmospheric neutrino experiments have developed simulations to model the atmospheric neutrino flux [2, 38. These models quote uncertainties of $\sim 20 \%$ in the absolute neutrino flux [6], but are are able make better predictions of neutrino flux ratios. With detailed analyses of the cosmic-ray flux, hadron production, and geomagnetic effects on atmospheric neutrino production, the neutrino flavour ratio and up muon neutrino up-down ratio are predicted with uncertainties of $\sim 1$.

The IMB and Kamiokande experiments were the first to observe what would become known as the "atmospheric neutrino anomaly. These water Čerenkov detectors were able to distinguish electrons from muons based on the shape of their Čerenkov radiation cone, and measures the atmospheric $\nu_{\mu}$ and $\nu_{e}$ fluxes based on the charged-current interaction rates. These experiments calculated the "atmospheric neutrino flavor ratio $\left(\Re=\frac{R_{\nu_{\mu} / \nu_{e}}^{\text {data }}}{R_{\nu_{\mu} / \nu_{e}}^{M C}}\right)$, and both observed a deficit in this value [39, 40].

In addition to the water Cerenkov detectors, an additional class of proton-decay and atmospheric-neutrino detectors used iron plates combined with an active sampling element to detect the passage of particles through the detector. Two early such experiments, NUSEX and Frejus, failed to observe evidence for an anomalously low atmospheric neutrino flavor double ratio [41, 42]. The later experiments Soudan2 and MACRO found that it is necessary to surround the detector with a hermetic shielding to remove the background from cascades in the surrounding rock, which will obscure the effect of the atmospheric neutrino anomaly. The measurement of the $\Re$ performed by the Soudan 2 experiment in their $1 \mathrm{kt}$ calorimeter detector was consistent with the 
earlier measurement of Kamiokande and IMB [43. When Kamiokande was upgraded to Super-K, a much more precise measurement of $\Re$ was possible. The results of all of these measurements are shown in Figure 2.9.

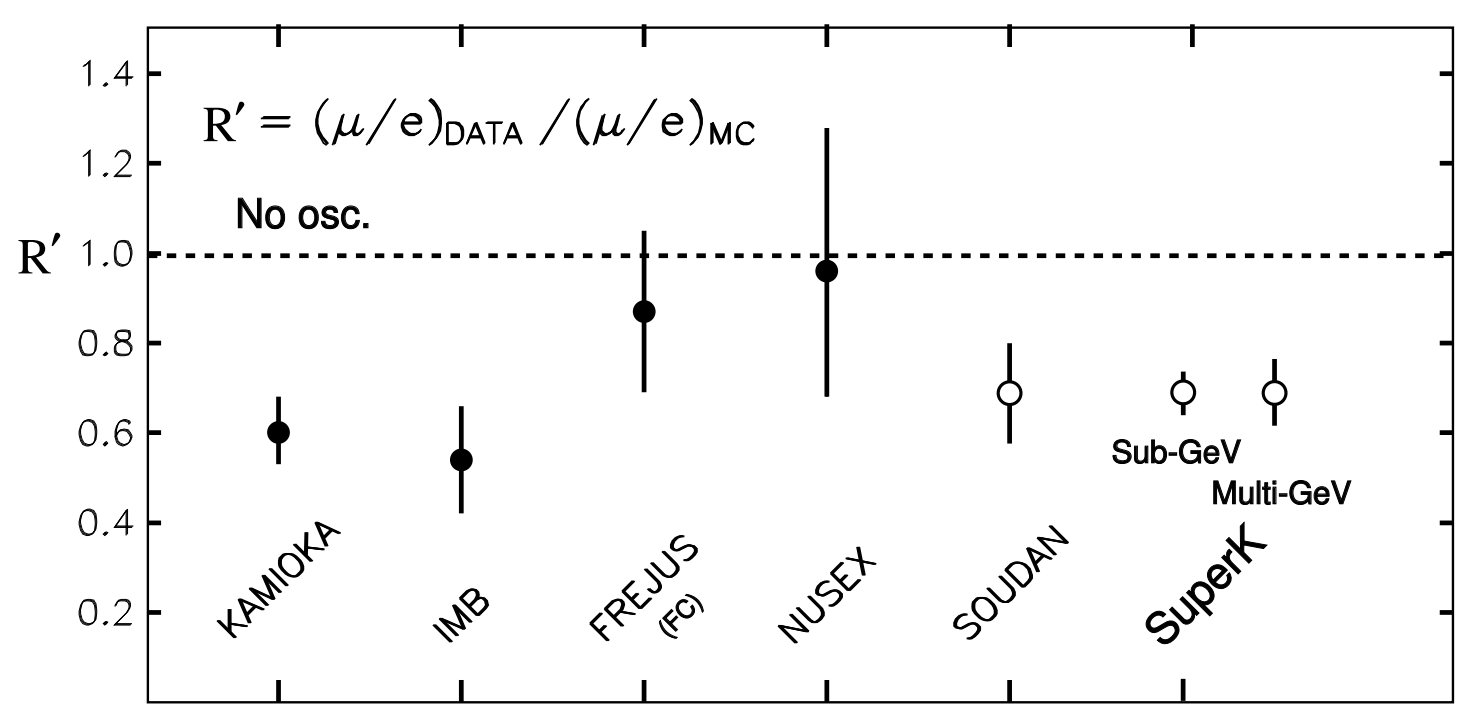

Figure 2.9: Various measurements made of the atmospheric neutrino double flavor ratio [44.

This low double flavor ratio could be interpreted as a deficit in the $\nu_{\mu}$ flux relative to expectation, or a excess in the $\nu_{e}$ flux relative to expected. Future experiments would seek to validate one of these two interpretations. The oscillations of atmospheric $\nu_{\mu}$ could explain the atmospheric neutrino anomaly. To test this hypothesis, measurements could be made of the neutrino energy and the distance the neutrino traveled from its creation point to the detector. The neutrino energy can be measured from the energy of the lepton and hadronic system resulting from a $\nu$ CC interaction, and the propagation distance can be estimated with the incident zenith angle. Downward pointing neutrinos will have traveled a short distance to the detector, and upward pointing neutrinos will have traveled a long distance to the detector.

Super-K analyzed the zenith-angle spectrum for $\mu$-like and e-like events, and found that the $\mu$-like have an up-down asymmetry that is not visible in the e-like [45]. This was a strong indication that the low double flavor ratio was due to a reduction in the $\nu_{\mu}$ flux. A more complete analysis on a 1489 live-day exposure of fully-contained (FC) and 
partially-contained (PC) $\mu$-like events in the Super-K detector found that oscillation of muon-type atmospheric neutrinos was in good agreement with the atmospheric neutrino anomaly [46]. The resulting L/E spectrum and oscillation analysis are shown in Figure 2.10 .

Super-K L/E Spectrum

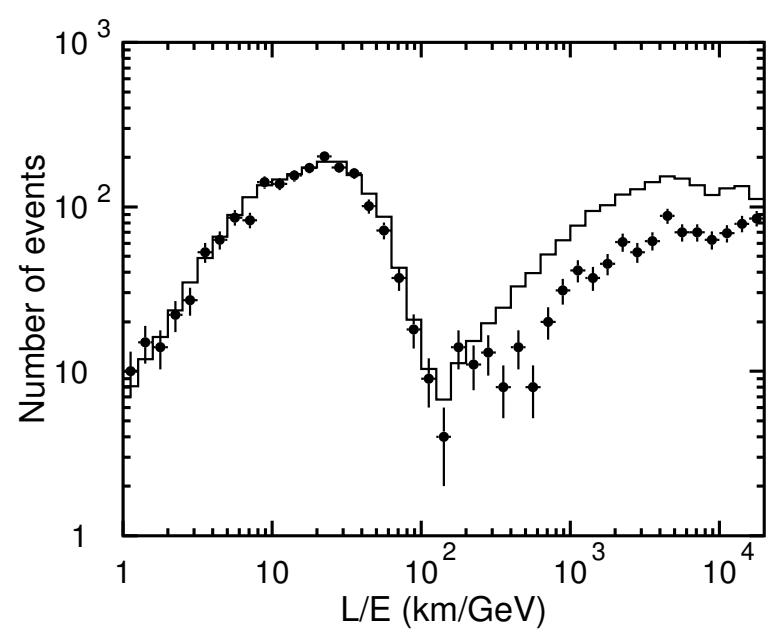

Resulting Oscillation Fit

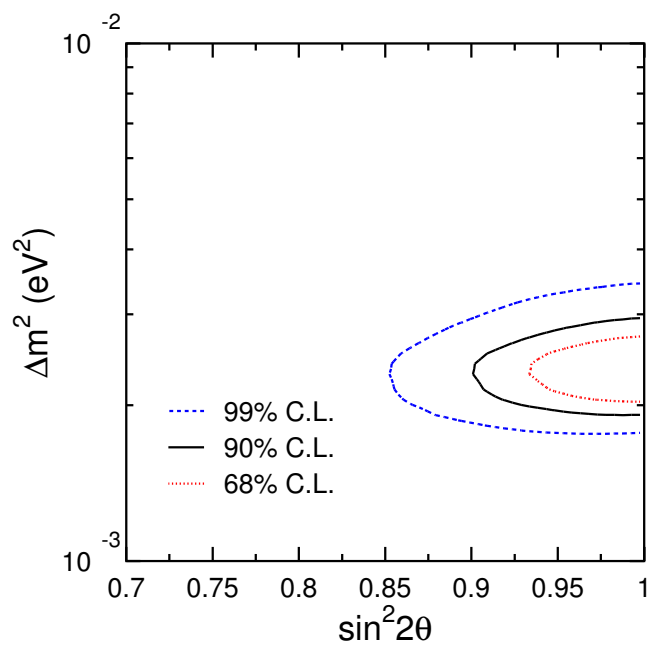

Figure 2.10: Super-K L/E observed and expected spectra and resulting allowed region for $\nu_{\mu} \rightarrow \nu_{\ell \neq \mu}$ oscillations [46].

The oscillation analysis of the Super-K L/E spectrum has determines best fit oscillation parameters $\Delta m_{23}^{2}$ and $\theta_{23}$ with two-flavour oscillations as described in Equation 2.33. The best point for this fit is at maximal mixing $\left(\sin ^{2}\left(2 \theta_{23}\right)=1.0\right)$ and $\Delta m_{23}^{2}=2.1 \times 10^{-3} \mathrm{eV}^{2}$. These latest results are dependent on $\nu_{\mu}$ disappearance oscillation, but doesn't answer the question, "what flavor is the muon neutrino oscillating into?" The e-like spectrum does not have an enhanced spectra where the mu-like spectrum is less than expected, so $\nu_{\mu} \rightarrow \nu_{e}$ oscillation is not a favored oscillation solution. Instead $\nu_{\nu} \rightarrow \nu_{\tau}$ is the favored oscillation solution. Charged-current $\nu_{\tau}$ interactions are not likely to be observed in Super-K because: (1) the energy threshold for $\nu_{\tau} \mathrm{CC}$ is $\sim$ $2 \mathrm{GeV}$ due to the mass of the $\tau$ and (2) a $\nu_{\tau} \mathrm{CC}$ is virtually indistinguishable from a $\nu$ $\mathrm{NC}$ interaction.

The Super-K experiment has instead observed the neutral-current interaction rate to distinguish the $\nu_{\tau}$ from the $\nu_{\text {sterile }}$ oscillation mode. All three flavor neutrinos have 
an equivalent $\mathrm{NC}$ interaction cross-section, but if a sterile neutrino existed it would not participate in any weak interactions (CC or NC). If $\nu_{\mu} \rightarrow \nu_{\text {sterile }}$ is occuring, the $\mathrm{NC}$ interaction rate would be low as well as the $\nu_{\mu} \mathrm{CC}$ rate. The findings from combining the low energy data, the high energy data, and the neutral current data favored a dominant oscillation mode of $\nu_{\mu} \rightarrow \nu_{\tau}$ at $99 \%$ confidence, but didn't rule out small mixing with a sterile neutrino [47].

\subsubsection{Reactor Neutrino Oscillation Experiments}

The intense source of neutrinos from a nuclear reactor was used to make the first neutrino detection. In the $1990 \mathrm{~s}$, physicist would also use these neutrinos to attempt the observation of $\bar{\nu}_{e}$ disappearance oscillation analogous to the $\nu_{e}$ disappearance oscillation observed with solar neutrinos. These experiments would have to use existing nuclear reactors, and place a $\bar{\nu}_{e}$ detector at an appropriate distance to optimize the $L / E$ of their measurement. Since the neutrino source from a reactor is isotropic, gains in $\Delta m^{2}$ sensitivity with increased $L$ are at the expense of loss in intensity at $\frac{1}{7} L^{2}$.

In the PMNS neutrino mixing matrix, the mixing angle $\theta_{13}$ can express sensitivity to both the $\Delta m^{2}$ value measured by atmospheric and solar neutrino experiments. Equations 2.26 and 2.27 demonstrate how both $\nu_{\mu}$ and $\nu_{e}$ disappearance are affected by $\theta_{13}$. The $\nu_{e}$ disappearance of solar neutrino is to strongly affected by the smaller $\Delta m^{2}$ term to observe any effect from $\theta_{13}$, and disappearance of atmospheric $\nu_{\mu}$ is overwhelmed by the potentially maximal mixing of $\theta_{23}$. The short-baseline disappearance of reactor neutrinos is sensitive to the atmospheric $\Delta m^{2}$ value, and no mixing angle term other than $\sin ^{2}\left(\theta_{13}\right)$ (Equation 2.29). Starting in the early 1980s, several experiments [48, 49, 50] tried to measure $\sin ^{2}\left(2 \theta_{13}\right)$, with no proof yet that this has a non-zero value [51]. The lowest bound to date on $\sin ^{2}\left(2 \theta_{13}\right)$ has been measured by the Chooz reactor neutrino experiment [52].

The Chooz experiment measured the flux $\bar{\nu}_{e}$ from the Chooz nuclear power plant in northern France with a 5-ton Gadolinium-doped liquid-scintillator detector. The detector observed the inverse beta decay process $\left(\bar{\nu}_{e}+p \rightarrow N+e^{+}\right)$by virtue of a prompt $e^{+}$annihilation signal followed by photon emissions from the capture of the $n$. The Gadolinium doping of the liquid scintillator was done to enhance the neutron capture efficiency and increase the energy of the emitted photons following neutron 
capture.

(a) Chooz Energy Spectrum
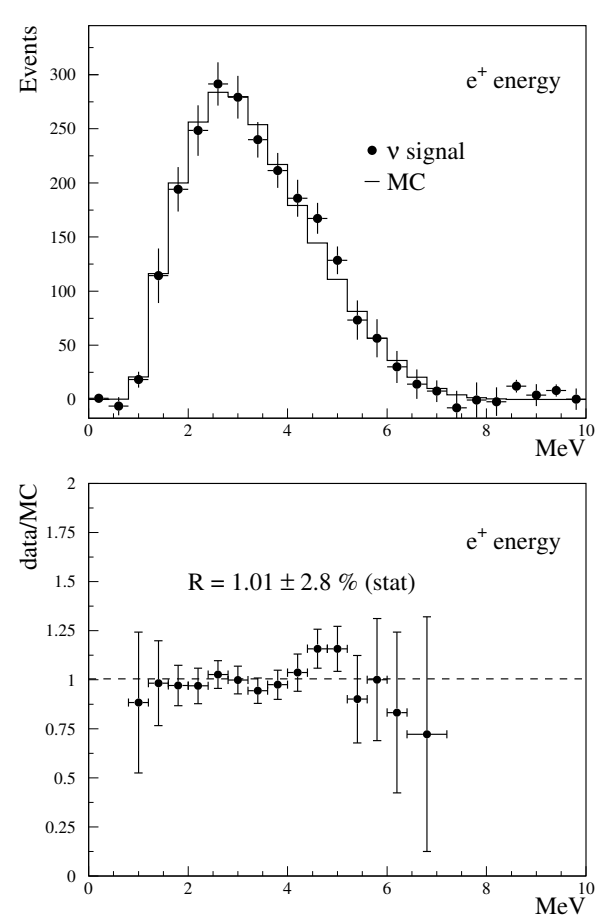

(b) Oscillation Exlcusion

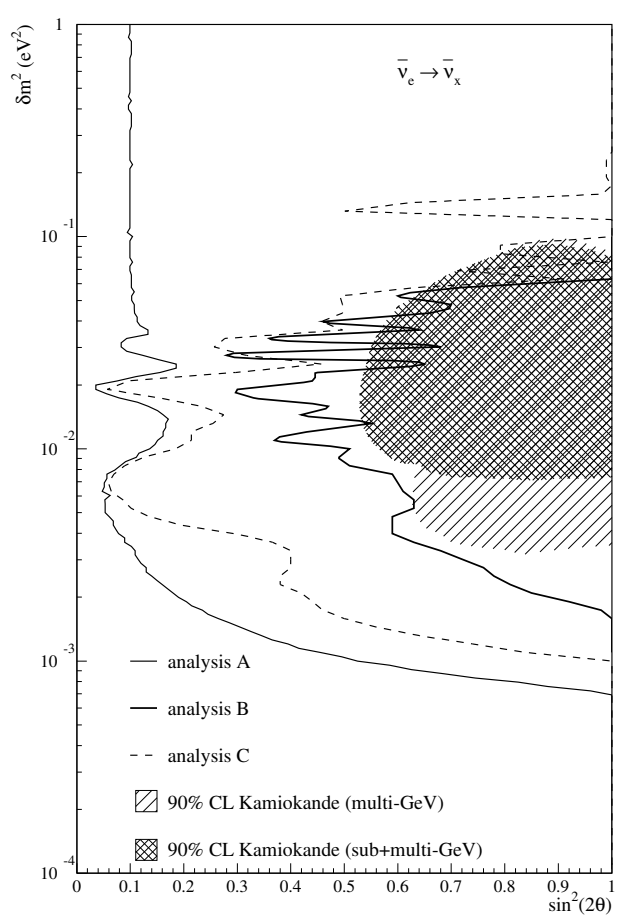

Figure 2.11: Result of the Chooz reactor-neutrino oscillation experiment [53].

Neutrino oscillation in Chooz experiment would be apparent as a $\bar{\nu}_{e}$ interaction rate deficit and a distortion relative to the null-oscillation prediction in the $\bar{\nu}_{e}$ spectrum. The expected and measured $\bar{\nu}_{e}$ spectra are shown in Figure 2.11 a, along with the ratio of the measured to expected spectra. The measured and expected spectra are in sufficient agreement to suggest no oscillation is being observed. A limit on oscillation parameters has been set with the result of this spectral comparison, shown in Figure 2.11] b. When the confidence limit from this oscillation fit is combined with the atmospheric neutrino oscillation results from Super-K (also shown on Figure 2.11 b), the Chooz experiment has been able to state with a $90 \%$ confidence limit that $\sin ^{2}\left(2 \theta_{13}\right)<0.17$ [53].

While the short-baseline reactor neutrino experiments sought to measure $\theta_{13}$, longbaseline reactor neutrino experiments could instead attempt to measure the same oscillation signal that has been observed in solar neutrinos. If CPT invariance holds, $\bar{\nu}_{e}$ 
oscillation results from nuclear reactor neutrinos can be combined with the $\nu_{e}$ oscillation results from solar neutrinos to constrain oscillation parameters. The KamLAND long-baseline reactor neutrino experiment [54] has been measuring the $\bar{\nu}_{e}$ flux since 2001 from 16 nuclear power plants in Japan. The reactors are from $80 \mathrm{~km}$ to $800 \mathrm{~km}$ from the Kamiokande mine, where the $\bar{\nu}_{e}$ are detected with a $1 \mathrm{kT}$ liquid scintillator detector. The KamLAND detector observes the inverse beta decay process from $\bar{\nu}_{e}$ $\left(\bar{\nu}_{e}+p \rightarrow N+e^{+}\right)$, much as the Chooz experiment had done. Figure 2.12 shows the schematic view of this detector, along with the spectra of the prompt and delayed photon inverse beta decay signature.

\section{KamLAND Detector}

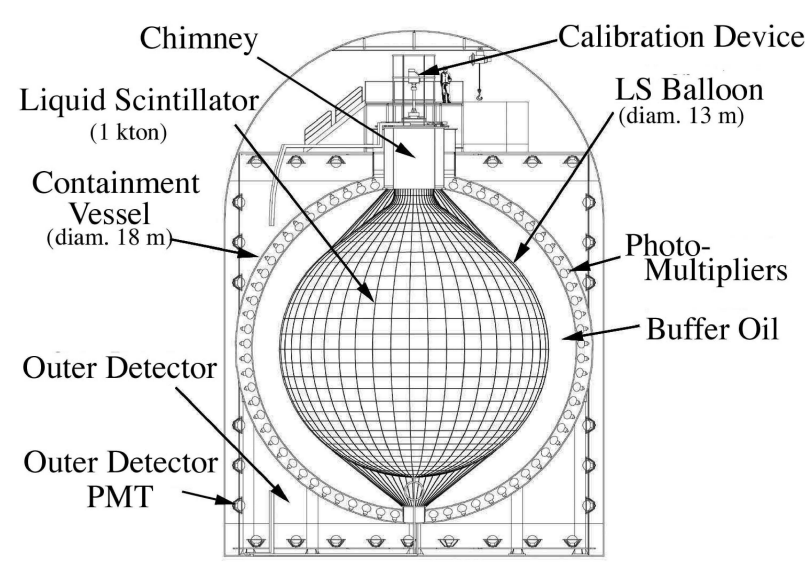

\section{Reactor Neutrino Spectrum}

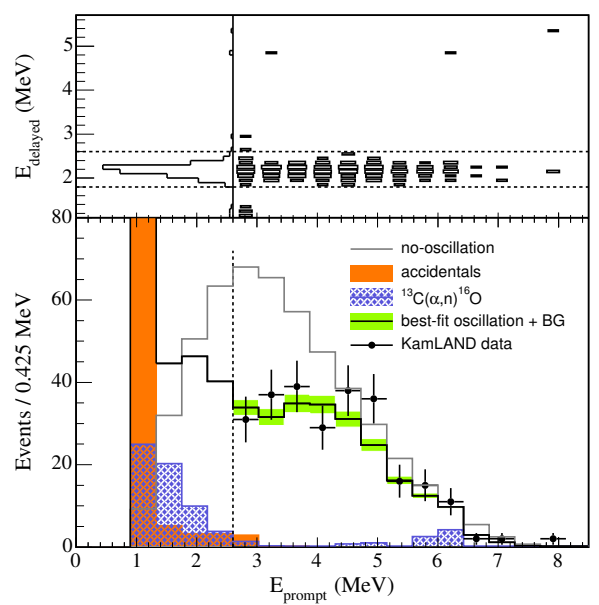

Figure 2.12: A schematic view of the KamLAND detector [55], and the energy spectra of prompt $e^{+}$photon from $\bar{\nu}_{e}$ interactions and delayed photon from neutron capture [56]. The distortion in the measured spectrum relative to the unoscillated expectation is significant.

The $e^{+}$prompt energy spectra of a 766 ton-year exposure in KamLAND has been analyzed for neutrino oscillation [56]. The spectra shown in Figure 2.12 have been analysed assuming two-flavour oscillations to determine the $\Delta m_{12}^{2}$ and $\theta_{12}$ oscillation parameters. Unlike solar neutrino neutrinos, the reactor neutrino will not experience a significant MSW matter effect to enhance oscillation, which simplifies the neutrino oscillation analysis. Figure 2.13 a shows the confidence limits for $\Delta m_{12}^{2}$ and $\theta_{12}$ for along 
with the global solar neutrino confidence limits. KamLAND oscillation confidence limits are consistent with the LMA solution to the solar neutrino anomaly. Figure 2.13 b shows the confidence limits from combining KamLAND results and the global solar neutrino analysis. The best fit point on this graph occurs at $\Delta m_{12}^{2}=7.9_{-0.5}^{+0.6} \times 10^{-5} \mathrm{eV}^{2}$, $\tan ^{2}\left(\theta_{12}\right)=0.40_{-0.07}^{+0.10}$

\section{(a) Oscillation Fit of KamLAND KamLAND Prompt Spectrum}

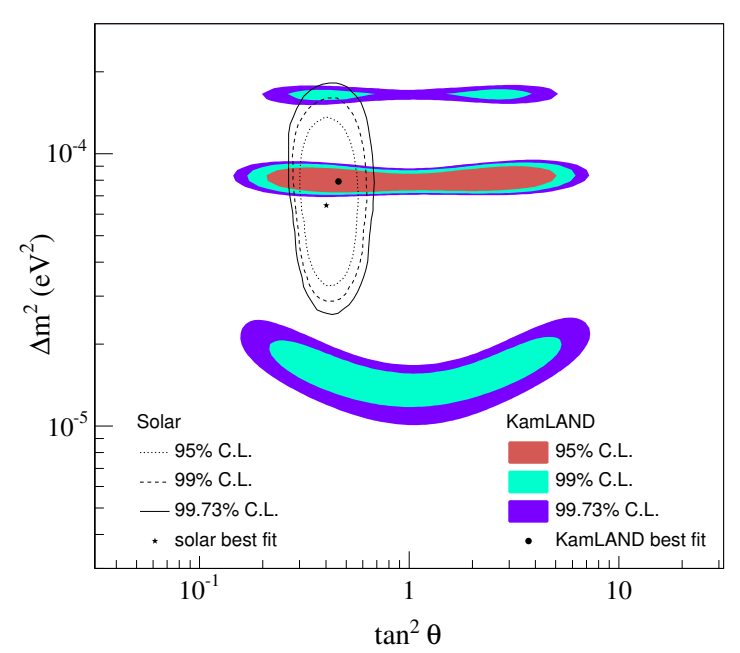
(b) Combined Oscillation Fit
KamLAND + Solar Data

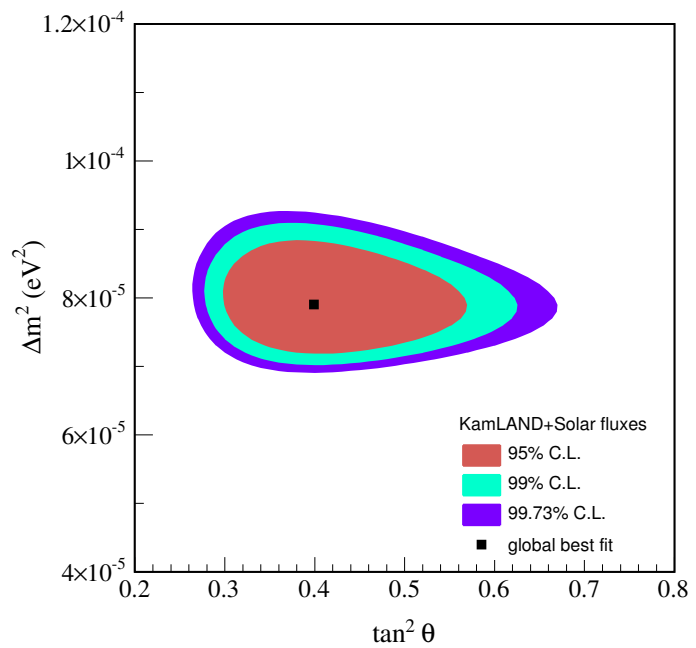

Figure 2.13: Oscillation parameter analysis of (a) KamLAND prompt energy spectrum and (b) combined global analysis of solar neutrino and KamLAND spectra [56].

\subsubsection{Accelerator Beam Neutrino Oscillation Experiments}

Later generations of neutrino oscillation have sought to control the neutrino source in order to perform precision measurements of oscillation parameters. High energy particle accelerators can make a neutrino beam much the way that cosmic-rays make neutrino beam out of the atmosphere. Protons hit a target (air molecules for atmospheric neutrinos and some heavy element for accelerator neutrinos), and pions are produced that decay into $\mu$ and $\nu_{\mu}$. Since the decay length of the pion is energy dependent, the neutrino energy can be controlled with energy of the incident protons, and the allowed pion decay length. 
The Liquid Scintillator Neutrino Detector (LSND) short-baseline accelerator neutrino experiment has reported an additional, unconfirmed oscillation signal [57]. The LSND detector, located at the Los Alamos Meson Physics Facility (LAMPF), observed a neutrino beam with a 160 ton liquid scintillator detector. The neutrino beam is produced with $800 \mathrm{MeV}$ protons dumped onto a fixed target, with the detector is situated $30 \mathrm{~m}$ away. Many of the secondary $\pi^{+}(\sim 99 \%)$ come to rest and decay to $\mu^{+}$and $\nu_{\mu}$, and most of the $\mu^{+}$also decay at rest and by $\mu^{+} \rightarrow e^{+}+\nu_{e}+\bar{\nu}_{\mu}$.

LSND reported an excess of $87.9 \pm 22.4 \pm 6.0 \bar{\nu}_{e}$ events for neutrino energies in the range $E \simeq 36-60 \mathrm{MeV}$, which they interpreted as resulting from $\bar{\nu}_{\mu} \leftrightarrow \bar{\nu}_{e}$ neutrino oscillation [58. This result has been considered questionable, as other shortbaseline neutrino oscillation experiments have not observed this $\bar{\nu}_{e}$ appearance signal. Figure 2.14 shows the allowed oscillation parameter regions resulting from LSND, the KARMEN2 [59] accelerator neutrino experiment, the CCFR experiment [60] and the Bugey [50] reactor neutrino experiment. KARMEN2 and Bugey observed $\bar{\nu}_{e}$ interaction rates that were in good agreement with the expected interaction rates. Much of the LSND region is excluded by the results of other experiments.

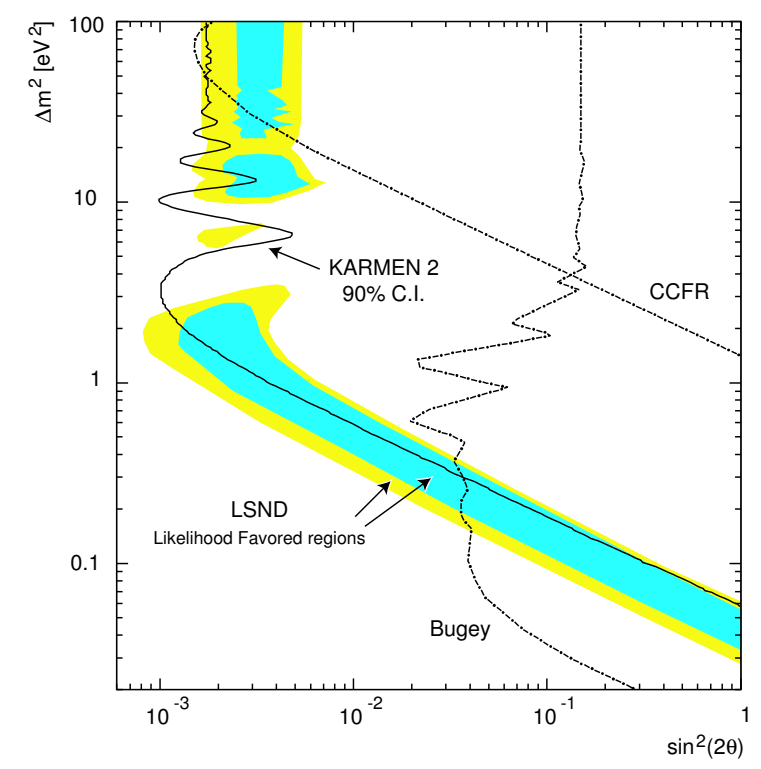

Figure 2.14: Comparison of results from several short baseline neutrino oscillation experiments [59]. 
If the measurement that LSND has made is correct, it would present the possibility of a third $\Delta m^{2}$ value. The three neutrino scheme allows only two distinct $\Delta m^{2}$ values, and measurements made at LEP have shown that there are only 3 lepton flavors that participate in the weak interaction [19]. To explain the results from LSND, a "sterile" neutrino may be introduced, which does not couple to the weak force. To independently verify the LSND result, the MiniBooNE experiment has been constructed at Fermilab 61]. This experiment will be sensitive to oscillations in LSND's favored oscillation parameter region.

The short-baseline neutrino oscillation experiments are limited by their systematic understanding of the neutrino beam. To alleviate this, long-baseline neutrino oscillation experiments operate with a near and a far detector to produce two independent measurement of neutrino beam. This type of experiment can make a precise measurement of the atmospheric neutrino oscillation parameter space. Recently, the K2K long-baseline accelerator neutrino experiment [62] has performed such a measurement.

The K2K experiment uses a neutrino beam from the KEK accelerator facility in Tsukuba, Japan. A $12 \mathrm{GeV}$ proton beam is dumped onto a fixed aluminium target, producing an intense flux of secondary pions and kaons. The positive secondary hadrons are focused with a pair of magnetic horns, and aimed down a $200 \mathrm{~m}$ decay pipe. The result is a $98 \%$ pure $\nu_{\mu}$ beam with a $1.3 \mathrm{GeV}$ mean energy. The primary $\nu_{\mu}$ beam spectrum is measured $300 \mathrm{~m}$ from the proton target with a $1 \mathrm{kt}$ water Cerenkov detector combined with a system of fine-grained detectors. The beam is then measured by the Super-K detector, $250 \mathrm{~km}$ away. Neutrinos from the beam are easily distinguished from cosmic-ray muons or atmospheric neutrino by the pulsing structure of the beam. The expected neutrino spectrum in the Super-K from the neutrino beam is determined by extrapolating the neutrino spectrum in the near detectors.

K2K has measured a few events than expected at SKK, observing 107 events compared to the expected number of $151_{-10}^{+12}$ [63. A sample of high-resolution events have been chosen to measure the beam energy spectrum. These are events where the energy can be reconstructed from from the measured muon energy and direction with charged current quasi-elastic kinematics. Figure 2.15] a shows the energy spectrum next to the unoscillated prediction and the best fit oscillated spectrum. The spectral distortion is used to perform a two-flavour muon disappearance neutrino oscillation analysis. 
Figure 2.15] b shows the confidence limits obtained with the oscillation analysis of the K2K spectral deficit. The confidence contours here are consistent with those from the atmospheric neutrino analyses by Super-K and other experiments.

(a) K2K Spectrum

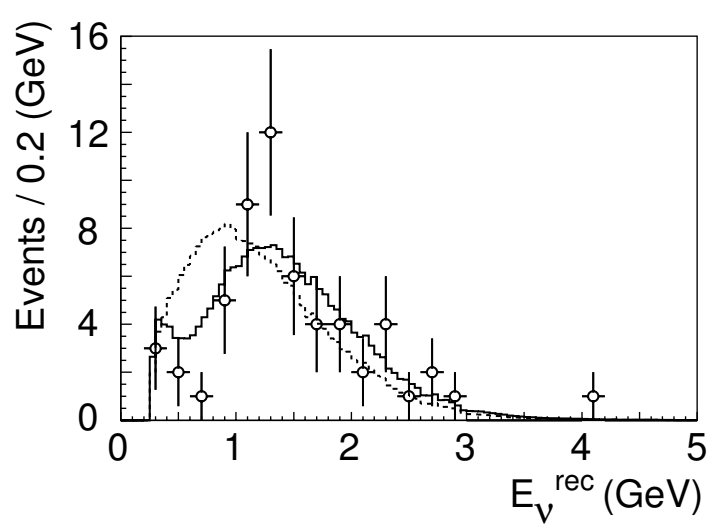

(b) Oscillation Fit

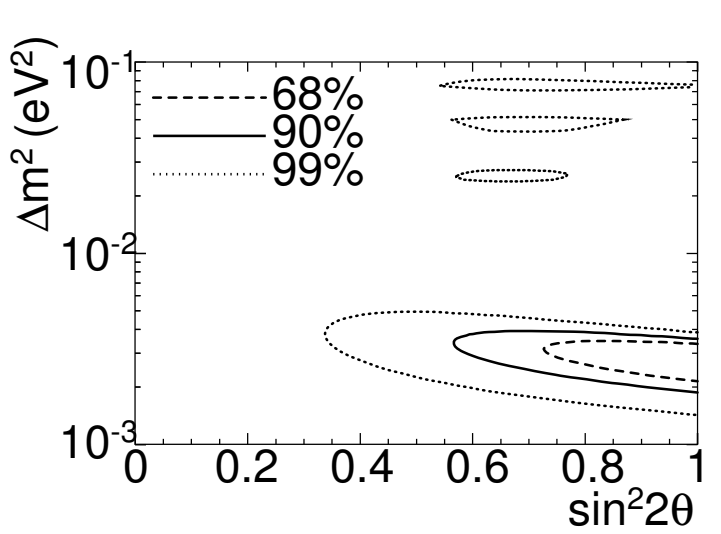

Figure 2.15: (a) Reconstructed energy spectrum of $\nu_{\mu} \mathrm{CC}$ events in $\mathrm{K} 2 \mathrm{~K}$, and (b) K2K allowed region for $\nu_{\mu} \rightarrow \nu_{\tau}$ oscillations 63.

\subsubsection{Neutrino Oscillation Experimental Status}

Oscillation experiments have confirmed two distinct channels of oscillation with solar / reactor neutrino experiments $\left(\Delta m_{12}^{2} \simeq 8^{-5} e V^{2}, \tan ^{2}\left(\theta_{12}\right) \simeq 0.4\right)$, and atmospheric / accelerator neutrino experiments $\left(\Delta m_{23}^{2} \simeq 10^{-3} \mathrm{eV}^{2}, \sin ^{2}\left(2 \theta_{23}\right) \simeq 1.0\right)$. The third independent mixing angle has been constrained by the results from Chooz to $\sin ^{2}\left(2 \theta_{13}\right) \leq 0.17$. The mixing angles can be approximated to be $\theta_{23}=\frac{\pi}{4}, \theta_{12}=\frac{\pi}{6}$, and $\theta_{31}<0.21$. The component mixing matrices can be expressed as:

$$
\begin{gathered}
\frac{U_{12}}{\Downarrow} \\
U=\left(\begin{array}{ccc}
\frac{\sqrt{3}}{2} & \frac{1}{2} & 0 \\
-\frac{1}{2} & \frac{\sqrt{3}}{2} & 0 \\
0 & 0 & 1
\end{array}\right)\left(\begin{array}{ccc}
1 & 0 & 0 \\
0 & \frac{1}{\sqrt{2}} & \frac{1}{\sqrt{2}} \\
0 & -\frac{1}{\sqrt{2}} & \frac{1}{\sqrt{2}}
\end{array}\right)\left(\begin{array}{ccc}
>0.98 & 0 & (<0.21) \times e^{-i \delta} \\
0 & 1 & 0 \\
(<0.21) \times-e^{i \delta} & 0 & >0.98
\end{array}\right)
\end{gathered}
$$

In the limit that $\theta_{13} \approx 0$, the mixing matrix is written: 


$$
U \approx\left(\begin{array}{ccc}
\frac{\sqrt{3}}{2} & \frac{1}{2} & 0 \\
-\frac{1}{2 \sqrt{2}} & \frac{\sqrt{3}}{2 \sqrt{2}} & \frac{1}{\sqrt{2}} \\
\frac{1}{2 \sqrt{2}} & -\frac{\sqrt{3}}{2 \sqrt{2}} & \frac{1}{\sqrt{2}}
\end{array}\right)
$$

Future experiments in neutrino oscillation have many questions to answer regarding the nature of the neutrino mass and mixing. The final angle in the mixing matrix $(\theta)$ is being sought with experiment searching for $\nu_{\mu} \leftrightarrow \nu_{e}$ oscillation. If this angle is nonzero, then the possibility of $\mathrm{CP}$ violation in lepton mixing with the $\delta$ phase term will be the next important piece of the PMNS matrix. Solar neutrino experiments have been able to determine the sign of $\Delta m^{2}{ }_{12}$, but muon-disappearance experiments have to determine the sign of $\Delta m^{2} 23$. 


\section{Chapter 3}

\section{MINOS Experiment}

With the success of the Soudan2 experiment, the Soudan Underground Lab was proven as an excellent location to do deep-underground low-background physics. Oscillation studies of atmospheric neutrinos contributed much to the knowledge of neutrino mass and mixing in the 1980s and 1990s, but contribution atmospheric neutrino studies was quickly becoming limited. Two limitations of atmospheric neutrinos would drive the next stage in neutrino oscillation studies: (1) the spectral flux of neutrinos can only be understood theoretically, and (2) the large number of electron neutrinos among the atmospheric neutrinos complicates the analysis of muon neutrinos oscillating into electron neutrinos. By creating a neutrino beam, the flux of neutrinos could be measured with a detector immediately in front of the beam, and the contamination of electron neutrinos can be minimized by beam design.

The MINOS (Main Injector Neutrino Oscillation Search) project was conceived largely by members of the Soudan2 collaboration in the early 1990s. A beam of neutrinos would be created at Fermilab with protons from the main injector. There would be a "near detector" directly in front of the beam in a shallow cavern. The near detector would have a high enough flux of neutrinos that it would not have be deep underground to filter cosmic rays. A "Far Detector" would be constructed in the Soudan Mine, where the shielding from the cosmic-ray muons would offer a cleaner spectral measurement of the neutrino beam 64.

Figure 3.1 demonstrates the layout of the MINOS experiment. The three stages of the MINOS experiment are presented in this chapter. Special detail is paid to the far 

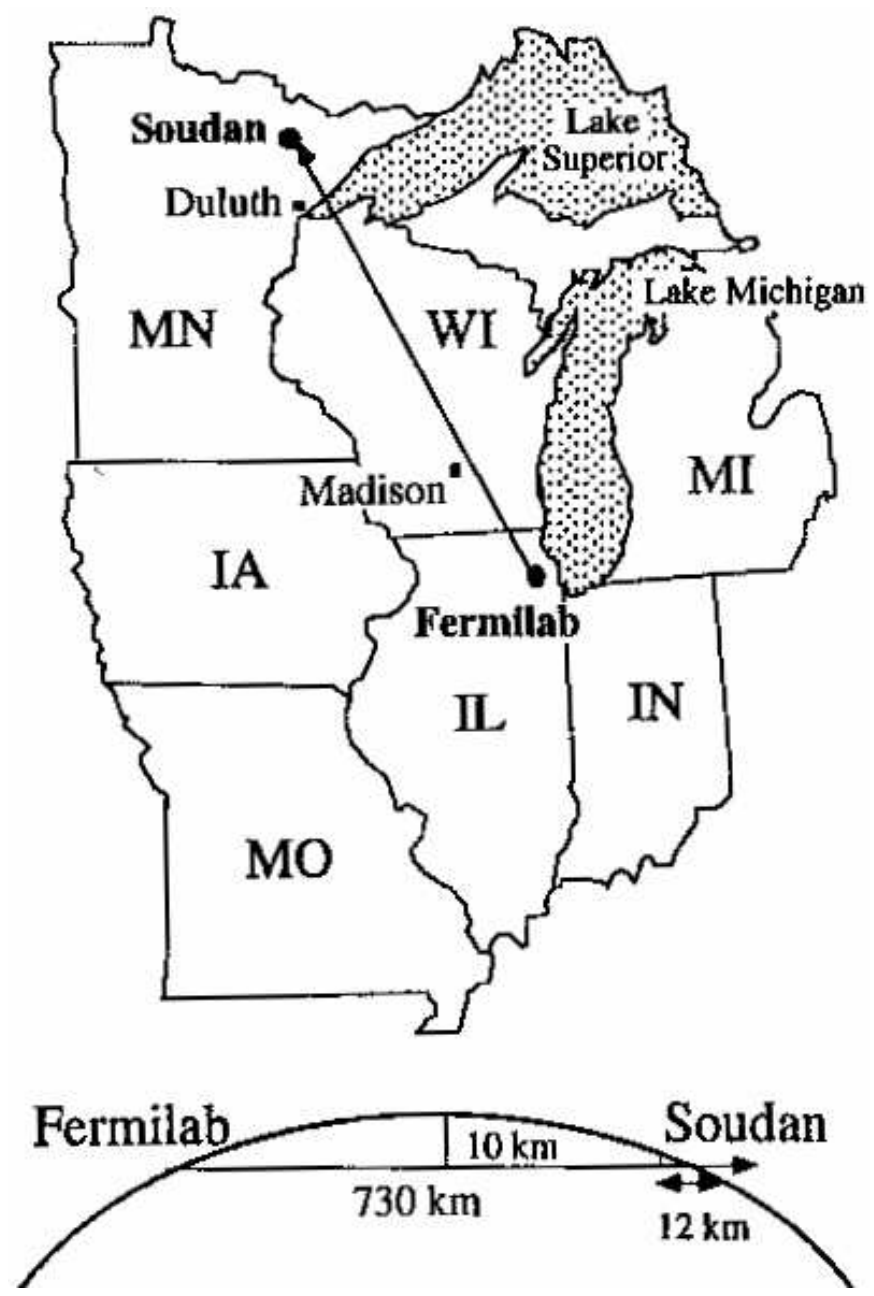

Figure 3.1: The layout of the MINOS experiment, from the NuMI beam and near detector at Fermilab to the far detector in the Soudan Mine [64. 
detector, as this is the instrument that collected the data used in this thesis.

\subsection{NuMI Beam}

The NuMI Beam is constrained by the primary demands of intensity, neutrino type purity, energy range, and direction. The beam intensity must be high enough to produce a statistically significant number of neutrino interaction in the detectors. The beam must have a minimal number of electron neutrino to be able to detect $\nu_{\mu} \rightarrow \nu_{e}$ oscillation. The beam neutrino energy range must contain energies at which the muon neutrino is sensitive to oscillation. The beam direction must point directly at the mine.

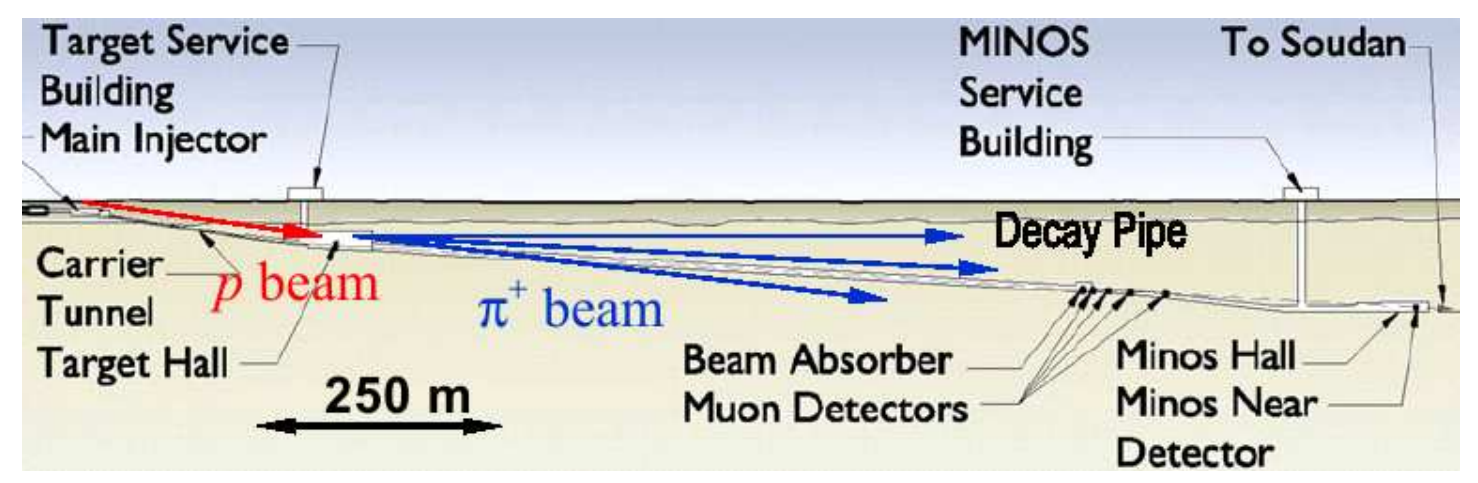

Figure 3.2: Side view of the Neutrinos at the Main Injector (NuMI) beam line.

The main injector at the Fermi National Accelerator Laboratory (FNAL) is one of the staging rings in a series of proton accelerators before final stage proton / anti-proton collider, the Tevatron. To create the NuMI beam, $120 \mathrm{GeV}$ protons are extracted from the main injector beam line and magnetically aimed downward. Each batch extraction is referred to as a "beam spill", which contains $4 \times 10^{13}$ protons at $120 \mathrm{GeV}$. The extraction from the main injection lasts $\sim 8 \mu \mathrm{s}$, and occurs at a rate of $0.53 \mathrm{~Hz}$.

The extracted proton beam is aimed a segmented graphite target. When the protons hit the graphite atoms, they interact much like high energy cosmic rays hitting the upper atmosphere. A stream of secondary pions and kaons is produced that will eventually decay and produce a beam of neutrinos. The focus the stream of pions back into their intended direction, two magnetic horns are stationed after the target (Figure [3.3). The horns are concentric inner and outer cylindrical conductors that are pulsed with a 200 


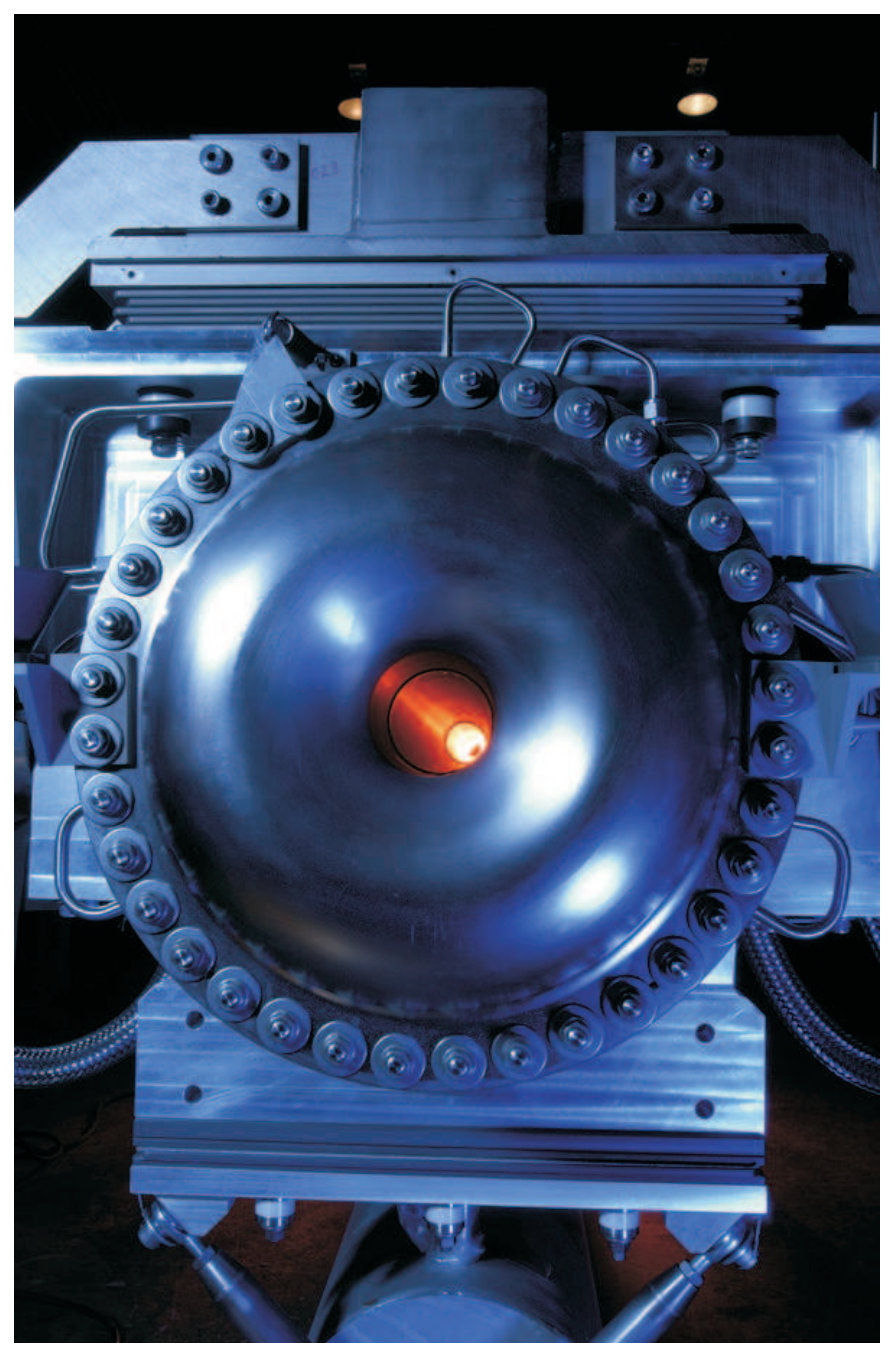

Figure 3.3: The NuMI magnetic horn, which sits a distance from the target to focus the pion stream resulting from the proton hitting the target. 
$\mathrm{kA}$ current in time with the beam to produce a $30 \mathrm{kG}$ toroidal magnetic field. The direction of the pulse is used to focus one sign of particles and defocus the opposite sign of particles. If positive particles are focused, the neutrino beam will be primarily $\nu_{\mu}\left(\pi^{+} \rightarrow \mu^{+}+\nu_{\mu}\right)$, while if negative particles are focused the beam will be primarily $\bar{\nu}_{\mu}\left(\pi^{-} \rightarrow \mu^{-}+\bar{\nu}_{\mu}\right)$. The momentum of the focused pions is dictated by the distance between the horn and the target. The target is rail mounted, which allows $2.5 \mathrm{~m}$ of freedom for the horn / target distance for fine tuning of the beam energy.

After the horns, the focused pions and kaons must have time to decay in order to produce a beam of neutrinos. To this end, they travel $675 \mathrm{~m}$ through a decay pipe where the pions decay $\left(\pi^{+} \rightarrow \mu^{+}+\nu_{\mu}\right.$ at $\left.99.99 \%\right)$ and the kaons decay $\left(K^{+} \rightarrow \mu^{+}+\nu_{\mu}+\pi^{\prime} s\right.$ at $66.76 \%, K^{+} \rightarrow e^{+}+\nu_{e}+\pi^{\prime} s$ at $4.98 \%$, and $K^{+} \rightarrow \pi^{\prime} s$ at $\left.28.27 \%\right)$. One goal of the neutrino beam is to achieve a pure a sample of $\nu_{\mu}$ as possible, but a small contamination of $\nu_{e}$ does come from the kaon decays. Another potential source of neutrino beam contamination is from muon decays $\left(\mu^{+} \rightarrow e^{+}+\bar{\nu}_{\mu}+\nu_{e}\right)$. At the end of the decay tunnel is a beam dump of steel and concrete, where the remaining kaons, pions, and muons are absorbed to leave only a beam of pure neutrinos.

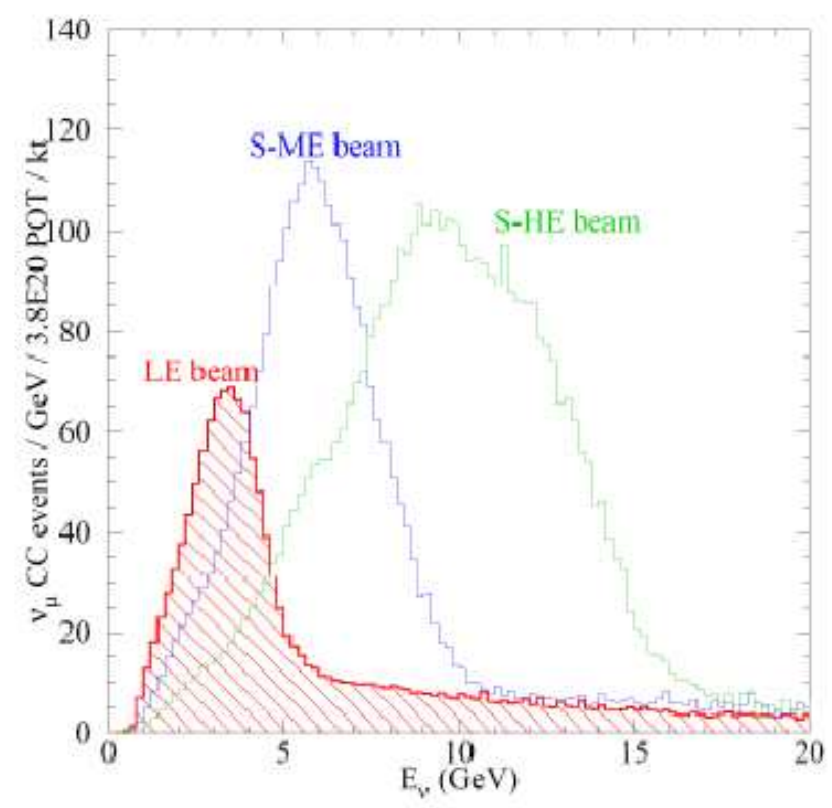

Figure 3.4: The three neutrino beam spectra for different target positions. 
The configuration of the distance between horns and target have been designed to allow for three separate beam running modes: High Energy (HE) for maximum horn and target separation, Medium Energy (ME) for closer horn and target separation, and Low Energy (LE) for the horns sitting adjacent and the target inside of the first horn. The neutrino beam spectra of these three configurations are shown in Figure 3.4 For more information on the beam and monitoring see references from Kopp [65], and Indurthy 66].

\subsection{MINOS Detectors}

The MINOS detectors were designed as muon calorimeters optimized to detect muons with a trajectory along the beam line. A typical modern muon calorimeter is comprised of dense absorber material sections that are separated by an active tracking element. Both detectors use steel plates as the dense absorber, and plastic scintillators as the active tracking element. A magnetic field is applied to both detectors to differentiate between the signs of charged particles. The near detector measures the neutrino beam spectrum for comparison to the spectrum in the far detector. In order for these spectra to have a consistent systematic comparison, the detector must be as similar as possible.

\subsubsection{Near Detector}

The near detector sits a short distance from the end of the NuMI decay pipe, about 1 $\mathrm{km}$ down stream from the NuMI target and $100 \mathrm{~m}$ underground. The neutrino beam spot when it reaches the near detector is $\sim 50 \mathrm{~cm}$. The design of the near detector is optimization based on the knowledge that there will be a higher flux of neutrinos in a smaller area, as compared to the far detector [64].

The 282 steel planes in the near detector are each $2.54 \mathrm{~cm}$ thick and measure $3.8 \mathrm{~m}$ by $4.8 \mathrm{~m}$. The full mass of the detector is $0.94 \mathrm{kton}$. Figure 3.5 a shows the face view of this detector. The diamond hole contains the coil used to generate the magnetic field. The area to the left of this is the instrumented region where the neutrino beam strikes the detector face. Figure 3.5.b shows how the near detector is divided into four separate regions that server different functions. 
(a) Near Detector Face View

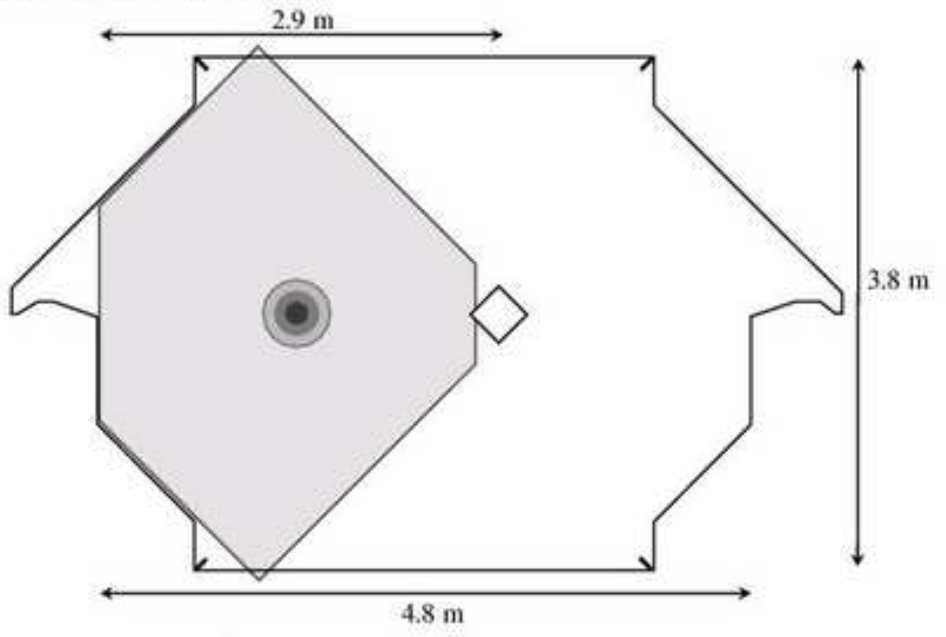

(b) Near Detector Side View

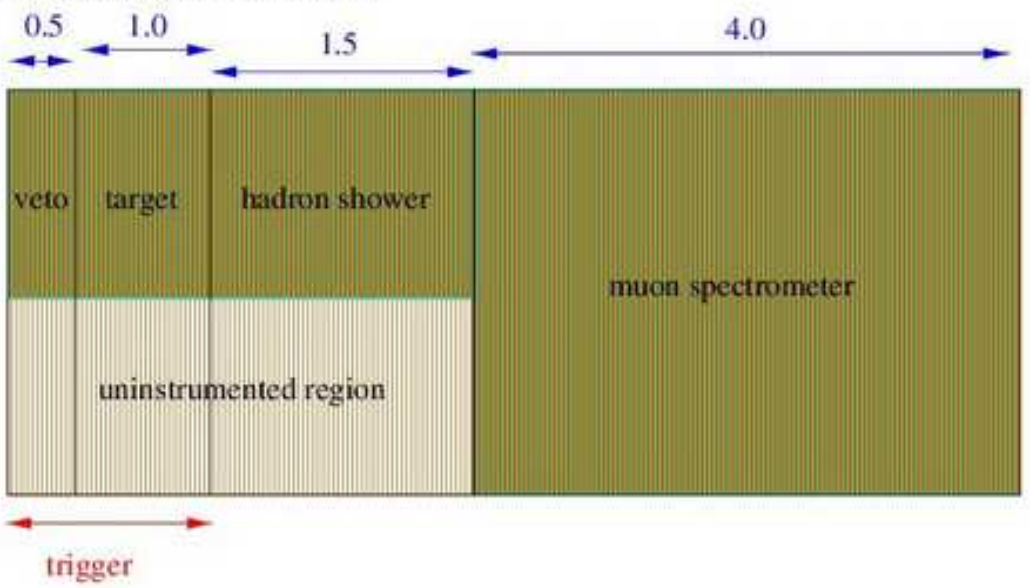

Figure 3.5: Schematic views of the MINOS near detector looking (a) face one and (b) from the side. 
1. Veto Region - First $0.5 \mathrm{~m}$ of near detector, used to remove events entering from the rock.

2. Target Region - Next $1.0 \mathrm{~m}$ of near detector, provides the fiducial area for neutrino interactions.

3. Shower Region - Next $1.5 \mathrm{~m}$ of near detector, measures the hadronic shower that accompanies a $\nu_{\mu}$ CC DIS interaction.

4. Spectrometer Region - Final $4.0 \mathrm{~m}$ of near detector, only instrumented every five planes, and used to measure the energy of the muon from the $\nu_{\mu} \mathrm{CC}$ interaction.

With each beam spill, it is expected that there will be $\sim 20$ neutrino interactions. With this many overlapping events, multiplexing or optical summing of events would not be a rational approach, and so each fiber has its own readout channel. For more information regarding the electronics readout see reference [67].

\subsubsection{Far Detector}

The MINOS far detector is located in the Soudan Underground Lab, in Soudan, MN. The detector is divided into two sections, called supermodules, and the whole detector is presented as the following conglomeration of elements:

- Steel - 486 Octagonal steel plates present a large amount mass for neutrinos to interact with, and a precise absorber material to slow muons.

- Magnetic Field - A coil is wrapped about the center of each supermodule to create a magnetic field to bend the path of muons, and offer both charge separation and momentum measurement.

- Scintillator and Fiber - All but two steel plates have one face covered with 192 scintillator strips that emit light a charged particle as it passes through the strip. The light is collected in a fiber glued down the center of the strip.

- Cosmic-Ray Veto Shield - To reject the background from cosmic-ray muons, modules of scintillator strips are mounted above the detector. 
- Photon Measurement and Optical Summing - Fibers from scintillator strips are readout at both ends by 16 pixel PMTs, where 8 fibers are readout by a single pixel.

- Electronics and Data Acquisition - Integrated circuits read the signal from the PMT and provide a trigger that decides when an interesting event is occurring in the detector. The signals all over the detector are accumulated by the data acquisition program and written to data files.

(a) MINOS Far Detector

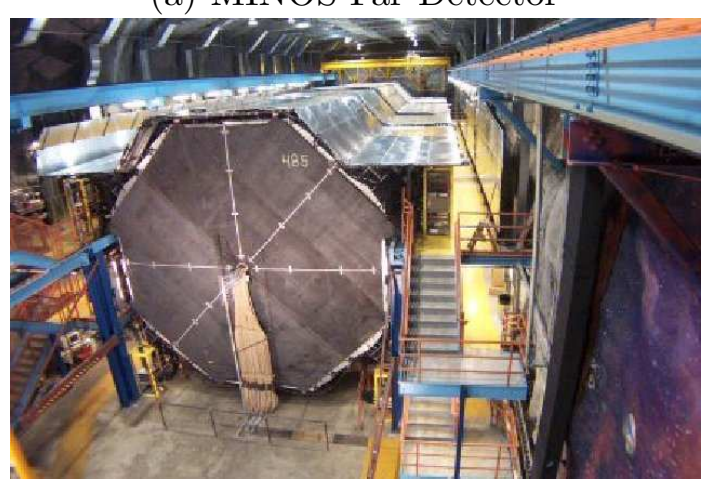

(b) Detector Coordinates

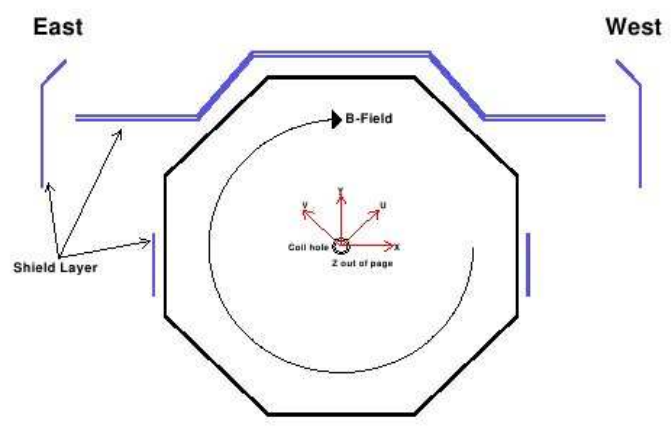

Figure 3.6: A view of the MINOS far detector facing south, towards Fermilab and the NuMI beam line [68].

\section{- Far Detector Steel -}

Both the far and near detectors use iron for absorber material to induce neutrino interactions and cause the resulting leptons to loose energy in a predictable fashion. A single far detector steel plane is assembled from 8 individual sections of steel, as shown in Figure 3.7. Four of the sections are lain flat on a strong-back support system, and aligned to form the detector octagonal structure. The other four are then lain on top of the previous four section in an identical octagonal structure, with the section joints at a $90^{\circ}$ angle to section joints in the lower set of steel sections. The set of planes are then welded together at multiple points to form a single, solid octagonal steel plate.

The strong-back support that was used as a platform to assemble the steel planes was then used to lift the plane with an overhead crane, and mount it on the rails in 

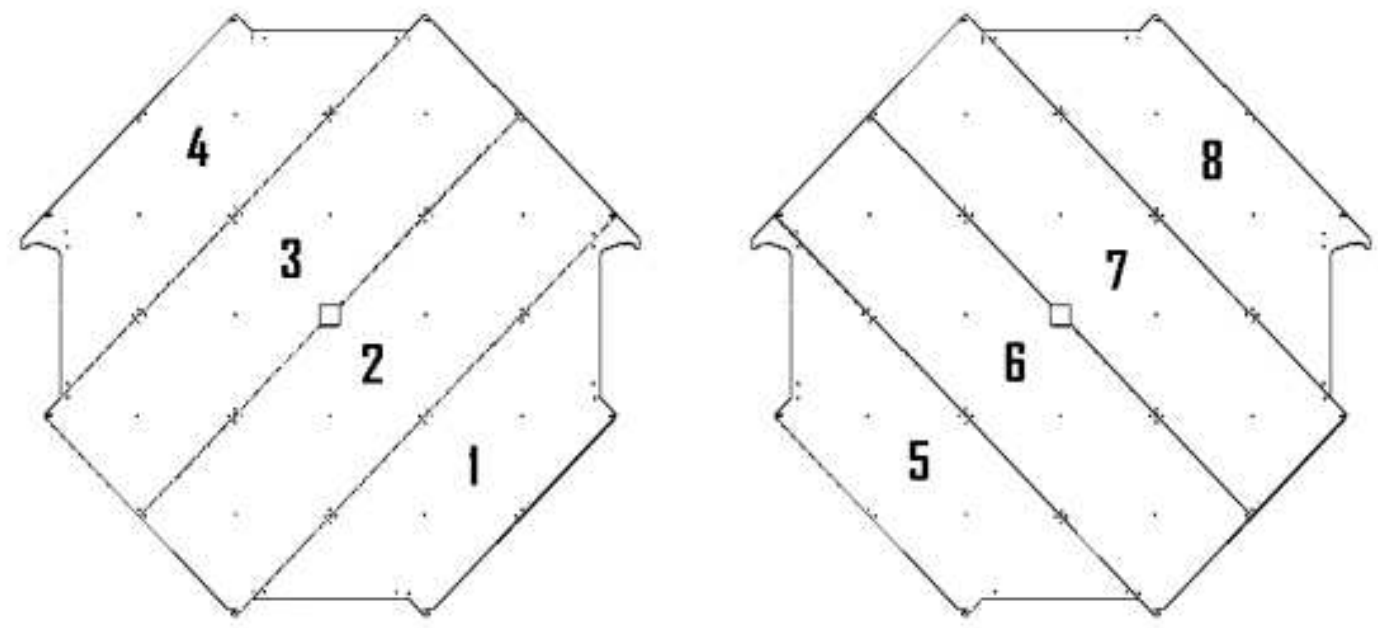

Figure 3.7: The steel for the MINOS far detector is divided into $2^{*} 4$ sections, where the both of the four section groups that create the detector octagon are welded together in a $90^{\circ}$ alignment to form the full octagon [69].

front of the previous steel plane. The plane is physically supported by the "steel ears" that rest on a rail, and by eight bolts (one at each octagonal edge corner) that attach to the previous plane. Each plane is $2.54 \mathrm{~cm}$ thick, and separated from its adjacent planes by $3.5 \mathrm{~cm}$. The first and second supermodule consists 249 and 237 steel planes respectively.

\section{- Magnetic Field -}

The hole in the middle of each steel plane is left for the passage of a coil that will generate a magnetic field to bend the path of muons. A $450 \mathrm{~V}$ high voltage power supply generates an 80 A current for each magnet coil. Control software ramps the power up and down and monitors the voltage and current at the supply. Thermocouples in several locations are also monitored by the controls for the coil temperature. The coil is water cooled, but if the temperature reaches to high the coil current is automatically tripped off. The coil current is read to a database that is used by data quality processes to determine the usability of a detector data. 


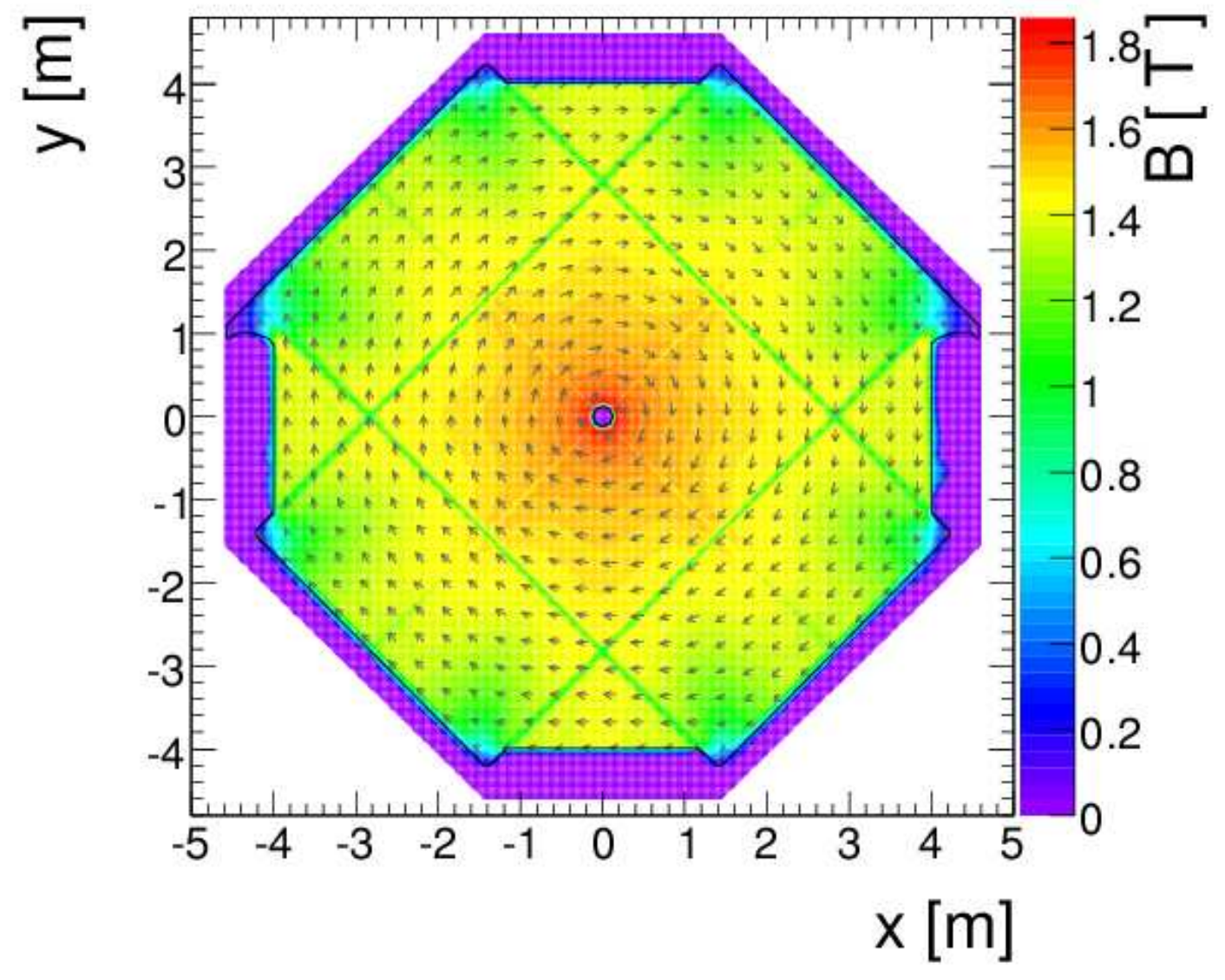

Figure 3.8: Magnetic field map for the MINOS far detector

\section{- Scintillator and Fiber -}

One side of each plane of steel is covered with strips of plastic scintillator that will emit blue light when a charged particle passes through. The extruded polystyrene scintillator strips are $1 \mathrm{~cm} \mathrm{x} 4 \mathrm{~cm}$, and of varying length from $8 \mathrm{~m}$ to $4 \mathrm{~m}$. The polystyrene scintillator contains easily excitable aromatic molecules to induce the scintillation of optical photon. This is achieved with the doping of primary (1\% PPO) and secondary $(0.03 \%$ POPOP $)$ flours that absorb the photons at a fast rate and re-radiate them at a displaced wavelength where the scintillator is less likely to capture the photons. The strips are also lined with a reflective polystyrene coating mixed with $15 \%$ Ti $\mathrm{O}_{2}$ to 
maximize the light yield.

All but two of the steel planes are covered with 192 scintillator strips that are oriented at a $45^{\circ}$ angle to the vertical. Strips in alternating planes views are situated at perpendicular angles to enable 3D event reconstruction. The scintillator strips are collected groups of either 20 or 28 , and assembled inside of an aluminum housing called a detector scintillator module. Two types of modules (types A and B) contain 28 strips, and are situated on the outer edges of the steel plate. The two other module types contain twenty strips, and serve the inner portion of the steel plate.

(a) U-View Scintillator Plane

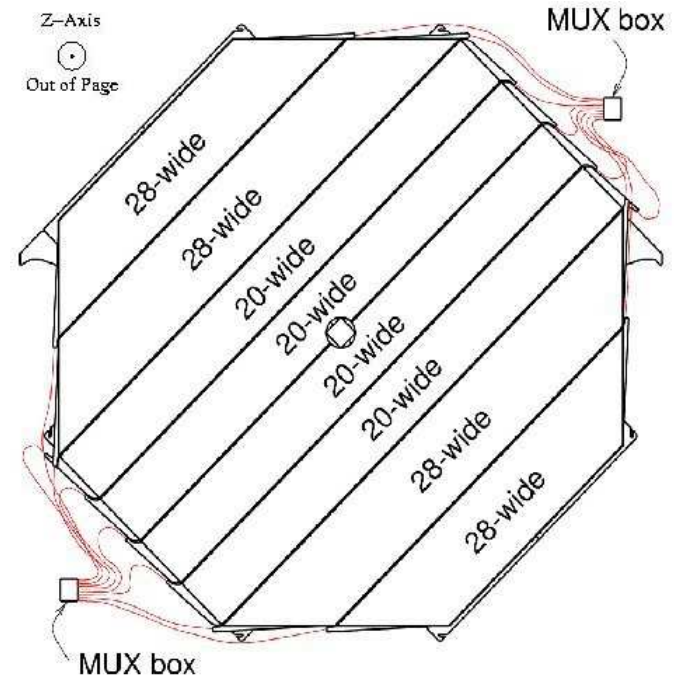

(b) V-View Scintillator Plane

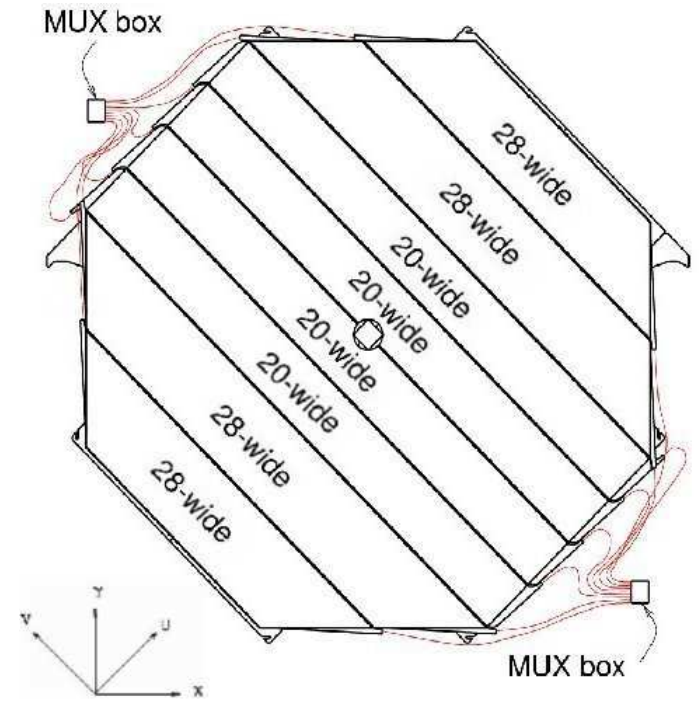

Figure 3.9: The layout of the scintillator modules on the steel plane as viewed looking southward

The scintillated photons are collected by $1.2 \mathrm{~mm}$ diameter wavelength shifting (WLS) fibers that are glued into grooves cut in the center of the $4 \mathrm{~cm}$ wide strip edge that runs the entire strip length. The Kuraray Y-11 WLS fibers absorb are most efficient are absorbing blue photons, with peak absorption at $420 \mathrm{~nm}$. Light is then re-emitted in the green part of the spectrum, with peak emission at $520 \mathrm{~nm}$ [64]. The reemission of light from the WLS fibers occurs with a characteristic decay time of approximately 8 ns 64.

The WLS fiber in each strip tails beyond the strip end into a plastic manifold and to an optical connector located at each end of the scintillator module. The optical 
(a) A-Type Module Connector Manifold

(b) C-Type Module Connector Manifold
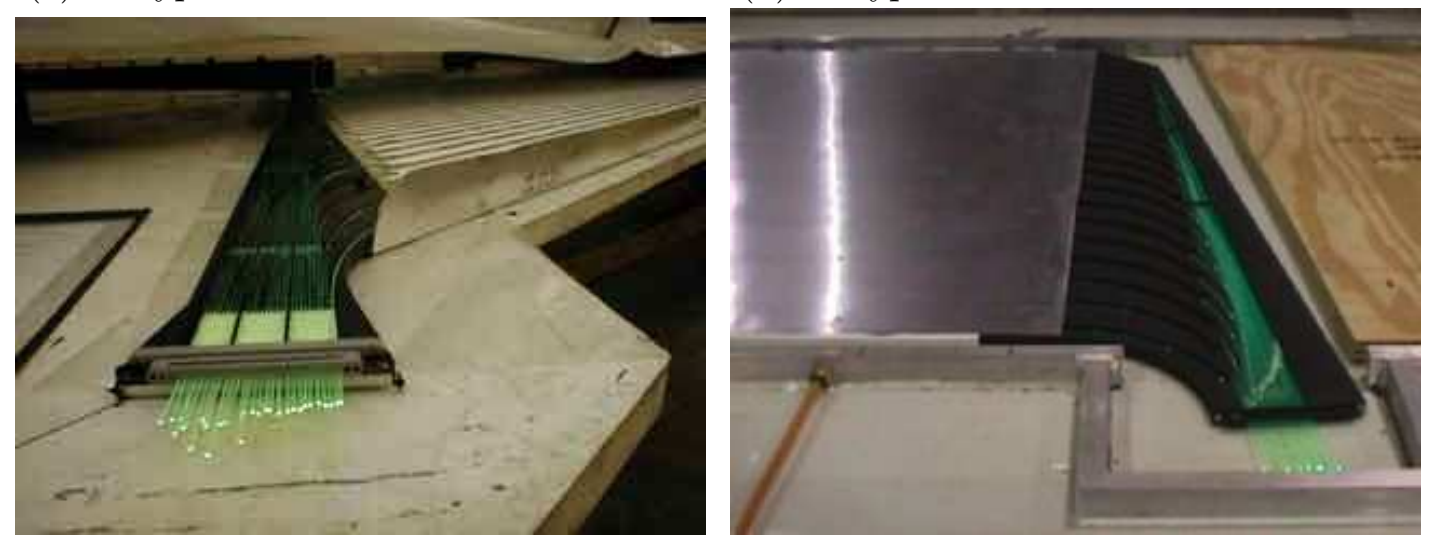

Figure 3.10: Extension manifold on a 20 strips scintillator module [70].

connectors are attached to multi-pixel photo-multiplier tubes by clear polystyrene fibers. Light attenuation along WLS and clear fibers has been measured during construction and was tested in a four plane prototype build at Fermilab [70]. The fiber attenuation and time response is used in the detector calibration to reconstruct the energy deposition and time of the particle traversing the scintillator strip.

\section{- Cosmic Ray Veto Shield -}

To select atmospheric neutrino events the high background of cosmic-ray muons must be reduced. The veto shield is the collection of strips above the detector that are used to tag cosmic-ray muons entering through the top or sides of the detector. To this end, a series of the 20-strip scintillator modules have been mounted above and on the sides the far detector to detect cosmic rays as they enter the detector [71].

The modules are placed along the top and sides of the detector to provide a hermetic tagging mechanism for downward-going muons entering the detector. The structure is supported by a metal framework as well as by the detector itself. The shield consists of four overlapping sections of scintillator strips that are aligned to the Z-axis, where two section are above each supermodule. The top section shown in Figure [3.11]a is double layered, while the upper and lower sides are single layer. The double layering of the top section is intended to increase the tagging efficiency in the direction of the maximum cosmic-ray muon flux. Figure 3.11] b shows an example of a cosmic muon event tagged 
by the veto shield.

(a) Veto Shield Design

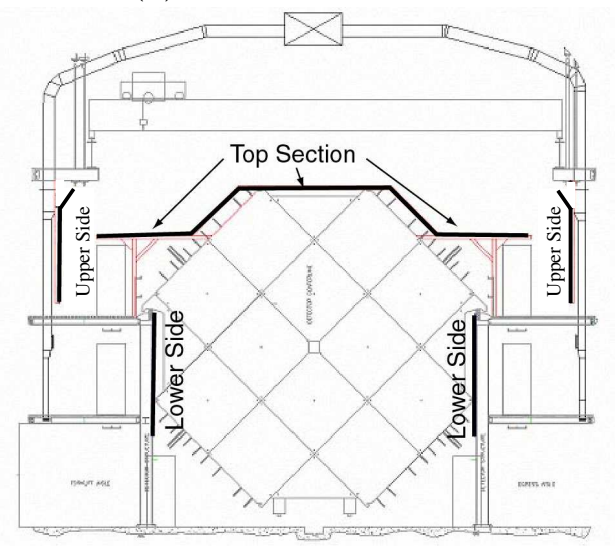

(b) Event Tagged by Veto Shield

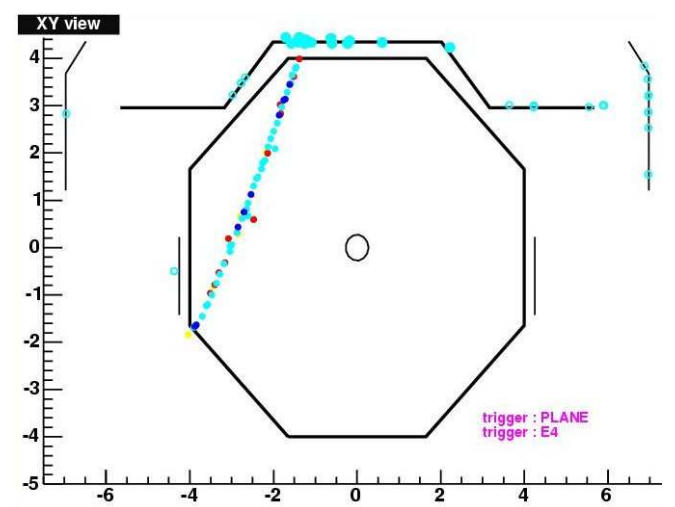

Figure 3.11: (a) Design concept for the MINOS far detector cosmic-ray veto shield [71] and (b) example event tagged by the cosmic-ray veto shield.

Groups of 8 fibers run from both ends of the scintillator strips are ganged to a PMT pixel on each end, such that the multiplexing of veto shield fibers is identical on both sides of the shield module. A group of eight scintillator strips attached to a single PMT pixel cannot be demultiplexed, and is called a shield plank. The spatial resolution of each plank is $10 \mathrm{~cm}$ in the $\mathrm{X}-\mathrm{Y}$ plane and $8 \mathrm{~m}$ in the $\mathrm{Z}$ plane. The tagging procedure and efficiency is discussed in depth in Section [7.6

\section{- Photon Measurement and Optical Summing -}

The clear fiber cables from the each scintillator module are attached to a $M U X B o x$ (Figure $3.12 \mathrm{a}$ ), that contains batches of clear fibers that are attached to M16 Hamamatsu photo-multiplier tubes (Figure 3.12,b) bundled in groups of eight. [72] The fibers that are grouped by eight on a single PMT pixel are chosen to be able to most easily resolve the fiber hit ambiguity with the double- ended nature of the readout. The grouping on the east end of the fibers is different from the west end to make this resolution possible. The demultiplexing procedure and performance are discussed in Section 5.3

The M16 PMTs convert the light from the scintillator into an electronic signal of photo-electrons to be readout by the electronics. The PMT quantum efficiency for photon conversion of WLS peak emission photons is approximately 13\%. A muon 
(a) MUX Box

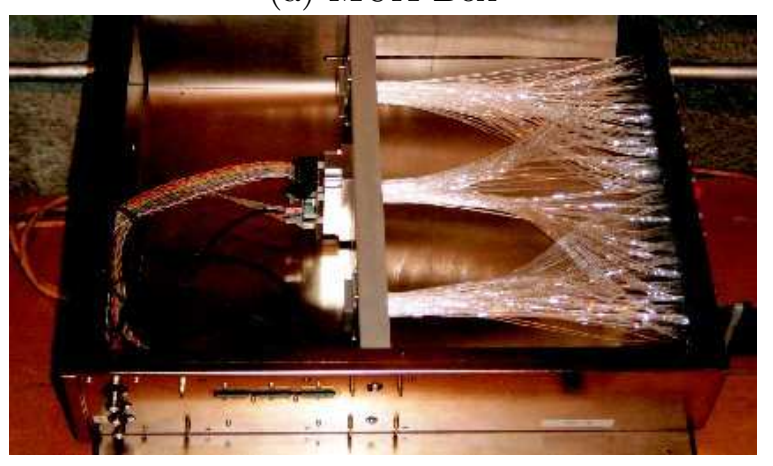

(b) M16 PMT

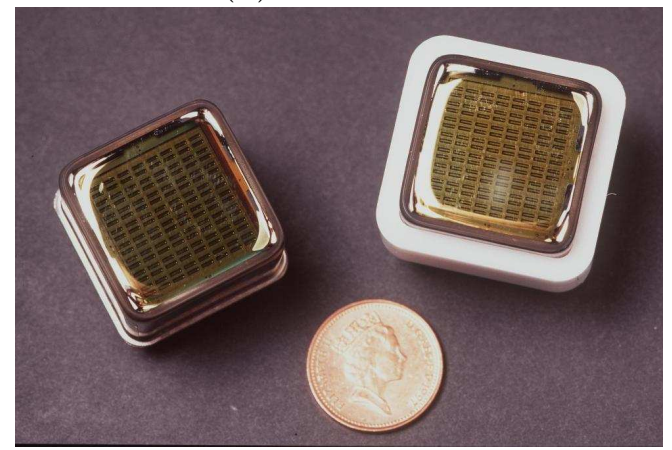

Figure 3.12: (a) Fiber multiplexing box and (b) 16 pixel photo-multiplier tubes (M16 PMT). Each MUX Box contains 3 M16 PMTs [72.

that deposits the minimum-ionizing energy in a scintillator strip will typically readout 5 photoelectrons at each strip end. The PMTs operational gain is typically $10^{6}$, producing photo-anode signals $\sim 1 \mathrm{pC}$. Studies have shown that the integrated charge on the photoanode is linear with the input signal on the photo-cathode within $5 \%$ for signals up to $100 \mathrm{PE}$ [2]. The readout of each PMT is triggered by a common dynode signal, for which the trigger threshold is set to $\frac{1}{3} \mathrm{PE}$.

The rate of dynode triggers caused by background noise in the detector has been measured to be $5-10 \mathrm{kHz}$ for each PMT This has been diagnosed as the result three effects. Two anticipated causes of background dynode triggers are natural radioactivity in the detector hall and PMT dark counts. The natural radioactivity in the mine was measured by the Soudan2 experiment during their construction phase. The natural abundance of radioactive elements the rock $\left({ }^{238} \mathrm{U},{ }^{232} \mathrm{Th},{ }^{40} \mathrm{~K}\right.$ e.g. $)$ produce a continual flux of radiation on the detector surface. The decay chains also can produce ${ }^{222} R n$ gas, which is in a gaseous form and can penetrate deep into the detector. Thermionic emission of electrons from the photo-cathode are the source of PMT dark counts. The combination of these two background effects is expected to produce $1.2 \mathrm{kHz}$ (natural radioactivity $)+0.5 \mathrm{kHz}(\mathrm{PMT}$ dark counts $)=1.7 \mathrm{kHz}$, which is less than the measured noise rate in the detector. The remaining noise is caused by photon emissions originating in the WLS fiber which result in single photo-electron signals. These anomalous photon emissions are believed to be the result of long-term relaxation of mechanical stress caused by the glueing of WLS fibers into the scintillator strips during detector construction [73. 


\section{- Electronics, Trigger and Data Acquisition -}

The signal of photoelectrons on each PMT photo-anode is read out with a customized version of the IDE AS Viking VA chip, an integrated circuit that contains multiple channels of shaping amplifiers and sample and hold circuitry [74, 75]. Each VA chip reads out the 16 pixels on a single PMT with 22 VA readout channels (16 pixel readout channels for all PMT photo-anodes, one channel for light injection PIN diodes, and five channels to correct common mode fluctuations).

The VA chips are grouped by three onto VA Front - End Boards (VFBs) attached to the side of MUX boxes. Two VFBs are attached to a Varc Mezzanine Module (VMM) that reads the PMT channel signals, and a Event Time Controller (ETC) that controls the sample and hold circuitry in the VA chip. Each ETC-VMM pair reads and controls two VFBs, with six total VA chips. Up to six ETC-VMM chips are housed on a $V A$ Readout Control card (VARC). Three VARCs are stored in a single VME crate, and $8 \mathrm{VME}$ crates service each side of the detector.

The common dynode signals from each PMT are monitored by the VFB. When a dynode signal is detected a signal is sent to the $V A R C$, where the coincidence trigger is implemented. The coincidence trigger requires at least 2 out of $36 \mathrm{VA}$ chips on a single VARC to trigger within $400 \mathrm{~ns}$ in order to activate the readout. The ETC timestamps the triggered event, and directs the VA chips to read out the PMTs that have triggered, at which point each VA channel is digitized by the VMM. Further dynode signals from the PMTs are ignored in this time, incurring a short dead time. The VA readout and digitization operates at $200 \mathrm{kHz}$ per chip, allowing a $5 \mu \mathrm{s}$ dead time in each VA chip. If all six chips that a $V M M$ services are readout, up to $30 \mu s$ dead times can occur.

The shield PMTs are also read out by VA chips and digitized by $V M M s$ which share common $V A R C s$ with the detector readout. The trigger conditions are set differently for the shield readout in order to optimize the muon tagging efficiency. To reduce tagging inefficiencies that arise from single $\mathrm{PE}$ noise, the dynode threshold is set to a level equivalent to 1-2 PE. The shield readout is also not included in the $V A R C$ trigger so that all shield activity is recorded.

A 14-bit ADC is used to digitize the PMT signals into, where the range has been set to approximately $70 \mathrm{ADCs}$ per $\mathrm{PE}$, giving a maximum signal of $2^{14} / 70 \sim 250 \mathrm{PE}$. The digitized signals are hand off to a sparsified chip that subtracts pedestal thresholds 
from each signal and removes all signals below 20 ADC. The data is transferred out of the VARC with a dual buffer systems that allows the simultaneous collection of data and readout of data. The data is readout from the VME crate by a single-board Readout Processor (ROP) that accumulate the data into second-long timeframes. The time frames are fed through Branch Readout Processors (BRPs) to one of several Trigger Processors (TPs) running on a PC farm.

The TPs implement a set of trigger algorithms that search for spatial and temporal clusters in each group. If a group of hits satisfies one or more of these trigger conditions it is passed to the output stream of the TPs. The trigger algorithm implemented at the Far Detector for normal data collection is is a $4 / 5$ plane trigger algorithm that requires 4 out of 5 contiguous planes in the detector to register hits. Events that pass this final trigger condition are written out to data files. The data collection is segmented in 8 or 24 hours runs, which further divided in to hour long subruns. More detailed information regarding the data acquisition (DAQ) can be found in references [76, 68] 


\section{Chapter 4}

\section{Monte Carlo}

When an event is triggered in the far detector there are three question that must be answered in order to use that event trigger analytically: (1) What does the response of the electronics say about how particle moved through the detector? (2) What does the movement of the particle through the detector say about the particle (particle type, energy, direction, etc.)? (3) What does the information about the particle say about the flux of that particle type at large? In order to answer these questions, the theoretical and experiment knowledge of the detector technology, particle interactions, and particle flux are used to simulate large samples of "Monte Carlo" events to compare to data events. The term Monte Carlo was coined by mathematician Stanislaw Ulam in reference to the famous casino district in the French Riviera where the fictional character James Bond would frequent for games of Baccarat [77]. A Monte Carlo method uses statistical sampling from probability distributions to simulate the gross behavior of an unknown parameter [8].

In this chapter, the simulation of far detector events is presented in three sections that are ordered reverse to the three questions asked above: (1) incident particle flux, from generation point to the detector, (2) interaction of the particle with the detector material, and (3) response of the active detector elements to the traversal of charged particles through the scintillator strips. 


\subsection{Incident Particle Flux}

To study particles in the detector, flux models must first be generated that illustrate the rate of incident particles as a function of energy, direction, and in some cases time.

\subsubsection{Atmospheric Neutrinos}

In order to perform atmospheric neutrino studies, the flux of neutrinos at a detector site must be predicted as accurately as possible. This prediction is performed by simulating cosmic-rays hitting the upper atmosphere, making hadrons, and having the hadrons decay to neutrinos. Oscillation studies rely on the comparison of the observed flux to the expected flux.

The flux model developed by Barr et al [2] was used for this thesis to measure the atmospheric neutrino double ratio. The flux model developed by Battistoni et al [38. was also used in the exploration of systematic variances. The Bartol model simulates the atmospheric neutrino flux at the MINOS far detector by performing a full threedimensional treatment of cosmic-ray interactions in the atmosphere. Cosmic rays that are incident on the top of the atmosphere all over the whole globe produce secondary particles that are tracked through the atmosphere.

The primary flux of cosmic-ray is comprised of proton $(\sim 75 \%)$ and heavier ionized nuclei $(\sim 25 \%)$ parameterized by an energy power law for each incident nuclei. The primary flux is modulated by the solar wind, which ebb and flows in an 11-year cycle. When the solar wind is stronger, the lower energy portion of the primary cosmic-ray flux is suppressed and the neutrino flux is reduced. This thesis takes place near the maximum end of the solar cycle, and thus the simulated set used for analysis assume the solar maximum.

The Bartol model also accounts for variations in the Earth's magnetic field over the globe. Earth's magnetic field acts as a filter for low-energy cosmic rays incident on the atmosphere. Cosmic rays with high enough energy will penetrate through the magnetic field and interact with the atmosphere, while cosmic rays with lower energy are deflected back out to space. The cut-off energy depends on the magnetic field intensity and the angle at which cosmic rays approach the Earth. Cosmic-ray with a charge $>+1$ will be more easily deflected by the Earth's magnetic field. 
The cosmic rays that manage to interact with the atmosphere will produce a hadronic cascade. Any particle that would arrive at the Earth's surface before decaying is not considered for neutrino production. For particles that may decay in the atmosphere, decay and interaction are still competing processes. A decay and interaction length is chosen randomly from a probability distribution function, and these lengths are compared to determine whether the particle decays or interacts.
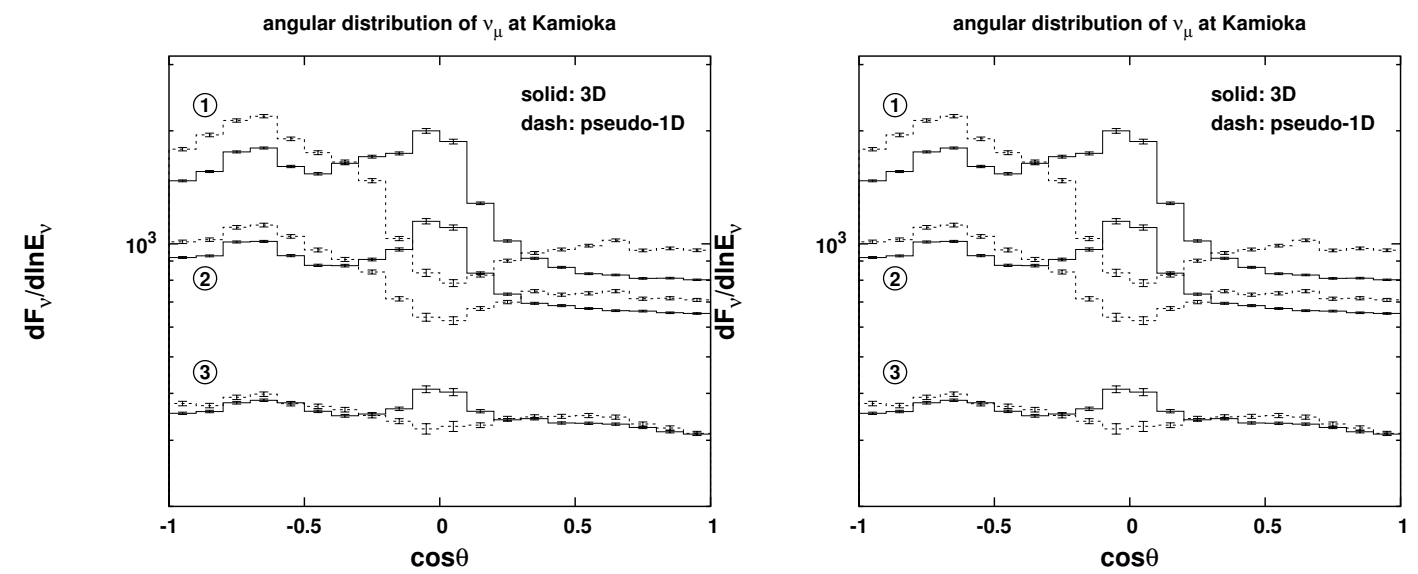

Figure 4.1: Zenith angle distribution of atmospheric $\nu_{\mu}$ flux for a range of energies (solid lines $=3 \mathrm{D}$ model, dashed lines $=1 \mathrm{D}$ model) as predicted by Barr et al. [2]. Left side is $\mathrm{Sub}-\mathrm{GeV}$ neutrino and right side is multi-GeV neutrinos.

Figure 4.1 shows the predicted zenith angle spectra of the atmospheric neutrino flux from the Bartol model for a range of neutrino energies for both 1D and 3D models. The 1D model only handles the hadronic shower in the direction of the detector, while the 3D model takes the computationally slower approach of handling the entire hadronic shower. The 3D model presents significant flux differences near the horizon for lower energy neutrino. The neutrino flux declines as the neutrino energy increases. The updown asymmetry, apparent by the higher flux for $\cos \theta_{z}>0$ also declines as the neutrino energy increases.

The modelling uncertainties in the atmospheric neutrino flux are dominated by the uncertainties in the primary cosmic-ray flux and hadron-production models [6]. Figure 4.2 a shows the uncertainty of the overall flux is found to be $15 \%$. The uncertainty of the ratio of $\nu_{\mu} / \nu_{e}$ is shown in figure 4.2. b. This flavor ratio of special interest to the analysis performed in this thesis, and the measurement of the double flavor ratio. 

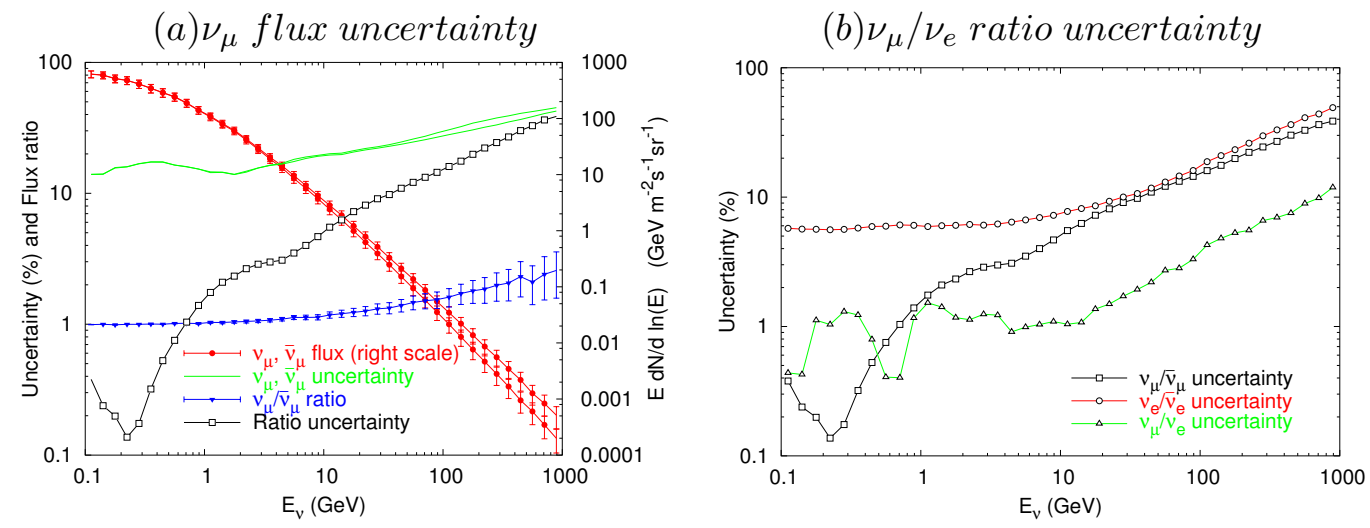

Figure 4.2: Atmospheric neutrino flux uncertainty for the Bartol model [6] .

\subsubsection{Cosmic-Ray Muons}

Atmospheric neutrinos experiments are always deep underground to shield the experiment from the intense cosmic-ray muon flux at the Earth's surface. Even deep underground, atmospheric neutrino detectors still have to contend with the background from muons that have high enough energy to penetrate the rock above the detector hall. These cosmic-ray muons must be simulated to understand this background, and what event selection procedure must be implemented to remove these events.

To obtain a flux of cosmic rays incident on the MINOS Far Detector, a simulation process follows particle fluxes starting in the upper atmosphere. The particles are propagated to the Earth's surface, and parameterized by energy and direction as shown in Equation 4.1 [79]. This parameterization is valid for muons energetic enough to penetrate the Earth's crust before decaying $\left(E_{\mu}>100 \mathrm{GeV}\right)$.

$$
N(E, \cos \theta)=\frac{0.14 \cdot E^{-2.7}}{c m^{2} \operatorname{srGeVs}}\left(\frac{1}{1+\frac{1.1 E \cos \theta}{115 G e V}}+\frac{0.054}{1+\frac{1.1 E \cos \theta}{850 G e V}}\right)
$$

The flux of $\mu^{+}$and $\mu^{-}$are generated with a ratio of $\mu^{+} / \mu^{-}=1.25$, where the asymmetry is due to the excess of positively charge hadrons produced by the primarily positively charged primary flux. The flux of cosmic muons at the far detector is calculated by propagating the cosmic muon flux measured at the surface through a digitized map of the rock overburden above the Soudan cavern [80]. The rock above the Far 
Detector cavern is primarily "Lake Vermillion Greenstone", with a measured density of $2.8 \mathrm{~g} \mathrm{~cm}^{-3}[80$. This rock is interspersed with iron ore pockets, causing the average rock density to vary with direction. The far detector cosmic muon simulation uses the rock density map calculated by the Soudan 2 experiment with cosmic-muon data.

$$
\begin{aligned}
& \frac{d E}{d x}=A+\frac{E}{L} \\
& E_{X}=\left(E_{0}+A L\right) e^{-X / L}-A L
\end{aligned}
$$

Muon energy loss through the rock map is parameterized as shown in Equation

4.2 The term $A \approx 1.9 \mathrm{MeV} / \mathrm{g} \mathrm{cm}^{-2}$ is from ionization energy loss. The second term in Equation 4.2 represents radiative energy loss, where $L \approx 2.5 \times 10^{5} \mathrm{~g} \mathrm{~cm}^{-2}$. In practice $A$ and $L$ both vary with energy and $\frac{d E}{d x}$ fluctuates stochastically, all three terms are nonetheless treated approximately as constant. With the approximation in the Equation 4.2 the energy as a function of depth $E(X)$ and surface energy $E_{0}$ is calculated by integration and expressed in Equation 4.3. The final flux at the detector is found by combining the surface flux in Equation 4.1 with the energy loss formula in Equation 4.3 and the rock map measure by Soudan 2 .

\subsection{Particle Interactions with Detector Material}

Given a flux of particles incident on the detector, these particles must next be propagated through the detector. When propagating a particle through a material, care must be taken to properly track the parent particle and all daughter particles that result from interaction of the parent particle with the detector material. Energy loss by any particle passing by nuclei must be found statistically across a length of many nuclei based on the overall material property.

- Simulation of Neutrino / Nucleon Interactions - The physics of neutrino interactions with nucleon were introduced in section 2.2. To simulate the interaction of a neutrino with a nucleon the Neugen 3 simulation package [81] was employed.

- Simulation of Lepton / Nucleon Interactions - Charged leptons (electrons, muons, and taus) passing through a material lose energy to that material as a 
result of electromagnetic exchanges with nucleons in the material. The Geant 82] program handles the propagation of charged particles through the detector material, and the GCALOR [83] program models the hadronic interactions. The GHEISHA [84] program was also used as a systematic variance of the hadronic interaction model.

- Propagating Charged Particles Through a Magnetic Field The magnetic field in the far detector was introduces in section 3.2.2. A charged particle passing through the field generated by this coil will be bent according to the particles velocity and mass according to the Maxwell's equations. The Geant3 [82] program uses magnetic field maps to bend charged particles as they pass through the iron plates.

The Neugen3 package [81] models interactions between neutrinos and matter. The matter may be regarded as bound nucleons inside of nuclei or quark-parton functions inside of nucleons. For an incident neutrino, Neugen3 uses a Monte Carlo sampling technique to determines whether an interaction will take place. The Neugen 3 program then selects one of three types of interactions: quasi-elastic scattering (QE or QES) which is dominant below $1 \mathrm{GeV}$, resonances (RES) in the energy range $1-3 \mathrm{GeV}$, and deep-inelastic scattering (DIS) for energies above $3 \mathrm{GeV}$. Each interaction cross-section is tuned to fit the available experimental data.

The incident neutrino scatters off a nucleon inside of a nuclei for QES and RES interactions. The bound nucleons inside of the nucleus are treated as a Fermi gas, where each nucleon possesses a Fermi momentum of $\simeq 230 \mathrm{MeV}$ and a binding energy of $\simeq 30 \mathrm{MeV}$. In quasi-elastic scattering processes, the neutrino scatters elastically off the nucleon. In resonance scattering processes, the neutrino interacts to form a resonance (dominantly $\Delta(1232)$ which then decays into a final state usually containing one or more pions.

The incident neutrino scatters off of a quark-parton distribution function inside of a nucleon in the case of DIS interactions. The structure functions, which are calculated from the parton distribution functions, are used to express the interaction cross-section for the DIS process. After an neutrino interaction has taken place, the resulting hadrons exiting the nucleus may be absorbed or re-scattered. The intra-nuclear effects of these 
hadrons are simulated by the Intranuke package [85].

\subsection{Active Detector Simulation}

The active detector is defined as the portions of a particle detector which attempt to make direct physical measurements of particle attributes. This is opposed to the passive portions of a detector, which measure physical aspects of particles indirectly by affecting their motion. The active detector is simulated by the PhotonTransport and DetSim packages [86].

\subsubsection{Scintillator Strips and WLS Fiber}

The process of a charged particle passing through the scintillator strips and depositing energy is modelled by the PhotonTransport package [86]. As the name implies, this package simulates photons from their point of creation in the scintillator all the way to their subsequent conversion to photo-electrons on the PMT cathode. The gminos process that propagates each charge particle through the detector records each energy deposition in a scintillator strip. For each scintillator energy deposition, PhotonTransport generates a number of blue photons according to Birks' Law as expressed in Equation 4.4 87. where: $d E$ is the scintillator energy deposition, $L_{0}$ is the scintillator light output, $C_{0}$ is the strip response correction, $C$ is normalization term for tuning purposes, and $k_{B}$ is birk's constant.

$$
N_{\text {blue } \gamma}=C \frac{L_{0}}{C_{0}} \frac{d E}{1+k_{B} \frac{d E}{d x}}
$$

A fraction of the blue photons are captured in the WLS fibers, where they are converted to green photons. The capture and conversion efficiency of blue scintillator photon into green WLS photons is determined using a set of probability distribution functions (PDFs) relating the positions and times of green photons to the positions and times of the blue photons. These PDFs are constructed with detailed Monte Carlo

simulations of photon emission, propagation and absorption in the scintillator strips and capture efficiency in the WLS fibers. The green photons are tracked and attenuated 
individually down the WLS and clear fibers. The photons are converted into photoelectrons at the PMT photo-cathodes using a flat probability of $13 \%$, equal to the measured quantum efficiency of the M16 PMTs. Single dark-current photo-electrons are also generated to simulate background noise in the detector.

\subsubsection{Photo-multiplier Tubes and Electronics Response}

The simulation of the photomultiplier tubes The DetSim package [86] models the amplification of photoelectrons (PEs) through the M16 PMTs. The final signal from the PMT is then passed through a model for the digitization of charge by the VA electronics. Finally, DetSim implements the DAQ trigger from the digitized VA signal. The PhotonTransport simulation found the total number of PEs on a photo-cathode. First, the PE signal is amplified through the first pair of dynodes, and the resulting charge is smeared to account for fluctuations due to secondary dynode emissions. The charge on the photo-anode is then calculated with the PMT response curve.

After simulating the amplification of the PE signal through the PMT, DetSim simulates the VA readout electronics response. Signals satisfying the 2/36 VARC level trigger are amplified according to VA response curves and smeared according to pedestal widths. The signals are then digitized, and hits with $<20$ ADCs are removed. The 4/5 plane DAQ level trigger is applied to the hits to select interesting physics events. These events are written out similarly to real data, so that they may be compared to the data set. In order to compare data and simulated events, a reconstruction process is outlined in the next chapter. 


\section{Chapter 5}

\section{Event Reconstruction}

Chapter 4 introduced the three important questions to ask about an event trigger. These questions are: (1) What does the response of the electronics say about how the particle moved through the detector? (2) What does the particle's movement through the detector say about the particle (particle type, energy, direction, etc.)? (3) What does the information about the particle say about the flux of that particle type at large? The event reconstruction process answers the first two of these questions by implementing the following steps:

1. Noise Filter - Remove those events that fail the thresholds of both minimum and maximum detector activity.

2. Digit Reconstruction - Determine the strip energy and timing from a calibrated PMT and electronics response.

3. Demultiplexing - Resolve the ambiguity caused by the optical summing of the strips.

4. Track and Shower Finding - Use clustering techniques to accumulate hits into tracks and showers.

5. Direction and Energy Resolution - Determine the direction and energy of tracks and showers based on the energy deposition in steel and curvature of path through the magnetic field. 
The MINOS experiment uses a job control framework, where a set of information is passed progressively from one analysis method to another. This job control framework implements the reconstruction chain above by passing a set of information to a progression of $\mathrm{C}++$ classes. Each analysis method modifies and adds to the set of information that job control holds, such that each analysis method will have access to information from all previous analysis methods.

\begin{tabular}{|llr|}
\hline FarDetDataQualityModule :: Reco & - & \\
\hline DigitListModule :: Get & - & \\
DigitListModule :: Reco & - & Doise Filter \\
FilterDigitListModule :: Reco & - & \\
FarDetShieldPlankListModule $::$ Reco & - & \\
\hline DeMuxDigitListModule $::$ Reco & - & \\
\hline FarDetStripListModule $::$ Reco & - & \\
FarDetSliceListModule $::$ Reco & - & Track and Shower Finding \\
AtNuFindModule :: Reco & - & \\
\hline AtNuFitModule $::$ Reco & - & Direction and Energy Resolution \\
FarDetEventModule :: Reco & - & Write Analysis File \\
\hline NtpMaker :: Reco & - & \\
\hline
\end{tabular}

Table 5.1: Reconstruction chain as viewed by the job control framework.

The ensemble of event reconstruction software was designed and coded by Andrew Blake, Caius Howcroft, Mark Thomson, and other MINOS collaborators at Cambridge University. This event reconstruction chain is referred to within the MINOS collaboration as the "Cambridge Reconstruction", as opposed to the "Standard Reconstruction". The standard reconstruction uses demultiplexing and track and shower finding algorithms that are optimized for the identification of beam neutrino interactions. The Cambridge reconstruction algorithms are instead optimized for the identification of atmospheric neutrino interactions. The Cambridge group is responsible for the bulk of the work explored in this chapter, and is owed an immense debt of gratitude by the author. 


\subsection{Noise Filter and Digit Reconstruction}

The plane trigger described in section 3.2.2 requires that at least 4 out of 5 planes have activity in order to write an event out to a data file. The sensitivity of the trigger is optimized to maximize the retention of potentially interesting events while minimizing the size of output. Thanks to modern computing technology, the output minimization was not weighted heavily in the trigger design. As a result of the trigger sensitivity, a large number of these triggered events will still fall below a threshold of what could be considered an interesting event.

The noise filter is the first stage of the reconstruction, and is implemented by the method FarDetDataQualityModule :: Reco [88]. The noise filter deselects triggered events based on three criteria: a minimum and maximum number of active channels associated with a supermodule plane (not including shield channels), a maximum number of VA chips (plane or shield chips) with a low singles rate, and a maximum number of active channels associated with the light injection box that is pulsing, (only plane channels, as shield channels are not pulsed).

There are 23232 plane channels in the far detector ([24 8-strip bunches / plane] * [2 channels / 8-strip bunches] * [484 planes $]$ ). Each triggered event will contain at least 4 active plane channels, by requirement of the plane trigger. The noise filter additionally requires that there be more than 10 active channels and no more the 1000 active channels. With these plane channels, there are 2904 VA chips ([6 chips / plane] * [484 planes]) associated with the supermodule planes, and 64 VA chips ([16 chips / shield section] $*$ [4 shield sections]) associated with the veto shield, for a total of 2968 VA chips in the far detector. The data stream from the far detector contains detector summary information along with the detector trigger events. The FarDetDataQuality package uses the detector summary information to flag each chip in one of four states:

- Cold Chip - A chip that has a singles rate less than $50 \mathrm{~Hz}$ is flagged a "cold chip".

- Hot Chip - A chip with a singles rate greater than $2500 \mathrm{~Hz}$ is flagged a "hot chip".

- Busy Chip - A chip with activity in the triggered event that is more than 150 
ns before the trigger time is flagged a "busy chip".

- Good Chip - A chip that is none of these is flagged a "good chip".

VA chips that have been flagged as cold are considered unusable in an event. If there are more than 50 cold plane or shield chips, the event is discarded by the noise filter. The chip flags are stored for use in later analysis.

The final check assures that a light injection pulsing event did not enter the data stream. The detector summary information identifies which light-injection pulser box is operating at any given time. Each pulser box pulses the strips that are attached to a single VA crate on each side of the detector. Each pulser box thus maps to 3072 channels. If more than 500 of these pulsed channels are active then the trigger is discarded. The purpose of the reconstruction noise filter stage is to reduce the number of uninteresting events that will be handed to the next reconstruction stages. The raw data event rate is reduced by a factor of $\sim 1 / 10$ by the noise filter.

\subsection{Digit Reconstruction}

The low-level readout of the detector is bundled by individual channels into objects from the C++ class RawDigit. Each RawDigit contains the digitized quantities of the timing and pulse height response of the PMT. Each VA channel corresponds to a PMT pixel, with 8 attached fibers. In order to compare digits to each other for the later stages of the reconstruction, the response of the electronics channels have been calibrated to measure the energy and timing of each hit. Accurate calibration of the energy response is important in the measurement of shower energies and accurate calibration of the timing response is important for measuring the track direction. Both are critical for atmospheric neutrino oscillation analyses. The MINOS detector calibration scheme has been discussed extensively [89, 90, 91], and will only be outlined briefly here.

\subsubsection{Energy Response Calibration}

To calibrate the energy response of each channel, the detector utilizes two calibration systems: light injection [92, and charge injection [74]. The ADC readout of each channel is calibrated by the charge injection system. Each channel has a known amount 
of charge injected at the PMT base, and the response is measured by the DAQ. The result is that small variations between each channel can be normalized such that the ADC count from each channel are consistent within $1 \%$.

The next step in calibration is to convert the ADC counts from each channel to a photo-electron (PE) count from the PMT. This conversion is treated differently for low ADC count, where the response is linear, and high ADC count where the PMT signal saturates and the response in no longer linear. The light injection system (Figure $5.1 \mathrm{a}$ ) uses blue LEDs to pulse light of a given intensity directly on the WLS fibers to mimic scintillator light. The pulser LEDs are housed in pulser boxes that contain 20 LEDs each and a optical fanout that distributes the LED light to 70 fibers. Two of those fanned fibers are also directed to two separate PIN diode photo detectors that measure the LED light output: a high-gain detector to measure low-light signals and a low-gain detector to measure high-light signals. The pulser box is also connected to a trigger PMT, that identifies in the data stream when a pulser box is active.

(a) LI Schematic View

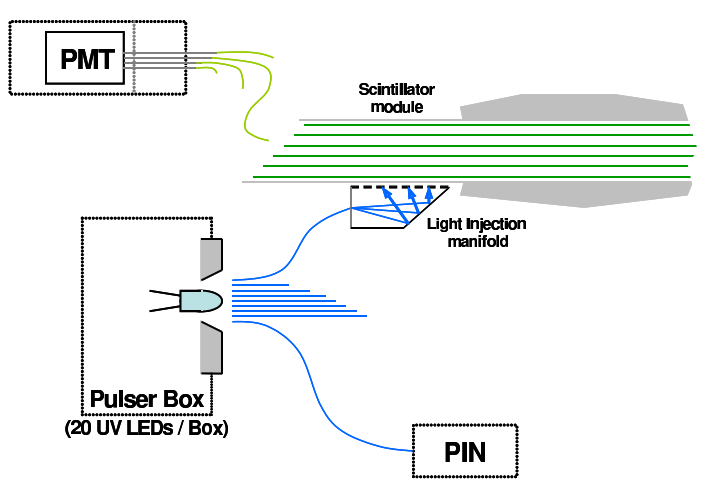

(b) LI Response

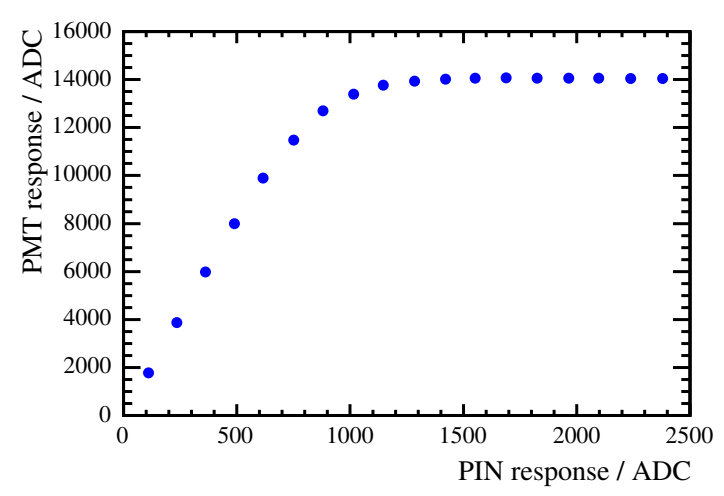

Figure 5.1: (a) Schematic view of the MINOS "Light Injection" system. A box of pulser LEDs is used to inject light at one end of a fiber and the response is measured at both ends. The light is also injected into a PIN Diode that measures the LED light level. (b) The response of the PMT shown against the PIN diode response.

The rest of the fanned out fibers are connected to the light injection manifolds at the end of each scintillator module, such that their light shines directly on to the WLS fiber. The LEDs are pulsed at multiple light levels, and the response of the diode is measured against the response of the PMT in order to calibrate the PMT gain (Figure 
5.1.b). The light injection calibration system pulses the full detector over several light levels once every month. The monthly pulsing data is used to evaluate each PMT gain curve. Additionally, the LEDs are pulsed during normal data taking to evaluate drift in the baseline gain values over time.

\subsubsection{Timing Response Calibration}

To determine if a track is caused by a particle propagating forward $(+\mathrm{Z})$ or backwards $(-Z)$ through the detector, the timing information of each digit has to be well calibrated. The time of each hit is limited by four primary factors: (1) the ADC runs at $640 \mathrm{MHz}$, and so the time is binned by $1.5625 \mathrm{~ns}$ intervals, (2) the fiber excitation and decay time can vary by as much as $8 \mathrm{~ns}$ between different strips, (3) larger signals have a more prompt PMT trigger response than smaller signal (this is known as time walk and can introduce a time uncertainty as much $10 \mathrm{~ns}$ ), (4) various hardware elements in the data acquisition chain may fail and be replaced with newer elements that have different timing characteristics. All of these effects have been accounted for and corrected using high-energy through-going muons. The timing calibration has achieved a resolution of 2.3 ns 93 .

Each PMT channel has gain and timing correction constants for all time spans, stored in the MINOS database. The reconstruction software takes channels hit at a given time from the data output, and matches each channel to the appropriate gain and timing corrections to convert the ADC counts into a measurement of number of photoelectrons and converts the TDC output into a time after the event trigger in nanoseconds.

\section{3 $\quad$ Demultiplexing}

The optical summing of 8 fibers on each of the 16 PMT pixels introduces a strip-digit ambiguity in the events, such that any digit could potentially map to 8 different strips. This must be resolved in order to produce topological information about an event. The 8 strips that are optically summed on one side can use the information from the other side of the strip to help resolve ambiguity. The gang of 8 strips that are connected to a single PMT pixel on one side are redistributed on their opposite strip end according 
(a) East Side : PMT 0 Strip Map

\begin{tabular}{|c|c|c|c|}
\hline Pixel 0 & Pixel 1 & Pixel 2 & Pixel 3 \\
\hline $\begin{array}{c}13970 \\
162 \quad 03 \\
185 \quad 116 \quad 47\end{array}$ & $\begin{array}{l}58{ }^{127}{ }^{35} \\
{ }^{81}{ }^{32} 12 \\
104 \quad 173 \quad 150\end{array}$ & $\begin{array}{ccc}186 & 163 & 140 \\
117 & 71 \\
1 & 24 & 94\end{array}$ & $\begin{array}{l}36 \quad 82 \quad 128 \\
105 \quad 59 \\
174 \quad 13 \quad 151\end{array}$ \\
\hline Plane 2 & Plane 2 & Plane 2 & Plane 2 \\
\hline Pixel 4 & Pixel 5 & Pixel 6 & Pixel 7 \\
\hline $\begin{array}{ccc}175 & 152 & 129 \\
14 & 60 \\
83^{4} & 37 & 106\end{array}$ & $\begin{array}{lll}95 & 2 & 48 \\
164 & 141 \\
25 & 118 & 187\end{array}$ & $\begin{array}{c}15 \quad 107 \quad 38 \\
61 \quad 130 \\
176 \quad 84 \quad 153\end{array}$ & 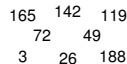 \\
\hline Plane 2 & Plane 2 & Plane 2 & Plane 2 \\
\hline Pixel 8 & Pixel 9 & Pixel 10 & Pixel 11 \\
\hline \begin{tabular}{lll}
73 & 4 & 50 \\
\multicolumn{2}{c}{143} & 27 \\
96 & 166 & 189 \\
\end{tabular} & $\begin{array}{lll}131 & 154 & 108 \\
85 & 62 \\
39^{2} & 16 & 177 \\
\end{array}$ & $\begin{array}{ccc}5 & 28 & 120 \\
& 74 & 167 \\
51 & 97 & 190 \\
\end{array}$ & $\begin{array}{ccc}178 & 40 & 17 \\
132 & 109 \\
155 & 63 & 86 \\
\end{array}$ \\
\hline Plane 2 & Plane 2 & Plane 2 & Plane 2 \\
\hline Pixel 12 & Pixel 13 & Pixel 14 & Pixel 15 \\
\hline $\begin{array}{ccc}87 & 110 & 64 \\
156 & 133 \\
179 & 41 \quad 18\end{array}$ & $\begin{array}{ccc}98 & 144 & 191 \\
6 & 52 & \\
121 & 29 & 75\end{array}$ & $\begin{array}{lll}157 & 65 & 42 \\
& 111 & 134 \\
180 & 88 & 19\end{array}$ & $\begin{array}{ccc} & 30 & 145 \\
53 & 99 \\
168 & 76 \quad 122\end{array}$ \\
\hline Plane 2 & Plane 2 & Plane 2 & Plane 2 \\
\hline
\end{tabular}

(c) East Side : PMT 1 Strip Map

\begin{tabular}{|c|c|c|c|}
\hline Pixel 0 & Pixel 1 & Pixel 2 & Pixel 3 \\
\hline $\begin{array}{lll}169 & 100 & 77 \\
54 & 8\end{array}$ & $20 \quad 181 \quad 112$ & $\begin{array}{lll}32 & 124 & 9 \\
& 170 & 101\end{array}$ & $\begin{array}{lll}67 & 44 & 159 \\
136 & 21\end{array}$ \\
\hline $\begin{array}{ccc}31 \quad 146 \quad 123 \\
\text { Plane } 2\end{array}$ & $\begin{array}{lll}43 & 158 & 135 \\
\end{array}$ & \begin{tabular}{lll}
55 & $78 \quad 147$ \\
\multicolumn{3}{c}{ Plane 2}
\end{tabular} & $\frac{113 \quad 182 \quad 90}{\text { Plane } 2}$ \\
\hline & & Plane 2 & Plane 2 \\
\hline Pixel 4 & Pixel 5 & Pixel 6 & Pixel 7 \\
\hline $\begin{array}{ccc}114 \quad 22 & 45 \\
68 & 91 \\
160 \quad & 93 & 137\end{array}$ & 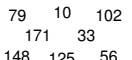 & $\begin{array}{ccc}138 \quad 23 & 46 \\
184 & 115\end{array}$ & $\begin{array}{lll}103 \quad 172 \quad 126 \\
& 11 & 149\end{array}$ \\
\hline $\begin{array}{ccc}160 \quad 183 \quad 137 \\
\text { Plane } 2\end{array}$ & $\begin{array}{c}148 \quad 125 \quad 56 \\
\text { Plane } 2\end{array}$ & $\begin{array}{c}161 \quad 92 \quad 69 \\
\text { Plane 2 }\end{array}$ & $\begin{array}{ccc}57 \quad 34 \quad 80 \\
\text { Plane 2 }\end{array}$ \\
\hline Pixel 8 & Pixel 9 & Pixel 10 & Pixel 11 \\
\hline $\begin{array}{c}185 \quad 162 \quad 70 \\
116 \quad 47 \\
139 \quad 93 \quad 0\end{array}$ & $\begin{array}{lll}35 & 12 & 150 \\
81 & 173 & \\
58 & 127 & 104\end{array}$ & $\begin{array}{c}117 \quad 140 \\
{ }_{1}^{186} \quad \mathbf{2 4} \\
1^{463} \quad{ }^{94}\end{array}$ & $\begin{array}{c}13 \quad 36 \quad 151 \\
174 \quad 128 \\
105 \quad 59 \quad 82\end{array}$ \\
\hline Plane 4 & Plane 4 & Plane 4 & Plane 4 \\
\hline Pixel 12 & Pixel 13 & Pixel 14 & Pixel 15 \\
\hline $\begin{array}{ccc}175 & 60 & 14 \\
152 & 106 \\
129 & 37 \quad 83\end{array}$ & 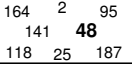 & $\begin{array}{ccc}61 & 84 & 107 \\
15 & 153 \\
38 & 130 \quad 176\end{array}$ & $\begin{array}{ccc}188 & 165 & 142 \\
26 & 72 \\
3 & 49 & 119\end{array}$ \\
\hline $\begin{array}{l}\text { Plane } 4 \\
\end{array}$ & Plane 4 & Plane 4 & ane 4 \\
\hline
\end{tabular}

(e) East Side : PMT 2 Strip Map

\begin{tabular}{|c|c|c|c|}
\hline Pixel 0 & Pixel 1 & Pixel 2 & Pixel 3 \\
\hline $\begin{array}{ccc}4 & 166 & 73 \\
27 & 50 \\
143^{2} & 96 \quad 189\end{array}$ & $\begin{array}{ccc}131 & 154 & 177 \\
62 & 39 \\
16 & 108 & 85 \\
16\end{array}$ & $\begin{array}{ccc}120 & 5 & 97 \\
190 & 28 & \\
74 & 167 & 51\end{array}$ & $\begin{array}{ccc}155 & 40 & 63 \\
109 & 86 \\
17 \quad 178 \quad 132\end{array}$ \\
\hline Plane 4 & Plane 4 & Plane 4 & Plane 4 \\
\hline Pixel 4 & Pixel 5 & Pixel 6 & Pixel 7 \\
\hline $\begin{array}{ccc}179 & 110 & 64 \\
87 & 133 \\
41 & 156 \quad 18 \\
\end{array}$ & $\begin{array}{llll}29 & 144 & 6 \\
52 & 98 \\
191 & 75 & 121 \\
\end{array}$ & $\begin{array}{ccc}134 & 65 & 42 \\
180 & 19 & \\
88 & 111 & 157 \\
\end{array}$ & $\begin{array}{ccc}168 & 30 & 122 \\
& 145 \quad 53 \\
7 & 99 & 76 \\
\end{array}$ \\
\hline Plane 4 & Plane 4 & Plane 4 & Plane 4 \\
\hline Pixel 8 & Pixel 9 & Pixel 10 & $\begin{array}{l}\text { Pixel } 11 \\
\end{array}$ \\
\hline $\begin{array}{ccc}100 & 54 & 31 \\
& 8 & 123 \\
77 & 169 & 146\end{array}$ & $\begin{array}{ccc}181 & 135 & 66 \\
89 & 112 \\
43 & 158 & 20\end{array}$ & $\begin{array}{lll}9 & 55 \quad 78 \\
101 & 147 \\
32 & 124 & 170\end{array}$ & $\begin{array}{ccc}182 & 67 & 44 \\
90 & 136 \\
21 & 113 & 159\end{array}$ \\
\hline Plane 4 & Plane 4 & Plane 4 & Plane 4 \\
\hline Pixel 12 & Pixel 13 & Pixel 14 & Pixel 15 \\
\hline $\begin{array}{ccc}45 & 160 & 114 \\
183 & 137 \\
22 & 68 & 91\end{array}$ & $\begin{array}{ccc}56 \quad 148 & 33 \\
79 & 125 \\
171 & 102 & 10\end{array}$ & $\begin{array}{ccc}1611^{23} & 184 \\
115 & 69 \\
138 \quad 92 & 46\end{array}$ & $\begin{array}{l}80 \quad 11 \quad 34 \\
103 \quad 57 \quad{ }^{34} \quad 126 \quad 172\end{array}$ \\
\hline Plane 4 & Plane 4 & Plane 4 & Plane 4 \\
\hline
\end{tabular}

(b) West Side : PMT 0 Strip Map

\begin{tabular}{|c|c|c|c|}
\hline Pixel 0 & Pixel 1 & Pixel 2 & Pixel 3 \\
\hline $\begin{array}{lll}120 & 96 & 168 \\
& 24 & 144\end{array}$ & $\begin{array}{lll}180 & 12 & 84 \\
132 & 36\end{array}$ & ${ }^{1}{ }^{73} \quad 25 \quad 169$ & $\begin{array}{ccc}13 \quad 37 \quad 157 \\
181 \quad 109\end{array}$ \\
\hline $\begin{array}{lcl}0 & 72 \quad 48 \\
& \text { Plane 2 }\end{array}$ & $\begin{array}{ccc}108 \quad 156 \quad 60 \\
\text { Plane } 2\end{array}$ & $\begin{array}{c}12149 \quad 97 \\
\text { Plane } 2\end{array}$ & $\begin{array}{ccc}61 \quad 133 \quad 85 \\
\text { Plane } 2\end{array}$ \\
\hline Pixel 4 & Pixel 5 & Pixel 6 & Pixel 7 \\
\hline $\begin{array}{ccc}14 & 38 & 134 \\
& 62 & 86 \\
110 & 182 & 158 \\
\end{array}$ & $\begin{array}{ccc}2 & 50 & 122 \\
98 & 146 \\
74 & 170 \quad 26\end{array}$ & $\begin{array}{lll}111 & 135 & 87 \\
15 & 183 \\
39 & 159 & 63\end{array}$ & $\begin{array}{llll}51 & 99 & 27 \\
123 & 3 & \\
75 & 147 & 171 \\
\end{array}$ \\
\hline Plane 2 & Plane 2 & Plane 2 & Plane 2 \\
\hline Pixel 8 & Pixel 9 & Pixel 10 & Pixel 11 \\
\hline \begin{tabular}{lll}
52 & 4 & 100 \\
\multicolumn{1}{c}{148} & 124 \\
76 & 172 & 28
\end{tabular} & $\begin{array}{lll}136 & 40 & 184 \\
16 & 160 \\
112 & 64 & 88\end{array}$ & $\begin{array}{l}101{ }^{53}{ }^{29} \\
{ }^{77} \quad{ }^{125} \quad{ }^{125}\end{array}$ & 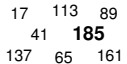 \\
\hline Plane 2 & Plane 2 & Plane 2 & Plane 2 \\
\hline Pixel 12 & Pixel 13 & Pixel 14 & Pixel 15 \\
\hline $\begin{array}{ccc}42 & 18 & 114 \\
138 & 66 \\
186 & 162 & 90\end{array}$ & $\begin{array}{lll}150 & 102 & 78 \\
& 6 & 174 \\
54 & 126 & 30\end{array}$ & 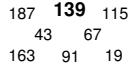 & 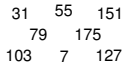 \\
\hline$P$ & & & 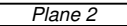 \\
\hline
\end{tabular}

(d) West Side : PMT 1 Strip Map

\begin{tabular}{|c|c|c|c|}
\hline Pixel 0 & Pixel 1 & Pixel 2 & Pixel 3 \\
\hline $\begin{array}{ccc}80 & 152 & 56 \\
176 & 8 & \\
104 & 32 & 128\end{array}$ & $\begin{array}{ccc}164 & 44 & 140 \\
68 & 92 \\
116 & 20 & 188\end{array}$ & $\begin{array}{ccc}9 & 153 & 33 \\
& 177 & 129 \\
105 & 81 & 57\end{array}$ & $\begin{array}{ccc}117 & 93 & 69 \\
& 141 & 165 \\
21 & 189 & 45\end{array}$ \\
\hline Plane 2 & Plane 2 & Plane 2 & Plane 2 \\
\hline Pixel 4 & Pixel 5 & Pixel 6 & Pixel 7 \\
\hline $\begin{array}{lll}142 & 118 & 166 \\
46 & 190 \\
22 & 70 & 94\end{array}$ & $\begin{array}{ccc}154 & 178 & 130 \\
34 & 58 \\
106 & & 82\end{array}$ & $\begin{array}{lll}167 & 119 & 47 \\
143 & 23 & \\
191 & 71 & 95\end{array}$ & $\begin{array}{ccc}131 & 35 & 11 \\
179 & 155 \\
59 & 107 & 83\end{array}$ \\
\hline Plane 2 & Plane 2 & Plane 2 & Plane 2 \\
\hline Pixel 8 & Pixel 9 & Pixel 10 & Pixel 11 \\
\hline $\begin{array}{l}96 \quad 48 \quad 120 \\
144 \quad 168 \\
24 \quad 72 \quad 0\end{array}$ & $\begin{array}{ccc}12 & 132 & 108 \\
36 & 156 \\
180 & & 84\end{array}$ & 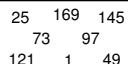 & 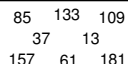 \\
\hline Plane 4 & Plane 4 & Plane 4 & Plane 4 \\
\hline Pixel 12 & Pixel 13 & Pixel 14 & Pixel 15 \\
\hline $\begin{array}{ccc}38 & 110 & 86 \\
14 & 134 \\
182 & 62 & 158\end{array}$ & $\begin{array}{ccc}26 & 2 & 122 \\
170 & 74 \\
146 & 98 & 50\end{array}$ & $\begin{array}{lll}15 & 39 & 63 \\
& 111 & 135 \\
87 & 159 & 183\end{array}$ & 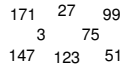 \\
\hline Plane 4 & Plane 4 & Plane 4 & Plane 4 \\
\hline
\end{tabular}

(f) West Side : PMT 2 Strip Map

\begin{tabular}{|c|c|c|c|}
\hline Pixel 0 & Pixel 1 & Pixel 2 & Pixel 3 \\
\hline $\begin{array}{ccc}100 & 4 & 76 \\
148 & 124\end{array}$ & $\begin{array}{cc}160 \quad 184 & 16 \\
88 \quad 136\end{array}$ & $\begin{array}{ccc}125 & 53 & 77 \\
5 & 149\end{array}$ & $\begin{array}{ccc}113 & 161 & 65 \\
185 & 137\end{array}$ \\
\hline $\begin{array}{lll}28 & 52 & 172 \\
\end{array}$ & $\begin{array}{lll}40 & 64 & 112 \\
\end{array}$ & $101 \quad 173$ & $\begin{array}{ll}89 & 17 \\
\end{array}$ \\
\hline Plane 4 & Plane 4 & Plane 4 & Plane 4 \\
\hline Pixel 4 & Pixel 5 & Pixel 6 & Pixel 7 \\
\hline $\begin{array}{ccc}162 & 186 & 66 \\
114 & 42\end{array}$ & $\begin{array}{lll}54 & 174 & 6 \\
150 & 102\end{array}$ & ${ }_{19}^{19}{ }_{163}^{67} 43^{115}$ & $\begin{array}{ccc}103 & 127 & 31 \\
& & \\
\end{array}$ \\
\hline $\begin{array}{lll}18 & 90 & 138 \\
\end{array}$ & $\begin{array}{lll}126 & 78 & 30 \\
\end{array}$ & $\begin{array}{lll}187 & 139 & 91 \\
\end{array}$ & $\begin{array}{lll}175 & 79 & 151\end{array}$ \\
\hline Plane 4 & Plane 4 & Plane 4 & Plane 4 \\
\hline Pixel 8 & Pixel 9 & Pixel 10 & Pixel 11 \\
\hline $\begin{array}{ccc}32 & 128 & 104 \\
176 & 152\end{array}$ & $\begin{array}{cc}116 \quad 164 & 140 \\
20 & 68\end{array}$ & $\begin{array}{ccc}105 & 81 & 57 \\
129 & 177\end{array}$ & $\begin{array}{ccc}165 & 117 & 189 \\
21 & 93\end{array}$ \\
\hline $80 \quad 56$ & $\begin{array}{lll}44 & 188 & 92 \\
\end{array}$ & $33 \quad 153$ & $\begin{array}{lll}69 & 141 & 45 \\
\end{array}$ \\
\hline Plane 4 & Plane 4 & Plane 4 & Plane 4 \\
\hline Pixel 12 & Pixel 13 & Pixel 14 & Pixel 15 \\
\hline $\begin{array}{ccc}118 & 166 & 70 \\
190 & 94 & \\
142 & 22 & 46\end{array}$ & $\begin{array}{ccc}82 & 58 & 130 \\
106 & 178 \\
10 & 154 & 34\end{array}$ & 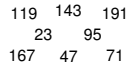 & 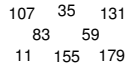 \\
\hline Plane 4 & Plane 4 & Plane 4 & Plane 4 \\
\hline
\end{tabular}

Figure 5.2: Optical summing pattern on pixel for PMT MUX boxes on opposing strip ends. Two planes are split over the three PMTs in a MUX box, with the 192 strips in a plane matched in 8 strips groups to 24 pixels. 
to the following rules: (1) none of the 8 will share a PMT on the opposite, (2) the 8 pixels that the 8 fibers are attached to will not be adjacent pixels in the M16 PMT, and (3) the 8 strips must be separated in the plane by at least 23 strips on both ends. In Figure 5.2 this pattern in demonstrated with the two MUX boxes that are attached to the opposite ends of strips.

Adjacent pixels in a single PMT experience a small amount of cross-talk that can confuse the demultiplexing process. This cross-talk can be caused by light leaking from one pixel to a neighboring pixel, or the electrical signal on the PMT anode leaking into nearby anodes. In either case, pixels with a signal caused solely by cross-talk are identified by small pulse-height signals accompanied by a large pulse-height signal on one of the adjacent pixels. Pixels that are horizontally or vertically adjacent are known have a $1 \%$ cross-talk leakage, while diagonally adjacent pixels are know to have $0.1 \%$ cross-talk leakage 93. Before demultiplexing, cross-talk hits are identified, and the cross-talk pulse-height is added to the pixel that was deemed to have caused the cross-talk.

The AltDeMux software package [94] was created specifically for the atmospheric neutrino analysis. The purpose of the demultiplexer software is to match each hit pixel with an appropriate strip (a hit-strip solution). The software starts by tagging hits that can be easily resolved with concise hit information on both strips ends. A strip that is read out on both ends is called "double-ended". If pixel A on the east side of the detector is hit, there will be 8 pixels on the west side of the detector that could have been hit. If only one of these pixels is hit within $4 \mathrm{~ns}$ of the time that pixel A was hit, then this forms an unambiguous hit-strip solution. Double-ended hits with an un-ambiguous strip solution are tagged "golden hits".

The golden hits define target regions in the detector, and the remaining hits are examined against these regions. A plane with unresolved hits should have golden hits in the surrounding planes of the same view. These surrounding hits establish an acceptable region for hits to occur, and more strip-hit solutions are found in the remaining ambiguous hits. The collection of hits that have been demultiplexed are used to identify a provisional event type, and this event type guides the demultiplexer in finding a strip-hit solution for the remaining single-ended strips.

The demultiplexer performance is measured by identifying the digits that have been 

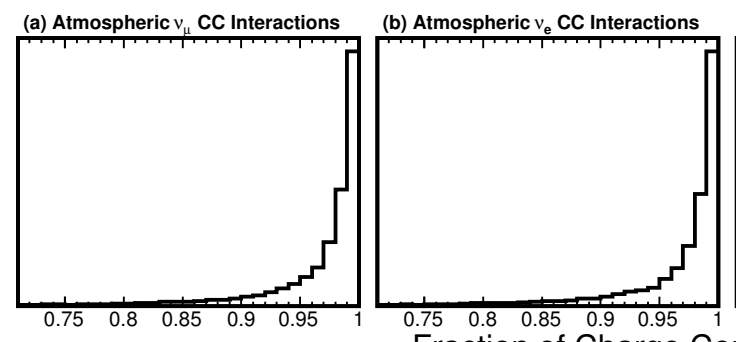

(c) Atmospheric $v$ NC Interactions

(d) Cosmic-ray $\mu$ Interactions Fraction of Charge Correctly Demultiplexed
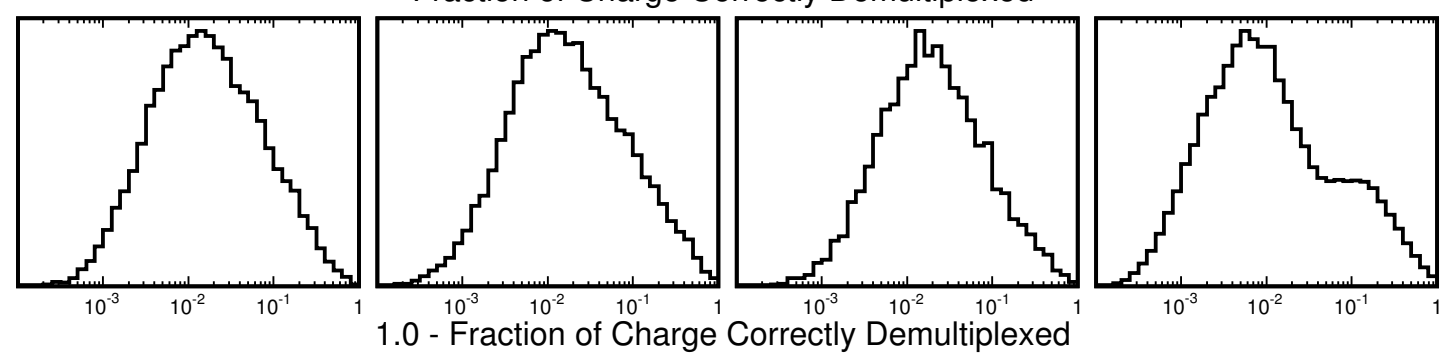

Figure 5.3: Performance of the demultiplexer software measured by the fraction of charge that is correctly demultiplexed.
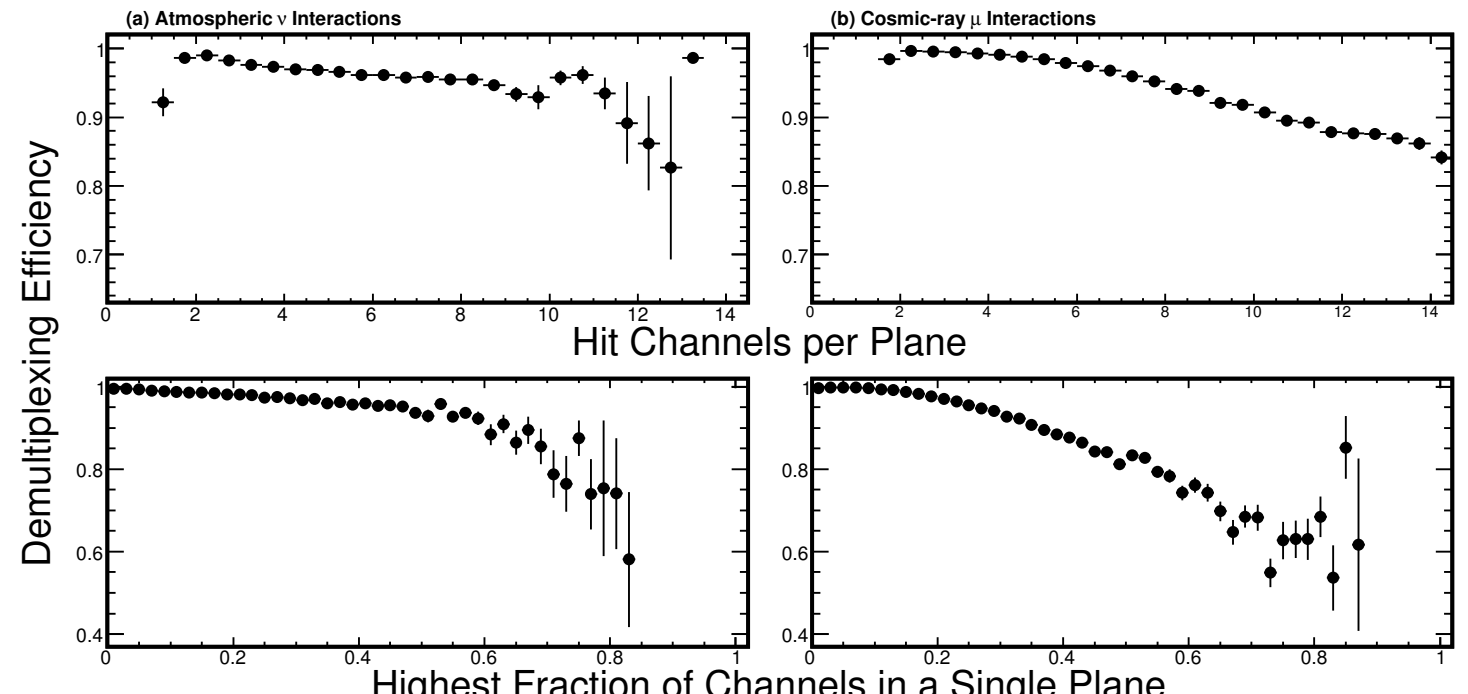

Figure 5.4: Demultiplexer performance versus the mean digits per plane and maximum digit fraction in a single plane for (a) atmospheric neutrino and (b) cosmic-ray muon interactions. 
correctly matched to the strip that was hit, with the true information in the simulated events. The "demultiplexing efficiency" is defined as the fraction of charge that has been correctly demultiplexed to the total charge in the event. This efficiency is shown in Figures $5.3 \mathrm{a}, \mathrm{b}, \mathrm{c}$, and $\mathrm{d}$ for atmospheric $\nu_{\mu} \mathrm{CC}, \nu_{e} \mathrm{CC}, \nu \mathrm{NC}$, and cosmic-ray $\mu$ interactions respectively. The mean efficiency of demultiplexing is slightly higher for both cosmic-ray muons and $\nu_{\mu} \mathrm{CC}$ interactions (98\%) than for the showering $\nu_{e} \mathrm{CC}$ and $\nu$ NC interaction (97\%).

The demultiplexing procedure relies on the unambiguous "golden hits" to correctly resolve the remaining ambiguous hits, and events that have few of these golden hits tend towards a lower demultiplexing efficiency. The number of unambiguous strip-digit solutions in a plane falls with the number of digits that are hit in that plane, and accordingly a plane with several digits is expected to produce fewer golden hits than a plane with just a few digits. This effect is demonstrated with atmospheric neutrino and cosmic-ray muon interactions in Figures [5.4] a and 5.4,b. The demultiplexing efficiency for both interactions types falls as the mean digits per plane increases. The efficiencies are also lower for events that have a large fraction of the digits hit in a single plane.

\subsection{Construction of Tracks and Showers}

Triggered events can be broken down into one of two types: track-like events or showering events. A track-like event has a straight and narrow signature from each active detector element to the next. A showering event has a diffuse pattern and is spread across many active-detector elements that do not fall onto a straight line. The purpose of separating events into these two classes is to distinguish between the types of neutrino interactions: a $\nu_{\mu} \mathrm{CC}$ interaction will primarily produce a track-like event, and a $\nu_{e} \mathrm{CC}$ or $\nu \mathrm{NC}$ interaction will produce a primarily showering event.

A constructed track is the collection of the hits in the detector that are best described as a track-like event, and a constructed shower is the collection of hits that are best described as a showering event. The reconstruction software creates track and shower objects by first joining small 2D clusters in each view. Based on the topology of these $2 \mathrm{D}$ clusters, they are each labeled either a $2 \mathrm{D}$ track or a $2 \mathrm{D}$ shower. The $2 \mathrm{D}$ tracks and showers from the U-view that match in time and space with $2 \mathrm{D}$ tracks and showers in 
the $\mathrm{V}$-view are joined together to form 3D tracks and showers.
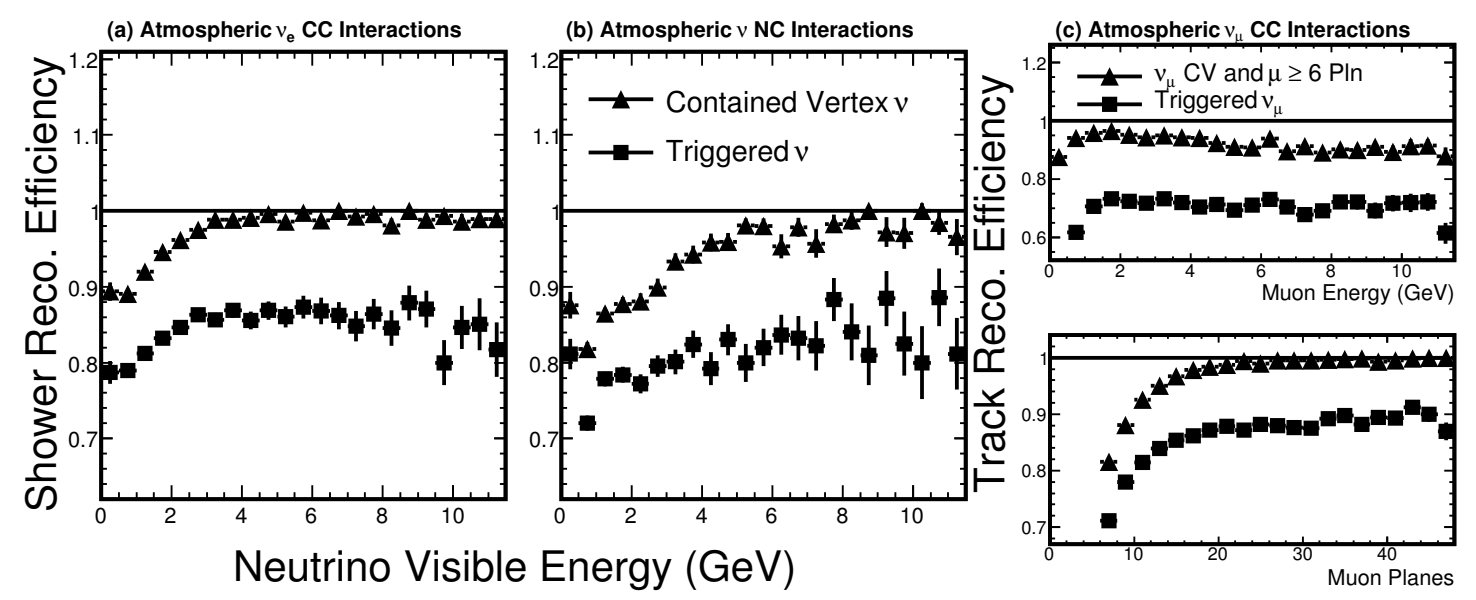

Figure 5.5: Reconstruction efficiency for track and shower as a function of visible neutrino energy $(\mathrm{a}, \mathrm{b})$ and muon energy $(\mathrm{c})$. The figures include reconstruction efficiencies for the triggered neutrino set and also for triggered neutrinos that interact in the detector fiducial volume.

Tracks and showers are intended to be monolithic objects that identify the components of neutrino interactions. A $\nu_{\mu}$ CC interaction results in a muon component that should reconstruct as a track and a hadronic component that should reconstruct as a shower. The $\nu_{e} \mathrm{CC}$ and $\nu \mathrm{NC}$ interactions result only in components that should reconstruct as showers. Reconstruction performance has been evaluated for both tracks and shower by the efficiency of reconstructing a track or shower in the appropriate neutrino interaction.

The efficiencies for reconstructing showers in atmospheric $\nu_{e} \mathrm{CC}$ and $\nu \mathrm{NC}$ interactions are shown in Figures [5.5] a and $\mathrm{b}$ as a function of the visible neutrino energy. The shower reconstruction efficiencies are shown for two different samples: (1) the set of atmospheric $\nu_{e} \mathrm{CC}$ or $\nu \mathrm{NC}$ interactions that produced a trigger and survive the noise filter and (2) the subset of (1) where the neutrino interacted at least 5 planes from a supermodule longitudinal edge ( $\mathrm{Z}$ direction), and $50 \mathrm{~cm}$ from a supermodule traverse edge (X, Y, U, or V direction). The second subset is referred to as the contained neutrino vertex set. Atmospheric $\nu_{e} \mathrm{CC}$ interactions with a contained vertex reconstruct a shower with near $100 \%$ efficiency for a visible neutrino energy above $2 \mathrm{GeV}$. However, the contained vertex $\nu \mathrm{NC}$ interaction shower reconstruction efficiency do not approach 
$100 \%$ until $E_{\nu \text { visible }} \gtrsim 5 \mathrm{GeV}$.

The track reconstruction efficiency for $\nu_{\mu} \mathrm{CC}$ interactions is shown in Figure 5.5]c as a function of the muon energy. The two track efficiencies in Figure [5.5]c are for the efficiency of reconstructing a track in two samples: (1) the set of atmospheric $\nu_{\mu} \mathrm{CC}$ interactions that produced a detector trigger and survive the noise filter and (2) the subset of (1) with a contained neutrino interaction vertex and a muon that traverses at least 6 planes. The efficiency for reconstructing a track doesn't reach $100 \%$ for $\nu_{\mu} \mathrm{CC}$ as the energy increases. Also shown in Figure 5.5. c is the track reconstruction efficiency as a function of the true number of planes that the muon traverses in the detector. The track reconstruction efficiency approaches $100 \%$ for events where the muon traverses 20 planes.

The second measurement of the track and shower reconstruction performance considers how well the tracks and showers match the true event. The set of strips that compose a track or a shower are compared to the actual strip hits. A track is compared to the strips that were hit by a muon from a $\nu_{\mu} \mathrm{CC}$ interaction, and a shower is compared to the strips that were hit by the showering particles from a $\nu_{e} \mathrm{CC}$ interaction. The sum of the charge of all hits associated with a reconstructed event is labeled $Q_{\text {RecoShw }}$ and $Q_{\text {RecoTrk }}$ for showers and tracks respectively. The sum of the charge associated with a true interaction is labeled $Q_{\text {TrueShw }}$ for a $\nu_{e}$ CC interaction and $Q_{\text {TrueTrk }}$ for the muon hits from a $\nu_{\mu} \mathrm{CC}$ interaction. The sum of the charge from the hits in a reconstructed shower or track that are also in the true shower or track is $Q_{\text {RecoTrueShw }}$ and $Q_{\text {RecoTrueTrk }}$ respectively.

If a strip is incorrectly demultiplexed, but is still reconstructed into the appropriate track or shower event, then that hit is not used in the sum $Q_{\text {RecoTrue }}$. An incorrectly demultiplexed hit will almost never be associated with the correct track, due to the minimum 23 strip physical separation of the 8-fold strip ambiguity. However, showering neutrino interactions will occasionally have an incorrectly demultiplexed strip still included in the shower. This happens most often with neutral current interactions, because a hadronic shower is more spread out than an electromagnetic shower from a $\nu_{e}$ CC interaction.

The shower and track performance is quantified by the "purity" $\left.\left(Q_{\text {RecoTrue }}\right) / Q_{\text {Reco }}\right)$ and the "completeness" $\left(Q_{\text {RecoTrue }} / Q_{\text {True }}\right)$. The purity measures how much of the 

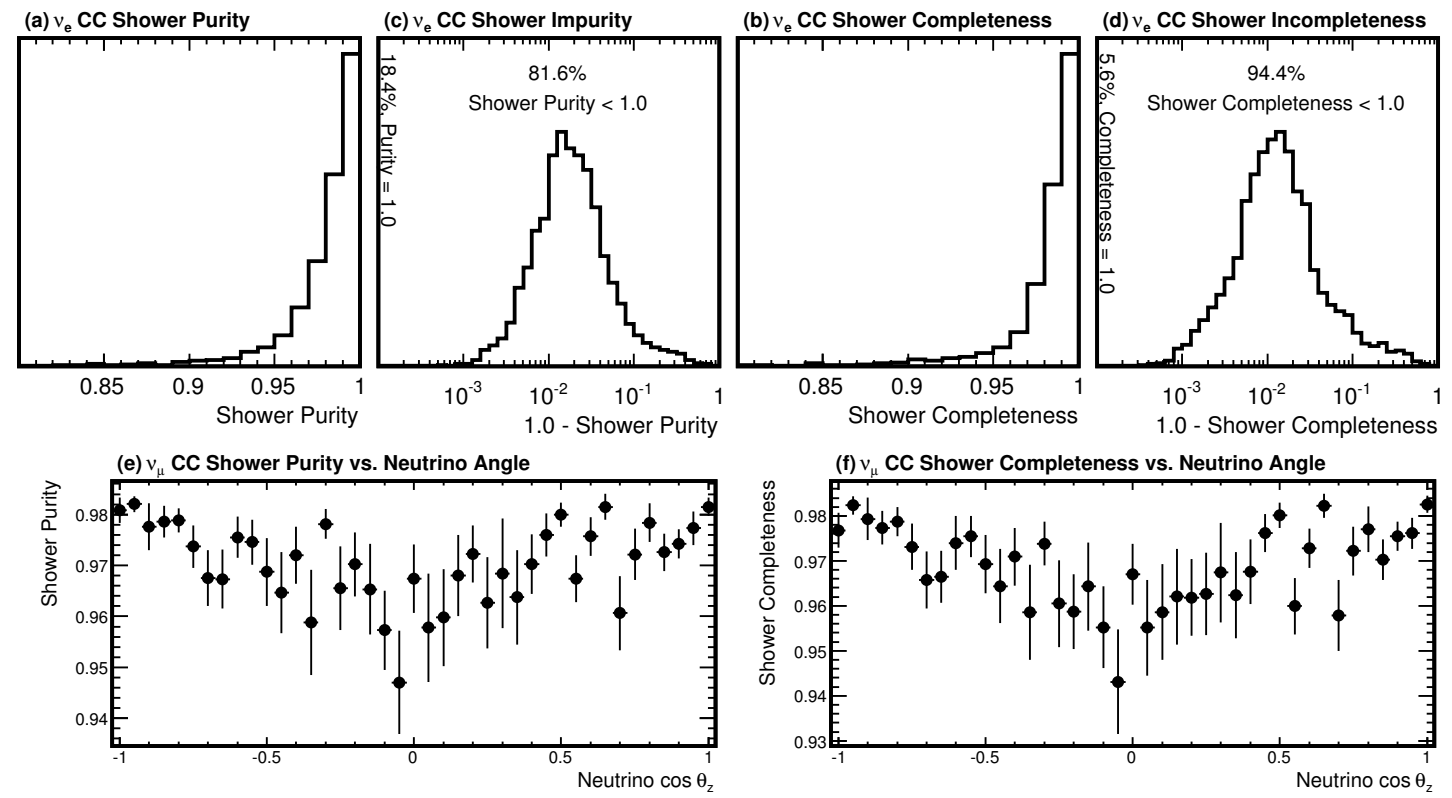

Figure 5.6: Performance of shower finding in terms of completeness and purity relative to simulated atmospheric $\nu_{e}$ CC interactions that reconstruct a single shower.

(a) Reco - True Shower Vertex Depth

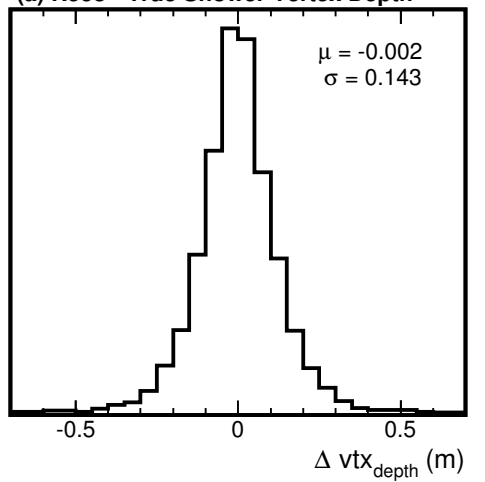

(b) Reco - True Shower Vertex Z

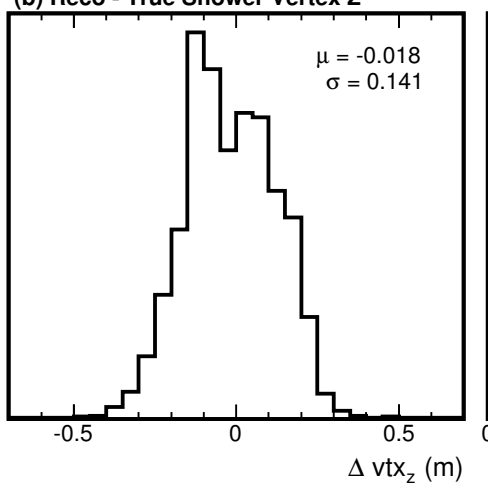

(c) Vertex $\Delta$ Reco $\leftrightarrow$ True

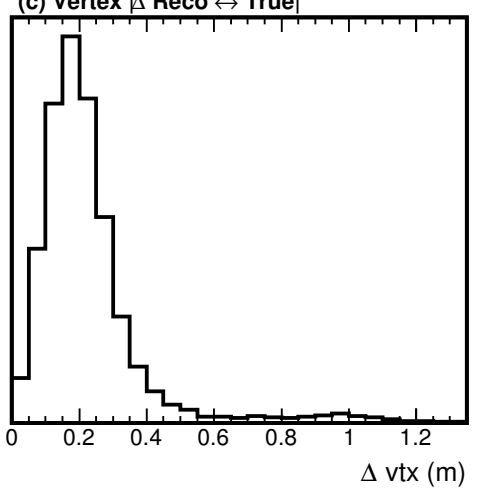

Figure 5.7: Performance for finding the vertex of a simulated atmospheric $\nu_{e} \mathrm{CC}$ interactions that reconstruct a single shower. 

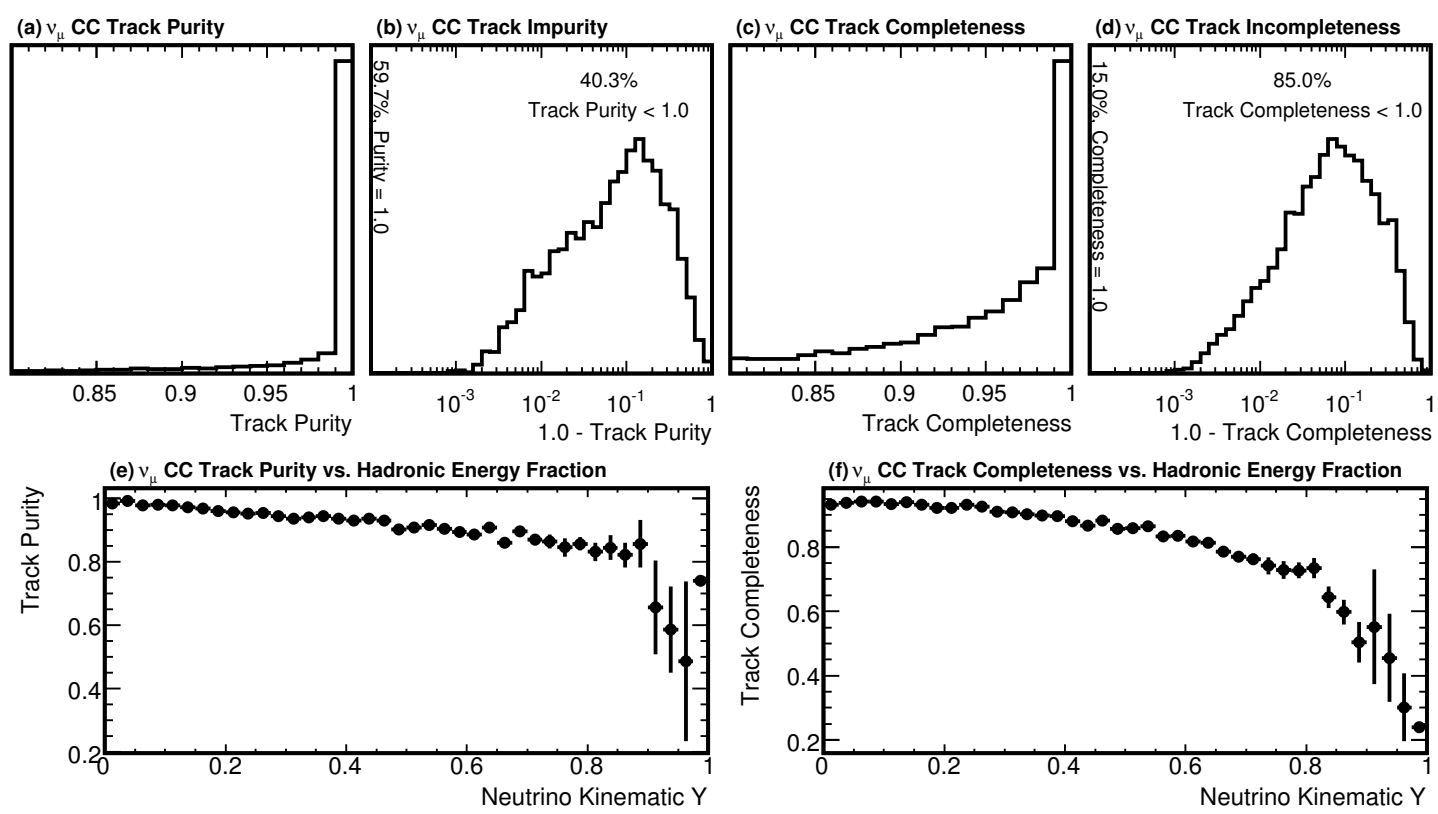

Figure 5.8: Performance of track finding in terms of completeness and purity relative to simulated atmospheric $\nu_{\mu} \mathrm{CC}$ interactions that reconstruct a single track.

(a) Reco - True Track Vertex Depth

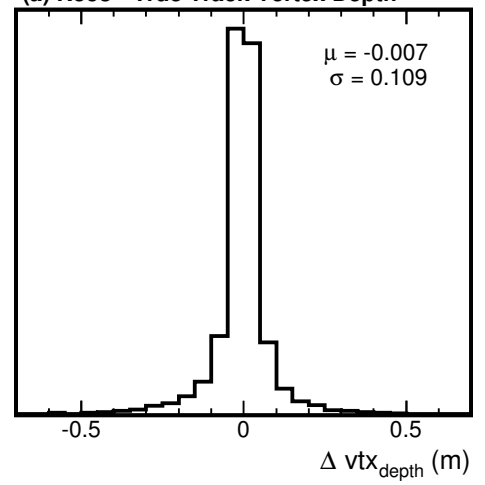

(b) Reco - True Track Vertex Z

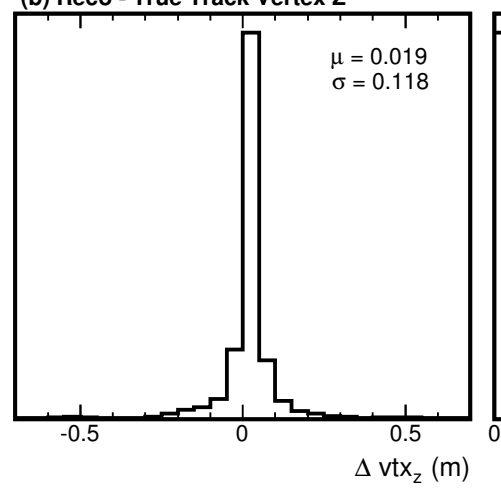

(c) Vertex $\triangle$ Reco $\leftrightarrow$ True

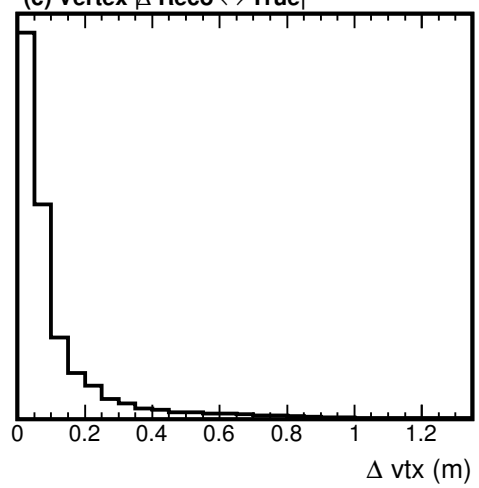

Figure 5.9: Performance for finding the vertex of a simulated atmospheric $\nu_{\mu}$ CC interactions that reconstruct a single track. 
charge in the reconstructed events is caused by the true event, while the completeness measures how much of the true event in contained in the reconstructed event. Due to the restriction on wrongly demultiplexed strips, the completeness and purity are convoluted with the demultiplexing efficiency. The completeness and purity are measured for tracks and showers using the same set of simulated atmospheric neutrino events that was to evaluate the performance of the demultiplexer. Track events used for this evaluation must cross at least 8 planes, and shower events must cross at least 5 planes.

Figure [5.6] a shows the distribution of shower purity for $\nu_{e} \mathrm{CC}$ interactions. This distribution is strongly favored towards a value of 1.0, and many $\nu_{e}$ interactions have perfectly pure reconstructed showers. To demonstrate more detailed structure in the purity distribution, Figure [5.6. b shows the distribution of (1.0 - shower purity) on a logarithmic scale. On this scale, perfectly pure events (purity $=1.0$ ) are off the left end of the X-axis range in the histogram. The shower completeness and (1.0 - completeness) are shown in Figures [5.6] and 5.6. d. The $\nu_{e} \mathrm{CC}$ events that reconstruct a shower with low purity or completeness tend to be from neutrinos with a steep angle to the plane face, as demonstrated in Figures [5.6] e and [5.6. f.

Tracks reconstructed from $\nu_{\mu} \mathrm{CC}$ interactions tend to have a higher fraction of events that are perfectly pure than showers reconstructed from $\nu_{e} \mathrm{CC}$ interactions, as shown in Figures 5.8, a and 5.8. b. For deep-inelastic scattering $\nu_{\mu}$ CC interactions, a fraction of the neutrino energy produces a hadronic shower at the interaction vertex. Figure 5.8. e demonstrates that as the fraction of the energy given to the hadronic shower increases, the track purity decreases. The track completeness is similarly affected by the hadronic shower component (Figure $5.8 \mathrm{f}$ ).

The final performance measure of the track and shower reconstruction considers the ability to locate the true neutrino interaction vertex. The vertex location performance is defined by three quantities: (1)the difference between the true and reconstructed vertex depths, (2)the distance between the true and reconstructed vertices in the $\mathrm{Z}$ coordinate, and (3)the linear distance between the true and reconstructed vertices. Figure [5.7 shows these quantities for showers reconstructed from $\nu_{e}$ CC interactions, and Figure 5.9 for tracks reconstructed from $\nu_{\mu} \mathrm{CC}$ interactions. The track vertex location outperforms the shower vertex location for all three quantities.

For reconstructed showers, the distribution of the Z-vertex displacement is bifurcated 
about $0.0 \mathrm{~m}$. A showering neutrino interaction occurs at a single interaction point, and progresses through the detector in the same direction of the interacting neutrino. The reconstructed vertex point doesn't attempt to find this initial interaction point, because the shower is often to short to determine which side the process may have started from. The shower vertex is instead determined by the charge weighted mean $3 \mathrm{D}$ position of the shower hits. The actual neutrino interaction point will be either in front of or behind the reconstructed vertex location in $\mathrm{Z}$, which leads to the bifurcated distribution in Figure $5.7 \mathrm{~b}$.

The performance of the vertex location is important for making reliable fiducial selections. The poor performance of the Z-coordinate location of the shower vertex can be dealt with by requiring that the full extent of the shower be a certain distance from the supermodule plane faces (discussed in Section [7.2). The vertex performance also affects how the cosmic-ray veto shield tags events as vetoed (discussed in Section [7.6). Locating the track vertex is a more involved process than locating the shower vertex, as the timing information is used to identify one side of the track as the "track vertex" and the other side as the "track end".

\subsection{Direction and Energy Reconstruction}

The final step in the reconstruction of tracks and showers is to determine for each of these an energy and direction that most closely resembles that of the incident particle that induced the track or shower. A track is caused by a muon, which loses an expected amount of energy each time it passes through a steel plane. For fully-contained (FC) tracks, this can be exploited to measure the track momentum directly by quantifying the amount of material traversed by the track, but for partially-contained (PC) tracks this is not possible. The momentum for PC tracks is instead measured by the curvature of the muon track in the magnetic field. The energy determination for shower objects is simpler, the true shower energy is fit to a polynomial function of the sum of the shower charge.

The calibration of the shower energy was calculated individually for showers from $\nu_{e} \mathrm{CC}$ interactions, and $\nu \mathrm{NC}$ interactions. In both cases, a large sample of simulated atmospheric neutrinos was used to perform this calibration. Reconstructed showers had 
to cross at least 5 planes. The result was two separate calibrated energy values $\left(E_{E M}\right.$ from $\nu_{e} \mathrm{CC}$ and $E_{\text {had }}$ from $\nu \mathrm{NC}$ interactions). The resulting energy reconstruction performance for $E_{E M}$ is demonstrated in Figure [5.10 for $\nu_{e} \mathrm{CC}$ interactions.

The energy resolution for a sampling calorimeter is usually expressed as $\frac{\sigma_{E}}{E}=\frac{C}{\sqrt{E}}$, because the resolution is limited by the statistical sampling rate of the calorimeter. If the energy resolution is also dependent on the measurement of noisy quantities, then the energy resolution will include a term that scales with the energy $\left(\frac{\sigma_{E}}{E}=C\right.$. These two resolution terms are typically added in quadrature, since the effects that lead to the two resolution terms are independent. The result is the calorimetric resolution function $\frac{\sigma_{E}}{E}=A \oplus \frac{B}{\sqrt{E}}$, where the $\oplus$ denotes addition in quadrature. The energy resolution in Figure [5.10 c is fitted as function of the true neutrino energy, and the calorimetric resolution function [19] is shown in Equation [5.2. Figure 5.11] shows the $E_{\text {had }}$ reconstruction performance for the $\nu$ NC interactions. The calorimetric resolution for the visible neutrino energy in neutral-current interaction is shown in equation 5.1 .

$$
\begin{aligned}
& \frac{\sigma_{E}}{E}=20 \% \oplus \frac{45 \%}{\sqrt{E}} \quad\left(E_{E M} \text { for } \nu_{e} C C\right) \\
& \frac{\sigma_{E}}{E}=21 \% \oplus \frac{55 \%}{\sqrt{E}} \quad\left(E_{H a d} \text { for } \nu N C\right)
\end{aligned}
$$

The minimum ionizing energy loss in pure iron is $11.4 \mathrm{MeV} / \mathrm{cm}$ [19], and so for a muon traveling straight through the detector, the initial muon momentum could simply be reconstructed $p_{\mu}=N_{\text {planes }} * 2.54 \mathrm{~cm} /$ planes $* 11.4 \mathrm{MeV} / \mathrm{cm}$. The reality is not this simple for a few reasons: the muon doesn't travel straight through the steel planes, the detector contains materials other that steel (i.e. polystyrene and aluminum), and the energy loss in MINOS steel isn't exactly $11.4 \mathrm{MeV} / \mathrm{cm}$ because not all muons are minimum ionizing.

A more reliable method to measure the momentum of a fully-contained (FC) track from the range through the detector is by using a statistical calibration from a set of Monte Carlo simulated events, similar to the shower energy calibration procedure. Using the charge weighted mean 3D position of each active plane in a track, the full path length that a muon travelled through steel plane can be determined. The sum of the path lengths over all of the track planes is called the "track range". The FC track 


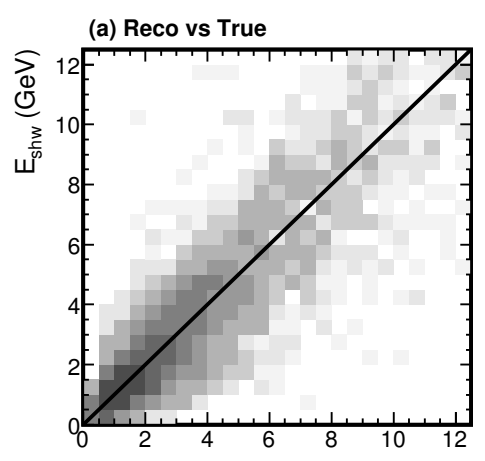

(b) [Reco - True] / True vs True

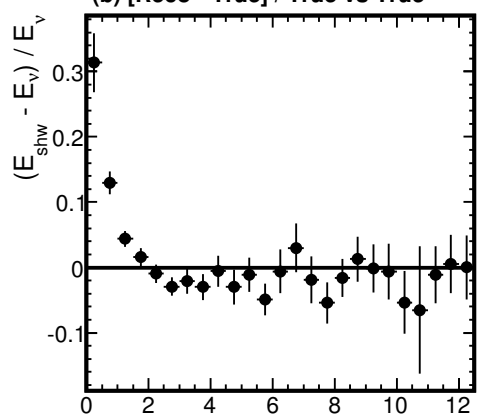

(c) Resolution vs. True

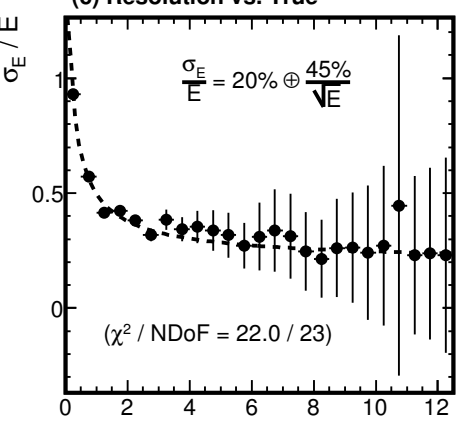

True Visible Neutrino Energy

Figure 5.10: Performance for reconstructing the neutrino energy in a $\nu_{e} \mathrm{CC}$ interaction.
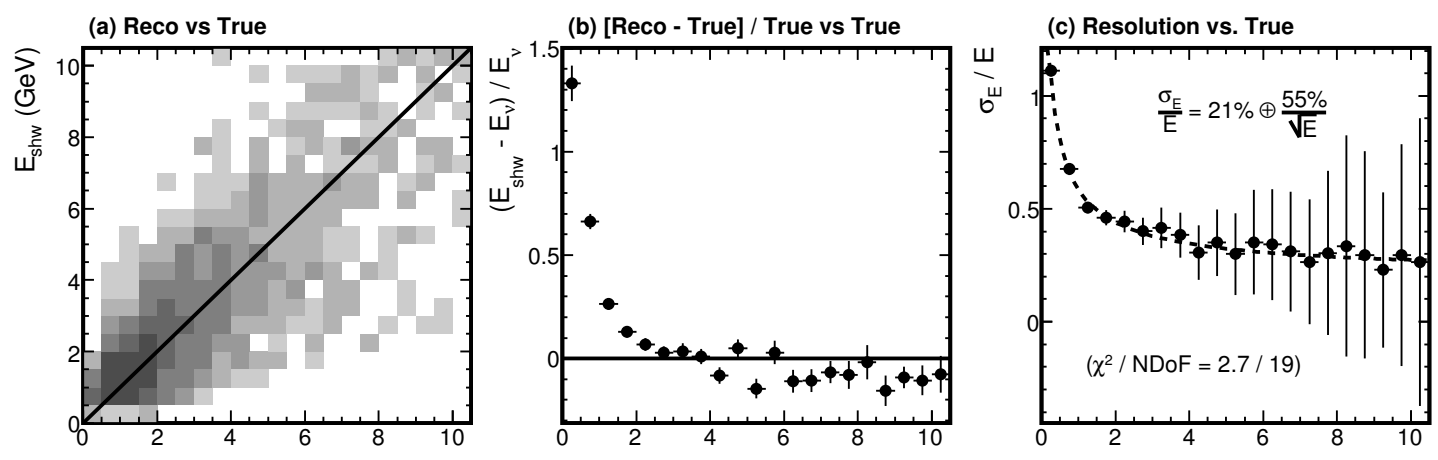

\section{True Visible Neutrino Energy}

Figure 5.11: Performance for reconstructing the visible neutrino energy in a $\nu$ NC interaction.

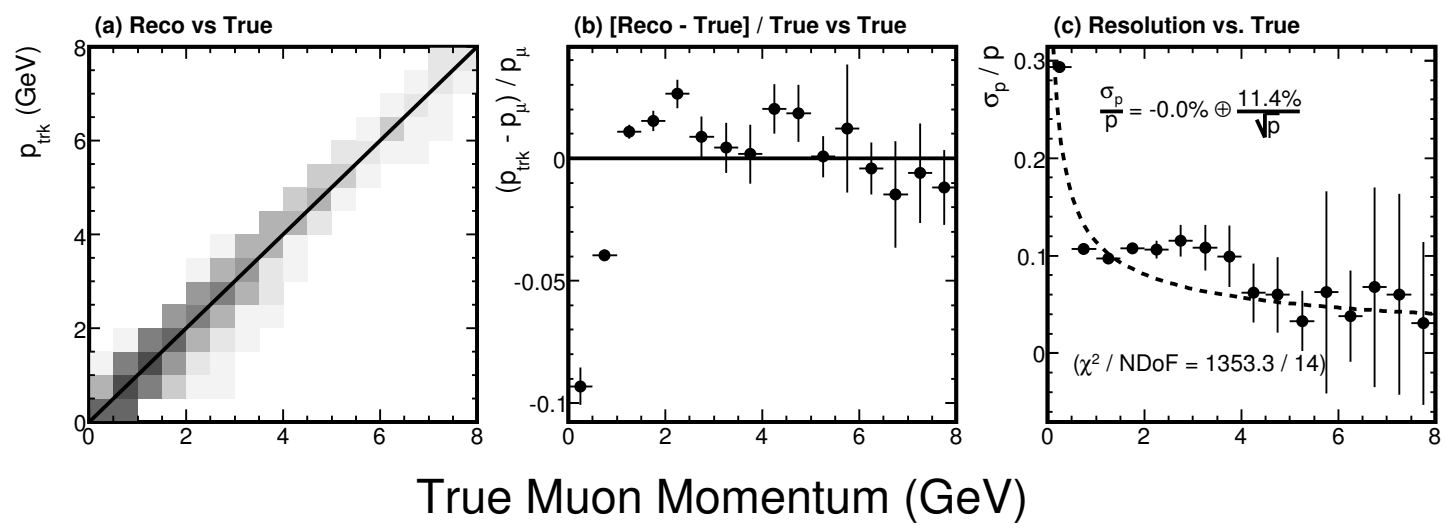

Figure 5.12: Performance for reconstructing the muon momentum from a fully-contained muon resulting from a $\nu_{\mu} \mathrm{CC}$ interaction. 


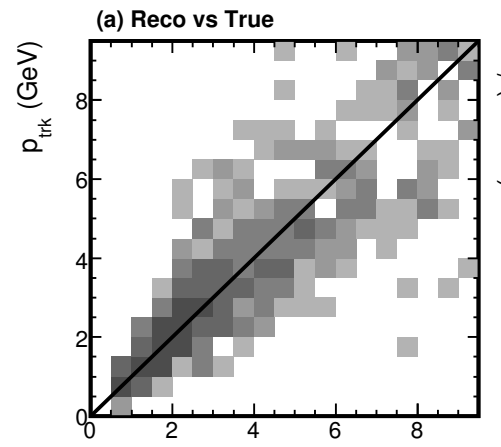

(b) [Reco - True] / True vs True

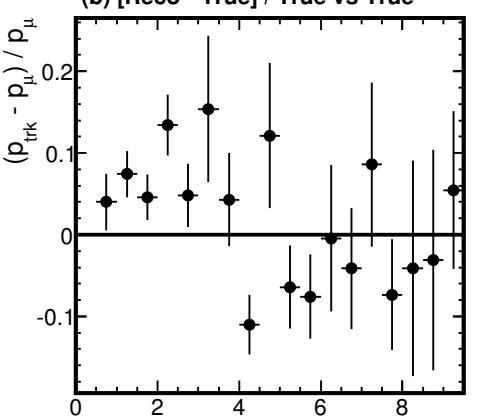

(c) Resolution vs. True

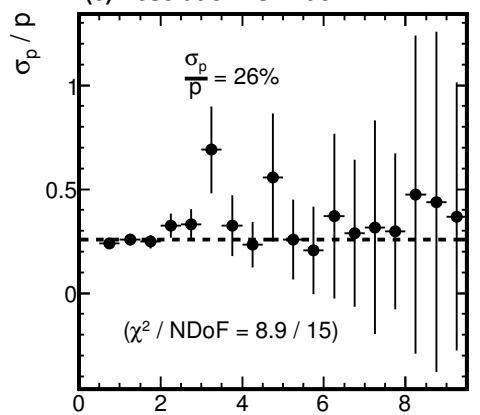

True Muon Momentum (GeV)

Figure 5.13: Performance for reconstructing the muon momentum from a partiallycontained muon resulting from a $\nu_{\mu} \mathrm{CC}$ interaction.

momentum $\left(p_{t r k F C}\right)$ found by fitting the true muon energy to a polynomial function of the track range. The performance of the FC muon momentum reconstruction is shown in Figure 5.12 A fit was made of the calorimetric resolution function to the data in Figure $5.12 \mathrm{c}$, with the result shown on that figure and in Equation 5.3. There is a feature in the resolution spectrum that significantly reduces the goodness of this fit. For muons with an energy range $2 \mathrm{GeV}<E_{\mu}<4 \mathrm{GeV}$, the resolution is much worse than the fit value. This feature is not understood, and warrants further investigation.

$$
\begin{array}{ll}
\frac{\sigma_{p}}{p}=0.0 \% \oplus \frac{11.4 \%}{\sqrt{p}} \quad\left(E_{F C} \text { for } \nu_{\mu} C C\right) \\
\frac{\sigma_{p}}{p}=26 . \% \quad\left(E_{P C} \text { for } \nu_{\mu} C C\right)
\end{array}
$$

The momentum for tracks that are partially contained is obtained by measuring the curvature of the track in the magnetic field. A charged particle traveling through a magnetic field $\vec{B}(s)$ with momentum $\vec{p}(s)$ (where $s$ is the distance traversed along the particle's trajectory) will change its momentum according to Equation 5.5. The first term is from the relativistic bending of the muon in a magnetic field, and the second term is from the continuous muon energy loss to the steel. The Cambridge reconstruction divides the track into segments, and finds value of $\frac{Q}{P}$ and $\sigma_{\frac{Q}{P}}$ for each segment. The mean value of the $\frac{Q}{P}$ is combined with a mean energy loss term $\left(\mu_{T}\right)$ to find $\frac{Q}{P_{0}}$. 


$$
\begin{aligned}
\frac{d \vec{p}(s)}{d s} & =0.3 Q \hat{p}(s) \times \vec{B}(s)+\hat{p}(s) T(p) \\
\frac{Q}{p(s)} & =\frac{\frac{d \hat{p}(s)}{d s} \cdot \hat{p}(s) \times \vec{B}(s)}{0.3|\hat{p}(s) \times \vec{B}(s)|} \\
\frac{Q}{p_{0}} & =\frac{\left\langle\frac{Q}{p}\right\rangle}{1+\left|\left\langle\cdot \mu_{T} \cdot s \frac{Q}{p}\right\rangle\right|}
\end{aligned}
$$

The momentum reconstruction performance for partially-contained tracks is shown in figure 5.13. The resolution fit as function of the momentum from Figure 5.13. is expressed in Equation 5.4. The statistical error term $\left(\frac{1}{\sqrt{p}}\right)$ vanishes for this fit, which is expected since this method does not rely on sampling.

The reconstruction of the direction for tracks and showers is performed with a linear least squares fit in each view of the points around the vertex. The two $2 \mathrm{D}$ directions are combined to form a single 3D direction. The 3D direction finding performance is evaluated by evaluating the dot product of the true and reconstructed direction unit vectors to give $\cos (\Delta \theta)$, a The performance of the shower direction for $\nu_{e} \mathrm{CC}$ and $\nu \mathrm{NC}$ interactions are shown in Figures [5.14] a and 5.14]d respectively. For both interaction types $\sim \frac{1}{3}$ of the showers are reconstructed at least $90^{\circ}$ away from the correct direction. Figures [5.14] b and 5.14] show that showers that are steeper to the plane face have a lower direction reconstruction efficiency. The direction reconstruction efficiency is also lower for lower energy interactions (Figures 5.14. $\mathrm{c}$ and 5.14. $\mathrm{g}$ ).

The small percent of tracks the have $\vec{d}_{t r k} \cdot \vec{d}_{\mu}<0$ in Figure 5.9 a are from tracks with the wrong side selected as vertex. The track direction reconstruction efficiency depends strongly on the number of planes that the muon has crossed in the detector (Figure [5.9. b), and also the initial energy of the muon (Figure [5.9] $\mathrm{c}$ ). 

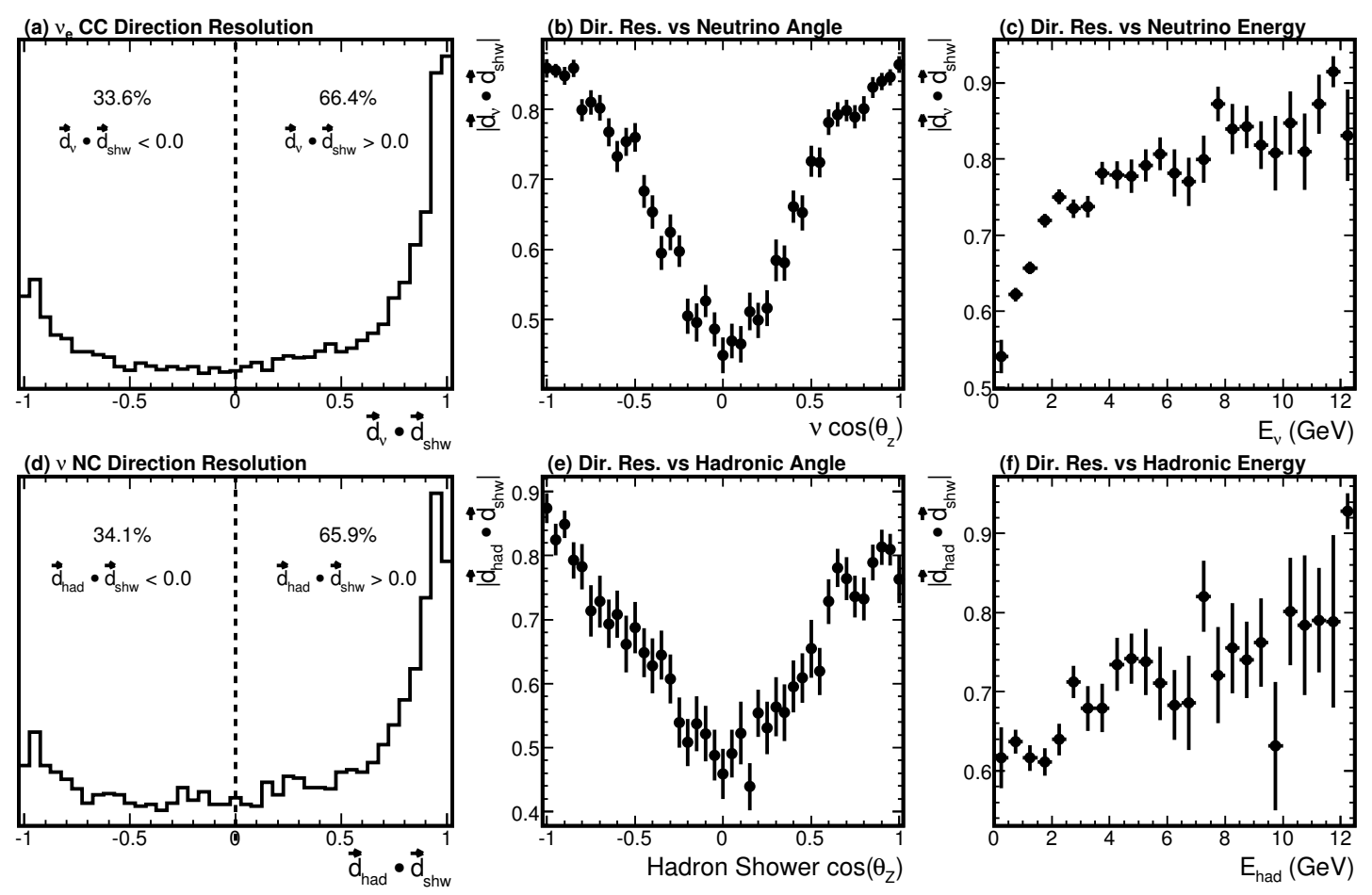

Figure 5.14: Performance for reconstructing the neutrino direction from $\nu_{e} \mathrm{CC}$ and $\nu$ $\mathrm{NC}$ interactions.
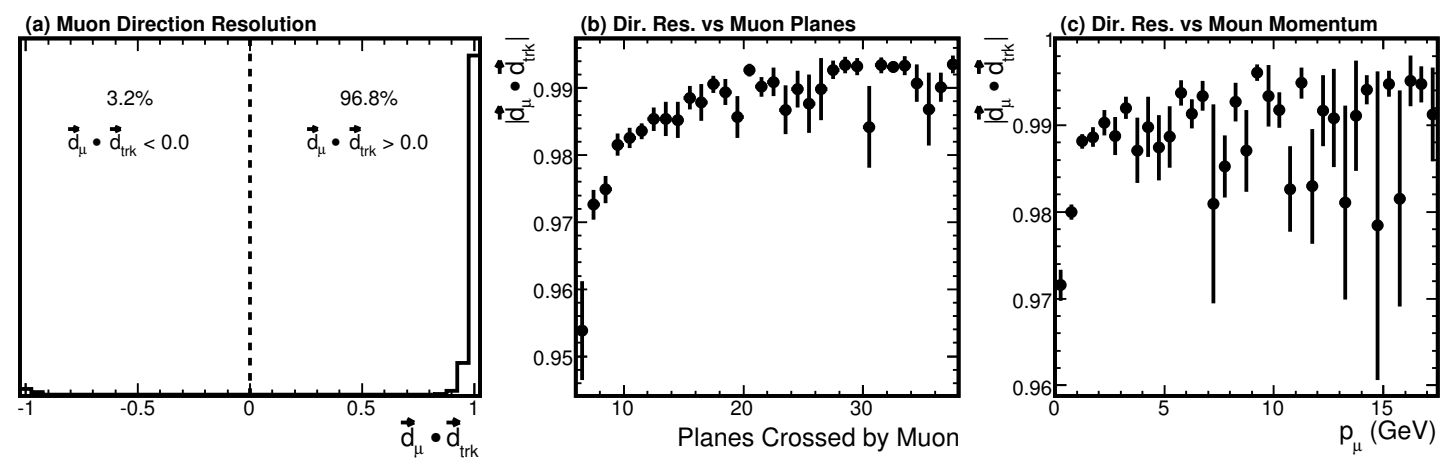

Figure 5.15: Performance for reconstructing the muon direction from a muon resulting from a $\nu_{\mu} \mathrm{CC}$ interaction. 


\section{Chapter 6}

\section{Data Analysis Strategy}

\subsection{Analysis Strategy}

This thesis is based on the observation of atmospheric electron neutrino interactions. Previous experimental observations of neutrino oscillation have found that for the energies and distance scales of atmospheric neutrinos detected in underground observatories, a fraction of muon neutrinos are likely to oscillate into another neutrino flavor while electron neutrinos are not likely to oscillate. The observation of atmospheric electron neutrinos can thus offer little information about neutrino oscillation, but can offer much information about the nature of atmospheric neutrinos without the spectral distortion produced by neutrino oscillation. With this in mind, there are three primary analysis goals of this thesis:

1. Find the atmospheric neutrino flavor double ratio, which is the observer over expected ratio for the ratios of the muon neutrino interaction rate over the electron neutrino interaction rate. $\left(\frac{R_{\nu_{\mu} / \nu_{e}}^{\text {data }}}{R_{\nu_{\mu} / \nu_{e}}^{M C}}\right)$

2. Study atmospheric neutrino oscillation based on the observed interaction rates of electron and muon neutrinos.

3. Measure the atmospheric neutrino flux based on the electron neutrino interaction rate. 
A direct measurement of either the muon or electron-neutrino interaction rate would require the selection of a set of pure neutrino interaction events. In practice this is not possible. The event selection is thus divided into track-like and showering events, where the track-like events are primarily $\nu_{\mu} \mathrm{CC}$ interactions and the showering events are primarily $\nu_{e} \mathrm{CC}$ events. To accomplish the three analytic goals above, the following procedures have been implemented.

1. Establish clean sets of observed and simulated signal and background events (Chapter [6).

2. Select contained vertex showering and track-like events from the cleaned sets (Sections $\mathbf{7 . 2}$ for vertex containment, 7.4 for showering events selection, and 7.5 for track-like event selection).

3. Use triggered hits in the veto shield to remove cosmic rays from the selected events and estimate the cosmic-ray contribution to the remaining events (Section 17.6).

4. Measure the atmospheric neutrino double ratio and make systematic variances to estimate the double-ratio systematic error (Section 17.7.1).

5. Use the observed double-ratio to measure the Neyman frequentist confidence intervals in oscillation parameter space, and measure the atmospheric neutrino flux based on optimal oscillation scenarios (Section [8.2).

6. Perform a maximum likelihood fit of the neutrino oscillation parameters and neutrino flux to the neutrino interaction rates in order to simultaneously measure the atmospheric neutrino flux and neutrino oscillation parameters (Section 8.3).

\section{- Event Selection Strategy -}

To minimize the bias in the event selection process, simulated data must be used to optimize the selection values. The simulated data include atmospheric neutrinos that were used to evaluate selection efficiency, to quantify the reconstruction performance, and to compare to the observed events. The simulated sets also include cosmic-ray muon that are used to evaluate selection efficiency and reconstruction performance, but are not used in the end to compare to the resulting selected set. In order for the data 
and simulated sets to be considered "clean", they must satisfy two criteria: (1) the data event rate must be consistent throughout the data run, and (2) spectral differences between the simulated and observed must be minimized.

The MINOS far detector was designed and optimized for the muon-neutrino disappearance analysis of beam neutrino events, and is not optimal for the selection of showering neutrino interactions. The radiation length in iron is $X_{0}=1.76 \mathrm{~cm}$, which is $\sim 70 \%$ of the steel plane thickness. The radiation length is defined as the length over which the electron energy is reduced by a factor of 1 /e due to bremsstrahlung radiation. An electron will lose energy dominantly via bremsstrahlung radiation in iron until it reaches the "critical energy" $\left(E_{C} \sim 30 \mathrm{MeV}\right)$, at which point the ionization loss to the material starts to dominate and the electron quickly loses its remaining energy.

As the electron loses energy to bremsstrahlung radiation, multiple photons are also produced by this process. These photons lose their energy dominantly through three methods: $e^{+} / e-$ pair production $\left(E_{\gamma}>150 \mathrm{MeV}\right)$, incoherent scattering $(150 \mathrm{keV}$ $\left.<E_{\gamma}<150 \mathrm{MeV}\right)$, or photoelectric absorption $\left(E_{\gamma}<150 \mathrm{keV}\right)$. The mean free path for pair production is $\frac{9}{7} * X_{0}$. The high-energy photons from bremsstrahlung should pair produce, and the newly created electron will travel through the detector the same as the initial electron. An iterative process that creates multiple charged particles results from the initial electron interaction. These separate charged particles cannot be individually identified due to the coarseness of the active detector elements, and so the cumulative particle stream is instead called a shower.

The showering event selection process relies on the characteristics of an electromagnetic cascade to distinguish $\nu_{e} \mathrm{CC}$ interaction from showering events caused by cosmic-ray muons. The steel plane thickness is greater than the radiation length for iron, and an low energy electromagnetic cascade may not develop fully enough to be able to distinguish. Because of this, the showering event selection process is additionally challenging for low energy showering events.

The process for selecting track-like events is more straight forward than the showering event selection process, as the detector was designed to be a muon calorimeter. Track-like event selection is divided into three groups defined by track containment and event direction. Fully-contained (FC) and partially-contained downward-going (PCDN) tracks share a selection process, as the background to both of these event types is from 
steep cosmic-ray muons. Atmospheric neutrinos that are identified as FC and PCDN have been isolated from the cosmic-ray background with topology variables pertaining to the hits and charge surrounding the event vertex. Partially-contained upward-going (PCUP) tracks are selected separately. The primary background to PCUP tracks is from downward cosmic-ray muons that mis-reconstruct with the wrong direction; thus the atmospheric neutrino events are identified in this sample by timing quality variables. The track selection process is based on the analysis developed for $\nu_{\mu}$ charged-current atmospheric neutrinos 95.

Selected events in both track-like and showering samples shared two conflicts after the event selection: (1) there was still a large contribution of cosmic-ray events, and (2) the size of this contribution was not well known. Use of the cosmic-ray veto shield solved both of these issues. Each of the four shield sections were used to identify a cosmic-ray induced event by a shield hits that coincide spatially and temporally with the track or shower vertex. With a well measured efficiency for tagging cosmic-ray muons and an accidental tagging rate of contained vertex neutrinos, the contribution of the cosmic-ray to remaining events was obtained. The shield selection process was not applied to the PCUP track events,

\section{- Atmospheric Neutrino Flux Measurement Strategy -}

The remaining track-like and showering events were used to measure the atmospheric neutrino double ratio. To obtain the expected number of track-like and showering event rates, the simulated neutrino samples were used in combination with the cosmic-ray background measured with the shield selection process. Due to the non-trivial manner in which the expected event rates are obtained, the statistical error and confidence for the rejection of null oscillation were found with Monte Carlo techniques.

The atmospheric neutrino flux can be measured by the $\nu_{e}$ interaction rate. The selected showering event sample was expected to consist of a non-trivial fraction of $\nu_{\mu}$ interactions (which may experience deficits due to oscillation) as well, and thus could not be directly used to measure the atmospheric neutrino flux. In order to measure the atmospheric neutrino flux, the $\nu_{\mu}$ contribution to the showering sample would have to be weighted by some oscillation probability.

The Monte Carlo techniques that were used to find the statistical error of the double 
ratio also detail a rejection of the null oscillation hypothesis. This technique was next use to measure the confidence for the rejection of other oscillation hypotheses. This expanded technique was the basis for establishing the frequentist probabilities for a range of oscillation parameters. The expected size of the showering sample was determined based on the oscillation probability, and the measurement of the atmospheric neutrino flux was thus obtained.

The first method to measure the atmospheric neutrino flux was done in two stages, a frequentist fit of the double ratio followed by a re-weighting of the expected showering event rate. The verify this result of this method, a second method attempted to resolve the best fit oscillation parameters and atmospheric neutrino flux in a single stage. To do this, the track-like and showering event rates are independently fit to the two oscillation parameters and the atmospheric neutrino flux with the maximum likelihood method. Much like the previous fit, the maximum likelihood fit is under-constrained by one degree of freedom. The best fit flux scale is obtained by projecting the $-2 \Delta \ln (\mathcal{L})$ ellipsoid from the 3D parameter on to the $1 \mathrm{D}$ flux scale space.

\subsection{Data Set used in Analysis}

The data set analyzed to search for atmospheric neutrino events spans from August 2003 to February 2005. This large data sample is predominantly composed of radioactive noise and cosmic-ray muons, with a small contribution from atmospheric neutrinos. The data collection scheme at the far detector gathers event in to run files that are divided by "run" and "subrun". A run can contain any number of subruns, where the subruns are defined by an hour of data collection. A run is defined by the by collection of subruns between a run that measure the PMT pedestals followed by a charge calibration run. The events collected from far detector are required to pass the "physics trigger" described in section 3.2.2 In addition to the trigger requirement, the detector electronics (such as high voltage for the PMT and the current source for the magnetic coil) must all be responsive with the detector control systems.

The Cambridge group has measured the live time of this data set very accurately by counting each 1 second time frame in which the detector is active and taking adequate data by the criteria mentioned above. The full data set from August 2003 to February 
2005 contains 418.5 live days of events. This translates to an exposure of $6.18 \mathrm{kty}$, or a fiducial exposure of $4.51 \mathrm{kty}$ with a fiducial volume of $50 \mathrm{~cm}$ from the lateral supermodule edges, and 5 planes from each supermodule face. The vertex containment selection that establishes the fiducial volume is discussed in Section $\mathbf{7 . 2}$.

\subsection{Monte Carlo Sets used in Analysis}

The analytic basis of this thesis requires that a sample be attained with as many events caused by neutrino interactions as possible and a minimum number of events caused by interactions of other particles. To reduce the full data set to such a sample, selection methods are developed that will achieve the optimal balance of signal (neutrino interactions) and background (other particle interactions). In order to develop these selection methods in a manner which is least biased by data sample, simulated atmospheric neutrinos, cosmic-ray muons, and cosmic-ray-muon induced neutron samples have been generated according to the processes outlined in Chapter [4 All simulation samples shown in this chapter were generated at Cambridge University computing facilities by Pat Ward 96.

\subsubsection{Atmospheric Neutrinos}

\begin{tabular}{|c|c|c|c|c|}
\hline MC Set & Exposure & Flux Model & Had. Int. & Tracking Cut Off \\
\hline A & $1261.5 \mathrm{kty}$ & Bartol $[2]$ & GCALOR $[83]$ & $100 \mathrm{keV}$ \\
$\mathrm{B}$ & $360.5 \mathrm{kty}$ & Bartol & GHEISHA [84] & $100 \mathrm{keV}$ \\
$\mathrm{C}$ & $360 . \mathrm{kty}$ & Bartol & GCALOR & $10 \mathrm{keV}$ \\
$\mathrm{D}$ & $257.6 \mathrm{kty}$ & Battistoni $[38]$ & GCALOR & $100 \mathrm{keV}$ \\
$\mathrm{E}$ & $878 . \mathrm{kty}$ & Bartol $\left(\nu \rightarrow \nu_{\tau}\right)$ & GCALOR & $100 \mathrm{keV}$ \\
$\mathrm{F}$ & $646 . \mathrm{kty}$ & Bartol $\left(\nu_{\mu} \rightarrow \nu_{\tau}\right)$ & GCALOR & $100 \mathrm{keV}$ \\
\hline
\end{tabular}

Table 6.1: Atmospheric neutrino simulation sets

The primary simulated sample used for atmospheric neutrinos has an equivalent exposure of 1264.5 kiloton * year (kty). This set was simulated using a flux model as presented by Barr et al. [2] with a neutrino energy range from $0.2 \mathrm{GeV}$ to $50 \mathrm{GeV}$. Neutrino interactions with nucleons were modeled using Neugen3 [81]. Hadron interactions with atoms were modeled with GCALOR [83. The Geant process that tracks 
particles through the detector followed particles until reaching a $100 \mathrm{keV}$ threshold. In Table 6.1 the primary set is labeled A. Additional sets B through D have systematic variances as described in the table, and are used to investigate systematic errors, which will be discussed at the end of Chapter 7

Sets E and F are systematically identical to Set A, except that neutrinos have been oscillated to tau neutrinos. For set $\mathrm{E}$ all neutrinos have been oscillated, and for set $\mathrm{F}$ only the muon neutrinos have been oscillated. These sets will be used to perform the oscillation analysis in Chapter 8 .

(a) $v_{\mathrm{e}}$ CC Interactions Cut Progression

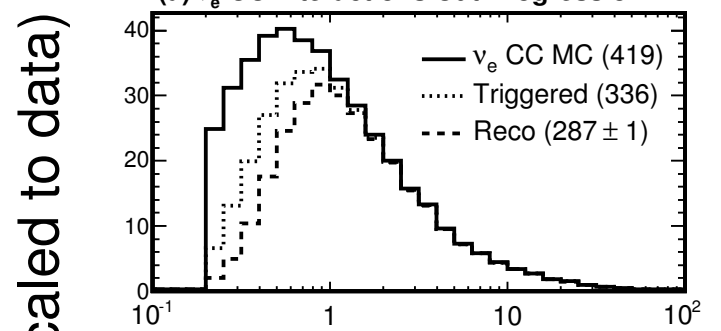

(b) $v_{u}$ CC Interactions Cut Progression

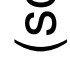

$\div$

ग
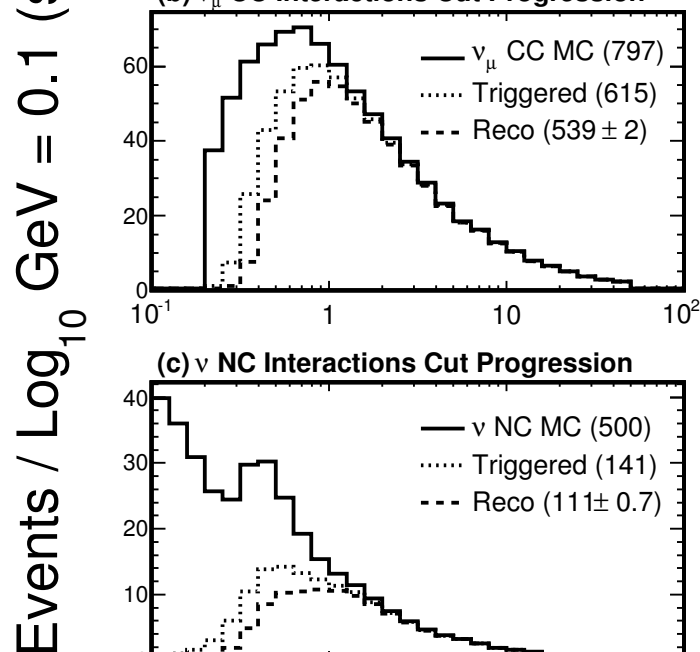

(c) $v$ NC Interactions Cut Progression

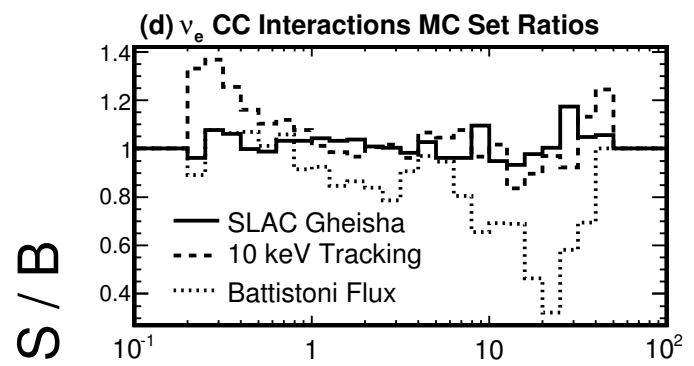

II (e) $v_{u}$ CC Interactions MC Set Ratios
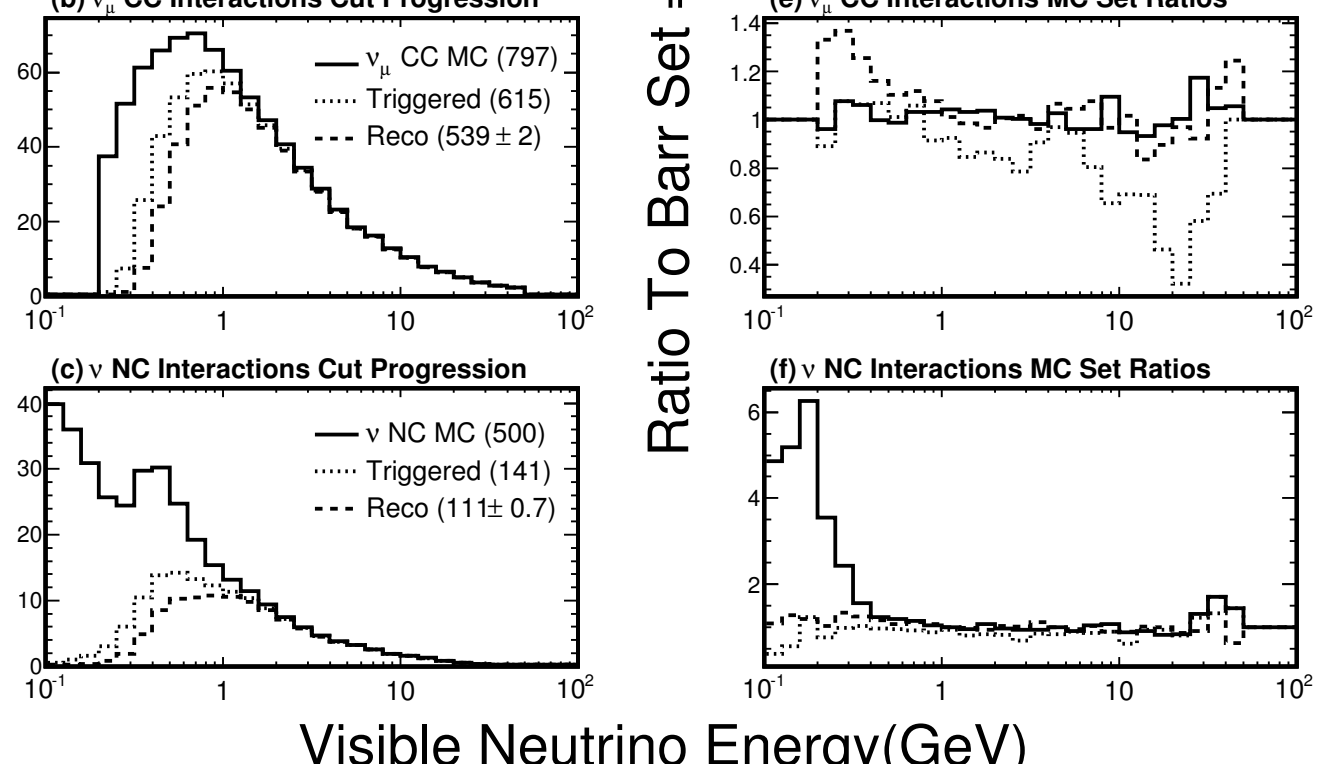

(f) $\vee$ NC Interactions MC Set Ratios

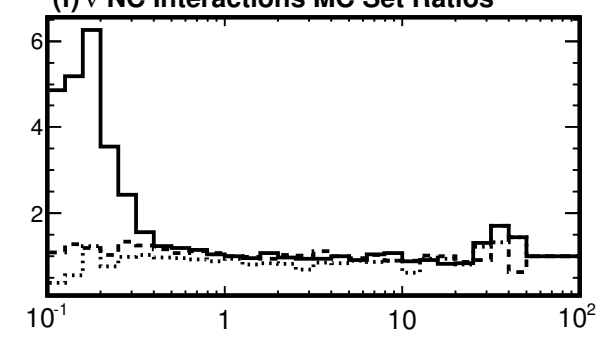

Figure 6.1: Visible neutrino energy spectra across multiple interaction types from generated set to reconstructed set.

The simulated neutrinos interact in the detector and deposit a "Visible Neutrino 
Energy", which is that portion of the neutrino's energy that is visible to the active detector elements. For the MINOS far detector, this amounts to the entire energy of the neutrino for any charged-current neutrino interactions. For neutral-current neutrino interactions, the visible energy is carried by the Z-boson from the neutrino to the nucleon (q in Figure 2.4).

Figures 6.1 a, 6.1 b, and 6.1 c show the true visible neutrino energy spectrum for the electron neutrino charged-current $\left(\nu_{e} \mathrm{CC}\right)$ interactions, muon neutrino charged-current $\left(\nu_{\mu}\right.$ CC) interactions, and neutral-current neutrino ( $\nu$ NC) interactions respectively. These neutrino spectra are for the Bartol flux model, which produces neutrinos with a low energy limit of $200 \mathrm{MeV}$. For this reason, the charged-current interactions in figures 6.1 $\mathrm{a}$ and 6.1 b have visible neutrino energy spectra that are cut off at $200 \mathrm{MeV}$. The visible energy spectrum of neutral-current interactions (Figure 6.1 c) falls well below the $200 \mathrm{MeV}$ mark, as these interactions only deposit a fraction of the neutrino's energy in the detector.

The visible neutrino energy spectra in Figures 6.1 a - c are each shown for three separate subsets of the atmospheric neutrino flux: the solid line is for neutrinos that are predicted to interact in the detector, the dotted line is for neutrino interactions that would produce a trigger in the MINOS electronics, and the dashed line is for neutrinos that also pass the noise filter and reconstruct an event. The other Monte Carlo sets (B, $\mathrm{C}$, and D in Table 6.1) have some systematic variance from the primary set (A in Table 6.1). Figures 6.1 d, 6.1 e, and 6.1 f show the energy spectrum ratio of the three alternate $\mathrm{MC}$ sets to the primary set for the $\nu_{e} \mathrm{CC}, \nu_{\mu} \mathrm{CC}$, and $\nu \mathrm{NC}$ interactions respectively.

\subsubsection{Cosmic-Ray Muons}

A $1.84 \times 10^{7}$ sample of cosmic-ray muons required to go through the detector was generated to analyze the cosmic-ray background for this analysis. To scale the cosmic-ray muon simulated set to the data exposure, a comparison is made to the rates of stopping and through-going muons. The same method has been employed in the Cambridge charged-current atmospheric neutrino analysis. [93].

A containment region is defined as $0.5 \mathrm{~m}$ from any of the eight octagonal edges and

5 planes from the outer supermodule faces. A through-going muon is an event with a single track that has both its vertex and end fall outside of this containment region. 
A stopping muon has a vertex outside of the containment region and an end inside of this region. These two types of events are distinct, (i.e. the sets of stopping and through-going tracks will share no overlapping events).

(a) Reconstructed Through-Going Muons

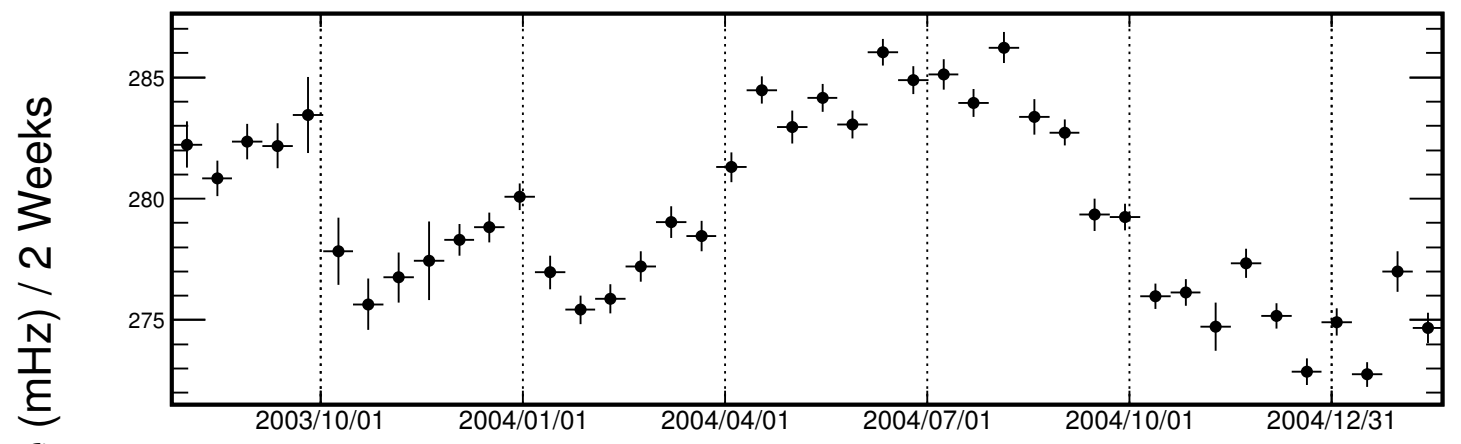

(b) Reconstructed Stopping Muons

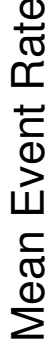

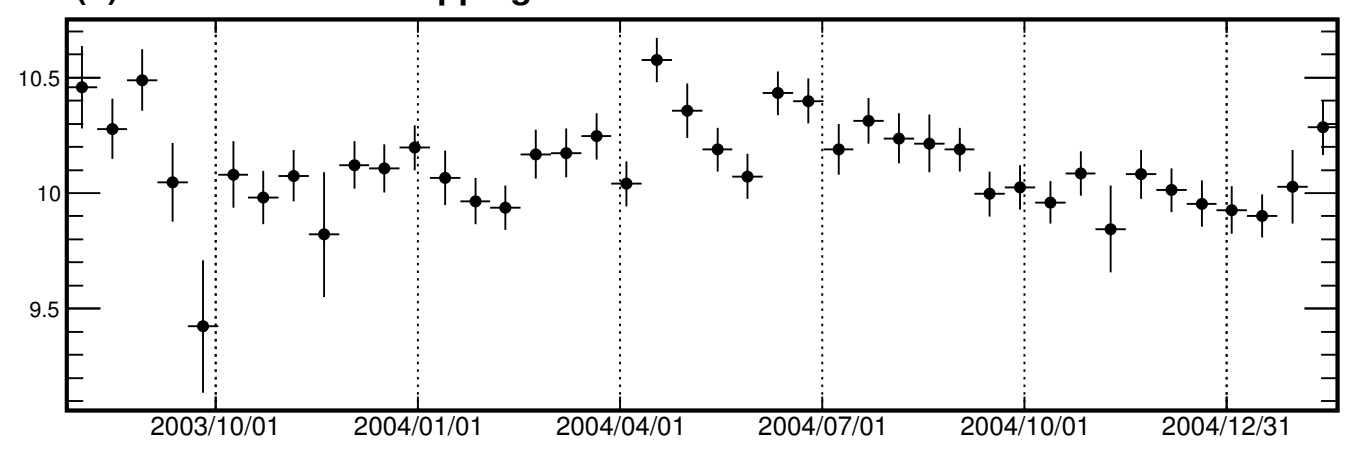

Figure 6.2: Rates of stopping and through-going muons, binned by fortnight.

In Figure 6.2, the rate of through-going and stopping muons per live-second is measured for each two week period in the data run. The average rate of the through-going muons equals 279. $\pm 4 \mathrm{mHz}$ and the average rate of stopping muons equals $10.1 \pm 0.2 \mathrm{mHz}$ Each of these averages is used independently to obtain the live-time-exposure equivalent for a $1 \times 10^{5}$ simulated cosmic-ray muon set, which is $34.5 \pm 0.5$ hours and $34.9 \pm 0.7$ hours for through-going and stopping muons respectively.

Used independently, the through-going and stopping muons effectively divide the normalizing sets into higher and lower energy cosmic rays. A muon loses energy as it passes through steel at a rate of $11.4 \mathrm{MeV} / \mathrm{cm}$, given that the muon is at the minimum ionizing energy [19]. For a muon to stop in the detector's fiducial region, the muon energy would have to be below an energy threshold which would depend on the entry 
angle $\left(E_{\mu}<9.6 \mathrm{GeV} / \cos \theta_{\text {zenith }}\right)$ [80]. A seasonal variation is seen in the through-going muon rate (Figure 6.2] a). This is less pronounced at lower muon energies [97, which explains the lack of a variation in the stopping muon rate (Figure 6.2 b).

An additional effect investigated is the impact the detector geometry and incident muon direction might have on the normalization. The through-going muons are divided into three categories: 1)side entry to side exit, 2)side entry to plane-face exit, and 3)plane-face entry to side exit. Similarly, the stopping muons are divided into two categories: side entry and plane-face entry. The variance in the flux normalization factor across these five sets is used to place a systematic error on the overall measurement.
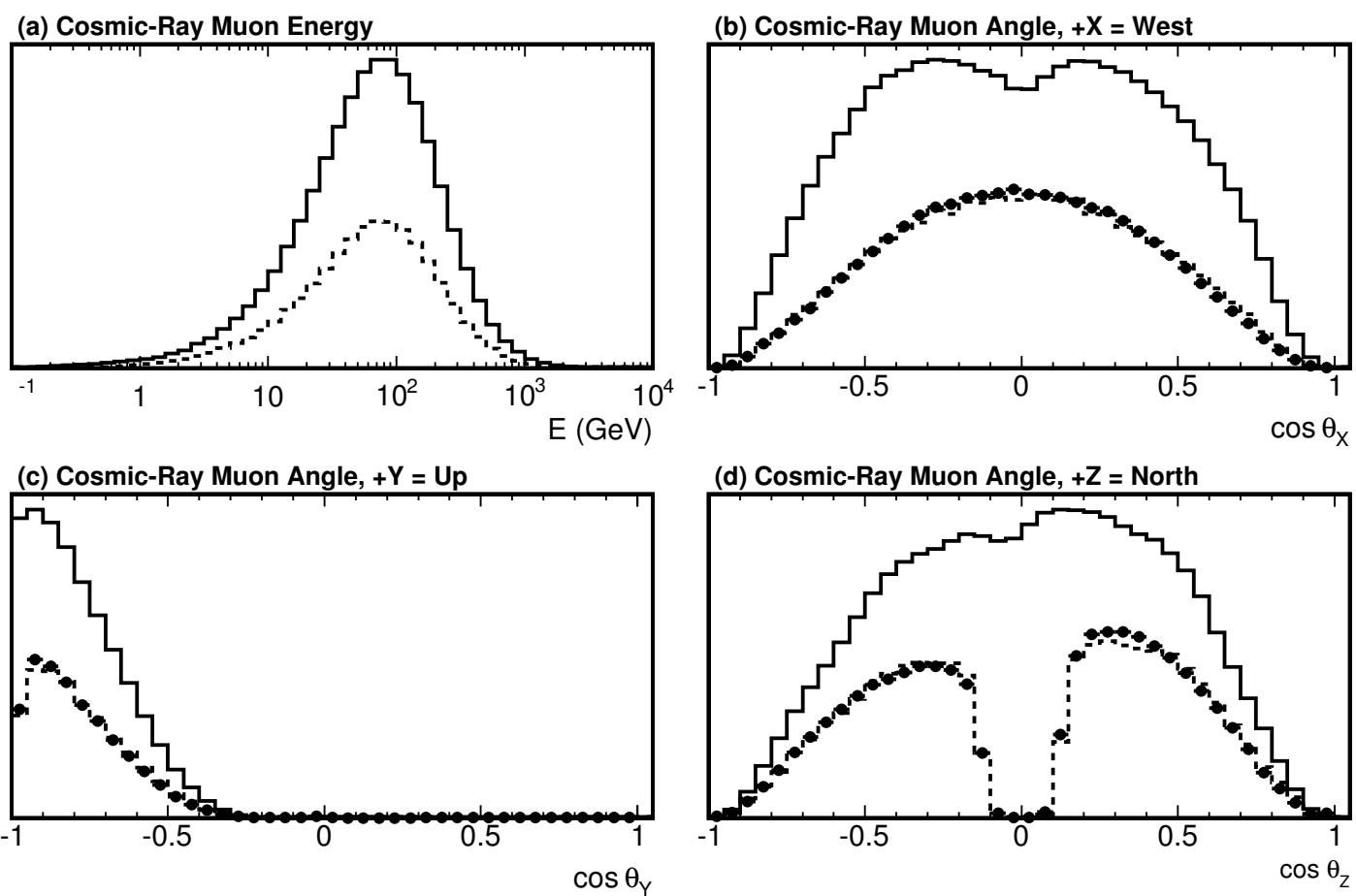

Figure 6.3: Spectra of Simulated cosmic-ray muons in the: a - true muon energy and $\mathrm{b}, \mathrm{c}$, and $\mathrm{d}-$ angular distributions in the $\mathrm{X}, \mathrm{Y}$, and $\mathrm{Z}$ detector coordinates. The three angular distributions also show data events with a single track. Spectra are for: solid line $=$ simulated muon sample, dashed line $=$ simulated muons with a single reconstructed track, and points for data events with a single track.

The full $1.84 \times 10^{7}$ simulated-muon sample is found to have an equivalent exposure of 267 live days. In order to scale to the data exposure, this set is multiplied by a factor of 
$1.586 \pm 0.019$ (stat.) \pm 0.065 (syst.). Figure 6.3 shows the true energy and angular spectra for these events, first at the base simulated flux (with selection selection requirements) and then for those events that have a single constructed track, as described in Section 5.4 The angular spectra also show the data events with a single constructed track overlaid on the MC. The energy spectra do not show overlaid data events, because high energy muons passing through the detector have no reliable method of resolving energy.

A Monte Carlo set consisting of $2 \times 10^{6}$ low energy cosmic-ray muons was generated with a constant energy distribution from 0 to $2 \mathrm{GeV}$ (energy at the detector) to investigate the appearance of lower-energy muons. The flux at can be extended from the flux model that was used for the $1.84 \times 10^{7}$ muon sample. The energy spectrum that was used to generate the $1.84 \times 10^{7}$ cosmic-ray muon set was cut off at $2 \mathrm{GeV}$, but the spectrum was extended down to $0 \mathrm{GeV}$ with an event distribution constant in energy. However, this is a small portion of the $1.84 \times 10^{7}$ cosmic-ray muon sample, and provides little information about events in the 0 to $2 \mathrm{GeV}$ energy range. The flux normalization from the $1.84 \times 10^{7}$ cosmic-ray muon sample can be extended to the 0 to $2 \mathrm{GeV}$ energy sub-set of the $2 \times 10^{6}$ cosmic-ray muon sample. Using this extended normalization, $2 \times 10^{6}$ cosmic-ray muon sample scales to the data exposure with a multiplicative factor of $0.259 \pm 0.004$ (stat.) \pm 0.011 (syst.).

\subsubsection{Rock Neutrons}

When a high energy cosmic-ray muon passes through the rock surrounding the detector, daughter particles from interactions with the rock material will follow the muon. If the muon passes through rock near the wall of the detector hall, one these daughter particles could reach the detector. A background to the neutrino signal occurs when such a neutron hits the detector and its parent muon misses the detector.

The simulation of such events is accomplished by taking a large number of muons, using the background muon flux from Section 6.3.2. These are passed through a large box around the detector that is composed of the space between the detector and the walls, and the rock around the detector. The Geant simulation tracks all resulting particles, including the high energy neutrons from muon interactions with the rock material. Only events where the neutron hits the detector and the muon misses the detector are kept. Complete details regarding this set are described in NuMI Note [98]. 
The neutrons were generated using muons with the same flux distribution as the set in Figure 6.3. The live-time scale derived in Section 6.3.2 can be used again, based on the size of the parent muon sample $\left(1.80 \times 10^{8}\right.$ muons $)$ used to generate the neutron sample. Thus, the $1.80 \times 10^{8}$ cosmic muons used to generate the 45,833 neutron sample correspond to a 38.25 kty exposure. The entire sample scales to the given data exposure by $0.1619 \pm 0.0019$ (stat.) \pm 0.0066 (syst.). Over the $6.19 \mathrm{kty}$ exposure, approximately 7,420 neutron interactions are expected to occur in the detector with no muon observed in the detector.

These events are not of significant concern when looking for muon-neutrino interactions. The selection process outline in Chapter 7 shows that no neutrons survive the track selection process. However, when searching for electron-neutrino interactions, there is a non-trivial background contribution from rock neutrons that this simulation set will be used to understand.

\subsection{Data Quality and Event Pre-Selection}

The 6.19 kty data sample consists of $2.2 \times 10^{7}$ reconstructed triggers. The simulated sets described in Section 6.3 are scaled to this data set based on the relative exposures, with expected counts shown in Table 6.2 The sum of the expected events is less than the number of observed data events by $\sim 3 \times 10^{6}$, a surplus of $\sim 20 \%$ in the observed count. Many of of these are low energy noise events caused by natural radiation in the mine, and are not simulated. Other events of concern are caused by triggers induced by hardware failures.

\begin{tabular}{|r|c|c|c|c|c|c|}
\hline & \multicolumn{5}{|c|}{ Monte Carlo } \\
& Data $\left(\times 10^{6}\right)$ & $\stackrel{(-)}{\nu}_{e} \mathrm{CC}$ & $\stackrel{(-)}{\nu}_{\mu} \mathrm{CC}$ & $\nu$ NC & $\mathrm{CR} \mu\left(\times 10^{6}\right)$ & $\mathrm{CR} \mathrm{N}$ \\
\hline Generated & & 419.2 & 797.1 & 500.0 & 29.5 & 7420 \\
Triggered & & 336.0 & 614.9 & 141.5 & 18.0 & 5390 \\
Reconstructed & 21.5 & 287.3 & 538.6 & 111.1 & 17.6 & 4980 \\
\hline efficiency & & $68.5 \%$ & $67.6 \%$ & $22.2 \%$ & $63.0 \%$ & $67.1 \%$ \\
\hline
\end{tabular}

Table 6.2: Expected event numbers of simulated sets, from generation to reconstruction. Efficiency is found by comparing generated sample size to reconstructed sample size. 


\subsubsection{Cambridge Filter}

The Cambridge reconstruction process includes a filter that both cleans up low energy noise and removes high energy cosmic-ray muons, before attempting event reconstruction. The aim of this filter is to reduce the processing time and the size of the resulting reconstructed data files.

\section{"Good Planes" Selection}

The detector trigger will register many events that are low energy noise, the bulk of which are removed at the NoiseFilter stage of the reconstruction. To additionally reduce the low energy noise background, the reconstruction defines a "good plane" as a plane with at least one hit with more than 2 pe. An event is removed from the sample if it does not have at least 5 "good planes" over the entire event, and two "good planes" in each view.

(a) All Good Planes

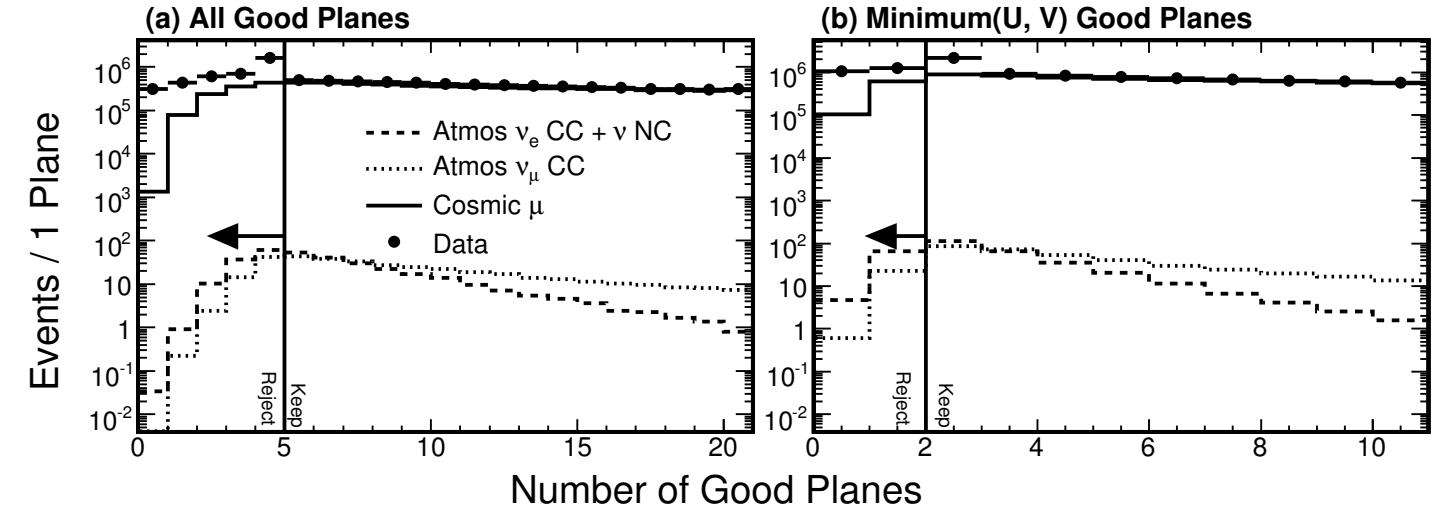

Figure 6.4: a - Number of total "Good Planes" in entire event and b - minimum "Good Planes" between views

The events removed in this selection process traverse very few planes, and do not deposit much energy in their passage. There is an obvious distortion near the selection threshold in Figure 6.4, where the observed data spectrum displays many more events than the expected cosmic-ray muon spectrum. The natural radiation in the mine causes these triggers, which are not simulated. 


\section{Fiducial Energy Containment}

The next stage of the Cambridge reconstruction filter reduces the large background of high-energy muons that pass from one edge of the detector to another (known as "through-going" muons). This type of event was used to normalize the simulated cosmicray muon flux in Section 6.3.2. All strips in an event are assigned a 3-D reconstructed position based at the charge-weighted-mean position of strips in adjacent planes with perpendicular views. If a plane doesn't have adequate information from the surrounding perpendicular-view planes, then is it excluded from this process.

Hits in the detector can then be separated into 11 regions for each supermodule: 2 regions for the outer 5 planes at both ends, 8 regions for the area made by measuring $30 \mathrm{~cm}$ in from each octagonal edge, and one more for the remaining inner portion of the detector. The accumulated pulse-height is tallied for the 22 total regions, and a region is determined to be "active" if the cumulative charge of the hits in that region is at least 5 photoelectrons (pe). The containment filter then defines the event as being in one of three classes:

- Fully Contained : No active outer edges

- Partially Contained : One active outer edge, or two adjoining active outer edges with the mean position of hits in those outer edge separated by no more than 1.0 $\mathrm{m}$

- Through Going : Two or more active edges that fail the partially contained criteria for two adjoining edges

An additional class that isn't listed above, but must be mentioned for completeness, is events which fail the above classification scheme. A possible situation that will cause this to happen is when the fiducially-contained region is not active with at least 5 pe. This case describes the bulk of the neutrinos that fail classification. Events with activity in both super-modules (multiple muons) will also fail this containment type classification. A multiple-muon event will not fail classification if all of the activity takes place in a single supermodule.

Only events which are defined as either fully-contained (FC) or partially-contained (PC) are selected. This reduces the largest single background of energetic through-going 


\begin{tabular}{|c|c|c|c|c|c|}
\hline \multirow[b]{2}{*}{ Containment Type } & \multirow[b]{2}{*}{ Data } & \multicolumn{4}{|c|}{ Monte Carlo } \\
\hline & & $\stackrel{(-)}{\nu}_{e} \mathrm{CC}$ & $\stackrel{(-)}{\nu}_{\mu} \mathrm{CC}$ & $\nu \mathrm{NC}$ & $\mathrm{CR} \mu$ \\
\hline Fail Classification & $8.57 \times 10^{5}$ & 8.91 & 18.49 & 2.58 & $7.68 \times 10^{5}$ \\
\hline Fully Contained & $1.50 \times 10^{6}$ & 118.20 & 254.69 & 44.14 & $1.32 \times 10^{6}$ \\
\hline Partially Contained & $3.48 \times 10^{6}$ & 30.22 & 139.16 & 13.18 & $3.06 \times 10^{6}$ \\
\hline Through Going & $1.19 \times 10^{7}$ & 1.13 & 13.25 & 0.77 & $1.14 \times 10^{7}$ \\
\hline
\end{tabular}

Table 6.3: Distribution of containment types.

muons, so that later selections can be optimized based on a strongly reduced set. In Table 6.3, the $\nu_{\mu}$ CC interaction sample is shown separately from the $\nu_{e} \mathrm{CC}+\nu \mathrm{NC}$ interactions.

While the $\nu_{e} \mathrm{CC}$ and $\nu \mathrm{NC}$ interactions fall primarily into the $\mathrm{FC}$ class of containment, the $\nu_{\mu} \mathrm{CC}$ interactions also have a significant fraction of $\mathrm{PC}$ events. The design of the detector requires a muon to travel further than an electron or hadron to deposit all of it's energy, and thus, more PC events are found among the $\nu_{\mu} \mathrm{CC}$ interactions. When the sample is divided into track-like and showering events in Chapter $\mathbf{7}$, the subset of showering events contains strictly FC events, but the subset of track-like events recovers the PC events with a separated selection process.

\subsubsection{Hardware Based Selections}

The primary concern regarding specific hardware issues, is the avoidance of events that appear to have some level of containment and could therefore end up in the final selected neutrino sample. For this reason, events are discarded if their trigger took place in the last $100 \mu \mathrm{s}$ of a time frame. When the time frame ends, the readout of a data event is cut off. If a significant portion of the event occurred after this cut-off, this could make an event seem contained. Also, an actual neutrino event that took place at the end of the time frame may lose hits and give a false energy measurement, potentially distorting spectra important in the study of neutrino oscillations. The time frame is 1 second wide, so the $100 \mu$ s stipulation causes a $0.01 \%$ loss of live-time, a number which is absorbed in the statistical uncertainty of the sample size.

The readout chips of the detector should be mostly operational when an event happens. The detector monitoring systems write out detector activity information for each chip. A chip with little or no activity is flagged as a "dead chip". A "busy chip" is 
defined as a chip that is in use $30 \mu$ s prior to the current event. An event is rejected if it has more than 20 chips that are dead or busy.

\section{Light-Injection Triggered Events}

In order to calibrate the detector response for light collection at the strip ends, a system was developed to inject light at one strip end and be read out at the other strip end. The light-injection(LI) system pulses light on a group of fibers with a light-emitting diode (LED), and the signal is triggered on the PMT attached to the opposing strip end. This pulsing is also performed in the opposite direction to resolve response at both strips ends.

When taking data at the far detector, the LI system will pulse the strips interspersed with the collection of candidate events. The events triggered by LI pulsing are identified by information from the summary blocks that is written into the data stream, and discarded before the event reconstruction to avoid contamination of an analytic data set. In spite of this precaution, some LI triggered events do occasionally remain in the data stream and appear as actual events. An isolated trigger PMT will give a timing estimate to an LI event. Events are rejected if the trigger PMT was pulsed more recently than $30 \mu$ s from the event trigger time. The pulsing occurs at a rate of $50 \mathrm{~Hz}$ throughout the detector during data collection, so a $30 \mu$ s window, occurring 50 times per second, only affects the live-time of the detector by $0.6 \%$.

The trigger PMT catches many of the remaining LI triggered events, but there is still a small contamination in the data sample caused by low powered LI, producing events that seemed to be contained. These event can occur when a set of flashing LEDs are temporarily under-powered, and thus don't produce enough light to cross the full strip and produce a signal in the LI trigger PMT. Low-powered LI pulsing events are identified by a large fraction of single-ended strips.

The fraction of single-ended strips is seen in Figure 6.5, where there is a surplus in events with a large fraction of single-ended strips in the data that is not represented in the MC muons or neutrinos. It may also be observed in Figure 6.5 that the data distribution is not well simulated by the cosmic-ray MC set. The problem may be that the strip efficiency is over-estimated in the detector simulation, causing an overall shift 


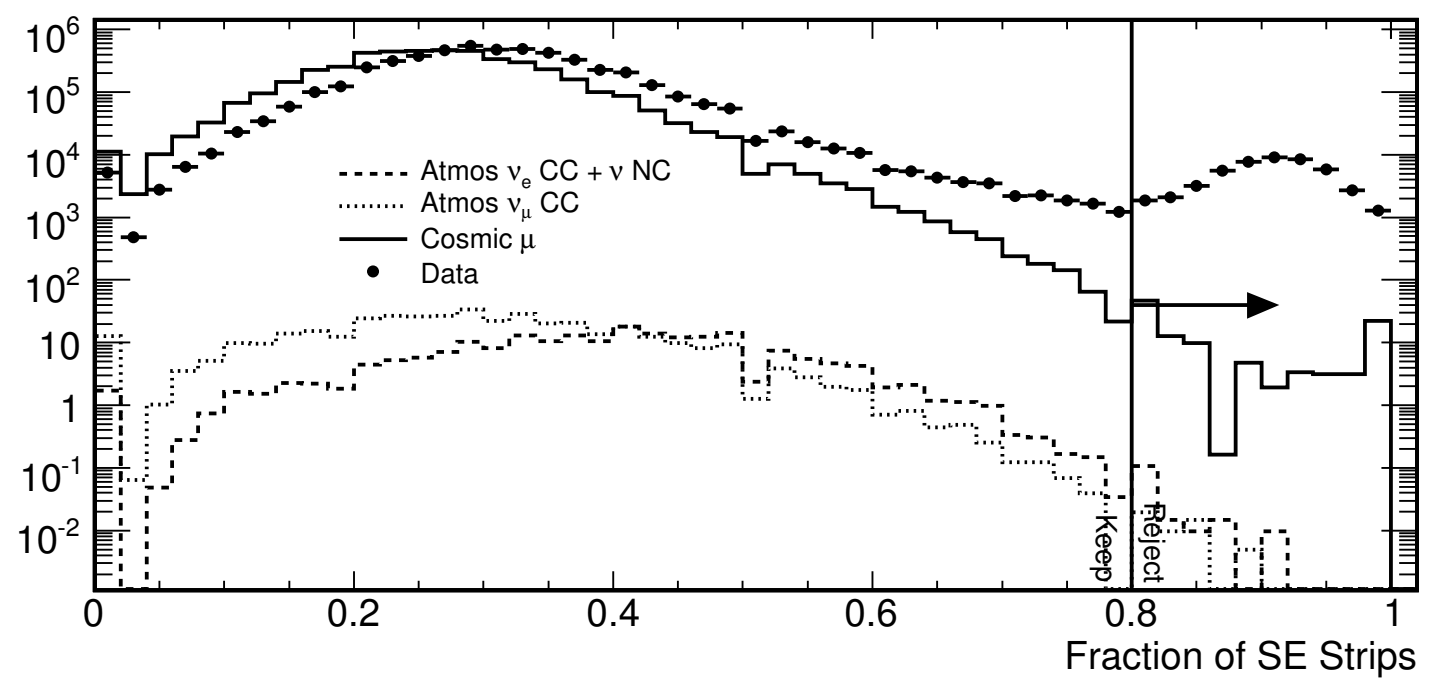

Figure 6.5: The fraction of strips in an event which have only a single end hit.

in the distribution. The selection requires that all events have no more than $80 \%$ singleended strips. The expected atmospheric neutrino spectrum does not change much near the point where the percent of single-ended strips is $80 \%$. For the expected count of all atmospheric neutrinos, only $\sim 5 \%$ have more than $60 \%$ single-ended strips, $<1 \%$ have more than $70 \%$ single-ended strips, and $<0.1 \%$ have more than $80 \%$ single-ended strips.

\subsection{Clean Analytic Set}

The results of the pre-selection are shown in Table 6.4. The top line shows the numbers of reconstructed events, which is the same as the last line of numbers in Table 6.2. The "good planes" selection removes a significant number of events from all of the categories. The containment selection reduces the cosmic-ray muon background by $\sim 25 \%$. The final line in Table 6.4 shows the overall selection efficiency, which is sufficiently higher for atmospheric-neutrino interactions.

Figure 6.6 shows the observed data event rate as a function of time throughout the pre-selection process. The full reconstructed set (Figure 6.6. a) has multiple spikes in the event rate, which are all quelled after the full set of pre-selection reductions is applied 
103

\begin{tabular}{|l|c|c|c|c|c|c|}
\hline & & \multicolumn{6}{|c|}{ Monte Carlo } \\
& Data $\left(\times 10^{6}\right)$ & $\stackrel{(-)}{\nu}_{e} \mathrm{CC}$ & $\stackrel{(-)}{\nu}_{\mu} \mathrm{CC}$ & $\nu \mathrm{NC}$ & $\mathrm{CR} \mu\left(\times 10^{6}\right)$ & $\mathrm{CR} N$ \\
\hline Reconstructed & 21.5 & 240.1 & 486.5 & 92.3 & 17.6 & 4980 \\
+ Good Planes & 17.7 & 158.4 & 425.6 & 60.7 & 16.5 & 4234 \\
+ Containment & 4.98 & 146.2 & 400.3 & 57.7 & 4.47 & 737. \\
+ Hardware & 4.97 & 146.2 & 400.3 & 57.7 & 4.47 & 737. \\
+Light Injection & 4.90 & 146.1 & 400.2 & 57.6 & 4.47 & 735. \\
\hline efficiency & $22.8 \%$ & $60.1 \%$ & $81.9 \%$ & $61.0 \%$ & $25.3 \%$ & $14.7 \%$ \\
\hline
\end{tabular}

Table 6.4: Observed and expected event numbers through pre-selection.

(Figure 6.6 d). The application of the selections to removed the low light LI pulsing events (Figures 6.6. $\mathrm{c}$ and 6.6. d) demonstrates that these types of events were prevalent during a few instances during the data run.

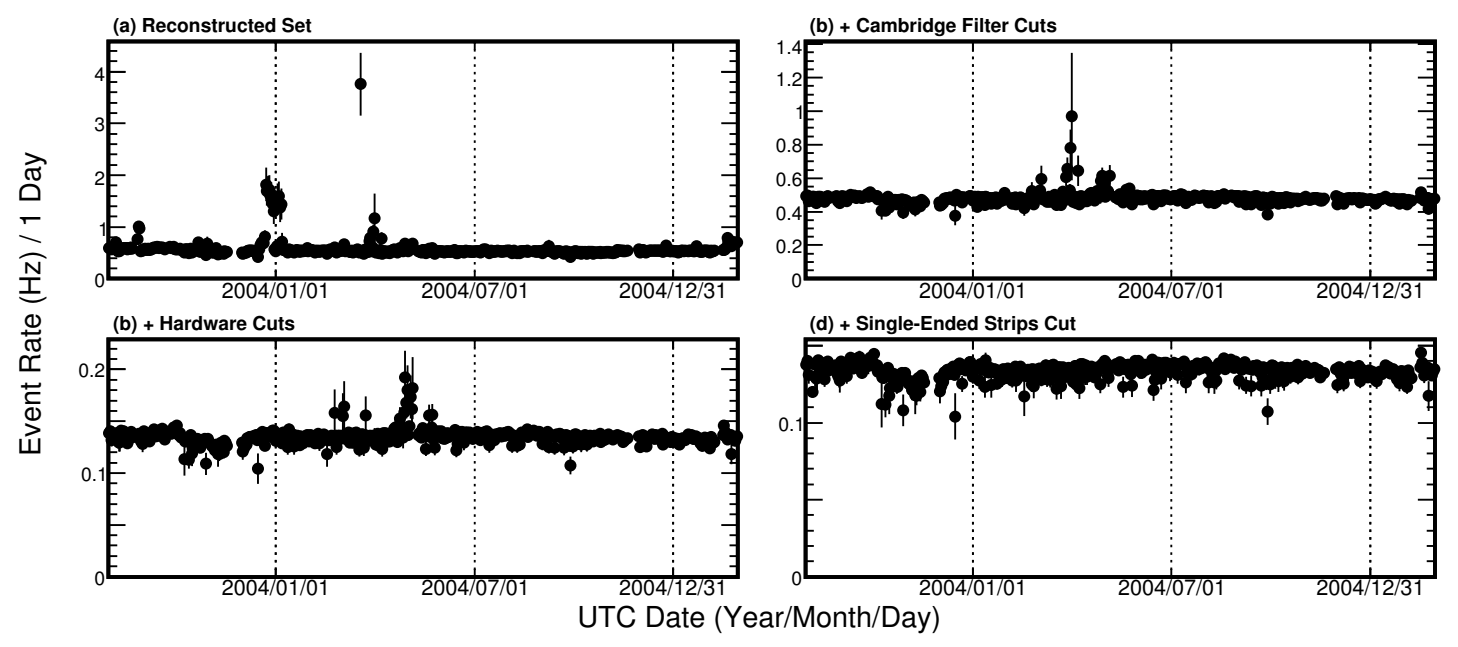

Figure 6.6: Data rate in the detector binned by day for all 418.5 live days after a sequence of selections: (a) primary data sample, (b) after the "good planes" selection, (c) containment and hardware selections, and (d) single-ended strips selection.

In Figure 6.7 the resulting visible neutrino energy spectra are shown from reconstructed to cleaned sets. Figure 6.7 a contains the spectra of neutrino interactions that produce showering events, including both $\nu_{e} \mathrm{CC}$ and $\nu \mathrm{NC}$ interactions. Figure 6.7,b contains the spectra of neutrino interactions that produces track-like events, which includes only $\nu_{\mu}$ CC interactions. The efficiencies in Tables 6.2 and 6.4 are consistently lower for showering neutrino interactions than for track-like interactions. The MINOS 
Far Detector was designed to optimally detect track-like neutrino interactions, and is expected to have lower efficiency when detecting showering interactions.

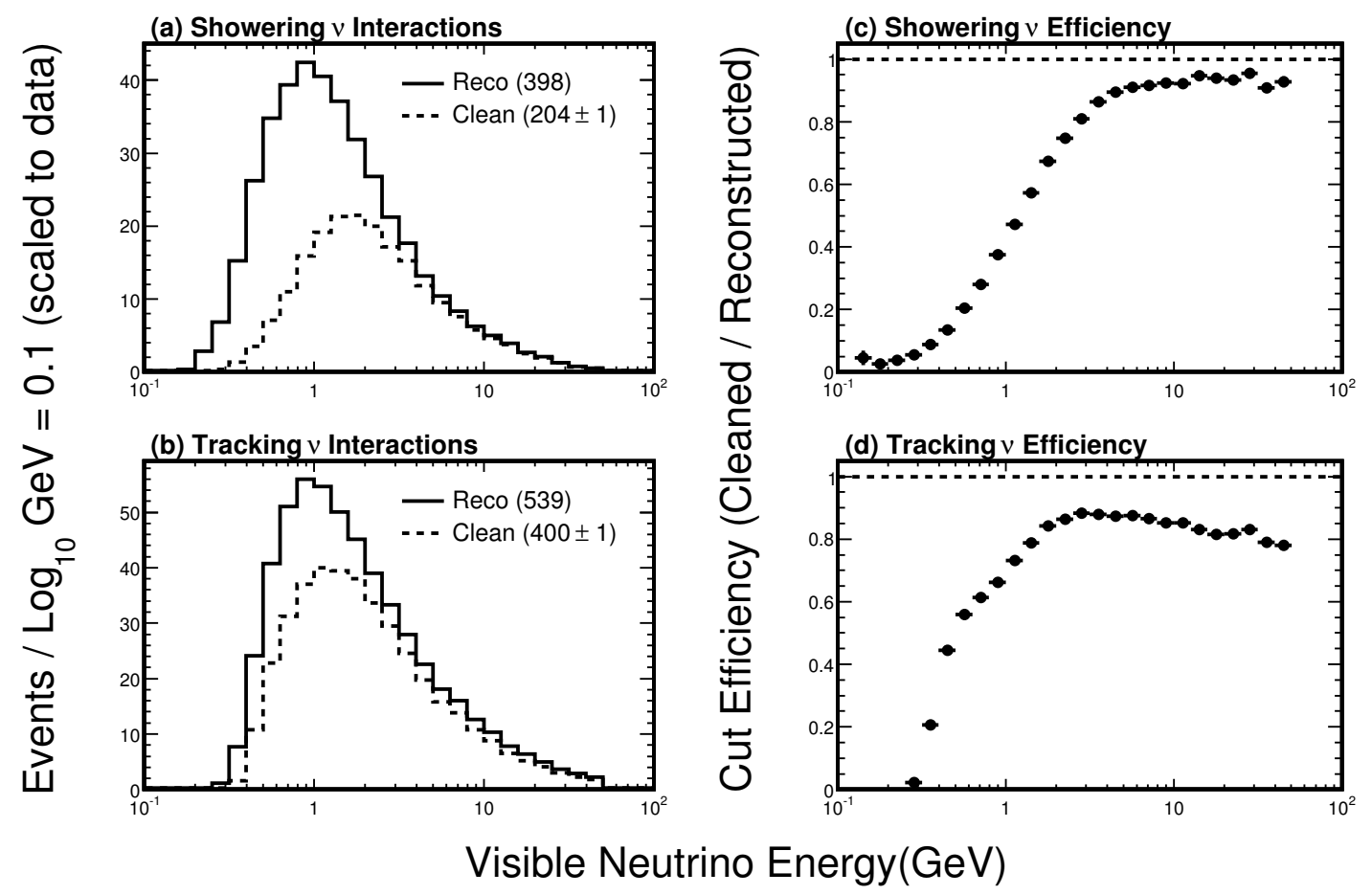

Figure 6.7: Neutrino energy deposited in the detector and efficiency of selections from reconstructed to cleaned set for $(\mathrm{a}, \mathrm{c})-$ showering $\left(\nu_{e} \mathrm{CC}\right.$ and $\left.\nu \mathrm{NC}\right)$ and $(\mathrm{b}, \mathrm{d})-$ track-like $\left(\nu_{\mu} C C\right)$ neutrino interactions

Figures 6.7] $\mathrm{c}$ and 6.7] show the efficiency of the pre-selection reductions relative to the reconstructed set for showering and tracking neutrino interactions. Both tracking and showering interactions have a low efficiency at low neutrino energy, but the showering neutrino interactions have a low efficiency that extends to higher energies. These pre-selection reductions are necessary to remove backgrounds that are difficult to distinguish from low-energy-neutrino interactions, due to both the abundance of the background events and the coarse-grained nature of the detector. The low efficiency of detection of low-energy-neutrino interactions is an unavoidable consequence of the configuration of the far detector.

After this initial data cleaning process, there is still a large background of cosmicray muons. The overall signal to background ratio for neutrinos to cosmic-ray muons is 
1:7400. The next step in the data selection process is to divide the data into showering and track-like sets, and to use containment and topological parameters to isolate two neutrino-rich data sets. 


\section{Chapter 7}

\section{Event Selection}

The cleaned data and Monte Carlo samples are culled for neutrino interaction candidates that can be analyzed in the context of neutrino oscillation. The current premise for atmospheric neutrino oscillation is that for the relevant propagation length and neutrino energy a muon neutrino may oscillate, but an electron neutrino will not. The neutralcurrent interaction rate for either muon or electron neutrinos will also not be affected by oscillation if the muon oscillates to a non-sterile neutrino. By virtue of their corresponding leptons, the difference between a muon and electron-neutrino charged-current interaction in a sampling hadron calorimeter is that a muon produces a track-like event while an electron produces a showering event. This difference is exploited in this chapter with a selection process to separate atmospheric neutrino candidates into track-like and showering events.

The cleaned set from Chapter [6] was divided into contained vertex track-like and showering sets. Then the showering and track-like event samples are culled based on event shape variables to maximally reduce the cosmic-ray muon background, and retain the atmospheric neutrino signal. Finally, the cosmic-ray veto shield is used to further reduce the cosmic-ray muon background for showering and well-defined downward going track-like events. 


\subsection{Track-like and Showering Sample Division}

The cleaned data and Monte Carlo samples are divided into sets of showering and tracklike events based on the Cambridge AtNuReco construction of a track or a shower (as described in Section [5.4). Figure 7.1 shows the distributions of the number of reconstructed tracks and showers for the data events along with the simulated cosmicray muon and atmospheric neutrino interactions. The events in this figure are those that remain after the selection process in Chapter [6. In all of the distributions shown in Figure 7.1 the track-like $\nu_{\mu} \mathrm{CC}$ interactions are separated from the showering $\nu_{e} \mathrm{CC}$ and $\nu \mathrm{NC}$ interactions.
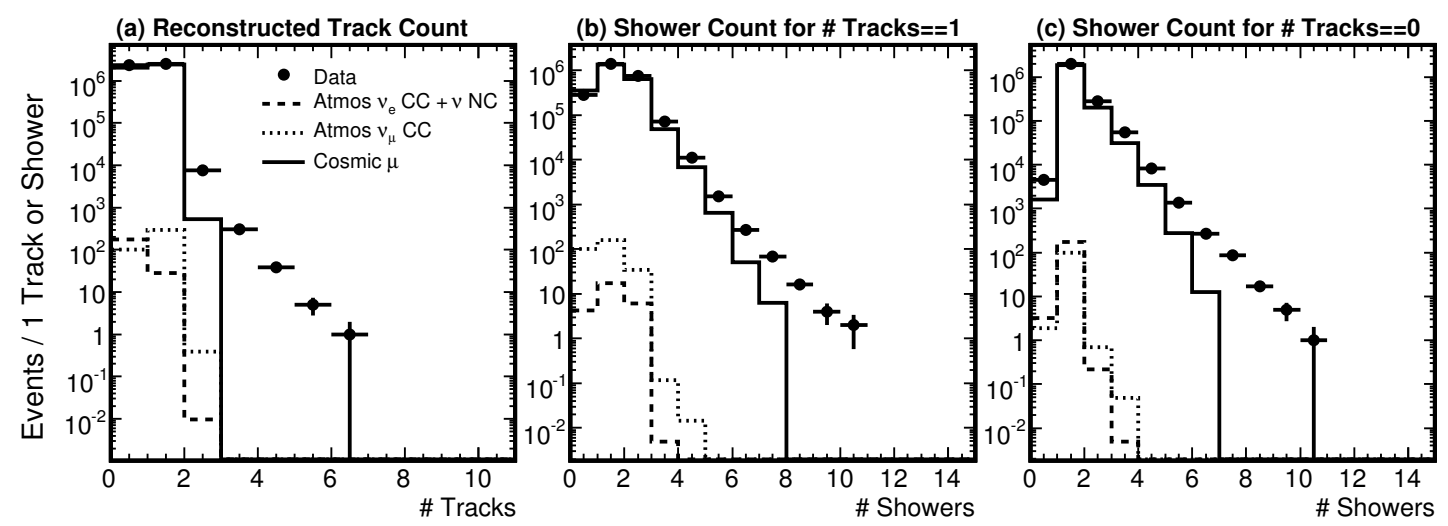

Figure 7.1: Numbers of reconstructed tracks or shower for: (a) total number of tracks, (b) number of showers with a single reconstructed track, and (c) number of showers with no reconstructed tracks.

The distributions in Figure 7.1 a demonstrate that $\nu_{\mu} \mathrm{CC}$ interactions are predominantly events that have a single track reconstructed. The $\nu_{\mu} \mathrm{CC}$ interactions that fail to reconstruct a track are mostly either a muon with a low incident angle relative to the steel plane face, or a low energy muon (either due to a low energy incident neutrino or a small fraction of the neutrino's energy being departed to its lepton). The track-like neutrino candidates are defined as those with one track and any number of showers.

The showering neutrino candidates have been defined as events that have no tracks and a single shower. Figure 7.1]c shows the number of showers reconstructed for events that have no tracks reconstructed. In addition to the removal of a large fraction of the $\nu_{\mu} \mathrm{CC}$ interaction, an immediate benefit of requiring that no tracks are reconstructed is 
that this set will have no overlap with the tracking set at the outset of the event selection. Of the $\nu_{e} \mathrm{CC}$ and $\nu \mathrm{NC}$ interactions, $16 \%$ have a reconstructed track. Many of these are events with a single hard pion that is difficult to distinguish from a short muon in the detector. This is an unfortunate loss, and a future direction for this analysis could be to add those events back in with an isolated selection. The single shower requirement retains showering neutrino interactions with a $98 \%$ efficiency in the showering neutrino candidate set.

\subsection{Vertex Containment}

The fiducial containment requirement in Section 6.4.1 deals only with energy deposited in the outer regions of the detector (up to $30 \mathrm{~cm}$ from an octagonal edge, up to 4 planes from a supermodule end). Under the energy containment requirement, an event must first be classified as FC or PC to be selected. The showering events have been selected exclusively as FC, while track-like events have been selected as either FC or PC. A reconstructed track and shower both have a vertex point, which is a 3D location in the detector from which the interaction most likely originated. This reconstructed vertex will have the same containment applied for both showering and track-like events to establish a consistent and comparable fiducial volume.

When a track is constructed, it is assigned a vertex (origin of the interacting particle), and an end (final rest point of the particle). A shower is constructed with only a vertex, as the diffuse nature of a shower makes the localization of an end point difficult. A containment criterion was developed based on the detector geometry, and applied to the track and shower vertices. Since tracks are constructed with an end, the containment

of the end point of a track is used to distinguish between the classes of fully-contained and partially-contained tracks.

\section{- Octagonal Vertex Depth -}

The distance from the event vertex to the nearest octagonal edge must be at least 50 $\mathrm{cm}$. The $50 \mathrm{~cm}$ window inward from the detector edge spans 10 strips. Given the average $3.57 \mathrm{~cm}$ gap between steel planes, the $50 \mathrm{~cm}$ window decreases the angle by which a muon can enter the detector undetected to $4^{\circ}$. This constraint on the event 
vertex location reduces the steel fiducial volume by $10 \%$.

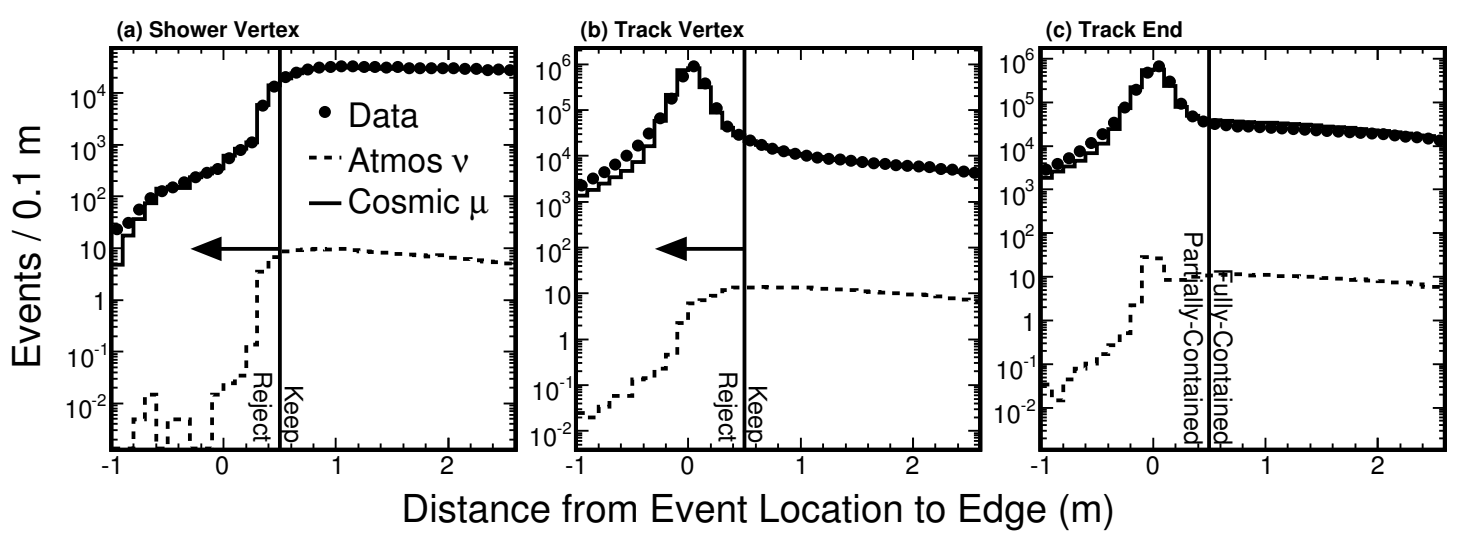

Figure 7.2: Distance from event location to the nearest detector edge for the: (a) shower vertex, (b) track vertex, and (c) track end

The shower vertex octagonal depth distributions are shown in Figure 7.2 a, and track vertex octagonal depth distributions in Figure 7.2. b. The cosmic-ray muon and data octagonal depth distributions are peaked at VtxDepth $\sim 0.0 \mathrm{~m}$ for the track-like events, but diminish rapidly for VtxDepth $<0.5 \mathrm{~m}$ for the showering events. The sample of showers is constrained to events which have been classified FC by energy containment. The sample of track-like events may be classified FC or PC by energy containment. The peak in the distribution of cosmic-ray-muon-induced track-like events at $0.0 \mathrm{~m}$ (the detector edge) is due to stopping muons that are permitted into the sample with the PC classified events from energy containment.

The track end in Figure $7.2 \mathrm{c}$ has the same feature at $0.0 \mathrm{~m}$ in the cosmic-ray muon distribution from the admission of the PC energy containment events. The track-end distribution of simulated atmospheric-neutrino-induced events has a peak around 0.0 $m$ that does not have an analogous feature in the track vertex distribution. This peak results from $\nu_{\mu} \mathrm{CC}$ interactions in which the muon exits the detector. The energy containment of PC classified events in the track sample was retained to save the $\nu_{\mu} \mathrm{CC}$ interactions with a muon that exits the detector. 


\section{- Nearest Supermodule Plane Edge -}

An event vertex is assigned an associated plane that correlates the nearest steel and scintillator plane to the event's vertex position ( 5 plane cut). This plane must be at least five planes from the nearest supermodule edge. The first supermodule spans planes 1 to 248, and the second supermodule spans planes 250 to 485 . The five plane requirement deems that the events vertex must fall in a plane range of $[6,243]$ or $[255$, 480].
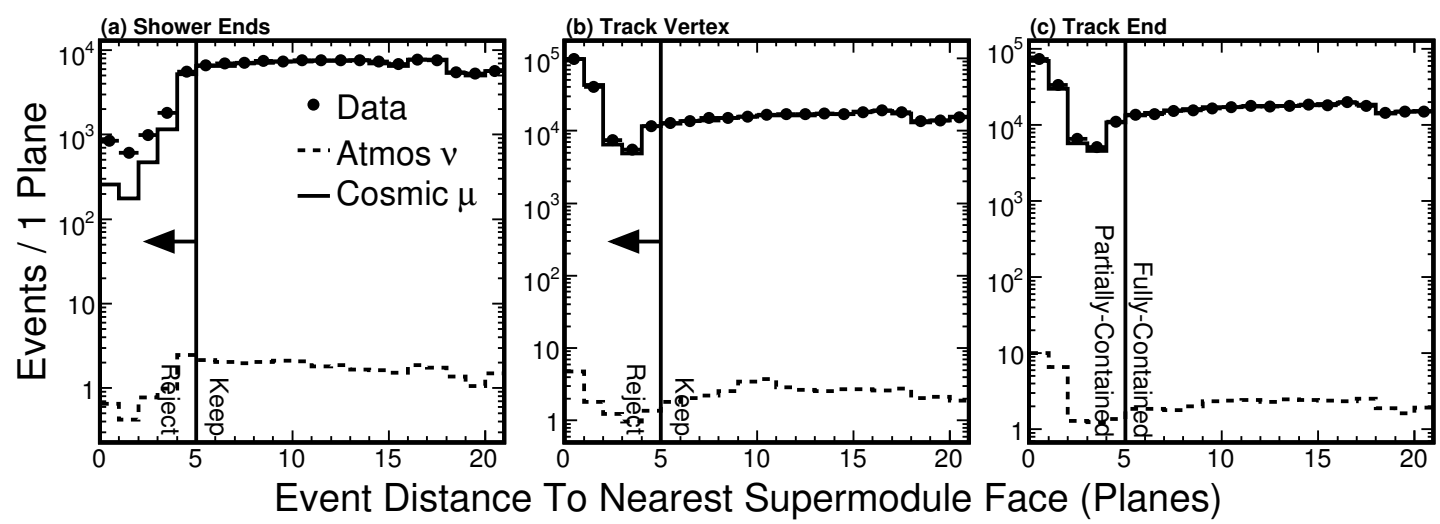

Figure 7.3: Distance from event location to the nearest supermodule plane face for: (a) shower end proximal to supermodule face, (b) track vertex, and (c) track end.

While a shower is only constructed with a single 3D vertex point, is is also constructed with a span in $\mathrm{Z}$ given by the first and last plane that has a hit associated with the shower. As discussed in section 5.4, the shower vertex is not easily found. The distance from the shower to the nearest supermodule plane face (Figure [7.3 a) is the minimum distance to the nearest supermodule plane's face between the first and last plane in the shower. This allays concerns presented by the bifurcated distribution from Figure 5.7

The 5 plane cut is required for the vertex of all tracks, as shown in Figure 7.3. b. The distribution of atmospheric neutrinos in Figure 7.3, b has a slight enhancement at zero. The tracks from simulated atmospheric neutrinos with a vertex that reconstructs so near a supermodule plane face are predominantly deep inelastic $\nu_{\mu} \mathrm{CC}$ interactions near the supermodule gap, where the muon continues into the other supermodule. The interaction point near the gap is dominated by the hadron shower that accompanies a 
deep inelastic scattering event and thus, the event reconstructs to a single shower on one side of the gap and a track on the other side, starting right at the plane edge.

The track-end distribution from Figure [7.3. c of simulated atmospheric neutrino events peaks beneath the 5 plane threshold, due to $\nu_{\mu} \mathrm{CC}$ interaction in which the muon exits the detector through a supermodule plane face. If the track end fails the 5 plane selection then the track may be classified as "partially-contained" by fiducial containment.

\section{- Coil Hole -}

The radial distance from the event vertex to the center of the detector must be at least $40 \mathrm{~cm}$ (coil hole cut) to avoid events entering through the hole for the magnetic coil in the center of the detector. The coil hole in the steel has a $20 \mathrm{~cm}$ radius. Instrumented strips start approximately $30 \mathrm{~cm}$ radially from the center of the detector.

The coil hole cut for showering events is based on the shower vertex (Figure [7.4 a), and for track-like events is based on the track vertex (Figure [7.4. b) and the track end (Figure 7.4. c). The subset of partially-contained track-like events does not include events that exit through the coil hole; these events are discarded instead. The coil hole cut removes a small portion of steel for potential neutrino interactions. The excluded ring is only $\sim 1 \%$ of the detector volume.

Cosmic-ray muons are more likely to enter through the coil hole on outer planes, and appear as contained vertex events, than they are on inner planes. To remove this background, an event with a vertex that is either at most 15 planes from one of the outside supermodule edges or at most 10 planes from one of the inside supermodule edges is required to occur at least $1.0 \mathrm{~m}$ radially from the center. This additional restriction is required of the shower vertex (Figure 17.4. d), track vertex (Figure 7.4. e), and the track end (Figure $\mathbf{7 . 4} \mathrm{f}$ ).

The vertex containment criterion differs from the energy containment requirement (Section 6.4.1). The vertex containment establishes a practical volume to which neutrino interactions are limited. The energy containment does not create a strict interaction region. The volume established by the vertex containment is the set of two octagonal volumes, with a hole in the center that is wider near the edges. The supermodules have 2.02 and 1.92 kiloton masses for supermodules one and two respectively. The combined 

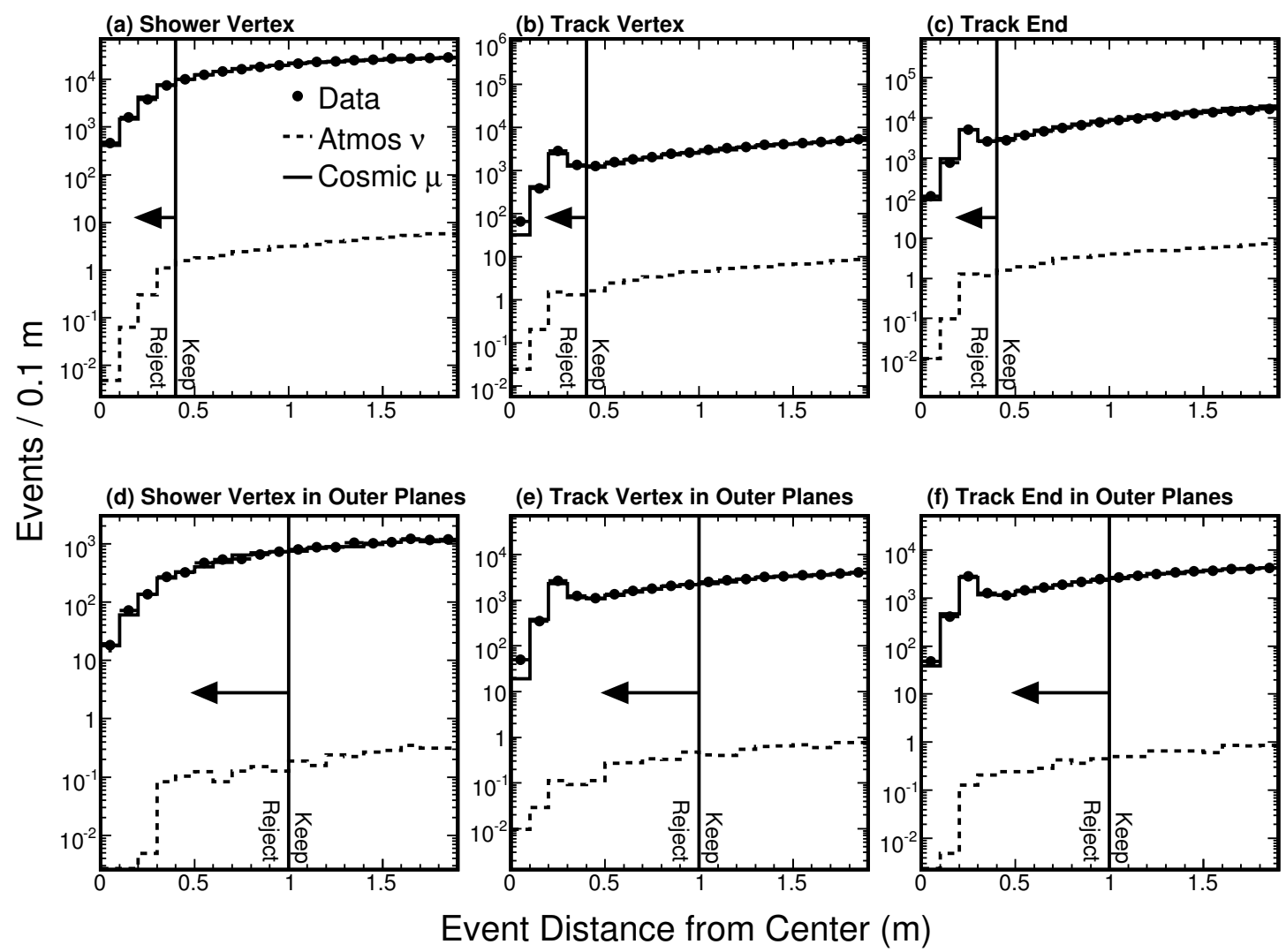

Figure 7.4: Radial distance from detector center to the event location for: (a) shower vertex, (b) track vertex, (c) track end, and $\mathrm{d} \leftrightarrow \mathrm{f}$ for the subset of events in which the relevant event location is 15 planes from the outer edge or 10 planes from the inner edge of a supermodule.

3.94 kiloton mass is $\sim 70 \%$ of the original 5.4 kiloton detector mass. Combining the vertex and energy containment restrictions, the full sets are reduced and divided into three sets: fully-contained showers, fully-contained tracks, and partially-contained tracks.

\subsection{Event Classification}

The Cambridge reconstruction filter identifies a containment type as one that is based on the energy deposited in regions of the detector, and classifies events as FC or PC. The sample of showering events will only use the FC events from the energy containment. The sample of track-like events however contains both the FC and PC from the energy 
containment. The vertex containment cut is applied to the showering and track-like event sets, and the events are divided into four classes:

- FC Shower - 1 Shower, No Tracks, Filter Classified FC, Vertex Contained

- FC Track - 1 Track, Filter Classified FC, Vertex and End Contained

- PCDN Track - 1 Track, Filter Classified FC or PC, Vertex Contained, End Not Contained, Vertex $\cos \theta_{Y}<0, V t x_{Y}>E n d_{Y}$

- PCUP Track - 1 Track, Filter Classified FC or PC, Vertex Contained, End Not Contained, Vertex $\cos \theta_{Y}>0, V t x_{Y}<E n d_{Y}$

The partially contained track-like events are divided into two subsets (upward and downward going tracks). This is done because the primary background to downward going tracks is from cosmic-ray muons with a small zenith angle, and the primary background to upward going tracks is from cosmic-ray muons that reconstruct in the wrong direction. The expected signal and background sizes for each class are shown in Table [7.1]

\begin{tabular}{|c|c|c|c|c|c|c|}
\hline & & \multicolumn{5}{|c|}{ Monte Carlo } \\
& Data & $\stackrel{(-)}{\nu}_{e} \mathrm{CC}$ & $\stackrel{(-)}{\nu}_{\mu} \mathrm{CC}$ & $\nu \mathrm{NC}$ & $\mathrm{CR} \mu$ & $\mathrm{CR} \mathrm{N}$ \\
\hline Shower Set & $2.00 \times 10^{6}$ & 128.1 & 97.5 & 44.5 & $1.83 \times 10^{6}$ & 412.4 \\
\hline Containment & 794772 & 92.4 & 61.1 & 31.3 & 767913 & 73.6 \\
\hline \hline Track Set & $2.55 \times 10^{6}$ & 16.0 & 299.7 & 11.8 & $2.40 \times 10^{6}$ & 260.4 \\
\hline Contained Vertex & 86959 & 11.99 & 231.4 & 8.71 & 76264. & 17.9 \\
\hline FC Track & 40177 & 10.54 & 153.4 & 7.50 & 36042. & 2.4 \\
PCDN Track & 41777 & 0.749 & 39.5 & 0.665 & 37099 & 8.26 \\
PCUP Track & 5005 & 0.700 & 39.4 & 0.573 & 5426.2 & 7.2 \\
\hline
\end{tabular}

Table 7.1: Sample sizes for divided sets.

The neutron contribution to the showering events is much more significant than to the track-like events. However, in both cases the expected neutron background is reduced significantly as a result of this initial containment selection. It was observed in the Soudan2 detector [99] that the neutron background decreases rapidly relative to the event vertex depth, which is consistent with the reduction observed with the containment cuts. 
Another quantity that merits mention is the ratio of oscillation sensitive neutrino interactions to all neutrino interactions (number of $\nu_{\mu} \mathrm{CC}$ : number of all other $\nu$ interactions) which, for the divided sets, is: 8:1 for fully-contained tracks, 27:1 for partiallycontained tracks, and 1:2 for fully-contained showers. The showering set still has a large enough expected contribution from oscillation-sensitive-neutrino interactions, that using this set for oscillation independent flux normalization would be problematic. The next step is to further reduce the background of the individual set by selecting events based on topological quantities to achieve separation of the atmospheric neutrino signal and the cosmic-ray background.

\subsection{Shower Selection}

A showering event is defined as an event that passes the data quality requirements and pre-selection (Section 6.4), has a single shower with no accompanying tracks (Section 7.1), and is defined as fully-contained by energy containment. The number of events that fall into this category for the simulated and observed samples are shown in the first line of Table 7.4. The values on the second line in this table are obtained by constraining the location of the shower vertex (Section [7.2).

\begin{tabular}{|c|c|c|c|c|c|c|}
\hline & \multicolumn{6}{|c|}{ Monte Carlo } \\
& Data & $\stackrel{(-)}{\nu}_{e} \mathrm{CC}$ & $\stackrel{(-)}{\nu}_{\mu} \mathrm{CC}$ & $\nu$ NC & $\mathrm{CR} \mu$ & $\mathrm{CR} N$ \\
\hline Pre-Selected FC Showers & 852658 & 102.2 & 67.1 & 34.5 & 823930 & 89.9 \\
+Vertex Containment & 794772 & 92.4 & 61.1 & 31.3 & 767913 & 73.7 \\
\hline
\end{tabular}

Table 7.2: Counts for the initial fully-contained showering event set.

At this point in the selection process, the signal to background ratio for neutrino selection in the showering sample is 1:4200. The remaining cosmic-ray muons have faked containment by entering the detector at a steep angle, and scattering on a steel plane in the magnetic field to change direction to appear as events that were initiated inside of the detector. To isolate neutrino interactions from the cosmic-ray muons in the showering set, the following progression of event selection is used:

1. Event Quality - Isolate higher quality events based on event characteristics.

2. Shower Quality - Isolate higher quality showers based on shower characteristics. 
3. Shower Trace - Use the direction and depth of the shower to make a modified vertex containment selection.

4. Shower Shape - Use the pattern of energy deposition and strip progression to identify the most neutrino-like events.

\subsubsection{Event Quality}

\section{- Single Supermodule Fiducial Containment -}

The showering event set has been constrained with fiducial containment of both hit energy (Section 6.4.1) and event vertex (Section 17.2) such that all events are fullycontained. In addition, a showering event is also required to occur in a single supermodule. This is accomplished by first requiring that all hits associated with the shower must be in a single supermodule. Second, $99 \%$ of the total event pulse height must be in the fiducial volume (3.7 m, 4 planes from edge) of the supermodule that contains the shower.
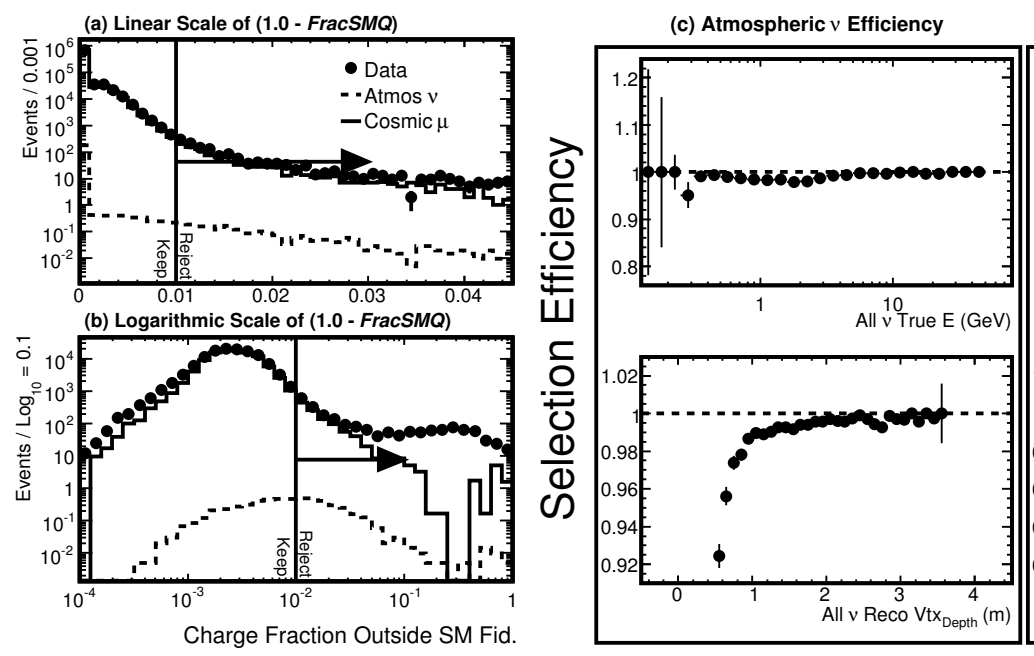

(d) Cosmic-Ray $\mu$ Efficiency

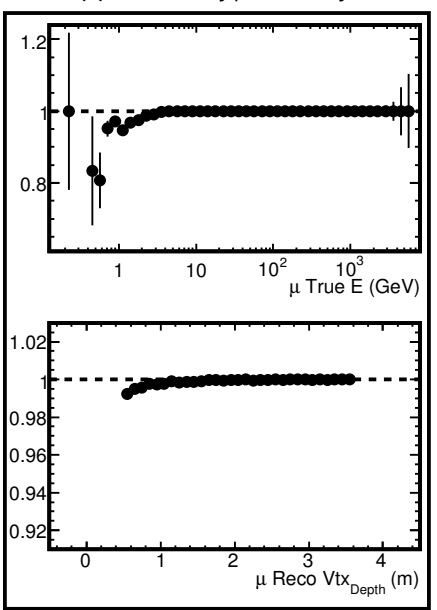

Figure 7.5: Fraction of energy located outside of the fiducial volume of the supermodule containing the shower.

Figure 7.5 shows the fraction of hit energy that is located in a region other than the fiducial volume (as defined by energy containment) that contains the shower, i.e. FracSMQ $=$ QSMShw/QTotal. This fraction is shown on a linear scale (Figure 7.5 a) and 
a logarithmic scale (Figure [7.5 b). The distortion between the expected and observed spectra is best seen in the logarithmic scale where $\operatorname{FracSMQ}>10^{-1}$.

The observed events with FracSMQ $>10^{-1}$ have been scanned by eye, and found to be predominantly of three pathologies: in order of precedence, 1)multi-muon activity, where the energy deposited in the opposing supermodule was not reconstructed to a track or shower, 2)low energy event near the detector edge, or 3)mis-reconstruction of through-going muons due to unresponsive strips. The efficiency in Figure 7.5. c is profiled against the spectrum of neutrino interaction depth. The neutrino interactions that fail this containment selection are predominantly near the edge of the detector. Showering neutrino interactions that are near the edge of the detector have a lower energy resolution because there is a possibility that a non-trivial fraction of the showering particles could escape between planes.

\section{- "Clean Planes" -}

The Cambridge filter (applied before reconstruction) culls low energy events by requiring a minimum number of "good planes" (see Figure 6.4). A "good plane" has one or more strips with at least 2 photoelectrons (pe). The showering events are more sensitive than the track-like events to low energy background, and so the "good planes" cut is extended by defining a "clean plane" similar to a "good plane". A "clean plane" must have one or more double-ended hits with at least 2 pe, rather than just any hit with at least 2 pe in a "good plane".

The same selection is made with "clean planes" as with "good planes". Showering events are required to have at least five overall "clean planes" (Figure [7.6 a) and two in each view (Figure 17.6 b). The "clean planes" cut significantly reduces the size of the expected showering neutrino sample. Figure [7.6]c shows the "clean planes" cut efficiency for the visible neutrino energy spectrum, where is it clear that this selection favors neutrino interactions with higher visible energies. The thickness of the steel planes in the far detector makes it difficult to differentiate low energy showering neutrino interactions from background radiation.

The reduction of the low energy $\nu_{e}$ CC interaction is detrimental to the electron neutrino analysis of sub-dominant oscillation. This particular analysis will not be considered in this thesis. For the purpose of normalizing the flux model, the reduction 

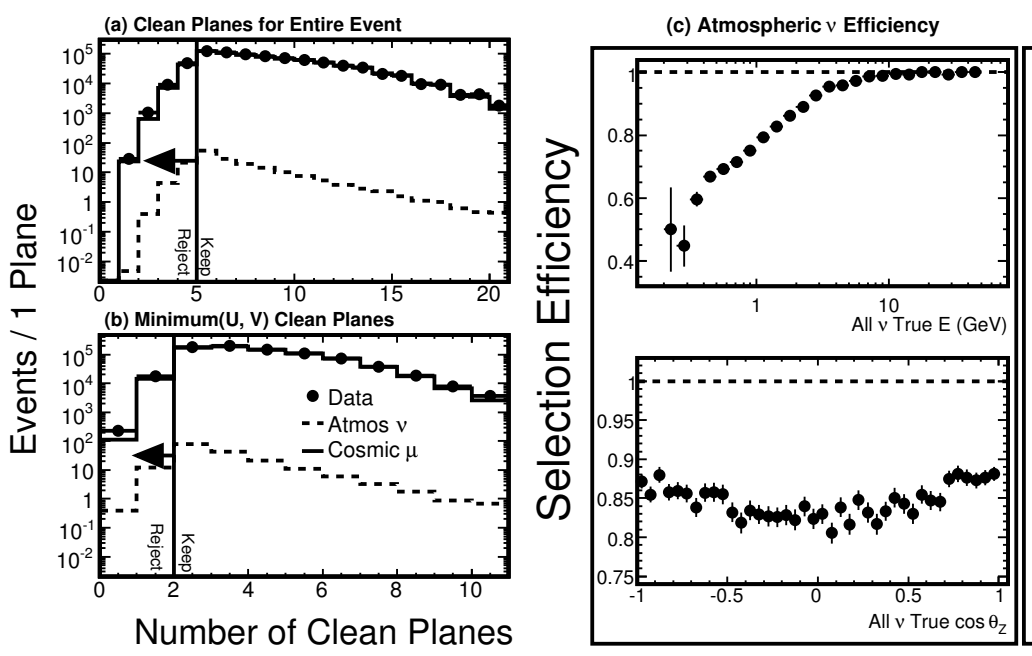

(d) Cosmic-Ray $\mu$ Efficiency

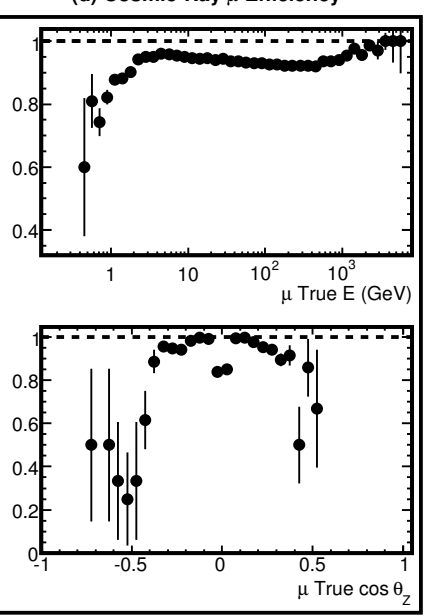

Figure 7.6: Number of "Clean planes" (one or more double-ended strips with at least 2pe) in showering events: (a) Total for all planes, and (b) minimum between U and $\mathrm{V}$-view planes.

of low energy $\nu_{e} \mathrm{CC}$ interactions is not as damaging. Any future analysis that seeks to use the $\nu_{e} \mathrm{CC}$ interactions to make measurements of sub-dominant oscillation will have to resolve the low energy backgrounds, while retaining the low energy neutrino interactions.

\section{- Strips - Planes -}

When a track-like event traverses few planes, and scatters enough for the hits to no longer appear to be on a straight line, the generation of tracks and showers (Section 5.4) will tend to identify this event with a shower rather than a track. The mis-classification introduces a non-trivial number of low energy $\nu_{\mu} \mathrm{CC}$ interactions into the showering sample. To reduce this background of oscillation sensitive neutrino interactions, a count is made of the number of active strips in the event and the total number of planes with at least one active strip. A showering event is required to have at least two more active strips than active planes.

Figure [7.7 a shows the number of active strips minus the number of planes with at least one active strip, a quantity which can be no less than 2. This selection reduces the contribution of $\nu_{\mu} \mathrm{CC}$ interaction to the showering sample by $13 \%$, while only 
(a) Active Strips - Active Planes

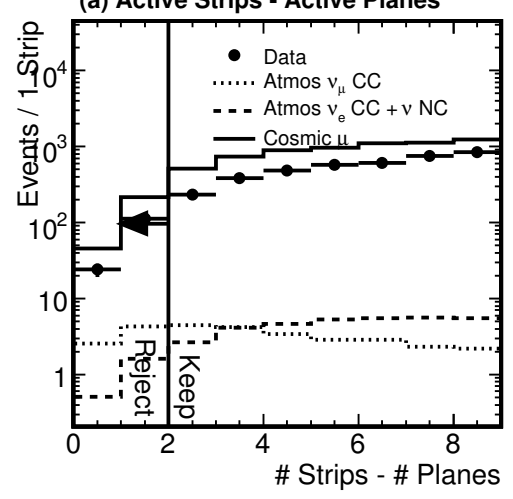

(b) Atmospheric $v$ Efficiency

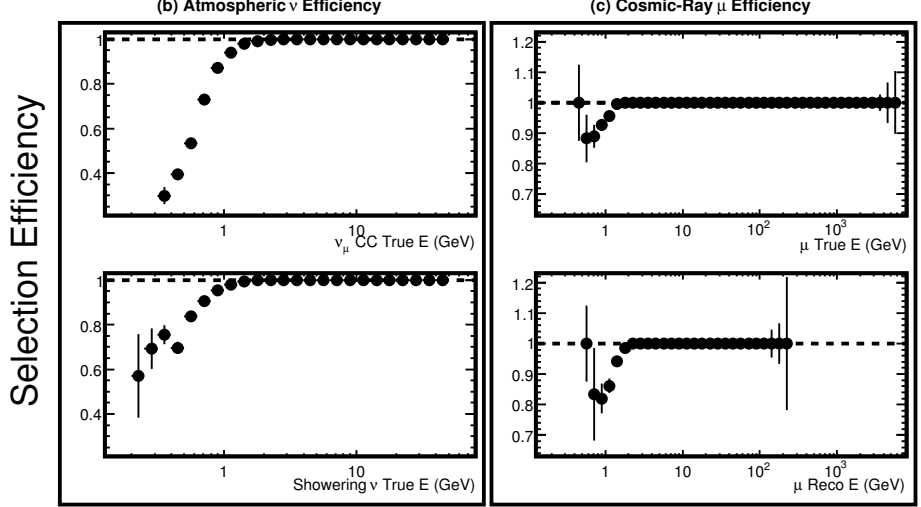

Figure 7.7: Number of active strips minus the number of active planes for showering events. There must be at least 2 more strips than planes in showering events.

reducing the contribution of $\nu_{e} \mathrm{CC}$ interactions by $1.5 \%$. Figures [7.7 b and 7.7]c show the efficiency of this cut as a function of the neutrino energy for $\nu_{\mu} \mathrm{CC}$ and $\nu_{e} \mathrm{CC}$ interactions respectively.

\section{- Maximum Plane Charge -}

A steep cosmic-ray muon that appears to have a contained vertex will, in many cases, deposit a large amount of energy in a single plane. To identify such events, the maximum amount of energy deposited in a single plane is shown in Figure 7.8 a, and requires that no plane in an event have 500 pe or more.

(a) Max. Single Plane Charge

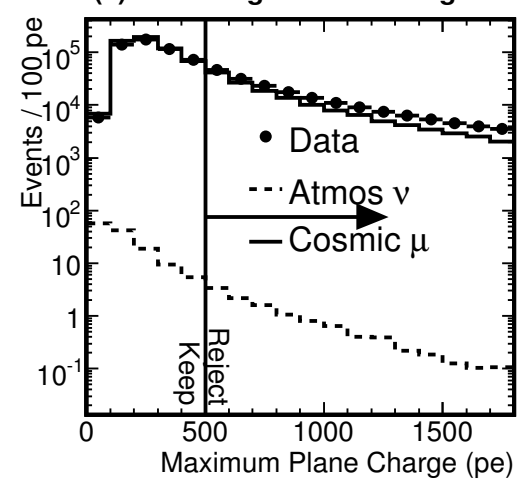

(b) Atmospheric $v$ Efficiency

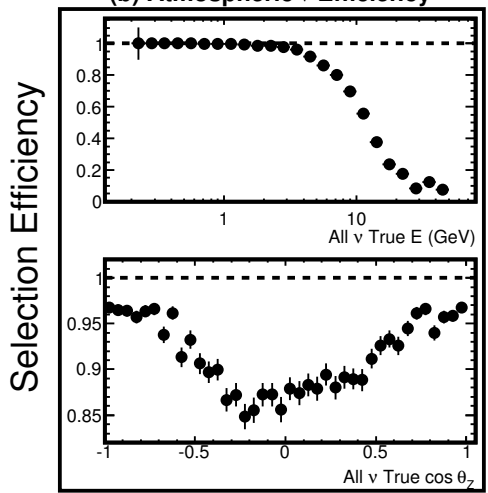

(c) Cosmic-Ray $\mu$ Efficiency

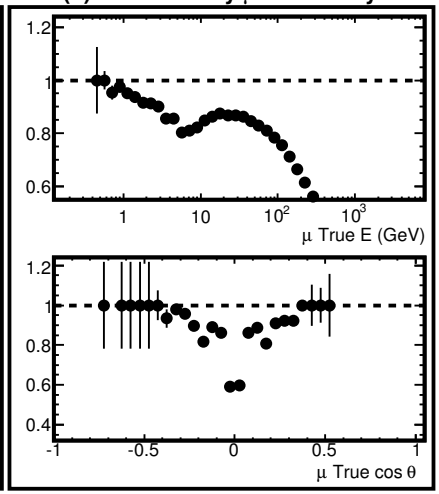

Figure 7.8: Maximum energy deposited in a single plane, discard events that have $\geq 500$ pe. 
The selection based on the maximum charge in a single plane will have a low efficiency to select neutrino interactions with high visible energy (Figure [7.8, b). Interactions from neutrinos that enter the detector at a small angle relative to the plane face ( $\cos \theta_{Z} \sim 0$ ) would deposit much of their energy in a single plane. The efficiency in Figure [7.8. c is lower at $\left|\cos \theta_{Z}\right| \sim 0$ than at $\left|\cos \theta_{Z}\right| \sim 1$.

\section{- Event Quality Selection Result -}

\begin{tabular}{|c|c|c|c|c|c|c|}
\hline & \multicolumn{6}{|c|}{ Monte Carlo } \\
& Data & $\stackrel{(-)}{\nu}{ }_{e} \mathrm{CC}$ & $\stackrel{(-)}{\nu}_{\mu} \mathrm{CC}$ & $\nu$ NC & $\mathrm{CR} \mu$ & $\mathrm{CR} N$ \\
\hline Contained Shower & 794772 & 92.4 & 61.1 & 31.3 & 767913 & 73.7 \\
+Single SM Contained & 792666 & 91.3 & 60.3 & 30.8 & 767032 & 68.8 \\
+ Clean Planes & 733922 & 78.0 & 51.2 & 25.5 & 715906 & 57.0 \\
+ Strips - Planes & 733786 & 76.8 & 44.3 & 24.5 & 715762 & 56.5 \\
+ Max Plane Charge & 512482 & 69.2 & 42.0 & 22.9 & 550653 & 40.6 \\
\hline Efficiency & $64 \%$ & $75 \%$ & $69 \%$ & $73 \%$ & $72 \%$ & $55 \%$ \\
\hline
\end{tabular}

Table 7.3: Number of events through event quality selection.

\subsubsection{Shower Quality}

The showering sample requires a single constructed shower, and this next set of selections is based on the shower quality to avoid optimizing selections on poorly reconstructed showers. Figure [7.9] a shows the distributions of the plane counts associated with the shower. There are few atmospheric neutrino interaction showers with less than 5 associated planes, and so showers are required be at least 5 planes long. There are a large number of cosmic-ray muon that have less than 5 planes. These cosmic-ray muons predominantly enter the detector at a small angle to the plane face, and exit the detector after traveling just a few planes.

The "plane completeness" of the shower is expressed as the fraction of the total active planes in an event that have a hit that is associated with the shower. The distributions of this fraction are shown in Figure [7.9] b. At least half of the active planes in the event are required to be associated with the shower.

The spectrum of observed data events in Figure [7.9] b diverges from the expected spectra below $30 \%$ of planes in the shower. This small fraction of the data set is due to 

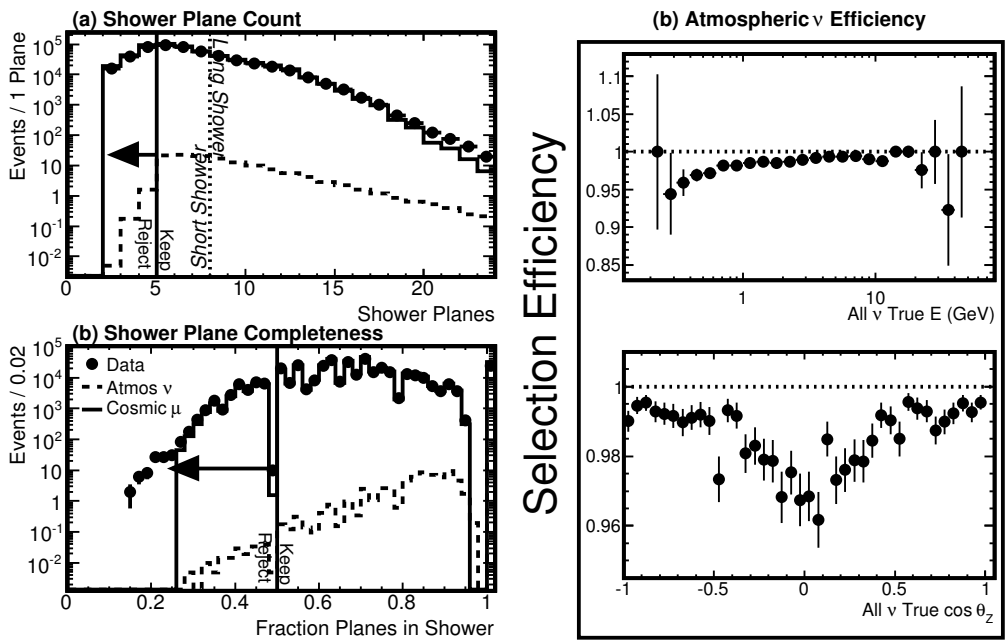

(c) Cosmic-Ray $\mu$ Efficiency

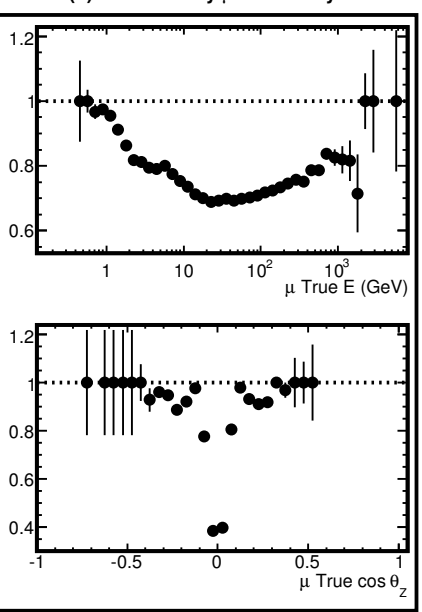

Figure 7.9: Number of active planes in the shower.

multiple cosmic-ray muons (which is not simulated). The requirement that a showering event is contained in a single supermodule eliminated many multiple-muon events, but some are contained in a single supermodule. These are reduced by requiring that at least $50 \%$ of active planes in a showering event are associated with the shower.

The remaining selection of showering events is performed by examining shower topological parameters. To optimize these selections, they will be divided into two classes based on the number of planes in the shower. Showering events with a shower that has 8 planes or less are considered "short shower" events. Events with a shower that has more than 8 planes are considered "long shower" events. 8 planes are chosen because electronics are grouped in the detector by 4 planes, and because using 8 planes divides the sample size approximately in half.

\subsubsection{Shower Trace}

The "event trace" refers to the distance from the event vertex to the nearest detector edge, traced back along the event direction. This gives the linear distance from the vertex to the location where the event would have entered the detector in order to create the event. The TraceZ, takes just the Z-Component of this trace distance, thus giving a measurement of how much steel the particle would have passed through to arrive at a location with a given direction. 
The direction of a shower is determined by using a 2D charge-weighted fit of shower hits in each view. The $2 \mathrm{D}$ fits from each view are combined to form a single $3 \mathrm{D}$ direction. The timing of these hits is used to determine if the direction is forward or backward relative the plane sequence. Most showers are too short to use hit timing to confidently assign a parity of the direction shower. To resolve this, two separate trace values are calculated. TraceZForward is the trace found by following the shower direction, given its current parity to nearest detector edge. The TraceZReverse is analogous to the TraceZ Forward, with the direction parity reversed.
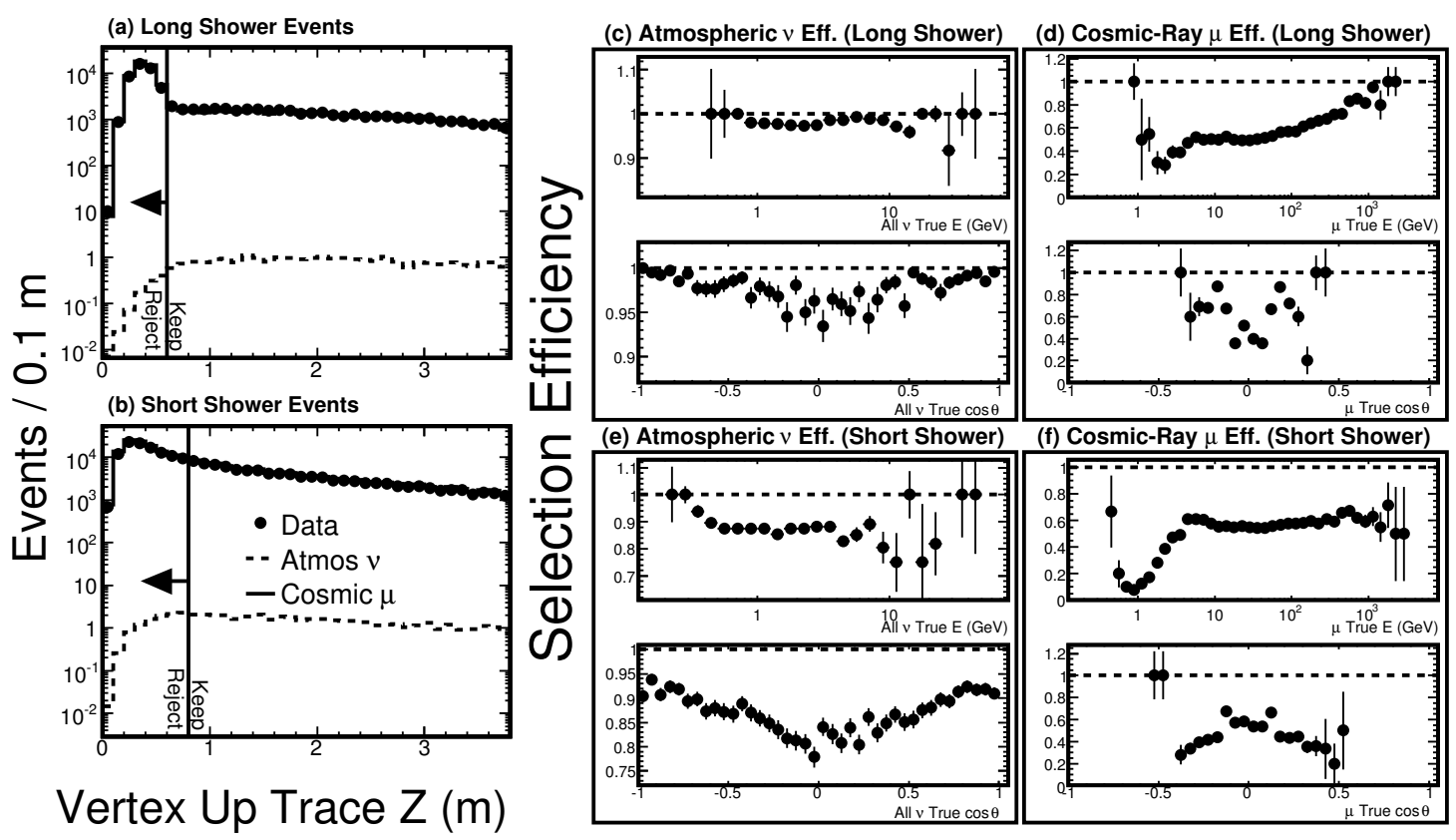

Figure 7.10: Shower Vertex Trace Z for the upward direction parity.

The directional resolution for showers is usually close but can often get the parity of the direction wrong. For this reason, the Trace $Z U p$ is devised for selection purposes. The cosmic-ray muon background consists entirely of muons that enter from above the detector. Of the two shower trace values (TraceZForward and TraceZReverse), the trace value that is derived from a direction pointing upward offers the best representation of the trace for the actual cosmic-ray muon. The TraceZUp is whichever of the two trace values was derived with $\cos \theta_{Y}>0$. Long shower events are required to have Trace $Z U p>0.6 m$ (Figure 17.10,a), and the short showers are required to have 
Trace $Z U p>0.8 m$ (Figure $7.10 \mathrm{~b}$ ).

\subsubsection{Shower Topology}

\section{- Eigenvalue Solutions for Moment of Inertia Tensor -}

When working in rigid body mechanics, it is convenient to parameterize the moments of the rigid body about all axes with the moment of inertia tensor. This tensor (I) satisfies the relationship between the vectorial angular momentum $(\vec{L})$ and angular velocity $(\vec{\omega})$ such that $\vec{L}=\mathbf{I} \cdot \vec{\omega}$. If a rigid body is a collection of points of mass $\left(m_{i}\right)$ located at $\vec{r}_{i}$ (where the zero point is defined as the rigid body's centroid), then the moment of inertia tensor is satisfied by the sum in dyadic notation of $\sum_{i} m_{i}\left(r_{i}{ }^{2} \mathbf{1}-\vec{r}_{i} \vec{r}_{i}\right)$ [100].

The moment of inertia tensor can be generalized from a rigid body to a showering event in the far detector by using strips as points with the strip energy in place of the point mass. In order to make the best comparison of event shapes between the simulated and observed sets, only strips with at least 2 pe are used. A showering event restricted into either $\mathrm{U}$ or $\mathrm{V}$-views produces two independent 2-D bodies. A single 3 -D body is made when views are combined, and each strip is assigned a longitudinal position based on the location of hits in adjacent planes. The 3-D body consists of a number of points in $(U, V, Z)$ each with an energy $E$. The energy weighted centroid of this body $\left(C_{U}, C_{V}, C_{Z}\right)$ is found by $C_{U}=\left[\sum E_{i} \times U_{i}\right] /\left[\sum E_{i}\right]$, etc. After all three coordinates have been shifted to the centroid frame $\left(U \rightarrow U-C_{U}\right.$, etc.), the dyadic form of moment of inertia tensor is expressed fully in Equation [7.1] All elements of the tensor are divided by the sum of the energy to remove the dependence on energy of the whole event.

$$
I=\frac{1}{\sum E_{i}}\left(\begin{array}{ccc}
\sum E_{i}\left(V_{i}^{2}+Z_{i}^{2}\right) & -\sum E_{i} U_{i} V_{i} & -\sum E_{i} U_{i} Z_{i} \\
-\sum E_{i} U_{i} V_{i} & \sum E_{i}\left(U_{i}^{2}+Z_{i}^{2}\right) & -\sum E_{i} V_{i} Z_{i} \\
-\sum E_{i} U_{i} Z_{i} & -\sum E_{i} V_{i} Z_{i} & \sum E_{i}\left(U_{i}^{2}+V_{i}^{2}\right)
\end{array}\right)
$$

The moment of inertia tensor is symmetric and real, which means that there must exist a unique coordinate axis which, upon transforming into the moment of inertia tensor, would diagonalize. The three axes of this transformation are called the "principal axes", and are found by solving the secular equation for the moment of inertia tensor 
$((I-\mathbf{1} \lambda) \cdot \vec{R}=0)$. The solution to the secular equation will contain three eigenvalues $\left(\lambda_{i}\right)$, each with a corresponding eigenvector $\left(\vec{R}_{i}\right)$. The three eigenvectors form the orthogonal $3 \mathrm{D}$ basis $\left(R_{0} \rightarrow X^{\prime}, R_{1} \rightarrow Y^{\prime}\right.$, and $\left.R_{2} \rightarrow Z^{\prime}\right)$ of the principal axes. If the moment of inertia tensor were constructed in this new $3 \mathrm{D}$ basis, it would have the form shown in Equation 7.2

$$
I=\frac{1}{\sum E_{i}}\left(\begin{array}{ccc}
\sum E_{i}\left(Y_{i}^{\prime 2}+Z_{i}^{\prime 2}\right) & 0 & 0 \\
0 & \sum E_{i}\left(X_{i}^{\prime 2}+Z_{i}^{\prime 2}\right) & 0 \\
0 & 0 & \sum E_{i}\left(X_{i}^{\prime 2}+Y_{i}^{\prime 2}\right)
\end{array}\right)
$$

The eigenvalues in the new 3D basis are three diagonal terms, which each translate into the scalar "energy-averaged" moment of inertia about their corresponding principal axis. The eigenvalues are referred to as "energy-averaged", as opposed to "energyweighted", because each term in the sum is weighted by the hit energy, and then each sum is divided by the total energy.

The size of the largest eigenvalue solution (MaxEVal) from the moment of inertia tensor is useful in distinguishing neutrino interactions from cosmic-ray muons. This will correspond to the largest scalar energy-averaged moment of inertia in the diagonalized basis. The MaxEVal would be larger for an event with a long straight energy deposition, and smaller for an event with diffuse hits and energy deposition that is stronger in a few planes.

Figure [7.11 a shows the distributions of MaxEVal for events with a shower that have more than 8 planes. Figure 7.11 b shows the distributions of MaxEVal for events with a shower that have 8 or less planes. The requirements are made that $\log _{10}$ MaxEVal $<-0.7$ for long shower events, and $\log _{10}$ MaxEVal $<-1.3$ for short shower events. The selection based on MaxEVal disfavors showering events with high energy. The efficiencies of selecting atmospheric neutrino interactions for long shower events (Figure 7.11.c) and short shower events (Figure 17.11.e) both have a point where the efficiency drops suddenly. This drop point is located at $E_{\nu} \sim 10 \mathrm{GeV}$ for the long shower events, and $E_{\nu} \sim 8 \mathrm{GeV}$ for short shower events. The difference between the break points is most likely due to a direct correlation between the MaxEVal and $E_{\nu}$. This correlation is not explored further in this thesis, but presents an interesting topic for future atmospheric neutrino analyses. 

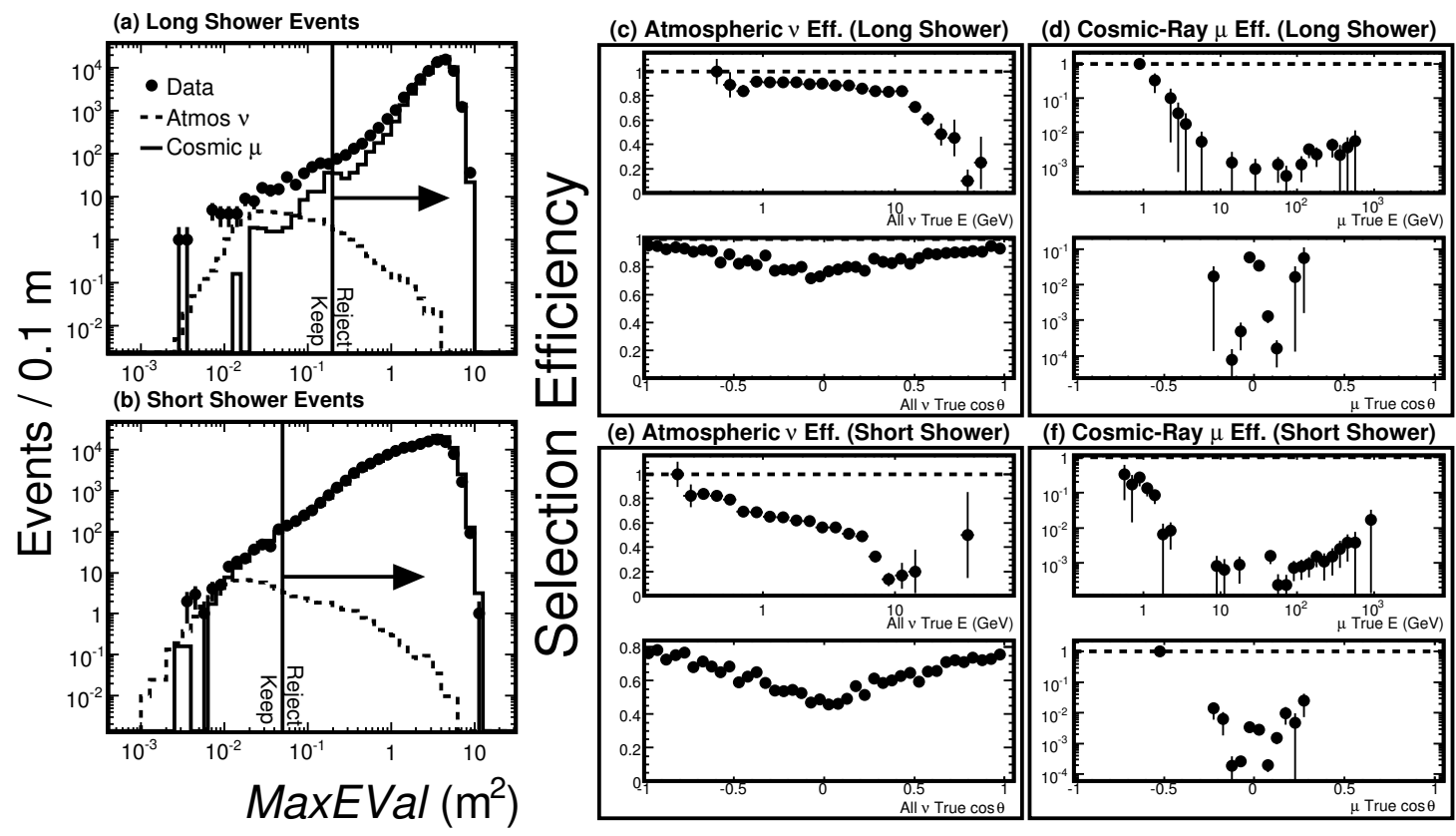

Figure 7.11: Maximum eigenvalue solution of the moment of inertia tensor

\section{- RMS Strips and Energy per Plane -}

The process by which a high-energy electron from a $\nu_{e} \mathrm{CC}$ interaction produces a showering event when passing through a thick absorber has been well described [101. As an electron passes by a nucleus, a photon is emitted due to Bremsstrahlung radiation [19]. The emitted photon will either scatter until it is photo-electrically absorbed or, if the photon has high enough energy, it may pair produce into an electron and positron. The pair-produced positron will annihilate with an electron in the absorber and produce two photons that will scatter until they are absorbed. The pair-produced electron will Bremsstrahlung radiating another photon, and the process starts over again. This iterative process will occur until the particles have lost all energy to the absorbing material. The result is an Electromagnetic Cascade [19, which will deposit energy along the axis of the initiating particle with the average energy deposition profile described by Equation 7.3 [19].

$$
\frac{d E}{d t}=E_{0} b \frac{(b t)^{a-1} e^{-b t}}{\Gamma(a)}
$$


$E_{0}$ is the initial electron energy and $t$ is the distance along the profile in terms of the radiation length $\left(t=X / X_{0}\right)$. The radiation length for iron is $X_{0}=1.76 \mathrm{~cm}$. If the shower is perpendicular to the plane face, then $t$ can be expressed in terms of the steel plane thickness $\left(W_{P}\right)$, and the number planes into the shower $(P)$. The energy profile by plane expressed in Equation 7.4 uses the new constant $C_{P}$ defined in Equation [7.5.

$$
\begin{aligned}
\frac{d E}{d P} & =E_{0} C_{P} \frac{\left(C_{P} P\right)^{a-1} e^{-C_{P} P}}{\Gamma(a)} \\
C_{P} & =b * \frac{X_{P}}{X_{0}}
\end{aligned}
$$

An electromagnetic shower that is initiated perpendicular to the plane face is expected to develop and deposit energy according Equation [7.4 The energy deposited in each steel plane is measured by energy deposited in the scintillator plane attached to that steel plane. If a shower is initiated other than perpendicular to the plane face, the sampling of the energy deposition profile should follow the shower direction. To evaluate the shower profile, the variance of the energy deposited in per plane is calculated. An electromagnetic shower deposits energy with a profile that peaks early in the shower and falls off quickly. Cosmic-ray muons that appear as a contained shower will often deposit much energy in a few planes, and little energy in the surrounding planes. The RMS about the mean of the strips hit per planes $\left(\left\langle(\text { strips } / \text { plane })^{2}\right\rangle^{1 / 2}\right)$ is used to identify neutrino candidates.

The energy deposition per plane is evaluated in two ways: first, based on the number of strips that are active in the plane, and second, based on the sum of charge in the plane. The RMS in the number of strips per plane (RMSStpPln), is shown in Figure 7.12. Events with a shower of 8 planes or less are required to have RMSStpPln $<2.0$ (Figure $7.12 \mathrm{a}$ ), while events with a shower of more than 8 planes are required to have RMSStpPln $<2.5$ (Figure $7.12 \mathrm{~b}$ ). The RMSEPln variable is profiles the energy deposited in each strip as measured by the pe count measured by the PMT. Events with a shower of 8 or less planes are required to have RMSEPln $<10^{2.1}$ (Figure [7.12] a), while events with a shower of more than 8 planes are required to have $R M S E P l n<10^{2.2}$ (Figure 7.13, b) 

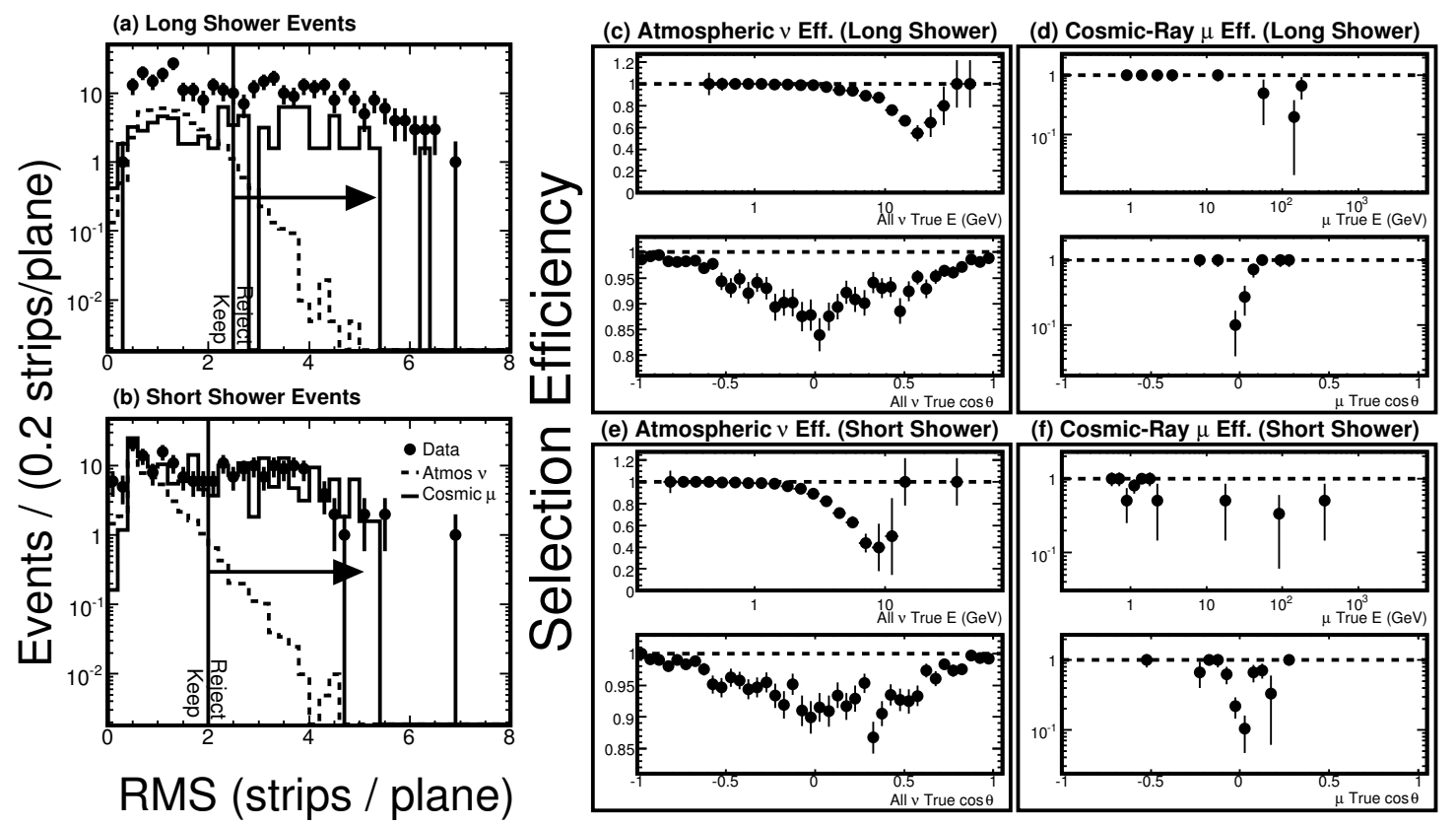

Figure 7.12: RMS of the number of strips hit per plane for showering events.
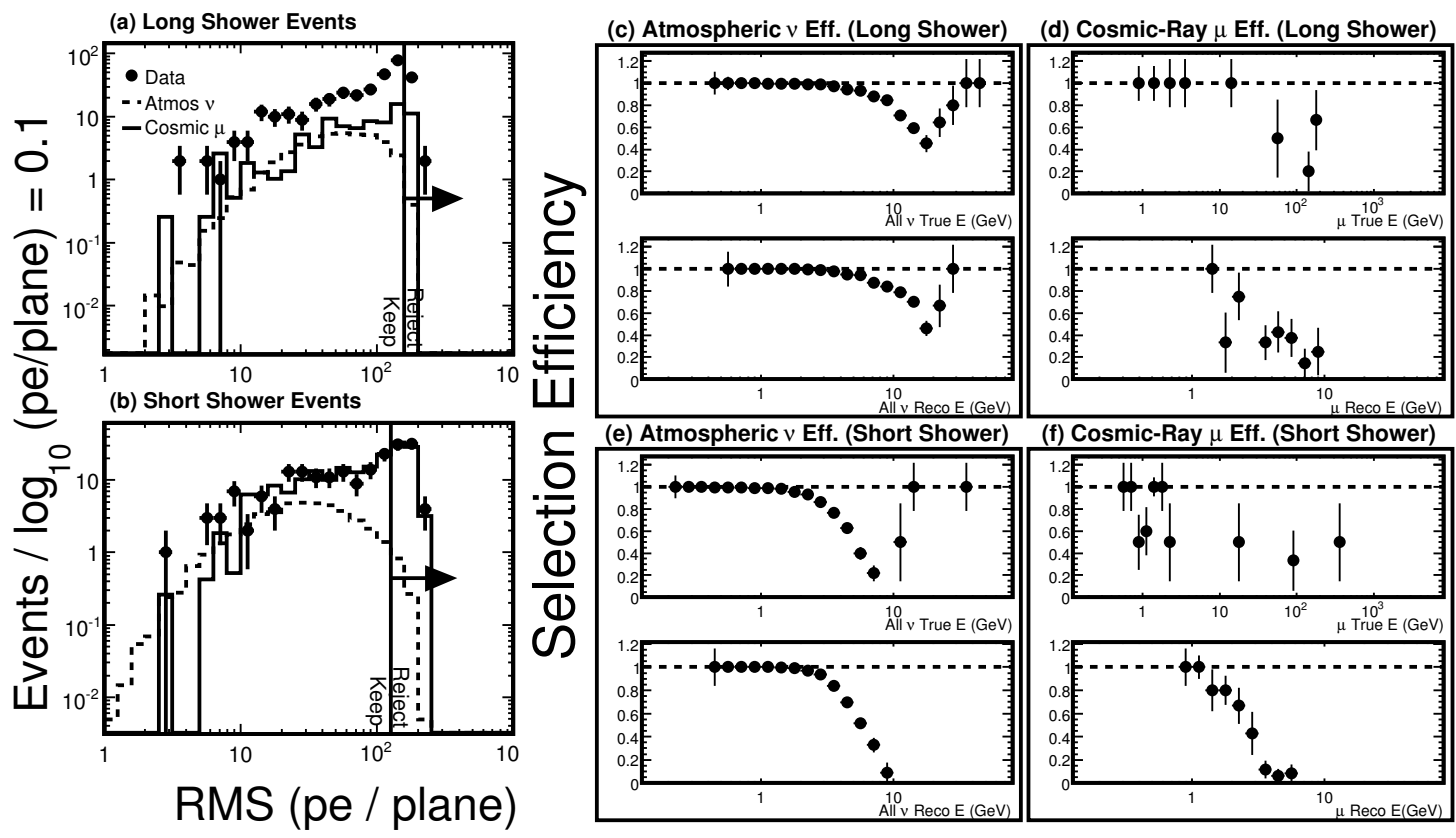

Figure 7.13: RMS of Energy deposited per plane in showering events. 


\subsubsection{Shower Selection Results}

Before applying the selections based on the trace and topology of the showering events, the events were divided into two classes: those with showers of $\leq 8$ planes, and those with showers of $>8$ planes. In both classes, event selection was applied based on values of the VtxUpTraceZ, MaxEVal, RMSStpPln, and RMSEPln. These four selections have been optimized based on a figure of merit (FOM) for the neutrino interaction signal to the cosmic-ray muon background. The FOM for a number of signal events $(S)$ and a number of background events $(B)$ is express in Equation [7.6.

$$
F O M(S, B)=\frac{S^{2}}{S+B}
$$

A sample events consists of $S+B$ events, and Poisson statistic predict that the number sample size will fluctuate inside of $(S+B) \pm \sqrt{S+B} 68 \%$ of the time that the count is made. The FOM represents square ratio of size of the signal $\left(S^{2}\right)$ to the size of the $68 \%$ fluctuations about the mean $(S+B)$. The FOM offers a quantity that represents the ability to see a number of signal events inside of a sample of signal and background.

The optimal values of the four selections that have been separated into long and short shower events (VtxUpTraceZ, MaxEVal, RMSStpPln, and RMSEPln) were not chosen progressively. Instead, all four selections were optimized simultaneously. An iterative procedure was used where all four selection values were brought close to the point where the FOM would be maximized, and FOM maximization points were recalculated with the newly selected set. This procedure was iterated until the FOM was maximized for all four of the selection values. A final optimization was performed by releasing each of the four selection individually, and placing the selection value at that point where the FOM was maximized. The resulting selection values are shown in Table 7.4

\begin{tabular}{|c|c|c|c|c|}
\hline & VtxUpTraceZ & MaxEVal & RMSStpPln & RMSEPln \\
\hline Shower $\leq$ Planes & $0.8 \mathrm{~m}$ & $10^{-1.4}$ & 2.0 strips $/$ plane & $10^{2.1} \mathrm{pe} /$ plane \\
Shower > 8 Planes & $0.6 \mathrm{~m}$ & $10^{-0.7}$ & 2.5 strips $/$ plane & $10^{2.2} \mathrm{pe} / \mathrm{plane}$ \\
\hline
\end{tabular}

Table 7.4: Optimized selection values of shower trace and topology variable, with optimization made by maximizing the FOM 
The event numbers resulting from the shower selection process are shown in Table 7.4.5. The signal (neutrino interactions) to background (cosmic-ray muons) ratio for the short shower set is 1:2100 after the shower quality selections, and 1:1.3 after the shower trace and topology selections. For the long shower set, the signal to background ratio of 1:3200 after the shower quality selections is increased to 1.6:1 after the shower trace and topology selections.

\begin{tabular}{|c|c|c|c|c|c|c|}
\hline & \multicolumn{7}{|c|}{ Monte Carlo } \\
& Data & $\stackrel{(-)}{\nu}_{e} \mathrm{CC}$ & $\stackrel{(-)}{\nu}_{\mu} \mathrm{CC}$ & $\nu$ NC & $\mathrm{CR} \mu$ & $\mathrm{CR} \mathrm{N}$ \\
\hline Event Quality & 512482 & 69.2 & 42.0 & 22.9 & 550653 & 40.6 \\
+ Shower Quality & 345955 & 68.4 & 41.1 & 22.6 & 266983 & 29.4 \\
\hline \hline Shower Planes $\leq 8$ & 240938 & 41.9 & 27.3 & 10.6 & 258477 & 19.6 \\
\hline + Vertex Up Trace Z & 135109 & 38.5 & 21.7 & 9.36 & 135109 & 12.6 \\
+ Maximum MOI Eigenvalue & 200 & 28.9 & 10.0 & 5.87 & 150.7 & 1.62 \\
+ RMS Strip, PE per Plane & 91 & 27.0 & 9.78 & 5.65 & 49.2 & 1.62 \\
\hline \hline Shower Planes > 8 & 512482 & 69.2 & 42.0 & 22.9 & 550653 & 40.6 \\
\hline + Vertex Up Trace Z & 61801 & 25.9 & 13.0 & 11.3 & 60065 & 6.64 \\
+ Maximum MOI Eigenvalue & 332 & 24.4 & 9.78 & 9.85 & 73.0 & 0.97 \\
+ RMS Strip, PE per Plane & 153 & 23.1 & 9.24 & 9.34 & 25.4 & 0.81 \\
\hline \hline Full Showering Sample & 244 & 50.1 & 19.0 & 15.0 & 74.5 & 2.43 \\
\hline
\end{tabular}

Table 7.5: Number of events for topology selections on showering events with shower of $>8$ planes.

The showering event selection results in table 7.4.5 are displayed progressively, but the values of the four selection criteria in this table were optimized simultaneously so this is deceptive. One method to conceptualize the effect of simultaneous selections is to construct a $4 \times 4$ "selection coincidence matrix" $(\mathbb{M})$ that demonstrates how each selection criterion coincides with the others. The showering data event selection coincidence matrices are shown in Table 17.6, while the same matrices for atmospheric $\nu_{e}$ CC interactions are shown in Table 7.7. Each matrix row and column represents one selection, and each matrix element contains the number of events that are removed by both the row selection criteria and the column selection criteria. The $\mathrm{i}^{\text {th }}$ diagonal element in the matrix $\left(\mathbb{M}_{i i}\right)$ contained the number of events that were discarded by the $i^{\text {th }}$ selection criteria.

In addition to the $4 \times 4$ matrix, a 4 vector $(\mathbf{U})$ is constructed to show the unique 
quality of each selection criteria. The $\mathrm{i}^{\text {th }}$ element of this vector is the number of events that are uniquely discarded by the $\mathrm{i}^{\text {th }}$ selection criteria.

\begin{tabular}{|c|c|c|c|c|c|}
\hline $\mathbb{M}_{L S}$ (Data) & UpTraceZ & MaxEVal & RMSStpPln & $R M S Q P \ln$ & $\mathbf{U}_{L S}$ (Data) \\
\hline UpTraceZ & 42887 & 42846 & 2428 & 48 & 19 \\
\hline MaxEVal & 42846 & 104315 & 9043 & 180 & 54832 \\
\hline RMSStpPln & 2428 & 9043 & 9244 & 229 & 136 \\
\hline$R M S Q P \ln$ & 48 & 180 & 229 & 231 & 1 \\
\hline $\mathrm{M}_{S S}$ (Data) & & & & & $\mathbf{U}_{S S}$ (Data) \\
\hline UpTraceZ & 105819 & 105672 & 46309 & 9517 & 101 \\
\hline MaxEVal & 105672 & 240570 & 120400 & 24352 & 60319 \\
\hline RMSStpPln & 46309 & 120400 & 120531 & 23626 & 41 \\
\hline RMSQPln & 9517 & 24352 & 23626 & 24449 & 9 \\
\hline
\end{tabular}

Table 7.6: Selection coincidence matrices $\left(\mathbb{M}_{L S}\right.$ and $\left.\mathbb{M}_{S S}\right)$ and unique selection vectors $\left(\mathbf{U}_{L S}\right.$ and $\left.\mathbf{U}_{S S}\right)$ for showering data events for both long showers $(L S)$ and short showers $(S S)$.

\begin{tabular}{r|rrrr||l}
$\mathbb{M}_{L S}\left(\nu_{e} \mathrm{CC}\right)$ & UpTraceZ & MaxEVal & RMSStpPln & RMSQPln & $\mathbf{U}_{L S}\left(\nu_{e} \mathrm{CC}\right)$ \\
\hline UpTraceZ & 0.28 & 0.039 & 0.024 & 0.0049 & 0.22 \\
MaxEVal & 0.039 & 1.50 & 0.16 & 0.015 & 1.30 \\
RMSStpPln & 0.024 & 0.16 & 1.01 & 0.12 & 0.99 \\
RMSQPln & 0.0049 & 0.015 & 0.12 & 0.35 & 0.23 \\
\hline \hline $\mathbb{M}_{\left.S S \text { ( } \nu_{e} \text { CC }\right)}$ & \multicolumn{1}{|c}{} & & & $\mathbf{U}_{S S}\left(\nu_{e} \mathrm{CC}\right)$ \\
\hline UpTraceZ & 3.38 & 1.63 & 0.40 & 0.21 & 1.54 \\
MaxEVal & 1.63 & 11.16 & 1.51 & 0.61 & 8.10 \\
RMSStpPln & 0.40 & 1.51 & 3.16 & 0.96 & 1.03 \\
RMSQPln & 0.21 & 0.61 & 0.96 & 1.65 & 0.46
\end{tabular}

Table 7.7: Selection coincidence matrices $\left(\mathbb{M}_{L S}\right.$ and $\left.\mathbb{M}_{S S}\right)$ and unique selection vectors $\left(\mathbf{U}_{L S}\right.$ and $\left.\mathbf{U}_{S S}\right)$ for showering $\nu_{e} \mathrm{CC}$ events for both long showers $(L S)$ and short showers $(S S)$.

There are still two problems with the shower set that need to be addressed before analyzing neutrino candidates. The signal to noise ratio is still small and the background is not well understood. Both of these issues are addressed when the selection is enhanced further by using the cosmic-ray veto shield over the detector, which will be discussed in Section 7.6 . 


\subsection{Track Selection}

A track-like event is defined as an event that passes the data quality requirements and pre-selection (Section [6.4), has a single track with any number of accompanying showers (Section 17.1), and is defined as either fully or partially-contained by energy containment (Section 6.4.1). The set of track-like events has been divided into three containment types: fully-contained (FC), partially-contained downward (PCDN) and partially-contained upward (PCUP). The detector Y-coordinate is used to distinguish between upward and downward tracks. A track is defined as upward if its vertex is below its end $(V t x Y<E n d Y)$, and the reconstructed direction of the vertex is pointing up $\left(\cos \theta_{V t x} Y>0\right)$. A track is defined as downward if the vertex is above the end $(V t x Y>$ $E n d Y)$ and the reconstructed direction of the vertex is pointing down $\left(\cos \theta_{V t x}<<\right)$.

The numbers of events in Table 7.1 reflect that the neutrino-interaction signal to background ratios are 1:180 for FC, 1:510 for PCDN, and 1:80 PCUP. To increase the signal to background ratio, events that fail track and event quality criteria are removed from all three sets. The FC and PCDN sets are then isolated and reduced based on hit shape and charge deposition around the track vertex. The PCUP set is isolated and reduced based on timing parameters to remove downward cosmic-ray muons that mis-reconstructed as upward.

\subsubsection{Track and Event Quality}

\section{- Maximum Plane Charge -}

In the selection of showering events (Section [7.4.1), a selection was made based on the maximum charge deposited in a single plane. The same selection is made on track-like events, where there should be no more the 500 pe deposited in a single plane.

Figure 7.14 a shows the distribution of maximum charge in a single plane for tracklike events, and is analogous to Figure [7.8 a for showering events. The efficiency of this selection for both atmospheric neutrinos interactions (Figure 7.14,b) and cosmicray muons (Figure $7.14 \mathrm{c}$ ) is lowest for high-energy interactions, where the likelihood of depositing a large amount of energy in a single plane is the highest. The efficiency is also lower for cosmic-ray muons and atmospheric neutrino interactions where the incoming particle has a steep angle relative to the face of the plane $\left(\cos \theta_{Z} \sim 0\right)$. 
(a) Max. Single Plane Charge

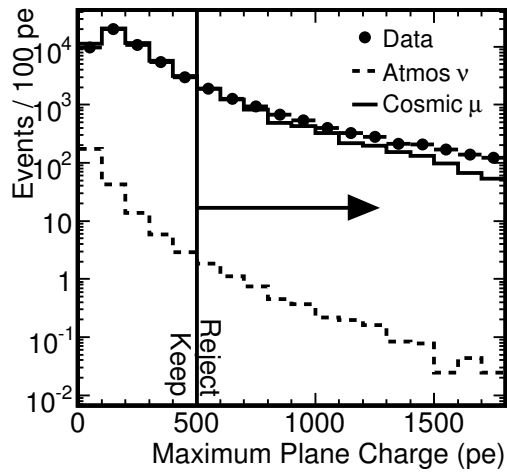

(b) Atmospheric $v$ Efficiency

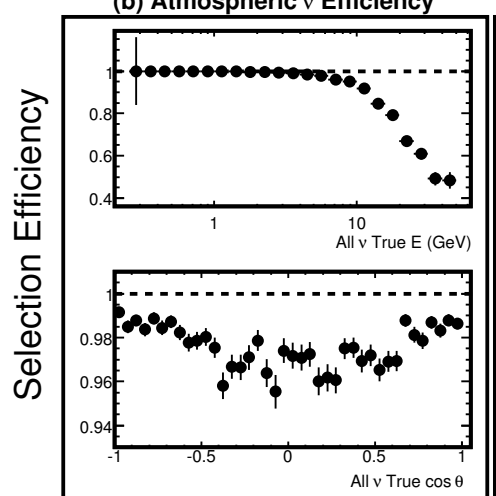

(c) Cosmic-Ray $\mu$ Efficiency

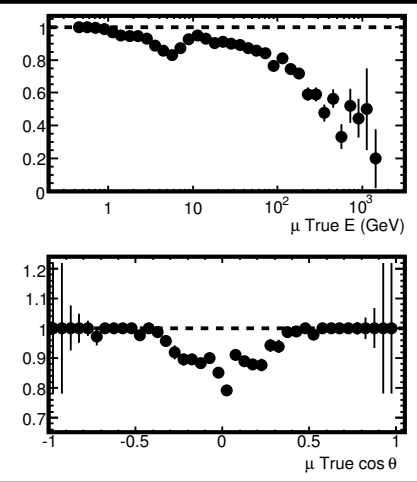

Figure 7.14: Maximum charge deposited in a single plane for track-like events.

\section{- Track Charge Completeness -}

In order to assure track-like events that are well reconstructed, the completeness of the track is measured in terms of hits in the event that are associated with the track. At least $40 \%$ of all charge in an event must occur within \pm 1 strip of the track. A selection like this, that is based on the showering aspect of an event, will remove $\nu_{\mu}$ CC events with significant showering aspect (i.e. a DIS neutrino interaction with an energetic hadronic shower).

(a) Track Hit Completeness

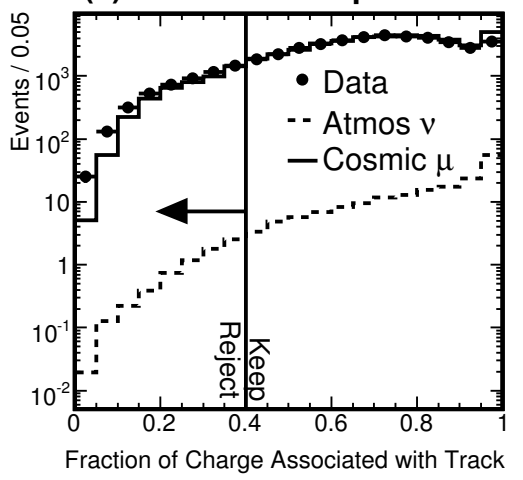

(b) Atmospheric $v$ Efficiency

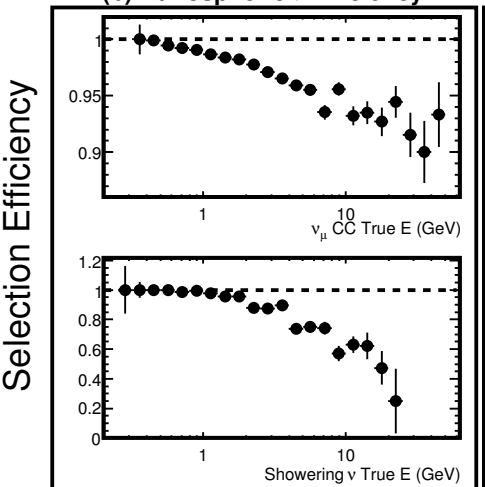

(c) Cosmic-Ray $\mu$ Efficiency

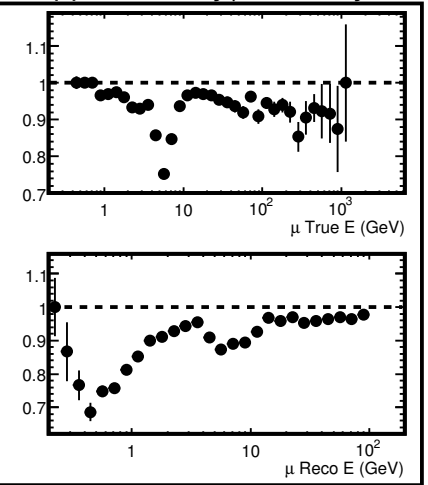

Figure 7.15: Fraction of charge associated with a track.

Figure [7.15] a shows the distribution of the fraction of the charge associated with the track (TrkChargeFrac), where the data has a surplus at the lower completeness over the cosmic-ray muon simulated set. This surplus is a result of data events having 
more low energy noise hits than the simulated events. The bins with the lowest value of TrkChargeFrac are filled by events that are considered reconstruction failures, where the track finder could not make a track out of most of the hits. The failure occurs more often in the data than expected, and it is considered beneficial to remove these events from the data set.

The efficiency of this selection on the atmospheric neutrino interactions (Figure 7.15.b) is lower for higher energies of both track-like and showering neutrino interactions. The showering neutrino interactions have a much lower efficiency than the track-like neutrino interactions at higher energies, and the overall efficiency is lower for the showering than track-like neutrino interactions.

The cosmic-ray muon selection efficiency in Figure 7.15.c is shown as a function of both true and reconstructed muon energy. The efficiency for the true energy is strongly peaked at $\mathrm{E}_{\mu} \sim 5 \mathrm{GeV}$. Simulated cosmic-ray muon events that are selected against in this range are largely composed of events where the muon enters the detector a steep backward angle $\left(\cos \theta_{Z} \stackrel{\sim}{<} 0\right)$, and is bent by the magnetic field until is it traveling forward in the detector. The hits that the muon makes before turning around are not registered as part of the track, and the result is that a significant fraction of the charge in the event is not associated with the track.

\section{- Track Like Planes and Track Length -}

The next step to select well-reconstructed track-like events is to set limits on the number of planes that the track crosses, and the track-like quality of those planes. A track-like plane is defined as a plane with $<80$ pe and $80 \%$ of strip charge within \pm 1 strip of the reconstructed track hits. Fully-contained track-like events must have at least 5 track-like planes, and partially-contained events must have at least 6 track-like planes, as shown in Figure 7.16]a.

An event that fails the track-like plane selection may do so either because there are too few overall active planes in the event, or because there are not enough active planes that qualify as track-like. Tracks are constructed with a minimum of 5 planes, so events that fail a five or six track-like plane selection will likely have sufficient overall active planes and insufficient track-like planes. The track-like selection efficiency for atmospheric neutrino interactions (Figure [7.16, b) is not strongly energy dependent. For 

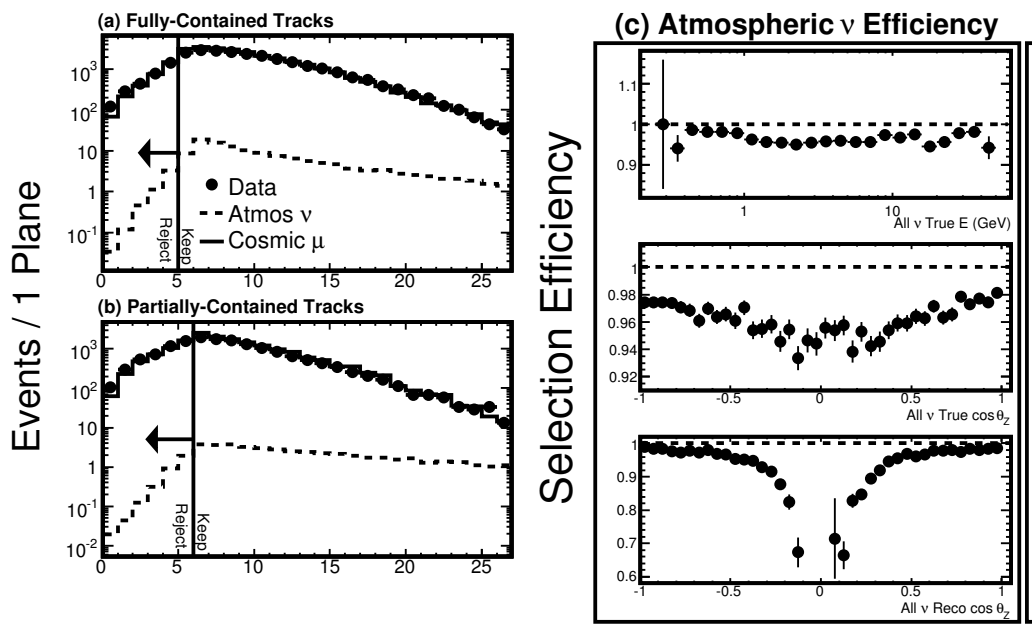

(d) Cosmic-Ray $\mu$ Efficiency

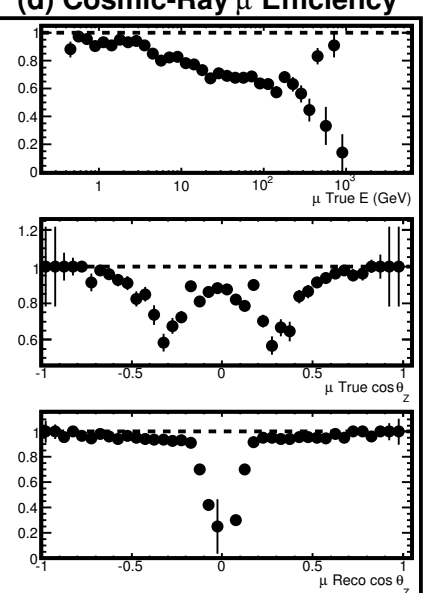

Figure 7.16: Count of track-like planes in fully and partially-contained tracks, requiring that FC events have at least 5 track-like planes and PC events have at least 6 track-like planes.

cosmic-ray muons (Figure [7.16]c), the efficiency is lower for higher energies.

The distribution of angles from the plane face $\left(\cos \theta_{Z}\right)$ demonstrates a dependence for both cosmic-ray muons and neutrino interactions that is stronger for the reconstructed angle than for the true angle. A particle that is removed by the track-like plane selection is steep enough relative to the plane face to hit several strips while passing through an active plane, but not so steep that it would be removed in the selection on the maximum charge in a single plane. This causes the double hump structure in the efficiency as a function of the true $\cos \theta_{Z}$ for cosmic-ray muons (Figure [7.16] b).

The "track length" expressed as track planes is defined as the number of planes that have a hit that is associated with the track. Fully-contained track-like events must have at least 8 track planes, and partially-contained track-like events must have at least 10 track planes; the extra planes in PC is to secure tracks that can be more easily identified for direction, as shown in Figure 7.17a.

The selection based on track planes disfavors low energy interactions, as shown in the selection efficiency for atmospheric neutrino interactions (Figure 7.17 b) and cosmicray muons (Figure $7.17 \mathrm{c}$ ). The selection efficiency is lower for atmospheric-neutrino interactions near $\cos \theta_{Z} \sim 0$, and lower for cosmic-ray muons for $\cos \theta_{Z}>0.3$. A cosmic-ray muon that is steep relative to the plane face will only appear contained and 


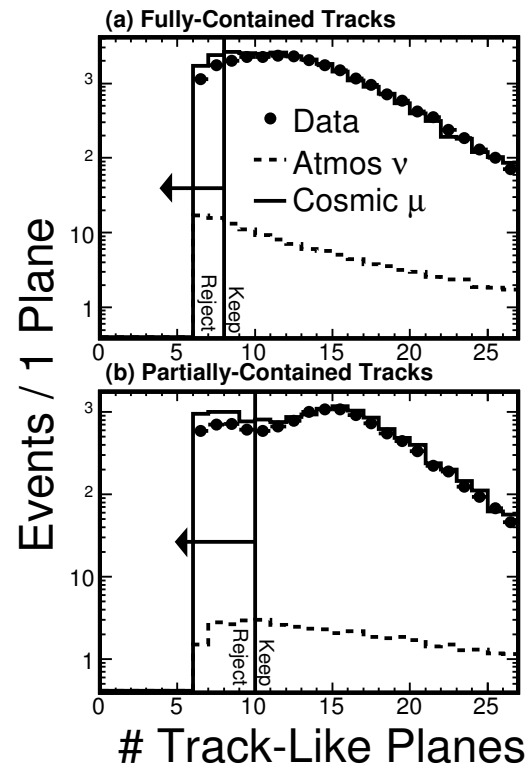

(c) Atmospheric $\vee$ Efficiency

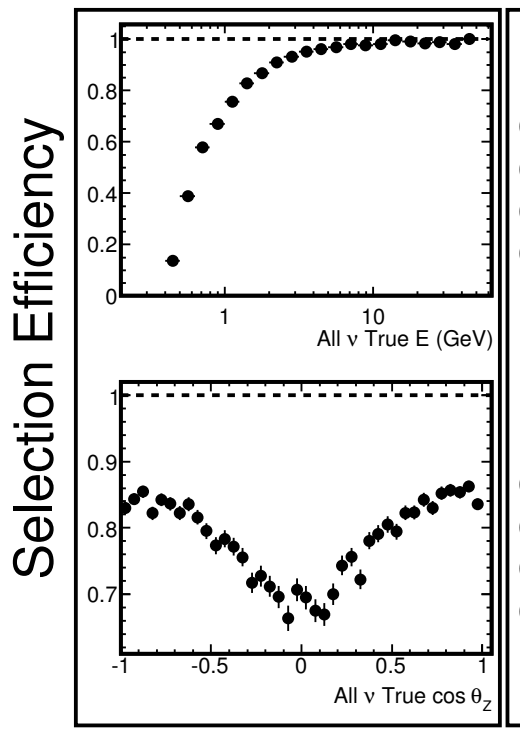

(d) Cosmic-Ray $\mu$ Efficiency

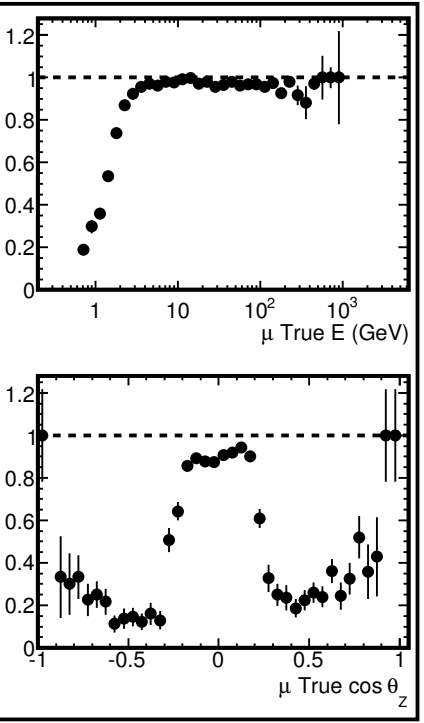

Figure 7.17: Count of track planes in fully and partially-contained tracks. Require that FC events have at least 8 track-like planes and PC events have at least 10 track-like planes.

produce a significant track if it is energetic enough to turn in the magnetic field, thus the background events removed by this selection are low energy muons that have a large angle relative to the plane face.

\section{- Selection Results -}

\begin{tabular}{|c|c|c|c|c|c|c|}
\hline & \multirow[b]{2}{*}{ Data } & \multicolumn{5}{|c|}{ Monte Carlo } \\
\hline & & $\stackrel{(-)}{\nu}_{\mu} \mathrm{CC}$ & $\stackrel{(-)}{\nu}_{e} \mathrm{CC}$ & $\nu \mathrm{NC}$ & $\mathrm{CR} \mu$ & CR N \\
\hline Pre-Selected FC Tracks & 33457 & 150.6 & 10.2 & 7.18 & 30045 & 1.94 \\
\hline +Maximum Plane Charge & 30321 & 148.8 & 9.46 & 6.82 & 27638 & 1.30 \\
\hline +Track Completeness & 27504 & 145.1 & 8.82 & 6.02 & 25290 & 1.13 \\
\hline +Track Plane Counts & 21555 & 115.4 & 3.74 & 3.13 & 20098 & 1.13 \\
\hline
\end{tabular}

Table 7.8: Number of fully-contained track-like events through track and event quality selections. 


\begin{tabular}{|c|c|c|c|c|c|c|}
\hline & \multirow[b]{2}{*}{ Data } & \multicolumn{5}{|c|}{ Monte Carlo } \\
\hline & & $\stackrel{(-)}{\nu}_{\mu} \mathrm{CC}$ & $\stackrel{(-)}{\nu}_{e} \mathrm{CC}$ & $\nu \mathrm{NC}$ & $\mathrm{CR} \mu$ & CR N \\
\hline Pre-Selected PC Tracks & 24214 & 73.6 & 1.22 & 1.00 & 21496 & 6.80 \\
\hline +Maximum Plane Charge & 18584 & 70.9 & 1.12 & 0.94 & 17555 & 5.02 \\
\hline +Track Completeness & 16110 & 69.3 & 0.99 & 0.77 & 15641 & 2.75 \\
\hline +Track Plane Counts & 8938 & 55.0 & 0.21 & 0.19 & 9422 & 1.30 \\
\hline PC Downward Tracks & 8503 & 27.0 & 0.12 & 0.078 & 9006 & 1.13 \\
\hline PC Upward Tracks & 453 & 27.9 & 0.093 & 0.11 & 416 & 0.16 \\
\hline
\end{tabular}

Table 7.9: Number of partially-contained track-like events through track and event quality selections.

\subsubsection{Fully Contained and Partially Contained Downward Tracks}

Fully-contained (FC) and partially-contained downward (PCDN) track-like events are combined for further event selection for two reasons: they both use the shield to enhance event select (Section [7.6), and they both have a similar background of steep cosmic-ray muons that enter through gaps and imitate track-like events with a contained vertex.

Cosmic-ray muons that fake containment have a tendency to either deposit a large amount of energy around the track vertex, or have hits distributed in a highly scattered manner around the track vertex. For the selections described in this section, the topology of hits around the track vertex are used to discriminate between neutrinos and cosmic ray muons. PCDN track-like events have a vertex that is well defined as the contained track end, but both ends of the track in FC track-like events are contained. For shorter tracks with poorly defined timing, it is not always easy to determine which end of the track is the true event vertex.

The particle that creates a track-like event will be relativistic, and can be considered to have the speed of light. In order to label one track side as the vertex and the other as the end, two linear fits are made of the track hit position versus time constrained to the speed of light. For each fit direction the RMS deviation of the hit timing from the line is determined, and the lower of the two decides which track side will be used as the vertex $\left(R M S_{v t x}\right)$ and which side will be used as the end $\left(R M S_{\text {end }}\right)$. When $R M S_{v t x}$ is less than $R M S_{\text {end }}$ by a marginal amount, there is a greater risk of placing the vertex at the wrong track side.

The previous $\nu_{\mu} \mathrm{CC}$ analysis [4] selected FC events based topological parameters 
for hits surrounding the higher in $\mathrm{Y}$ of the track vertex or end. This method has been improved upon in this thesis. For the FC track-like events, the side of the track about which topological parameters are selected upon is based on the $R M S$ of the two fits and the heights of the track vertex $\left(V t x_{Y}\right)$ and end $\left(E n d_{Y}\right)$. The decision is made based on the following criteria:

- If the track end is higher in Y than the track vertex (Figure [7.18,a), then the track end may be used.

- If the track has $V t x_{Y}<E n d_{Y}$, the $R M S_{v t x}$ is less than $R M S_{\text {end }}$ by at least 0.5 ns (Figure [7.18,b), and $R M S_{v t x}<1.0 n s$ (Figure [7.18 c), then the track vertex must be used.

- If the track has low timing resolution $\left(R M S_{\text {end }}-R M S_{v t x}<0.5 \mathrm{~ns}\right.$, see Figure $7.18 \mathrm{~d}$ ), and the track vertex and end are within $50 \mathrm{~cm}$ of each other in $\mathrm{Y}$ $\left(\left|V t x_{Y}-E n d_{Y}\right|<50 \mathrm{~cm}\right.$, see Figure [7.18] e), then both the track vertex and end are examined for topological selections.

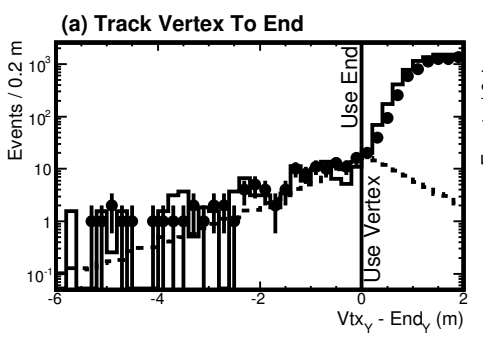

(d) Track Timing

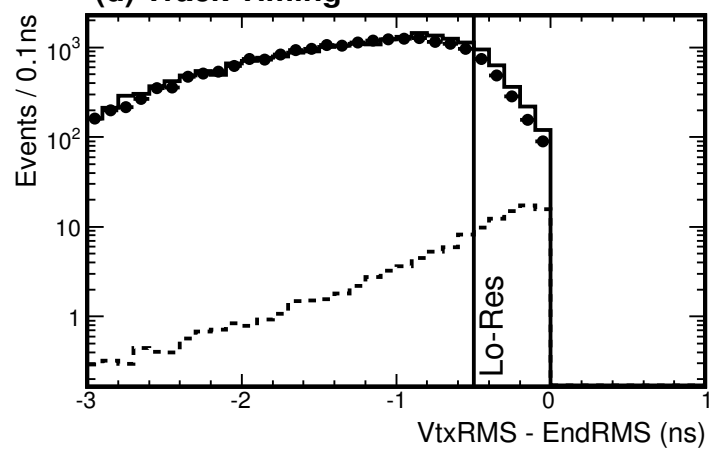

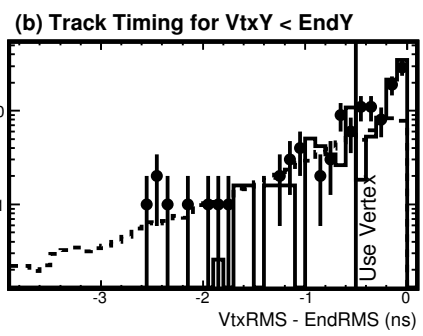

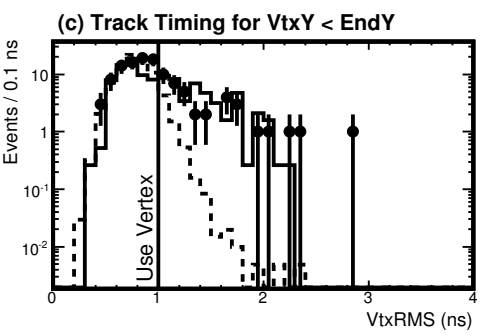

(e) VtxY - EndY For Lo-Res Events

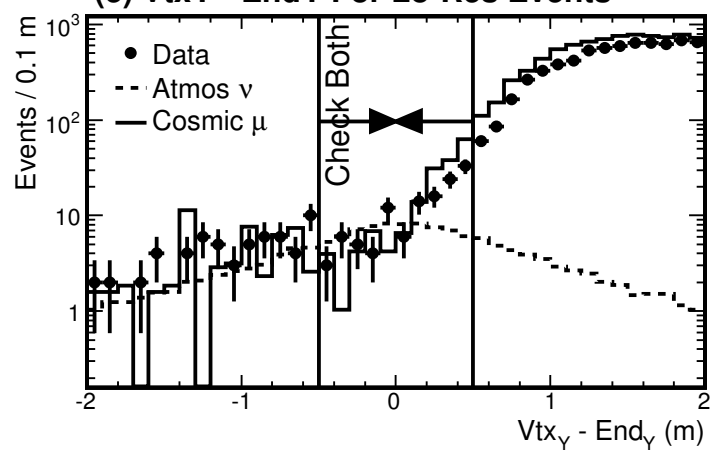

Figure 7.18: Fully-contained track vertex and end topology selection conditions. 
When the track vertex is referred to in this section, this will be the actual track vertex for PCDN track-like events. For FC track-like events, the track side that is chosen based on the criteria above.

\section{- Vertex Trace Z -}

The vertex trace was described for the shower selection in Section [7.4.3. The trace follows the vertex direction to the nearest edge of the detector to estimate where a particle might have entered to create the contained vertex. The Trace $Z$ projects this trace along the Z-axis, and thereby gives an estimate of the amount of steel the particle would have had to travel through undetected to create a contained vertex with the given direction.

(a) Best Track End Trace Z

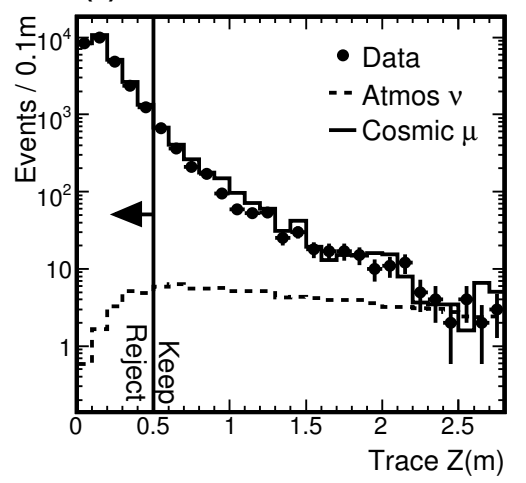

(b) Atmospheric $v$ Efficiency

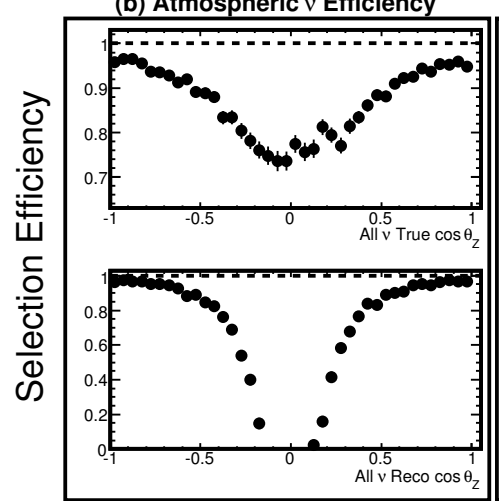

(c) Cosmic-Ray $\mu$ Efficiency

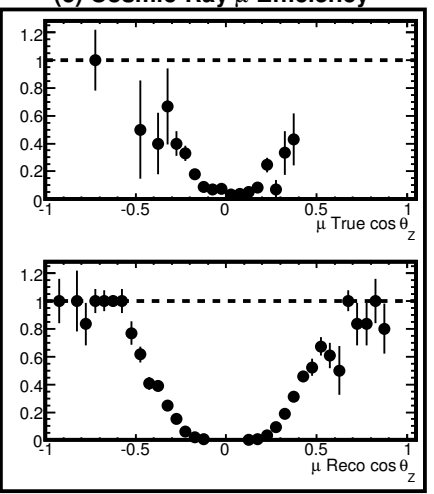

Figure 7.19: VtxTrace $Z$ for $\mathrm{FC}$ and $\mathrm{PCDN}$ track-like events, requiring that VtxTraceZ $>50 \mathrm{~cm}$.

The ambiguity in the shower direction forced the selection to be made based on the VtxTrace $Z$ solution that projects back along a direction for $\cos \theta_{Y}>0$, called $V t x U p \operatorname{Trace} Z$. A track has a well defined direction, so the VtxTrace $Z$ is used directly for track-like events. FC and PCDN events are required to have VtxTraceZ $<50$ cm. In Figure [7.19] a it is clear that this selection removes a significant fraction of the cosmic-ray muon background. The efficiency of the selection is lower for steeper angles relative to the plane face for both atmospheric neutrino interactions (Figure 7.19,b) and cosmic-ray muons (Figure $7.19 \mathrm{c}$ ). 


\section{- 10 Plane Track Vertex Window 2D Hit Distribution -}

When a cosmic-ray muon appears to be contained by turning around in the magnetic field, it may leave a scattered group of hits around the apparent event vertex. These hits are examined by looking at hits with $\geq 2$ pe in a 10 plane window centered around the track vertex. In each strip view, the mean and RMS charge weighted distance of the strip from the vertex is measured (MeanDelT and RMSDelT respectively). A large value of MeanDelT or RMSDelT would indicate that the event may have been initiated by a cosmic-ray muon.
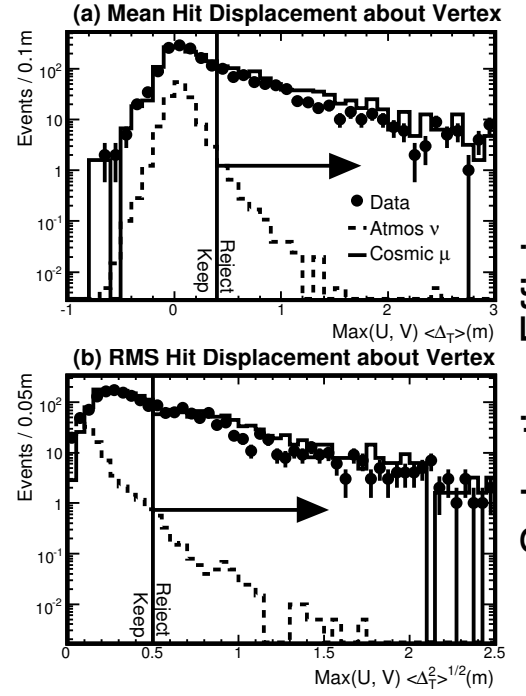

(c) Atmospheric $v$ Efficiency
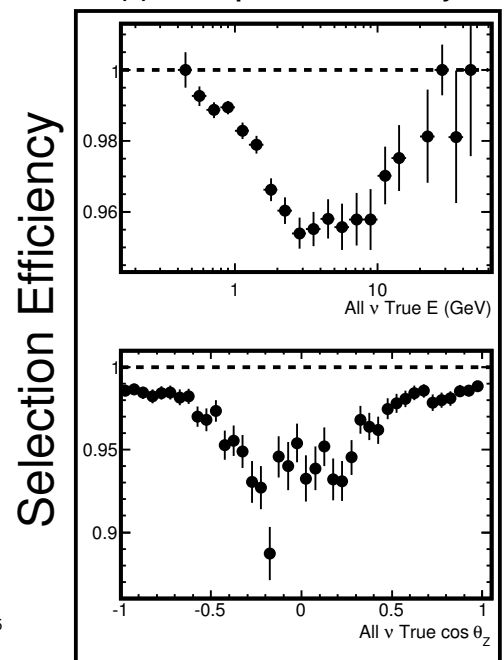

(d) Cosmic-Ray $\mu$ Efficiency

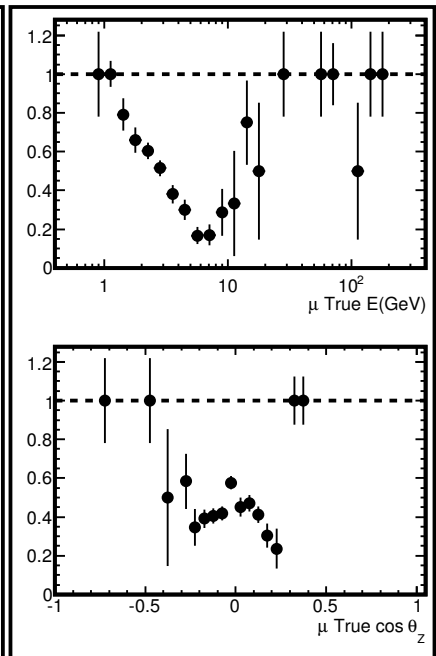

Figure 7.20: Maximum between $\mathrm{U}$ and $\mathrm{V}$ views of Mean and RMS displacement from track vertex for FC and PCDN track-like events.

Figure 7.20] a displays the distributions of MeanDelT, where the requirement is made that MeanDelT $>0.4 \mathrm{~m}$. Figure [7.20 b displays the distributions of RMSDelT, where the requirement is made that RMSDelT $>0.5 \mathrm{~m}$. These selections will tend to disfavor high energy interactions from both atmospheric neutrinos (Figure [7.20 c) and cosmic-ray muons (Figure $7.20 \mathrm{~d}$ ).

\section{- 10 Plane Track Vertex Window 3D Hit Displacement -}

A set of hits around the track vertex were used to examine the mean and RMS displacement in each view. These hits also have a $3 \mathrm{D}$ position that was determined when 
measuring the charge containment (Section 6.4.1). Events that cross 20 planes will have traversed $\sim 1.2 \mathrm{~m}$, and will consist of muons that are mostly $<10 \mathrm{GeV}$. For track-like events with a track that consists of 20 or fewer planes, the $3 \mathrm{D}$ distance from the $\geq 2$ pe hits around the vertex is required to be $<1.25 \mathrm{~m}$.

(a) Max 3D Hit Disp from Vtx

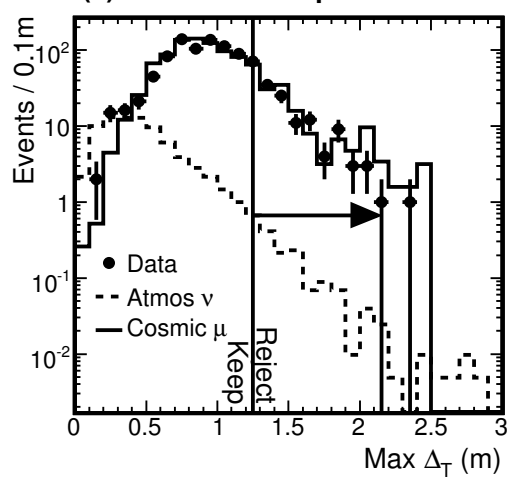

(b) Atmospheric $v$ Efficiency

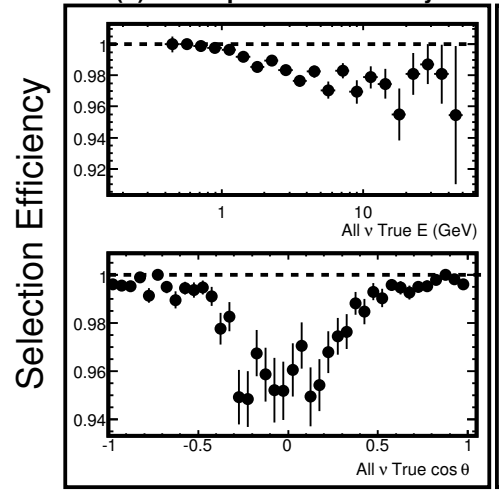

(c) Cosmic-Ray $\mu$ Efficiency

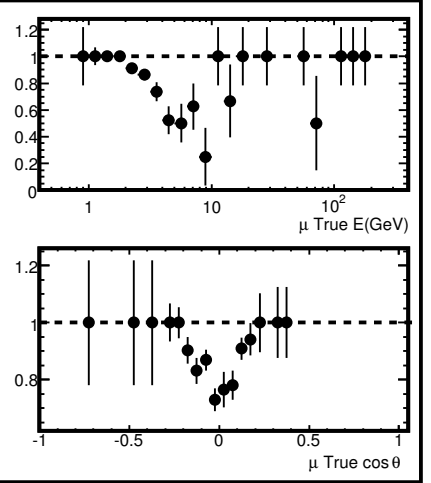

Figure 7.21: Maximum 3D displacement around the track vertex of all hits with $\geq 2$ pe for FC and PCDN track-like events.

The distribution of the maximum 3D displacement (VtxRMax) for short tracks is shown in Figure 7.21 a. The efficiencies in Figures 7.21, b and 7.21 c (atmospheric neutrino interactions and cosmic-ray muons respectively) demonstrate a consistently lower efficiency for both higher energy interactions, and those that have a smaller $\cos \theta_{Z}$.

\section{- 10 Plane Track Vertex Window Charge Deposition -}

The hits in the 10 plane window around the track vertex have been examined topologically, and are now examined for cumulative charge deposition. To distinguish cosmic-ray muons from atmospheric neutrino interactions, the quantity examined is the maximum charge deposited in one of the 10 planes around the vertex. The selection made earlier based on the MaxPlaneCharge required that no single plane has more than 500 pe, and this selection is extended to the 10 planes around the vertex (MaxTrkVtxPlaneCharge).

The selection based on the MaxTrkVtxPlaneCharge is targeted at short track events (TrkPlanes $\leq 20)$. Tracks that are either nearly parallel to the plane face or at a steep zenith angle are selected based on the MaxTrkVtxPlaneCharge. In Figures 

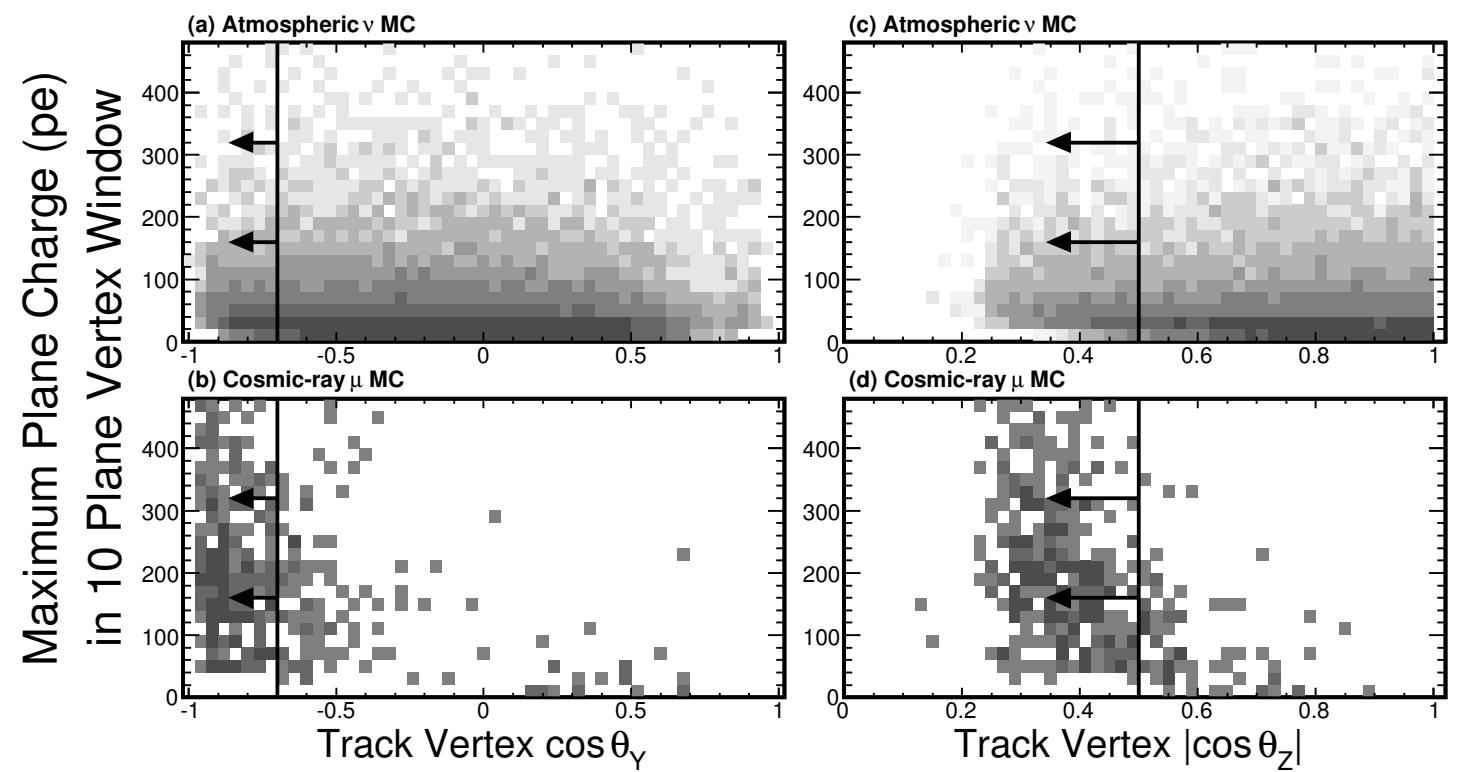

Figure 7.22: Maximum charge deposited in a single plane in a 10 plane track vertex window versus the vertex angle.

7.22 $\mathrm{a}$ and 7.22. b, the track vertex zenith angle is plotted against MaxTrkVtxPlaneCharge for atmospheric neutrino interactions and cosmic-ray muons respectively. Similarly, Figures $7.22 \mathrm{c}$ and 7.22] d contain the track vertex angle relative to the plane face plotted against MaxTrkVtxPlaneCharge for atmospheric neutrino interactions and cosmic-ray muons respectively.

Based on the distributions in Figure 7.22 only events that satisfy the vertex direction requirement $\cos \theta_{Y}<-0.7$ or $\left|\cos \theta_{Z}\right|<0.5$ have the MaxTrkVtxPlaneCharge examined for further selection. Figure 7.23 a shows the MaxTrkVtxPlaneCharge distributions for short tracks with the appropriate vertex direction, which is required to be no greater than 100 pe. This efficiency of this selection falls near the appropriate values of $\cos \theta_{Z}$ and $\cos \theta_{Y}$ for atmospheric neutrino interactions (Figure $7.23 \mathrm{~b}$ ) and cosmic-ray muons (Figure $7.23 \mathrm{c}$ ).

\section{- Selection Results -}

Table 7.10 contains the number of events remaining after each of the selections described in this section are applied. From the simulated sets, the atmospheric neutrino selection 
(a) 10 Plane Vertex Window for

(b) Atmospheric $v$ Efficiency

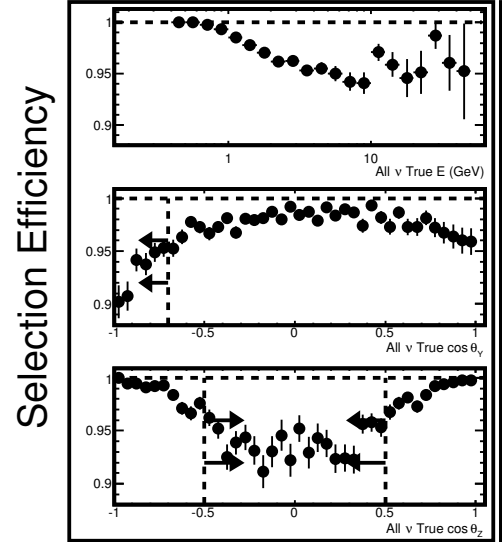

(c) Cosmic-Ray $\mu$ Efficiency

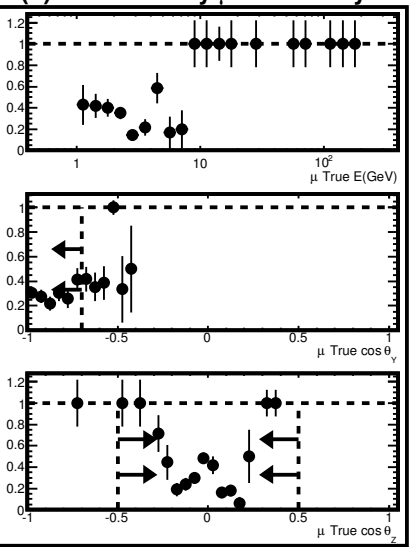

Figure 7.23: Maximum single plane charge in a 10 plane track vertex window, for $\left.\cos \theta_{Y}<-0.7\right)$ or $\left|\cos \theta_{Z}\right|<0.5$.

\begin{tabular}{|c|c|c|c|c|c|c|}
\hline & \multirow[b]{2}{*}{ Data } & \multicolumn{5}{|c|}{ Monte Carlo } \\
\hline & & $\stackrel{(-)}{\nu}_{e} \mathrm{CC}$ & $\stackrel{(-)}{\nu}_{\mu} \mathrm{CC}$ & $\nu \mathrm{NC}$ & $\mathrm{CR} \mu$ & CR N \\
\hline Quality FC Tracks & 21555 & 115.4 & 3.74 & 3.13 & 20098 & 1.13 \\
\hline+ VtxTraceZ & 1525 & 102.2 & 3.42 & 2.92 & 1404 & 0 \\
\hline + Vertex Hits Topology & 762 & 98.4 & 3.26 & 2.71 & 609. & 0 \\
\hline + Vertex Charge & 229 & 96.2 & 3.2 & 2.6 & 171. & 0 \\
\hline Q Quality PCDN Tracks & 8503 & 27.0 & 0.12 & 0.078 & 9006 & $\overline{1.13}$ \\
\hline$+V t x \operatorname{Trace} Z$ & 336 & 25.0 & 0.11 & 0.078 & 454 & 0 \\
\hline + Vertex Hits Topology & 85 & 23.9 & 0.10 & 0.073 & 76.1 & 0 \\
\hline+ Vertex Charge & 50 & 22.7 & 0.093 & 0.059 & 44.4 & 0 \\
\hline
\end{tabular}

Table 7.10: Number of Fully-contained and partially-contained downward going tracklike events through track topology selection. 
signal to background ratio is measured to be 1:1.7 and 1:1.9 for the FC and PCDN sets respectively. The size of the simulated cosmic-ray muon set has a large uncertainty at this point due to the large scale factor used to match this set to the data exposure. To rectify this, the veto shield is used to both further reduce the cosmic-ray muon background, and to arrive at a better measurement of the contribution of this background to signal events. This will be discussed in Section [7.6, following the discussion of the selection of neutrino interactions in the PCUP track-like event set.

\subsubsection{Partially-Contained Upward Tracks}

The primary background to the partially contained upward tracks is from downward going muons that have been mis-reconstructed to point upward. To isolate these, the directional timing resolution is used to achieve background separation.

\section{- Upward Direction Selection -}

All tracks are constructed with a vertex and an end. In order to determine which side of a track is the vertex, the track is fit for both possible directions assuming that the particle has the speed of light. For each of these fits, the RMS deviation of the observed track hit time from the expected hit time is measured. The lesser RMS of the two fits $\left(R M S_{v t x}\right)$ determines the vertex, and the greater RMS $\left(R M S_{\text {end }}\right)$ determines the end. Figure 7.24 shows how direction is determined for a forward track and a reverse track. In both cases, the solid line shows the winning direction.

For the upward events examined in this portion of the event selection, the RMS deviations from the vertex and end fits are then redefined to $R M S_{v t x} \rightarrow R M S_{u p}$ and $R M S_{\text {end }} \rightarrow R M S_{\text {down }}$. By definition $R M S_{u p}<R M S_{d o w n}$, and in Figure [7.25]a, the difference between $R M S_{u p}$ and $R M S_{\text {down }}$ is shown. It is required that $R M S_{u p}-$ $R M S_{\text {down }}<-0.5$ to select tracks that have a high probability that they are upward going.

The atmospheric neutrino interactions removed by this selection are primarily from low energy neutrinos, as seen in Figure 7.25]b. Figure 7.25.c demonstrates that the same is true of the cosmic-ray muons selected to be discarded. This selection is effective at removing a large portion of the cosmic-ray muon background, but there are still background events that need to selected against more aggressively than has been done 


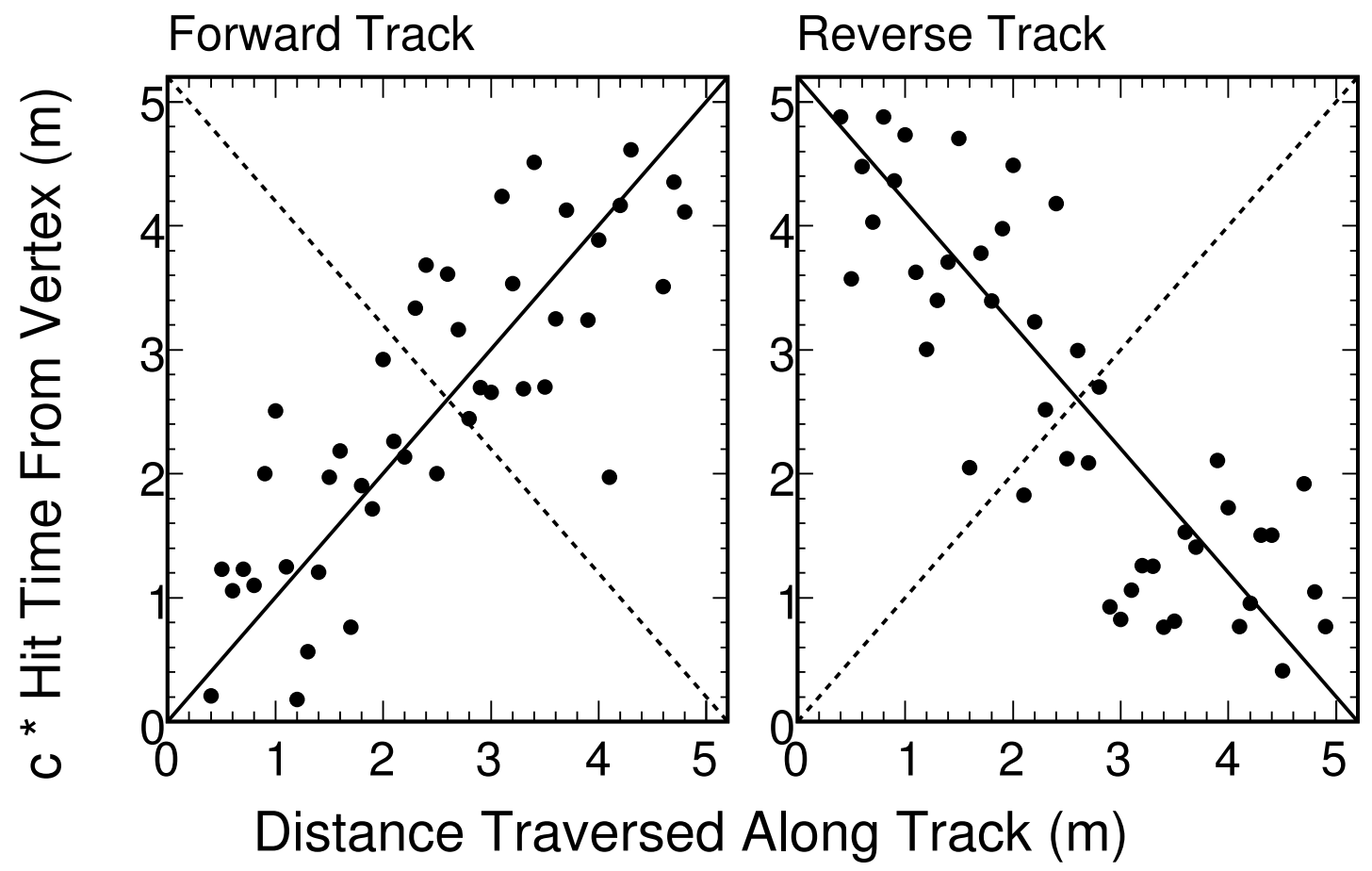

Figure 7.24: Two examples of how timing fits are used to determine the track direction

(a) Vertex vs End Timing

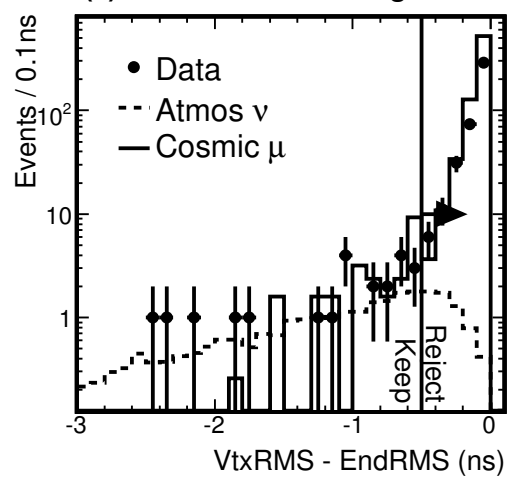

(b) Atmospheric $v$ Efficiency

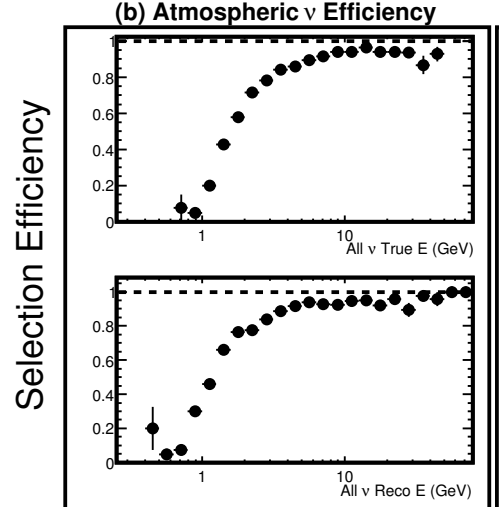

(c) Cosmic-Ray $\mu$ Efficiency

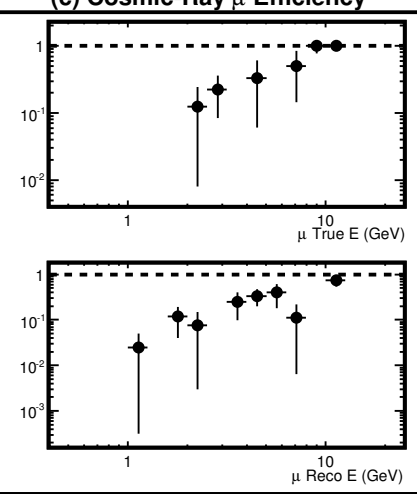

Figure 7.25: Comparison of Upward and Downward fits for PCUP Track-like events. 
with FC / PCDN track-like events, since the shield can not be used to reduce the background further for PCUP track-like events.

\section{- Vertex Hits Topology -}

The selection of FC and PCDN track-like events used the maximum 3D displacement of a hit from the track vertex in the 10 plane window around the track vertex (VtxRMax). For PCUP track-like events with TrkPlanes $\leq 20$, it is required that $V t x R M a x<1.25$ $\mathrm{m}$, which is the same requirement as was made in the selection of FC / PCDN track-like events.
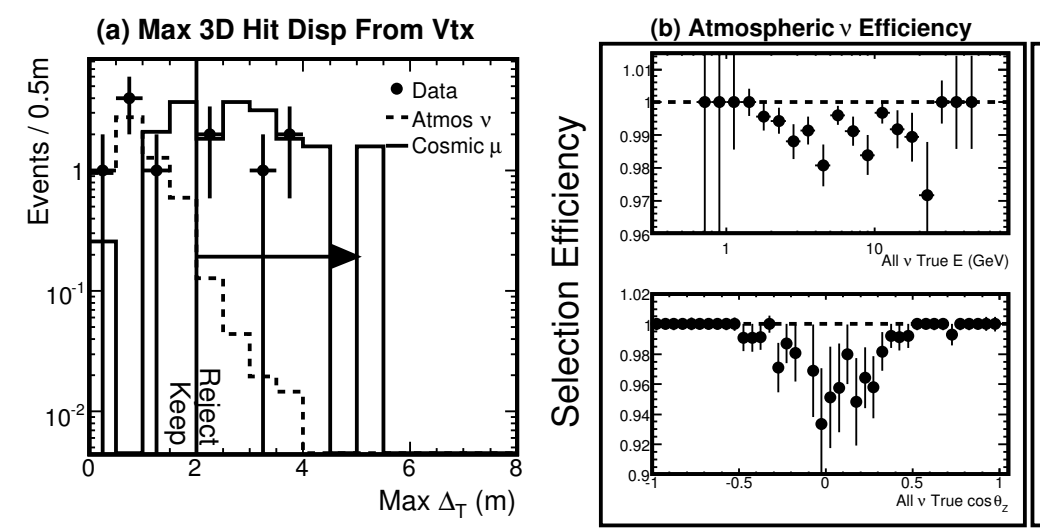
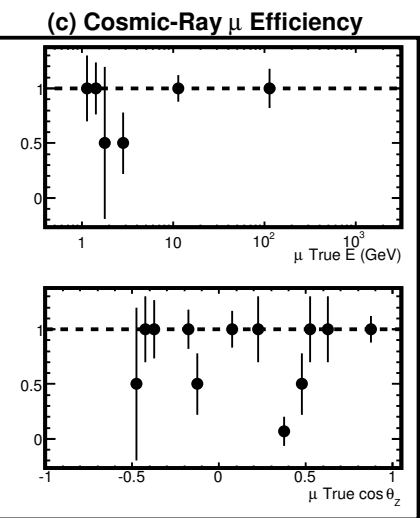

Figure 7.26: Maximum 3D displacement around the track vertex of all hits with $\geq 2$ pe for PCUP track-like events.

\section{- Track Timing -}

The $R M S_{\text {up }}$ and $R M S_{\text {down }}$ are measured by making the assumption that the particle has the speed of light. Once the direction is determined, the speed of the particle is measured by a charge weighted linear least squares fit to the mean hit position versus hit time. The resulting speed is another measurement of the timing quality for the event. The distribution of $\frac{1}{\beta}=\frac{c}{v}$ in Figure 7.27 a has a peak at 1.0 for the speed of light. The events with a reconstructed speed that is different from the speed of light by a factor of 2 are discarded (i.e. by requiring that $0.5<1 / \beta<2.0$ ).

The final selection of PCUP track-like events uses the $R M S_{u p}$ as depicted in Figure 7.27.b. By requiring that $R M S_{u p}<1.33 \mathrm{~ns}$, the number of expected cosmic-ray muon 
(a) Track Upward Time Slope

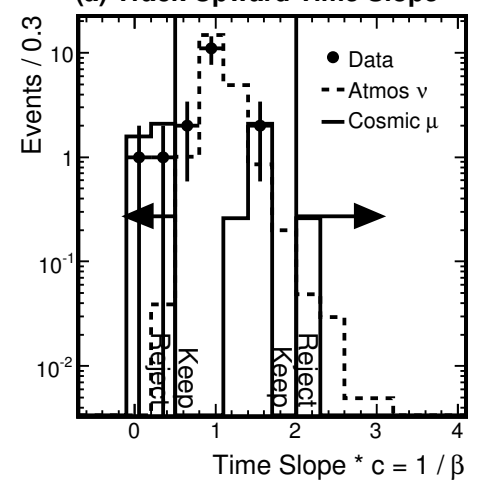

(b) Vertex Timing Quality

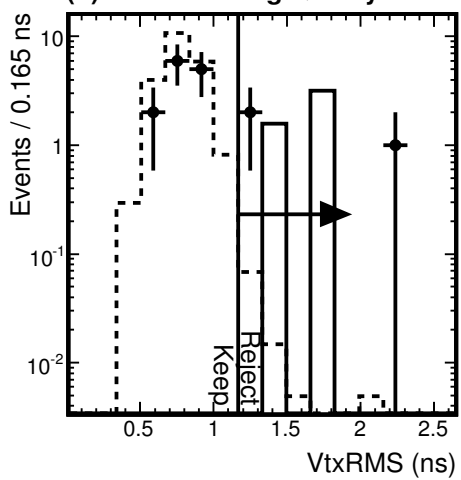

(c) Atmospheric $v$ Efficiency

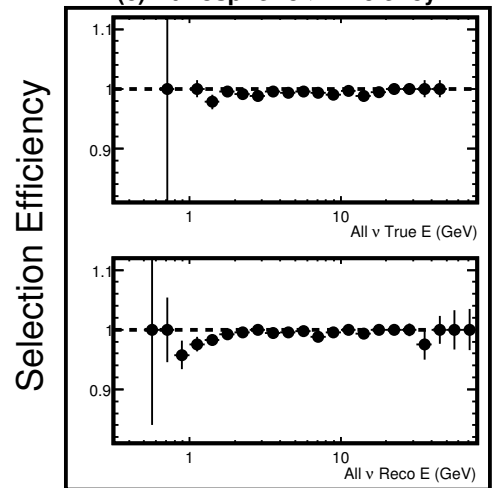

Figure 7.27: Timing resolution for PCUP Track-like events measured by the (a) time slope and (b) $R M S_{u p}$.

background events in the PCUP track-like sample is reduced to zero. Figure [7.27. c shows that the selection based on track timing has the effect of reducing the contribution of atmospheric neutrino interactions with low reconstructed energy, but not with low true energy. The result is a reduction in the number of events with a lower reconstructed energy than truth dictates.

\section{- Selection Results -}

The final set of PCUP Track-like events is shown in Table 17.11 The complete set of the data events selected in this category is listed in detail in Section A.2.3. The final set contain no expected background cosmic-ray muon events; i.e., the expected number of cosmic-ray muon is $<0.411$ with $68 \%$ confidence.

\begin{tabular}{|c|c|c|c|c|c|c|}
\hline & \multicolumn{6}{|c|}{ Monte Carlo } \\
& Data & $\stackrel{(-)}{\nu}_{\mu}$ CC & $\stackrel{(-)}{\nu}_{e}$ CC & $\nu$ NC & CR $\mu$ & CR N \\
\hline Clean PCUP Tracks & 435 & 27.9 & 0.093 & 0.11 & 416 & 0.16 \\
+ Upward Direction Resolution & 22 & 22.0 & 0.039 & 0.034 & 17 & 0 \\
+ Vertex Hits Topology & 17 & 21.8 & 0.034 & 0.034 & 4.76 & 0 \\
+Track Timing & 14 & 21.7 & 0.029 & 0.029 & 0 & 0 \\
\hline
\end{tabular}

Table 7.11: Number of Partially-contained upward track-like events through track timing selections. 


\subsection{Veto Shield}

Reconstructed tracks and showers are both created with a 4 dimensional vertex (3D space + time). The veto shield can be used to determine which of these tracks and showers are caused by cosmic-ray muons by identifying the shield strip hits that coincide with an event vertex temporally and spatially. All showering events will use the shield to identify any cosmic-ray muons, but only PCDN and FC track-like will use the shield for background reduction. First, all shield hits are matched to the vertex time, and then those strips that are matched in time are compared spatially to the vertex. For PCDN track-like events, the track vertex will always be examined with the shield planks (group of 8 strips in the veto shield). However, for FC track-like events, either the track vertex or track end may be examined with the shield planks. The procedure that determined which side of the track in a FC track-like event was used was outlined in Section 7.5.2.

The recorded timing information for the shield hit is the time that the signal was read out at the PMT. This time has to be corrected for the propagation time of the light from the point where the particle passed through the strip to the PMT. To match

the timing of a individual hit in the shield with an event in the detector, the following progression of time corrections is made:

1. Start with the calibrated time of a strip end (treat each end of the strip independently)

2. Subtract the time that the light took to travel the WLS fiber pigtail in the module and the clear fiber in the cable connecting the module to the MUX box.

3. Subtract the time that the light would have taken to travel down the WLS fiber embedded in the shield strip. This is estimated by the difference between $\mathrm{Z}$ of event vertex and $\mathrm{Z}$ of each strip end.

With the timing of the shield strips calibrated to the timing of the event in the detector, strips can be retained or discarded for further examination. If a shield strip time is $\pm 100 \mathrm{~ns}$ from the event vertex time, then the shield strip is "in time". If a shield strip is considered in time with an event, then the vertex location of that event is tested against the position of the shield plank. 
Cosmic-ray muons that generate events in the detector almost always enter the MINOS detector cavern from above the point where a detector event occurs. This fact is exploited by constraining the location of shield strips that may be used to veto an event. The Y position of the shield strip must be no more than $10 \mathrm{~cm}$ lower than a track vertex or $20 \mathrm{~cm}$ lower than a shower vertex. The $\Delta Y$ value is greater for showers than for track because the process that geometrically locates the vertex of an event is less precise for showers than it is for tracks.

Matching the $\mathrm{Z}$ coordinate of an event and the set of in-time shield strips is a more complex procedure than was used to match the Y-coordinate. The X and Y location of the shield strips are known within a few centimeters, but $\mathrm{Z}$ location has an ambiguity of $8 \mathrm{~m}$ due to the length of a shield strip. Unlike the detector, there aren't alternating layers of perpendicular strips to resolve this ambiguity.

If the event vertex falls directly under the $8 \mathrm{~m} \mathrm{Z}$ extent of a shield strip, then that strip is matched to the event in the Z-coordinate. A window in $\mathrm{Z}$ is also established around the plank in case a muon enters at an angle such that it may hit a shield plank and interact in a portion of the detector that is not under that shield plank. Events that occur deeper in the detector are less likely to have come from a particle that could hit a plank that is not above the event. Similarly, a shield plank that is closer to the detector edge is less likely to be hit by a muon that would create an event not under that plank. The window size it based on the radial position of the event vertex, and the radial position of the shield plank.

The event vertex radius is $E v V t x R O u t=\sqrt{V t x_{X}{ }^{2}+V t x_{Y}{ }^{2}}$, and the shield plank radius is PlankROut $=\sqrt{\text { Plank }_{X}^{2}+\text { Plank }_{Y}{ }^{2}}$. If the detector edge is assumed to be $4.0 \mathrm{~m}$ radially distant from the center, similar triangles can be used to devise an acceptable Z-window for each plank. The gap between planes is $5 \mathrm{~cm}$, and a gap of $N_{\text {Planes }}$ would have a width of $N_{\text {Planes }} * 5 \mathrm{~cm}$. Equation 7.7 is the solution for the $\mathrm{Z}$ window size from the similar triangle method for a particle to hit at PlankROut and travel through $N_{\text {Planes }}$ before making an event at $V t x R O u t$. Due to the poorer vertex location performance of the shower finder than the track finder, this algorithm uses $N_{\text {planes }}=1$ for tracks and $N_{\text {planes }}=3$ for showers.

$$
Z_{\text {Window }}=N_{\text {planes }} * 0.05 * \frac{\text { PlankROut }- \text { VtxROut }}{4.0 m-\text { VtxROut }}
$$


If at least one strip with at least 1 pe matches the time, $\mathrm{Y}$ location, and $\mathrm{Z}$ location criteria, then the event is vetoed. The performance of this veto procedure is quantified by the fraction of cosmic-ray muons that are vetoed from the data. This fraction is the "shield efficiency" $(\epsilon)$. The fraction of atmospheric neutrino interactions that are accidentally vetoed is the "shield inefficiency" $(\eta)$.

The number of events in a clean data sample $\left(N_{\text {Data }}\right)$ and the number of vetoed events in the same sample $\left(N_{\text {Vetoed }}\right)$ can be used to measure the shield efficiency or inefficiency if the number of cosmic-ray muons $\left(\mathcal{N}_{\mu}\right)$ and atmospheric neutrino interactions $\left(\mathcal{N}_{\nu}\right)$ are well known. For these quantities, and others described in this section, $N_{\text {type }}$ will refer to an observed number of events, and $\mathcal{N}_{\text {type }}$ will refer to an expect number of events derived from a simulated sample that is scaled to the data exposure analyzed in this thesis. It is important to distinguish between observed and expected number of events, as they treated statistically different.

$$
\begin{aligned}
N_{\text {Data }} & =\mathcal{N}_{\mu}+\mathcal{N}_{\nu} \\
N_{\text {Vetoed }} & =\epsilon * \mathcal{N}_{\mu}+\eta * \mathcal{N}_{\nu} \\
N_{\text {Signal }}=N_{\text {Data }}-N_{\text {Signal }} & =(1-\epsilon) * \mathcal{N}_{\mu}+(1-\eta) * \mathcal{N}_{\nu}
\end{aligned}
$$

Equation 7.8 and 7.9 demonstrate how these event counts are related, and tied in with the shield efficiency and inefficiency. The events that remain after the shield selection are labeled "signal" events, and Equation [7.10] shows how the quantity of signal events relates to the other event counts.

\subsubsection{Shield Inefficiency}

In order to measure the shield efficiency, there should be a reasonable estimate of the atmospheric neutrino interaction contribution to a data sample. Although this number is small, and the effect is minor, this will be taken in to account in the interest of completeness. In order to be able to measure the atmospheric neutrino interaction contribution to set of data events, and the subset of those events that are vetoed, the inefficiency must be measured first. 
The accidental veto of an atmospheric neutrino interaction is usually the result of natural $\gamma$ radiation in the cavern activating a shield strip, and the coincidence of that hit with a neutrino interaction. To measure the inefficiency, a set of shield triggers was generated by inverting the noise filter on raw data to obtain a sample of events induced by the background radiation.

Each of these shield triggers was tested against an atmospheric neutrino interaction. The selected set of simulated showering and track-like events are randomly overlaid with a shield trigger. The set of shield strip was tested against the event to match the veto criteria. In this situation, the value of $N_{\text {Data }}$ is the number of simulated atmospheric neutrino interactions tested, or $N_{D a t a}=N_{\nu}$ and $N_{\mu}=0$. Combining this with equations 7.8 and 7.9 the inefficiency is given by the ratio of the number of vetoed events to the full data sample $\left(\eta=N_{\text {Vetoed }} / N_{\text {Data }}\right)$.

Using this procedure independently for the set of showering and track-like events, the inefficiencies are measured to be $\eta_{s h w}=2.71 \pm 0.02 \%$ and $\eta_{t r k}=2.55 \pm 0.02 \%$. The errors quoted are statistical, and the measurement of the inefficiency is also subject to some systematic errors. The following systematic effects have been examined:

- Atmospheric Set - The final selected sets of simulated atmospheric neutrinos were used to measure the inefficiency. The same experimental measurement of the inefficiency is run again for both samples with set of events that remain after the data quality and vertex containment selections.

- Random Seed - In the overlay process, there are many more data shield triggers than simulated atmospheric neutrino events. The set of simulated atmospheric neutrinos is shuffled, and each is overlain on a sequential data shield trigger. When the simulated atmospheric neutrino set is exhausted, the set is shuffled again and the data shield trigger sequence continues with the reshuffled set. To measure the systematic effect of the randomizing shuffle, the experiment is run twice with different random seeds.

- Shield Section - The shield is divided into four section, with two sections over each supermodule. All of the four section are geometrically and electronically identical, and so all four are expected to have a similar response. In order to 
verify this, a comparison is made amongst the four shield sections of the rate at which each section uniquely vetoes events.

- Seasonal Variation - Over the course of the run, there were times of higher background shield activity due to seasonal effects on the flux of cosmic-ray muons. The inefficiency is measured over the run time span in two week intervals to examine systematic variation. Figures $7.28 \mathrm{a}$ and $7.28 \mathrm{~b}$ show the inefficiency measured in two week intervals for showering and track-like events respectively. The mean and RMS of the efficiencies (weighted by $1 / \sigma^{2}$ ) from Figures [7.28, and $\mathrm{b}$ are shown in Figures 7.28 , c and d respectively.
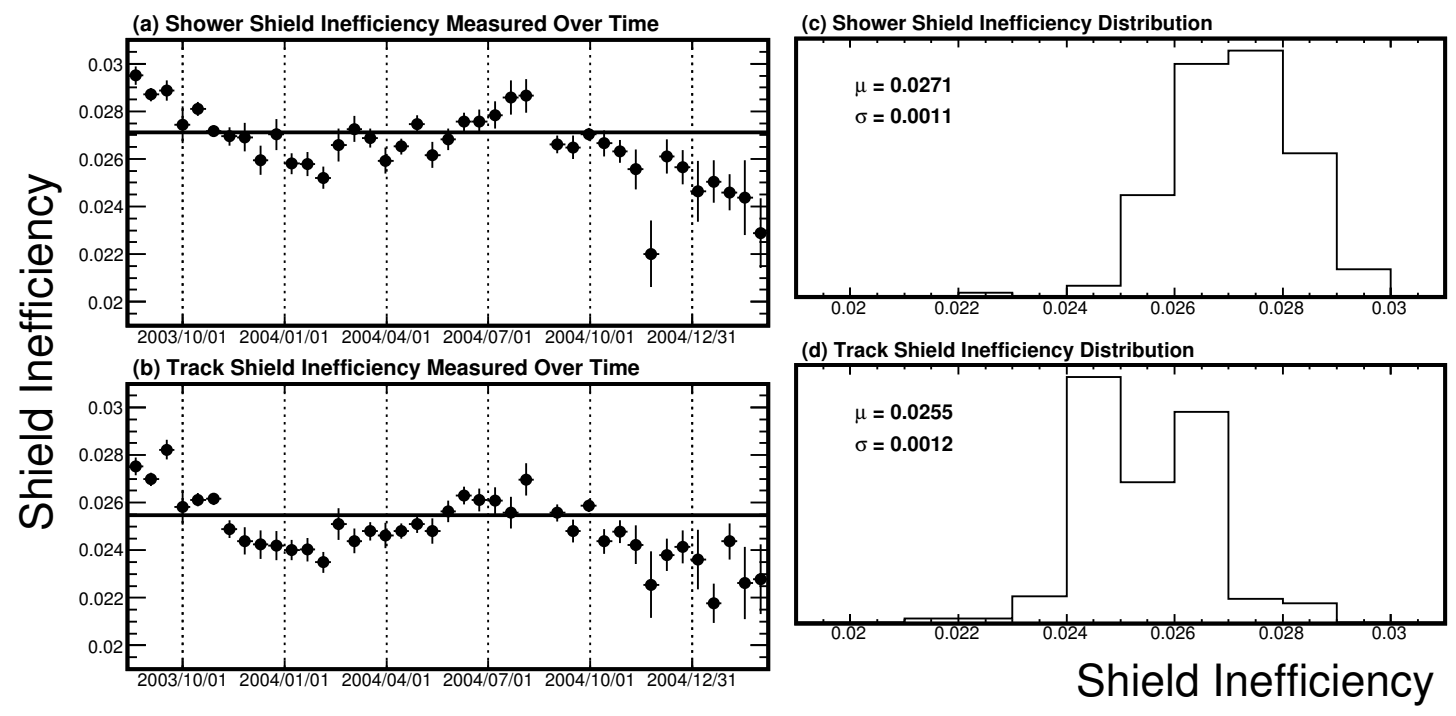

Figure 7.28: Shield inefficiency measured for showering and track-like events over the course of the data collection

\begin{tabular}{|c|c|c|}
\hline Systematic Error & $\Delta \eta_{\text {trk }}$ & $\Delta \eta_{\text {shw }}$ \\
\hline Atmospheric Set & $0.03 \%$ & $0.00 \%$ \\
Random Seed & $0.01 \%$ & $0.01 \%$ \\
Shield Section & $0.05 \%$ & $0.05 \%$ \\
Seasonal Variation & $0.11 \%$ & $0.12 \%$ \\
\hline Cumulative Error & $0.13 \%$ & $0.13 \%$ \\
\hline
\end{tabular}

Table 7.12: Effect of systematic changes on the measurement of the shield inefficiency 
The results of these systematic variations are shown in Table 7.12 The resulting measurements of the shield inefficiency are $\eta_{s h w}=2.71 \pm 0.02$ (stat.) $\pm 0.13($ syst.) $\%$ and $\eta_{\text {trk }}=2.55 \pm 0.02$ (stat. $) \pm 0.13$ (syst.) $\%$.

\subsubsection{Shield Efficiency}

To measure the inefficiency, the case was used where $N_{\text {Data }}=N_{\nu}$ and therefore $N_{\mu}=0$. In order to measure the efficiency directly from the data, it possible to reach a situation where the data sample can be described by $N_{\nu}<<N_{\mu}$. Such a sample is achieved by removing all of the selection criteria except for containment and data quality, and measuring the rate at which events are vetoed. This is a straight-forward method that measures the efficiency with a large set of data, and thus has a small statistical error. The efficiencies measured with this method are $\epsilon_{s h w}=97.58 \pm 0.02 \%$ and $\epsilon_{\text {trk }}=$ $97.43 \pm 0.06 \%$. However, this method is deficient in that it does not measure the shield performance for the type of track-like or showering event that has been selected as a neutrino candidate.

To study this further, the topological cuts are loosened enough so that the neutrino signal in the data is overwhelmed by cosmic-ray background. The final selections are also inverted to reduce the number of expected neutrinos in the sample. The resulting measurements of the shield efficiency are now $\epsilon_{s h w}=97.6 \pm 0.3 \%$ and $\epsilon_{t r k}=97.3 \pm 0.2 \%$, which are consistent with previous measurement of the shield efficiency.

The errors expressed on the previous measurements of the shield efficiency were exclusively statistical errors. The systematic errors were examined as described earlier.

\begin{tabular}{|c|c|c|}
\hline Systematic Error & $\Delta \epsilon_{\text {trk }}$ & $\Delta \epsilon_{\text {shw }}$ \\
\hline Shield Section & $0.2 \%$ & $0.3 \%$ \\
Seasonal Variation & $0.4 \%$ & $0.2 \%$ \\
\hline Cumulative Error & $0.4 \%$ & $0.4 \%$ \\
\hline
\end{tabular}

Table 7.13: Effect of systematic changes on the measurement of the shield inefficiency

Including the systematic errors, the measured values of the shield efficiency are $\epsilon_{\text {shw }}=97.6 \pm 0.3$ (stat.) \pm 0.4 (syst.) $\%$ and $\epsilon_{\text {trk }}=97.3 \pm 0.2$ (stat.) $\pm 0.4($ syst. $) \%$. As in the shield inefficiency measurement, the systematic error dominates the statistical error. In both cases, the seasonal variation contributes a large error; this would be difficult to 

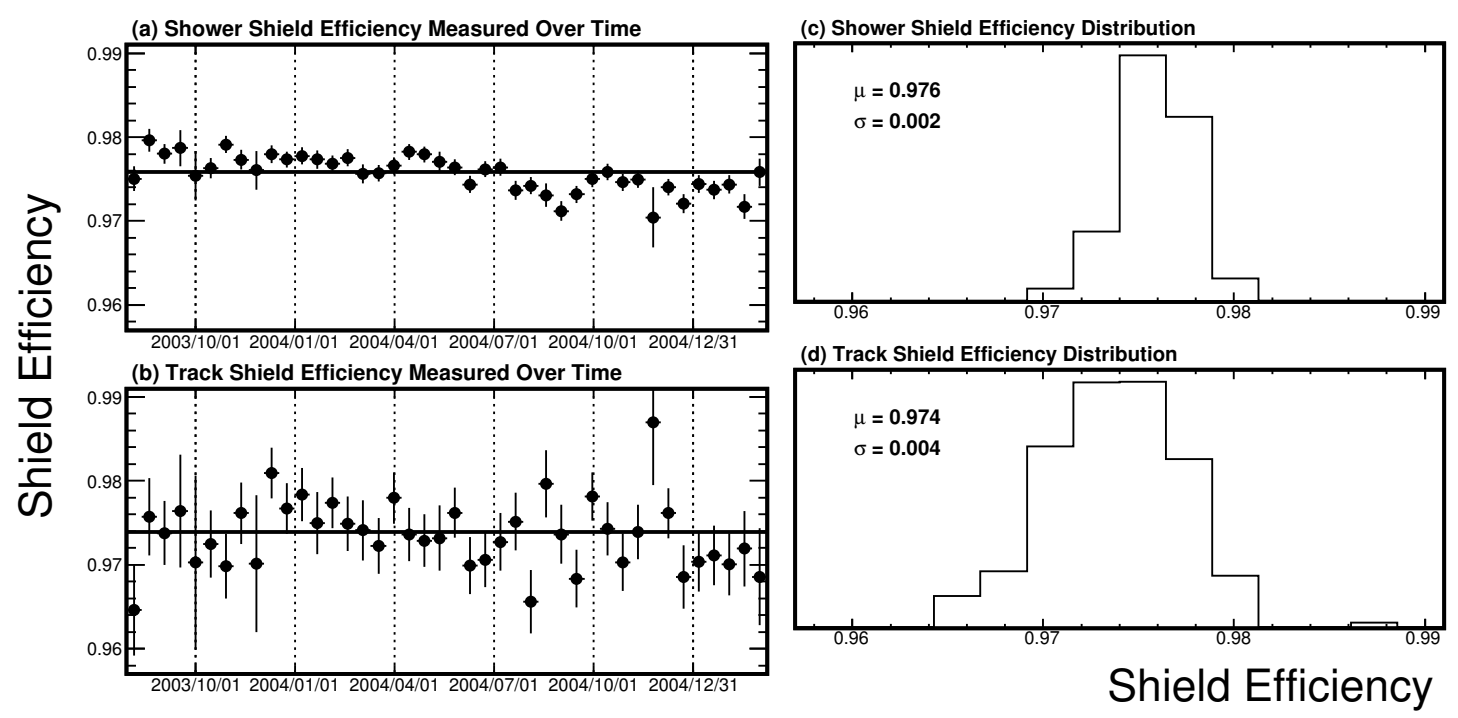

Figure 7.29: Shield efficiency measured for showering and track-like events over the course of the data collection

resolve. The error between the shield sections however could be resolved by applying the shield veto in sections. Both of the predominant contributing errors to systematic error could be abated by adjusting the veto shield electronic response. The balance of shield efficiency and inefficiency systematic errors is an essential topic for the future of the atmospheric neutrino analysis, and warrants further investigation.

\subsection{Final Data Set}

The showering and track-like FC and PCDN events use the veto shield to enhance the selection, and in section [7.6 the efficiency and inefficiency of the veto shield selection was measured. Equations [7.8 and [7.9] are used to find the number of signal events. Equation 7.12 expresses the signal size with constituents of only $\mathcal{N}_{\nu}$ and $\mathcal{N}_{\mu}$. The deficiency with Equation $\mathbf{7 . 1 2}$ is that the size of the cosmic-ray muon contribution is not well known from the cosmic-ray simulation sample. The low energy end of the cosmic-ray muon spectrum $\left(E_{\mu}<2 \mathrm{GeV}\right)$ makes a significant contribution to the background in the atmospheric neutrino sample, and this portion of the spectrum has a large theoretical uncertainty. 


$$
\begin{aligned}
E_{\text {Signal }} & =\frac{\epsilon-\eta}{\epsilon} \mathcal{N}_{\nu}+\frac{1-\epsilon}{\epsilon} N_{\text {Vetoed }} \\
N_{\nu} & =\frac{\epsilon}{\epsilon-\eta} N_{\text {Signal }}-\frac{1-\epsilon}{\epsilon-\eta} N_{\text {Vetoed }}
\end{aligned}
$$

This deficiency is resolved by solving for $N_{\text {Signal }}$ in terms of $\mathcal{N}_{\nu}$ and $N_{\text {Vetoed }}$, as seen in Equation 7.12. This equation yields the expected number of signal events $\left(E_{\text {Signal }}\right)$ to compare to the observed number of signal events. $\mathcal{N}_{\nu}$ is known from a large sample of simulated atmospheric neutrinos, and $N_{\text {Vetoed }}$ is measured from the data. By virtue of the high efficiency $(\epsilon \rightarrow 1.0)$ and low inefficiency $(\eta \rightarrow 0.0)$, the multiplicative factors for the $N_{\nu}$ in Equations $7.12(1-\eta)$ and $7.12\left(1-\frac{\eta}{\epsilon}\right)$ are virtually equivalent. Equation 7.12 can rearranged into Equation [7.13, which yields a measured number of neutrino interaction events $\left(N_{\nu}\right)$ to compare to the expected number of neutrino interactions from the Monte Carlo set $\left(\mathcal{N}_{\nu}\right)$. The form of Equation 7.13 becomes $N_{\nu}=N_{\text {Signal }}$ for a perfectly efficient shield selection $(\epsilon \rightarrow 1.0$ and $\eta \rightarrow 0.0)$.

Table 7.14 shows the final numbers of atmospheric neutrino candidates in the showering and track-like samples. The events types that also use the shield to enhance selection have a second line with the results after the veto selection. On the veto shield line, the simulated event yields are scaled by the expected reduction derived from the shield efficiencies. The simulated neutron yield is scaled by the same factor as the simulated atmospheric neutrino yield, as it is assumed that neutrons that interact deep in the detector will not have caused hits in the shield. The Cosmic $\mu$ column in the "Data" group contains the expected number of cosmic-ray muons that contaminate the signal sample, derived from the shield efficiency and the number of vetoed events.

The two cosmic $\mu$ columns are expected to have equivalent values, but this not the case for the showering event sample. The simulated cosmic-ray muons that are selected as showering neutrino candidates are mostly $(>80 \%)$ in the lower energy portion of the spectrum where the cosmic ray muon flux is poorly understood.

\subsubsection{Double Ratio}

The double ratio measured in this thesis $(\mathcal{R})$ is the ratio of the ratio of the observed

number of selected track-like events over showering events $\left(R_{T r k / S h w}^{d a t a}\right)$ over the ratio of 


\begin{tabular}{|c|c|c|c|c|c|}
\hline & \multicolumn{2}{|c|}{ Data } & \multicolumn{3}{c|}{ Monte Carlo } \\
Event Sample & Events & Cosmic $\mu$ & Atmos. $\nu$ & Cosmic $\mu$ & Neutrons \\
\hline Sel. Showering & 244 & - & $84.1 \pm 0.6$ & $74.5 \pm 11.3$ & $2.42 \pm 0.6$ \\
+ Veto Shield & 89 & $3.81 \pm 0.58$ & $81.8 \pm 0.6$ & $1.78 \pm 0.35$ & $2.36 \pm 0.6$ \\
\hline \hline Sel. FC Track-like & 229 & - & $102.1 \pm 0.7$ & $126.0 \pm 12.4$ & 0 \\
+ Veto Shield & 72 & $4.19 \pm 0.60$ & $99.4 \pm 0.7$ & $4.45 \pm 0.70$ & 0 \\
\hline \hline Sel. PCDN Track-like & 50 & - & $22.9 \pm 0.3$ & $44.4 \pm 8.6$ & 0 \\
+ Veto Shield & 25 & $0.67 \pm 0.16$ & $22.2 \pm 0.3$ & $1.15 \pm 0.26$ & 0 \\
\hline \hline Sel. PCUP Track-like & 14 & - & $21.7 \pm 0.3$ & 0 & 0 \\
\hline
\end{tabular}

Table 7.14: Final numbers of selected showering and track-like events, including veto selection.
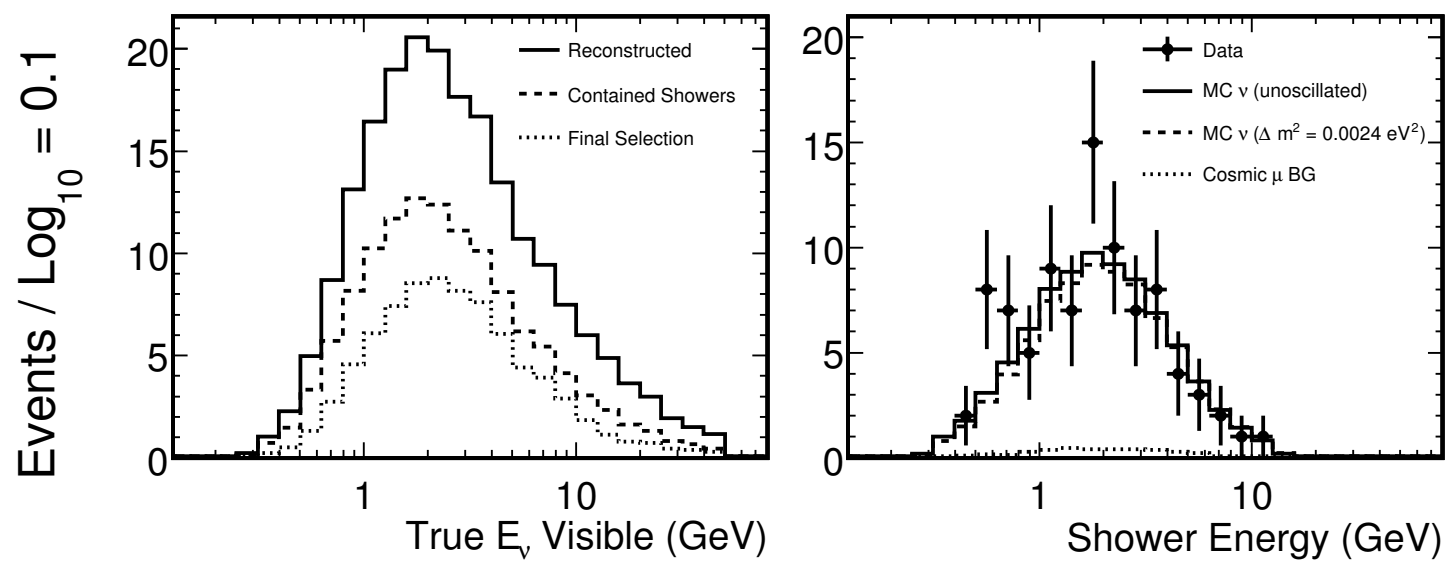

Figure 7.30: True and reconstructed energy distributions of selected showering events.

the expected number of selected track-like events over showering events $\left(R_{\text {Trk/Shw }}^{M C}\right)$, as expressed in equation 7.14 .

$$
\begin{aligned}
\mathcal{R} & =\frac{R_{\text {Trk } / S h w}^{\text {data }}}{R_{\text {Trk } / S h w}^{M C}} \\
& =0.74_{-0.10}^{+0.12}
\end{aligned}
$$

The null oscillation hypothesis double ratio of 1.0 is disfavored with a $98.8 \%$ single sided confidence limit (97.4\% double-sided confidence). The derivation of the statistical error of the double ratio is discussed in more detail in Appendix Section B.1 There 
(a) Selected Showering Events: Event Date

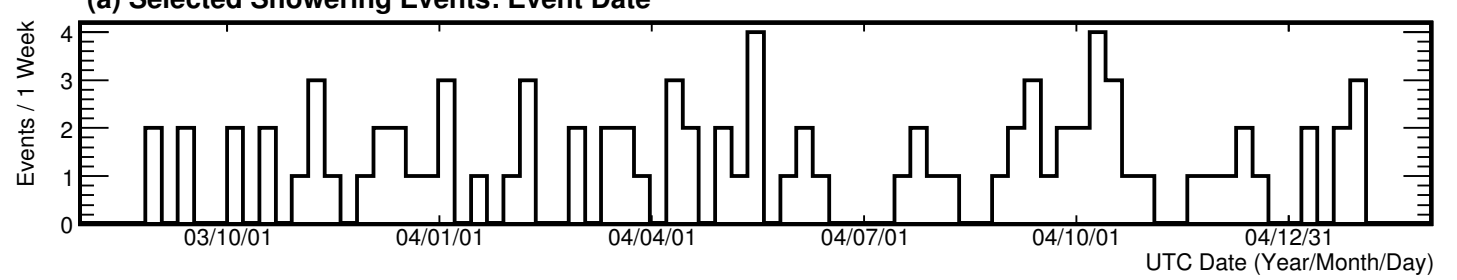

(b) Vertex X v Y Position

(c) Vertex Depth Into Detector

(d) Vertex Z Position
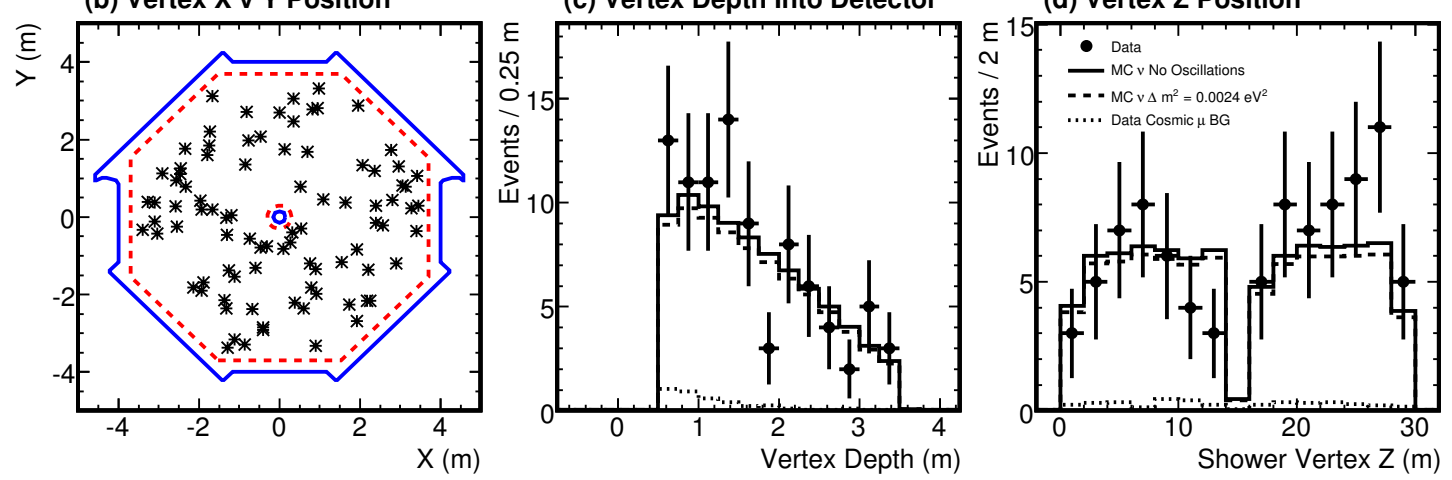

Figure 7.31: Temporal and spatial distributions of selected showering events.

(a) Selected Track-like FC Events: Event Date

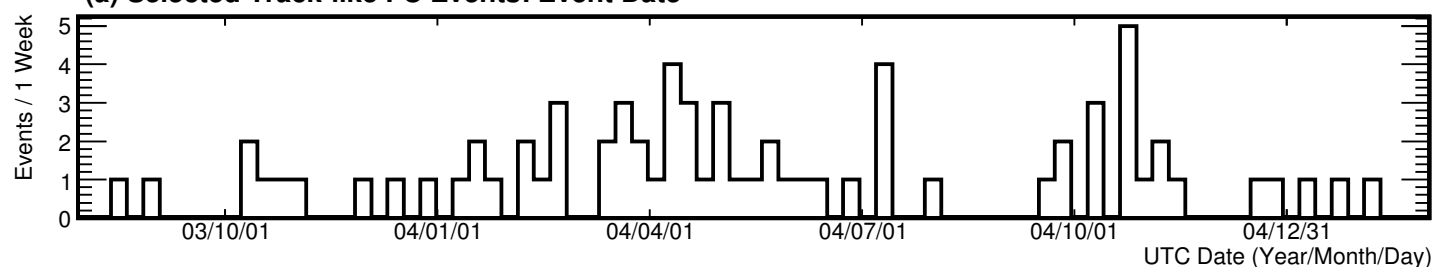

(b) Vertex X v Y Position

(c) Vertex Depth Into Detector
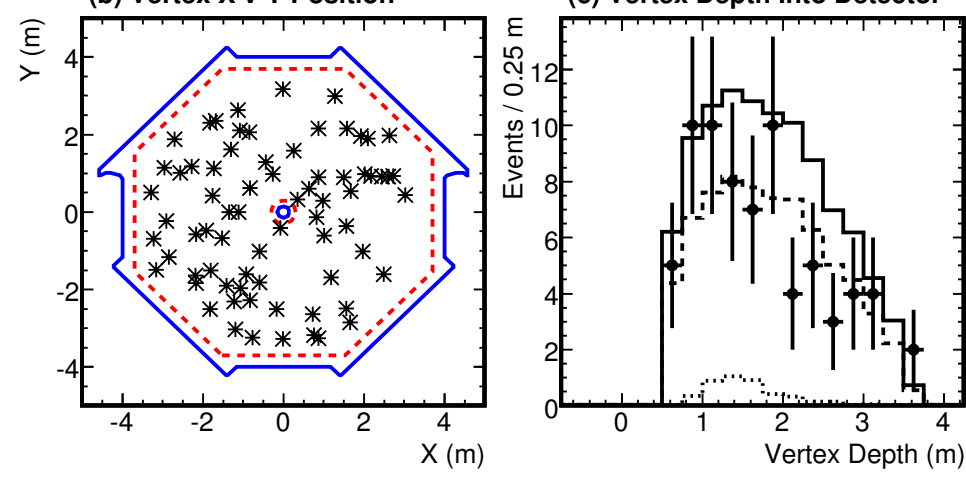

(d) Vertex Z Position

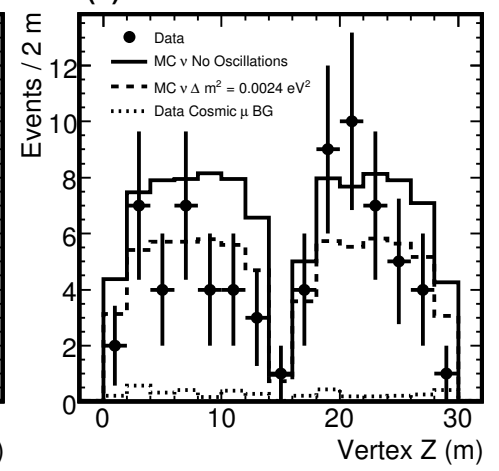

Figure 7.32: Temporal and spatial distributions of selected track-like FC events. 
(a) Selected Track-like PCDN Events: Event Date

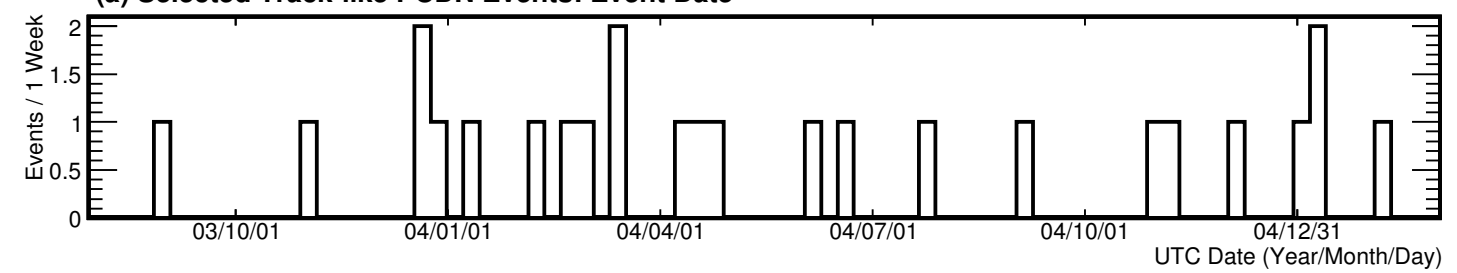

(b) Vertex X v Y Position

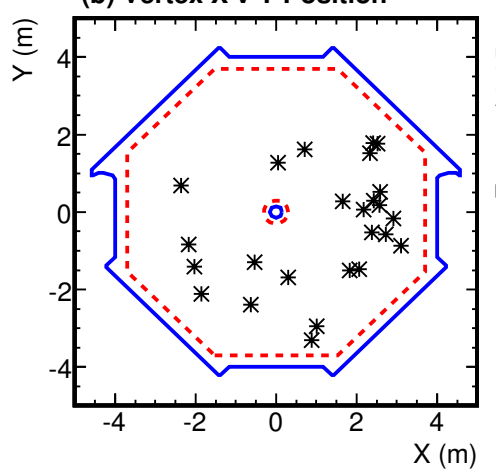

(c) Vertex Depth Into Detector

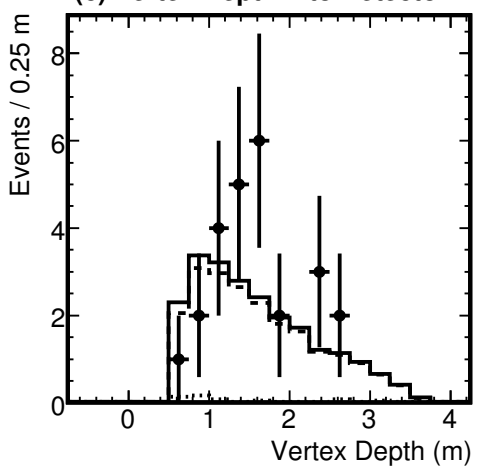

(d) Vertex Z Position

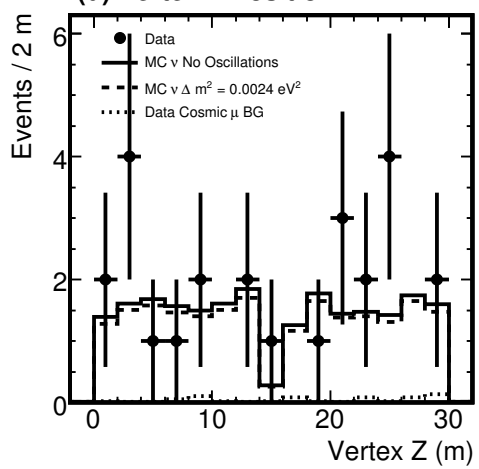

Figure 7.33: Temporal and spatial distributions of selected track-like PCDN events.

(a) Selected Track-like PCUP Events: Event Date

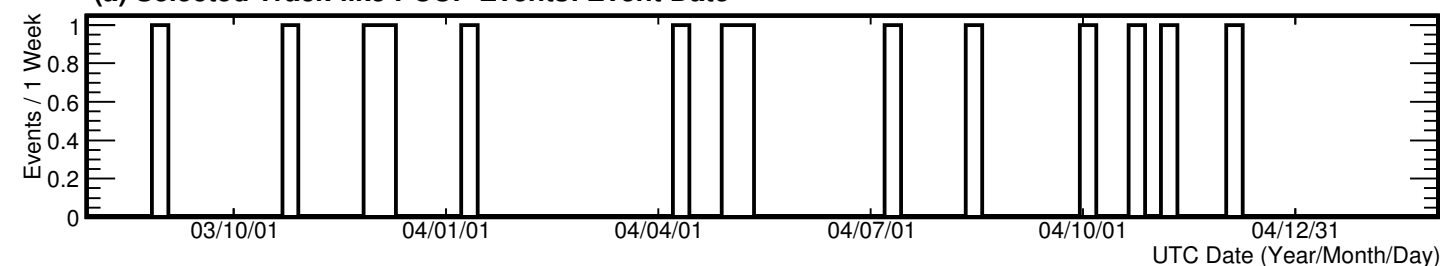

(b) Vertex X v Y Position

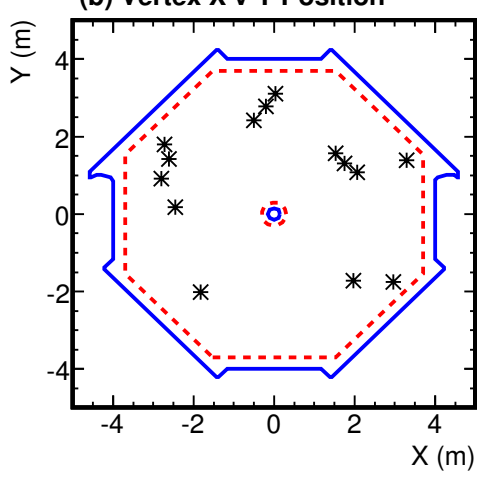

(c) Vertex Depth Into Detector

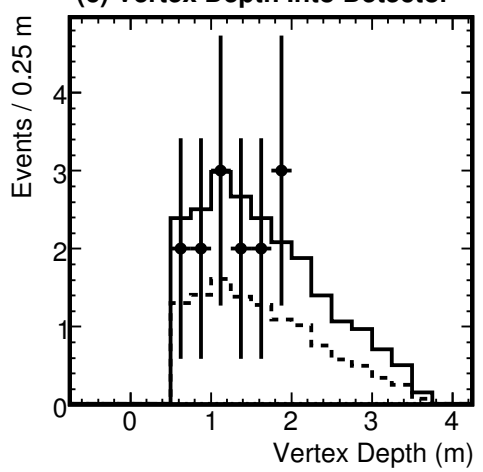

(d) Vertex Z Position

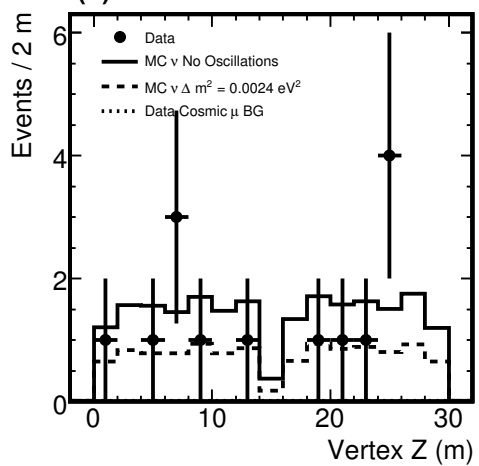

Figure 7.34: Temporal and spatial distributions of selected track-like PCUP events. 
are several uncertainties in the simulation of atmospheric neutrinos [6] that will lead to systematic errors. The following systematic variances are applied to the simulated sets to measure the systematic error.

- Atmospheric Neutrino Flux Scale - One purpose of using the double ratio of showering and track-like events is to evaluate oscillation while reducing the effect from the atmospheric neutrino flux uncertainty, but there still exists a background muon contribution that could allow this to contribute on some level. To study this, the overall flux has been varied by up to $\pm 20 \%$.

- Atmospheric Neutrino Flavor Ratio - The Bartol model predicts the ratio of $\nu_{e} / \nu_{\mu}$ flux with a $1 \%$ uncertainty for $E_{\nu}<10 \mathrm{GeV}$. [6]. This uncertainty directly contributes a $1 \%$ uncertainty to the double ratio.

- Energy Tracking Cut-Off - The primary simulation follows particles through the detector as they lose energy until they reach $10 \mathrm{keV}$, at which point they are discarded, in the interest of computing expedience. An additional simulation set that uses a $10 \mathrm{keV}$ cut-off has been used to examine this systematic variance.

- Quasi-elastic cross section - The cross section for quasi-elastic (QE) neutrino interactions has an uncertainty of $\sim 10 \%$ for neutrino energies less than $10 \mathrm{GeV}$ (the dominant energy range for the selected atmospheric neutrinos). The $\mathrm{QE}$ events in both samples have their weight changed by $\pm 10 \%$ to examine the effect of this uncertainty.

- Neutral-current cross section - The cross section for neutral-current (NC) neutrino interactions has an uncertainty of $\sim 20 \%$. The NC events in both samples have their weight changed by $\pm 20 \%$ to examine the effect of this uncertainty.

- Neutron Flux - The neutron flux scale is based on the scale obtained for the cosmic ray muon flux. The content of the cosmic-ray muons in the final sample is determined directly from the data with the shield efficiencies. The neutron contribution however relies on the yield from the simulated set. The neutron flux scale has been varied by $\pm 20 \%$ to examine the effect of this uncertainty. 


\begin{tabular}{|c|c|c|c|}
\hline Systematic & $\Delta$ Showers $(\%)$ & $\Delta$ Tracks $(\%)$ & $\Delta D R(\%)$ \\
\hline Flux Scale $\pm 20 \%$ & \pm 18.6 & \pm 19.3 & $\mp 0.715$ \\
10 keV Tracking Cut-off & +4.34 & +0.023 & +4.34 \\
Quasi-elastic x-sect $\pm 10 \%$ & \pm 2.31 & \pm 4.43 & $\mp 1.80$ \\
Neutral-current x-sect $\pm 25 \%$ & \pm 4.14 & \pm 0.452 & \pm 3.69 \\
Neutron Scale $\pm 20 \%$ & \pm 0.537 & 0 & \pm 0.537 \\
\hline Cumulative Error & 19.7 & 19.8 & 6.12 \\
\hline
\end{tabular}

Table 7.15: Effects from systematic changes of the experiment on the measurements of tracks and showers counts along with double ratio. The cumulative error is found by adding all error sources in quadrature.

Table 7.15 shows how these systematic changes affect both the expected number of showering events, track-like events, and how those would in turn affect the double ratio measurement. The double ratio is now more fully expressed as:

$$
\mathrm{R}=0.74_{-0.10}^{+0.12}(\text { stat. }) \pm 0.04 \text { (syst.) }
$$

The Monte Carlo simulation that determined this confidence limit was run again with the systematic errors included, and the double ratio was found to disfavor the null oscillation hypothesis with a $98.0 \%$ single-sided confidence limit (96.0\% doublesided confidence limit). The double ratio presents interesting information regarding the oscillation of atmospheric neutrinos, but doesn't quantify the oscillation parameters. In the next chapter, the double ratio is used to quantify oscillation parameters, and to measure the atmospheric neutrino flux.

\subsubsection{Oscillation Independent Flux Measurement}

Soudan2 presented a first-order measurement of the atmospheric-neutrino flux [3] made with the electron-neutrino interaction rate. They selected a set of $\nu_{e}$ CC-like events composed of an expected small fraction of $\nu_{\mu}$ CC interactions $(\sim 4 \%)$ and $\nu$ NC interactions $(\sim 3 \%)$, and predominantly $\nu_{e} \mathrm{CC}$ interactions, and used this to measure the flux scale for the Bartol flux to be $S_{a t m}=0.88 \pm 0.07$.

In this analysis, $O_{s h w}=89$ showering events were observed, where $E_{s h w}=87.9$ events are expected. The expected number of events is comprised of a number of neutrinos $\left(E_{\nu}\right.$ $=81.78)$, a number of cosmic muons $\left(E_{\mu}=3.81\right)$, and a number of neutrons $\left(E_{N}=2.36\right)$. 
The atmospheric scale factor can be measured based on this composition of showering neutrino interactions (shown in Equation [7.17) with out concern for oscillation, much as was done in Soudan2 to arrive at a scale factor of $0.88 \pm 0.07$ [3] This measurement results in:

$$
\begin{aligned}
S_{a t m} & =\frac{O_{\text {shw }}-E_{N}-E_{\mu}}{E_{\nu}} \\
& =1.01 \pm 0.12(\text { stat. }) \pm 0.07 \text { (syst.) }
\end{aligned}
$$

\begin{tabular}{|c|c|c|}
\hline Systematic & $S_{a t m}$ & $\Delta S_{a t m}(\%)$ \\
\hline 10 keV Cut-off & 0.97 & -4.46 \\
QE x-sect $\pm 10 \%$ & 0.98 & $\mp 2.83$ \\
& 1.04 & \\
NC x-sect $\pm 25 \%$ & 0.97 & $\mp 4.46$ \\
& 1.06 & \\
Neutron Flux $\pm 20 \%$ & 1.01 & $\mp 0.570$ \\
\hline Cumulative Error & & 6.03 \\
\hline
\end{tabular}

Table 7.16: Systematic errors for the measurement of the flux scale factor measured with the rate of showering atmospheric-neutrino interactions. The cumulative error is found by adding all error sources in quadrature.

The systematic errors described in Section [7.7.1 have been applied to the measurement of the flux scale as well (shown in Table [7.16). In addition, two alternate flux models have been investigated, with the intent of making a flux scale measurement using the same method above. The first alternate model uses the Battistoni atmospheric neutrino model presented by Battistoni, et.al. [38. The second alternate model uses a GHEISHA hadron interaction model [84], as opposed to the default GCALOR model 83]. The flux scale is measured for both of these models with all systematic errors mentioned above except the Energy Tracking Cut-Off. The resulting flux scale factors are:

$$
\begin{aligned}
S_{\text {atm }}: \text { Bartol } & =1.01 \pm 0.12(\text { stat. }) \pm 0.07 \text { (syst. }) \\
S_{\text {atm }}: \text { Battistoni } & =1.15 \pm 0.13(\text { stat. }) \pm 0.07 \text { (syst. }) \\
S_{\text {atm }}: \text { Gheisha } & =0.92 \pm 0.11(\text { stat. }) \pm 0.06(\text { syst. })
\end{aligned}
$$


This flux measurement method is lacking because the presumption has been made that the number of showering events is not affected by oscillation. It is reflected in Table 7.4 .5 that $\sim 20 \%$ of the showering events are expected to be caused by $\nu_{\mu} \mathrm{CC}$ interactions (without oscillation). In the next chapter, oscillation is examined with the combination of the track-like and showering event samples in order to measure the atmospheric neutrino flux including the effects of oscillation. 


\section{Chapter 8}

\section{Oscillation Analysis}

The previous chapters of thesis have presented the selection of showering and track-like atmospheric-neutrino interaction candidates. These sets have been used to measure an

atmospheric-neutrino flavor double ratio $\left(0.74_{-0.10}^{+0.12}(\right.$ stat. $\left.) \pm 0.04\right)$, and a set of flux scale factors that may be used as a normalization term in an oscillation analysis. In this chapter, atmospheric neutrino oscillation is explored in order to enhance the measurement of the atmospheric neutrino flux.

The atmospheric neutrino flux scale is measured with two different oscillation analyses. First, the double ratio is used to fit oscillation parameters with a frequentist method, and the same measurement in Section 7.7 .2 is repeated, where the expected number of showering events $\left(E_{s h w}\right)$ has been altered by the best fit oscillation parameters. Second, the number of showering and track-like events are used to fit oscillation parameters and the flux scale with a maximum likelihood method, and a best fit flux scale value emerges directly from the fit method.

\subsection{Neutrino Oscillation Procedure}

To analyze a set of atmospheric-neutrino interaction candidates for oscillation, individual neutrinos can be oscillated with a re-weighting procedure. The analyses in this chapter use a two-neutrino-oscillation formulation, with the assumption that muon neutrinos oscillate into tau neutrinos. The probability of a muon neutrino oscillating into a tau neutrino (derived in Section 2.3 ) is: 


$$
P\left(\nu_{\mu} \rightarrow \nu_{\tau}\right)=\sin ^{2}(2 \theta) \sin ^{2}\left(1.27 \frac{L \Delta m^{2}}{E}\right)
$$

Both the energy $(E)$ and distance traveled by the neutrino from the production point $(L)$ are needed to calculate the oscillation probability with Equation 8.1 The true energy and direction is known for a simulated neutrino that interacts with the detector. The neutrino propagation length is derived from the geometry in Figure 8.1

$$
L=\sqrt{(R-D)^{2} \cos ^{2}(\theta)+(D+H)(2 R-D+H)}-(R-D) \cos (\theta)
$$

Where:

$\mathbf{R}=$ Mean Radius of Earth $(6371.315 \mathrm{~km})$

$\mathbf{D}=$ Vertical Depth of Soudan Mine $(0.65 \mathrm{~km})$

$\mathbf{H}=$ Atmospheric Neutrino Production Height $(25 \mathrm{~km})$

$\cos (\boldsymbol{\theta})=$ Neutrino Zenith Angle $\left(\theta_{\text {zenith }}\right)$ at Detector Site

A value of $\frac{L}{E}$ is known for each simulated neutrino, and, combined with a pair of oscillation parameters $\left(\sin ^{2}(2 \theta), \Delta m^{2}\right)$, an oscillation probability is also known for a each simulated neutrino. Using the assumption that $\nu_{\mu} \rightarrow \nu_{\tau}$ is the sole oscillation process, all simulated $\nu_{\mu}$ CC interactions should be weighted by $\mathrm{P}$ from Equation 8.1. Additional sets of atmospheric neutrino have been generated (described in Section 6.3.1) of tau neutrinos. No tau neutrinos are expected to be seen in the primary atmospheric neutrino flux, and the generated neutrinos are the result of $100 \%$ oscillation. The $\nu_{\tau}$ $\mathrm{CC}$ interactions from this simulated sample is oscillated by a weighting of $1-P$, again using Equation 8.1. With this weighting scheme, muon neutrinos are reduced from the sample and tau neutrinos takes their place.

Both oscillation analyses examined in this chapter use a 2-D oscillation parameter grid in $\sin ^{2}(2 \theta), \Delta m^{2}$ space with dimensions spanning $0.0<\sin ^{2}(2 \theta)<1.0$ and $10^{-5} \mathrm{eV}^{2}<\Delta m^{2}<10^{1} \mathrm{eV}^{2}$, and binned $100 \times 100$. The re-weighting procedure described above is performed with the oscillation parameters at each grid point. The oscillation analyses then fit the two parameters with a grid search fitting method. 


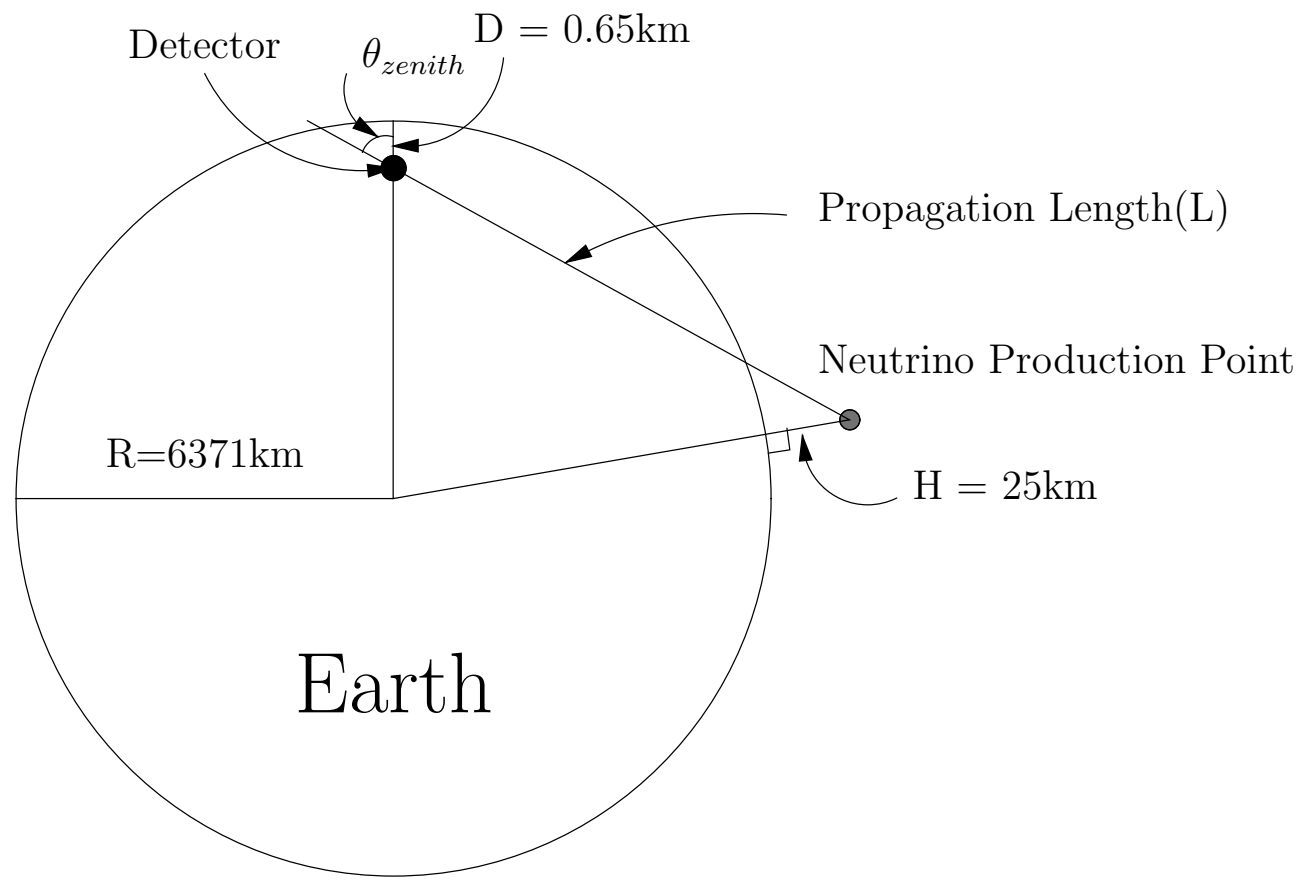

Figure 8.1: A drawing of Earth (not to scale), where the small filled black circle represents the detector in the mine, and the gray circle represents the production point of a neutrino in the atmosphere, $25 \mathrm{~km}$ above Earth's surface. 


\subsection{Frequentist Double Ratio Oscillation Analysis}

The double ratio was measured in the previous section as $0.74_{-0.10}^{+0.12}$ (stat.) \pm 0.04 (syst.), and disfavors null oscillation with a $96.0 \%$ confidence limit. The error coverage limits of this ratio are discussed in B.1 along with the coverage limits of the null-oscillation hypothesis. In this section the double-ratio is analyzed in the context of neutrino oscillation.

Using the 2-D oscillation-parameter grid described in Section 8.1 the double ratio is measured at each grid point by comparing the expected number of tracks and showers to the expected number of tracks and showers for null oscillation. In Appendix B.1, the procedure was described by which the null oscillation hypothesis is determined to disfavor the measured double ratio. Each point on the oscillation grid is treated as an oscillation hypothesis, and the same procedure can determine with what confidence the measured double ratio disfavors each oscillation grid point. At each point on the grid, $1 \times 10^{6}$ experiments are generated. The value of each double-sided Neyman confidence limit can be seen in the oscillation grid in Figure 8.2 a with the confidence on the Z-axis, and in Figure 8.2 b with $68 \%$ and $90 \%$ contours shown.
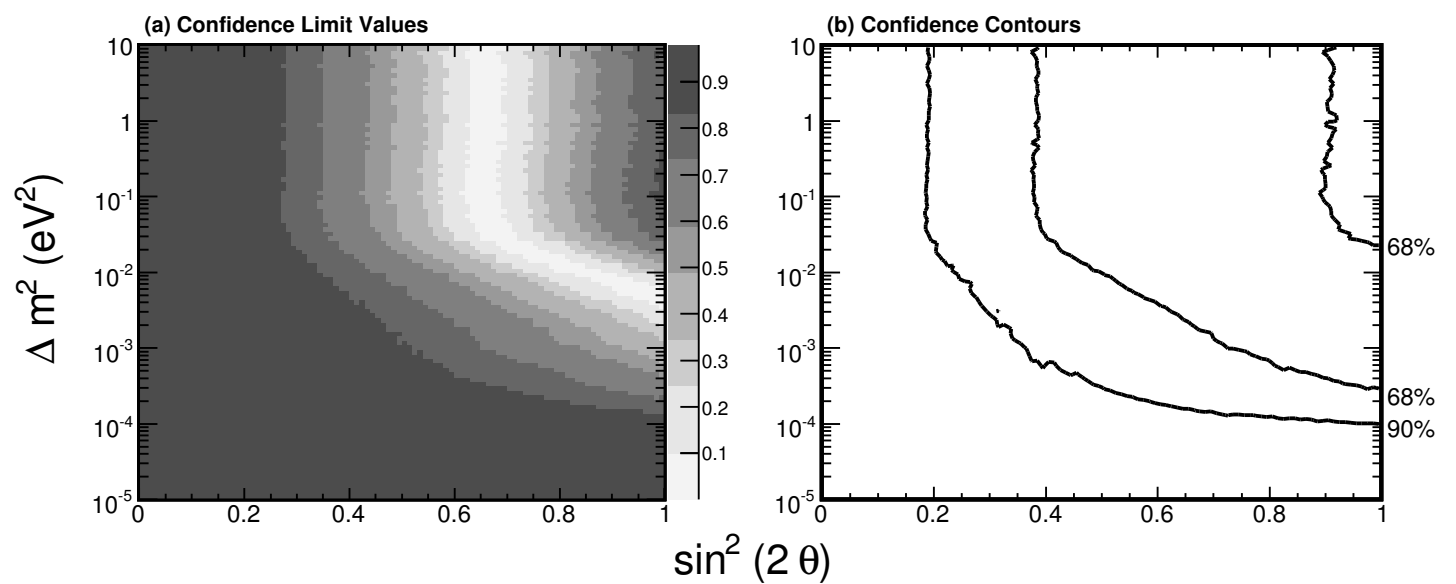

Figure 8.2: Frequentist Confidence limits for oscillation parameters displayed for (a) All Values and (b) Contours for relevant confidence limits relative to neutrino oscillation.

The flux scale factor was measured in Section 7.7 .2 with the observed and expected number of showering events using Equation 7.17. To enhance this measurement with the frequentist oscillation analysis, the number of expected showers can also be calculated 
as a result of the re-weighting procedure.
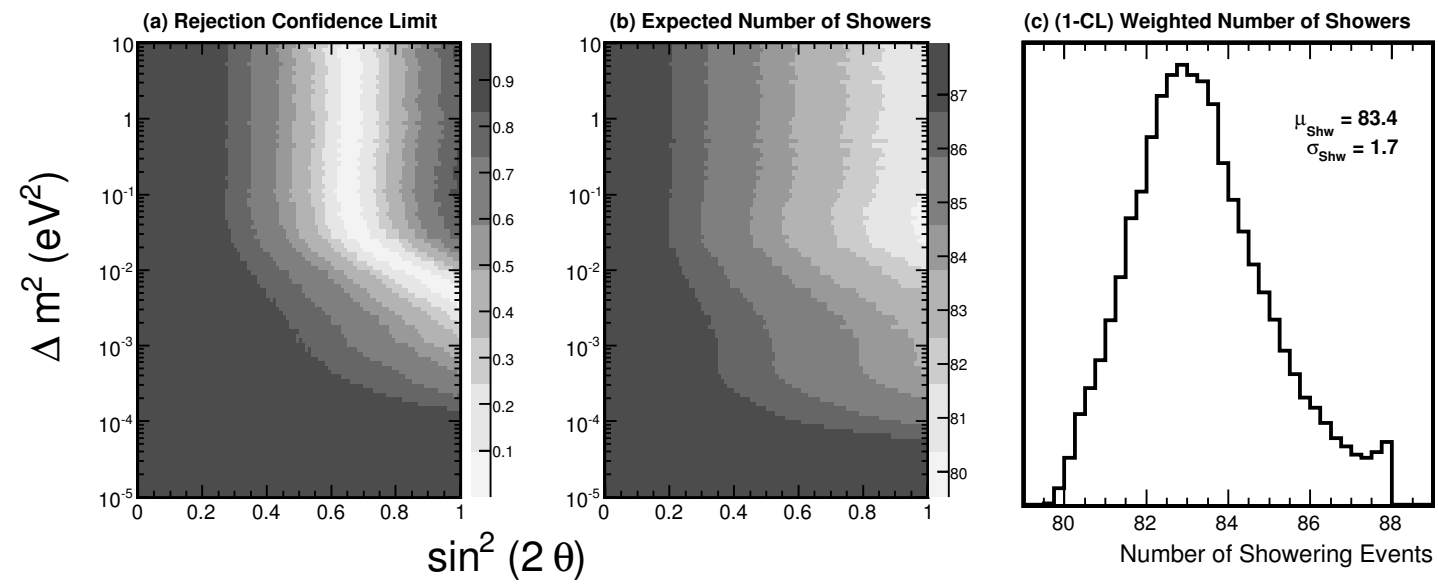

Figure 8.3: Number of showering event expressed as (b) a function of oscillation parameters and (c) weighted by (1-CL) from the oscillation analysis in (a).

Figure 8.3. b shows the expected number of showering events, as a result of oscillation. The confidence limit on the oscillation grid in Figure 8.3 a has been combined with the expected number of showers from Figure 8.3. b to weight the number of showers at each grid point by $1-C L$. The results is shown in Figure 8.3. c, where the mean number of showering events is $83.4 \pm 1.7$. The expected number of events had been 88.0 without accounting for oscillation, and this resulting in a scale factor of 1.01.

The new number of showering events is comprised of a number of neutrinos $\left(E_{\nu}=\right.$ 81.78), a number of cosmic muons $\left(E_{\mu}=3.81\right)$, and a number of neutrons $\left(E_{N}=2.36\right)$. The atmospheric scale factor can be measured based on this composition of showering neutrino interactions

The systematic errors described in Section 7.7 .1 have been explored, and are expressed in Table 8.1. The errors in this table are only for the Bartol flux model. The other two models discussed in Section 7.7.2 have run through a similar method to measure the flux scale, the results of which are:

$$
\begin{aligned}
S_{a t m}: \text { Bartol } & =1.07 \pm 0.12(\text { stat. }) \pm 0.09(\text { syst. }) \\
S_{a t m}: \text { Battistoni } & =1.22 \pm 0.13(\text { stat. }) \pm 0.09(\text { syst. }) \\
S_{a t m}: \text { Gheisha } & =0.96 \pm 0.11(\text { stat. }) \pm 0.07 \text { (syst. })
\end{aligned}
$$




\begin{tabular}{|c|c|c|}
\hline Systematic & $S_{\text {atm }}$ & $\Delta S_{a t m}(\%)$ \\
\hline 10 keV Cut-off & 1.02 & -4.69 \\
QE x-sect $\pm 10 \%$ & 1.05 & $\mp 2.87$ \\
& 1.10 & \\
NC x-sect $\pm 25 \%$ & 1.04 & $\mp 5.40$ \\
& 1.14 & \\
Neutron Flux $\pm 20 \%$ & 1.07 & $\mp 0.670$ \\
Oscillation Uncertainty & 1.07 & 2.12 \\
\hline Cumulative Error & & 8.02 \\
\hline
\end{tabular}

Table 8.1: Systematic errors for the measurement of the flux scale factor measured with the rate of showering atmospheric-neutrino interactions modified by oscillation as measured with a frequentist fit of the double ratio. The cumulative error is found by adding all error sources in quadrature.

The flux scales measured above are comparable to those in Section 7.7 .2 Accounting for oscillation has raised the flux scale by an amount that is barely within statistical error, and with a larger data set the oscillation enhanced normalization would start to become important. The systematic errors on this method are a bit larger than those in Section 7.7.2 due in part to the contribution of the oscillation uncertainty in the expected number of showering events. The alternative oscillation analysis in next section measures the flux scale simultaneously with the two oscillation parameters, and reduces the systematic from the oscillation uncertainty.

\subsection{Likelihood Oscillation Analysis}

The analytic measurement of the scale factor in Section 8.2 relies on the oscillation analysis combined with the method in Section 7.7.2. In this section, the scale factor is obtained In this section, the atmospheric-neutrino model scale factor is obtained by combining these two stages of analysis into a single stage. A maximum likelihood fit is applied to the number of showers and number of tracks for a best-fit set of oscillation parameters and a nuisance parameter for a scale factor is applied to the neutrino flux.

The "maximum-likelihood method" is, in principle, the process of maximizing the likelihood that an expected quantity will fluctuate to an observed quantity. Specifically, two distributions (one expected and one observed) are compared bin by bin, where the 
overall likelihood would be the product of the individual bin likelihoods. The content of a bin is a number of events, and is thus a Poisson distributed quantity. Therefore, Equation 8.4 expresses the likelihood that an expected distribution $\left(E_{i}\right)$ will fluctuate into an observed distribution $\left(O_{i}\right)$.

$$
\mathcal{L}=\prod_{i}\left(\frac{E_{i}^{O_{i}} e^{-E_{i}}}{O !}\right)
$$

The application of Equation 8.4 is not practically convenient, so the standard approach to calculate a maximum likelihood is to minimize the negative-log likelihood. The minimum of the negative-log likelihood will occur at the same fit parameters as the maximum of the likelihood. The negative-log likelihood, when applied to a Gaussian probability distribution (8.5), is expressed in Equation 8.6. The confidence regions in a one dimensional Gaussian distribution are mapped out as the $\chi_{\text {minimum }}^{2}+\Delta$ intervals. Equation 8.7 shows how this analogous relationship is drawn between the $\chi^{2}$ and $\Delta[-2 \ln (\mathcal{L})]$.

$$
\begin{aligned}
\mathcal{L}_{\text {Gaussian }}(x: \mu, \sigma) & =\frac{1}{\sigma \sqrt{2 \pi}} e^{(x-\mu)^{2} / 2 \sigma^{2}} \\
-\ln \left(\mathcal{L}_{\text {Gaussian }}(x: \mu, \sigma)\right) & =\frac{(x-\mu)^{2}}{2 \sigma^{2}}-\ln (\sigma \sqrt{2 \pi}) \\
\chi^{2}=\frac{(x-\mu)^{2}}{2 \sigma^{2}} & =\Delta\left(-2 \ln \left(\mathcal{L}_{\text {Gaussian }}\right)\right)
\end{aligned}
$$

Equation 8.8 shows the form of negative-log likelihood when Poisson distributed bins are used. The additional sum term summed over $j$ applies Gaussian "penalty terms" for "nuisance parameters". A nuisance parameter $\left(S_{j}\right)$ is allowed to vary in a fit, but has theoretically predetermined knowledge regarding the optimal value $\left(S_{j}^{0}\right)$ and uncertainty $\left(\sigma_{j}\right)$ of the nuisance parameter. Penalty terms allow for the correction of systematic errors in a fit, and presume that a nuisance parameter is Gaussian distributed.

$$
\Delta[-2 \ln (\mathcal{L})]=\sum_{i} 2\left(E_{i}-O_{i} \ln \frac{O_{i}}{E_{i}}\right)+\sum_{j} \frac{\left(S_{j}-S_{j}^{0}\right)^{2}}{\sigma_{j}^{2}}
$$

There are two extreme cases of the uncertainty on the penalty term. The first is where $\sigma \rightarrow 0$, which will make the penalty term infinite if $S_{j} \neq S_{j}^{0}$. This first case 
constrains the nuisance parameter to its optimal value. The second case occurs when $\sigma \rightarrow \infty$, which will make the penalty term vanish for all values of $S_{a t m}$. This second case removes the penalty that would have been applied for the nuisance parameter, and treats it like a unconstrained fit parameter.

The fit of interest presumes two bins, one for the shower count and one for the track count. These bins are fitted over the two oscillation parameters $\left(\sin ^{2}(2 \theta), \Delta m^{2}\right)$, and the flux scale factor $\left(S_{a t m}\right)$. There are three free parameters, and only two bins, which results in an under-constrained fit. If the flux scale factor is treated like a nuisance parameter to be Gaussian distributed about an optimal value of 1.0 with a given uncertainty, then the fit becomes constrained.

In either the constrained or under-constrained fits, the best neutrino scale factor can be solved for analytically. The negative-log likelihood function is minimized to find best fit parameters, and a best neutrino flux scale parameter will exist for each oscillation parameter pair. Finding each of these best flux scales numerically is a CPU intensive process. The best flux scale is solved analytically instead by finding the point where the derivative of the negative-log likelihood with respect to the flux scale approaches zero. This method is outlined more completely in Appendix Section B.2

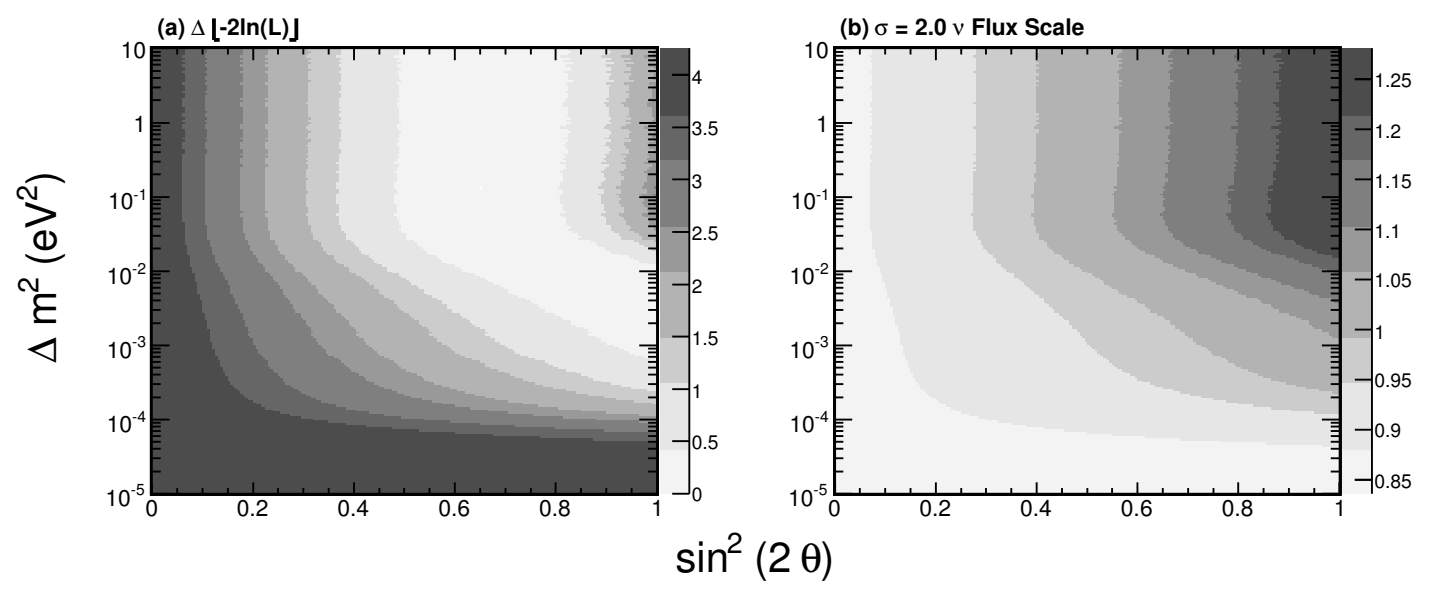

Figure 8.4: Likelihood fit of shower and track counts with the scale factor weakly penalized $(\sigma=2.0)$.

In order to measure the flux scale factor, the likelihood fit must be made either 
without the flux-scale-penalty factor, or with a large uncertainty on the flux-scalepenalty factor. The values displayed in Figure 8.4 use a penalty factor with $\sigma=2.0$, such that the scale factor is allowed to vary with a small enough constraint to account for the neutrino deficit. Figure 8.4 a shows a $200 \times 200$ grid of the oscillation parameters with the value of the $\Delta[-2 \ln (\mathcal{L})]$ at each parameter. The analytically solved flux scale factor on the same grid is shown in Figure $8.4 \mathrm{~b}$.

To measure the flux scale in the scheme shown in Figure 8.4 the optimal scale factor that is solved for each grid point corresponds to a $\Delta[-2 \ln (\mathcal{L})]$ at the same grid point. In Figure 8.5 a, each point displays the minimum value of $\Delta[-2 \ln (\mathcal{L})]$ on the Y-axis for a flux scale on the X-axis. The points that are near $\Delta[-2 \ln (\mathcal{L})]=1.0$ on both side of the parabolic shape are fit to straight lines, and the intercepts these lines with $\Delta[-2 \ln (\mathcal{L})]=1.0$ are used to find the errors on the flux scale.
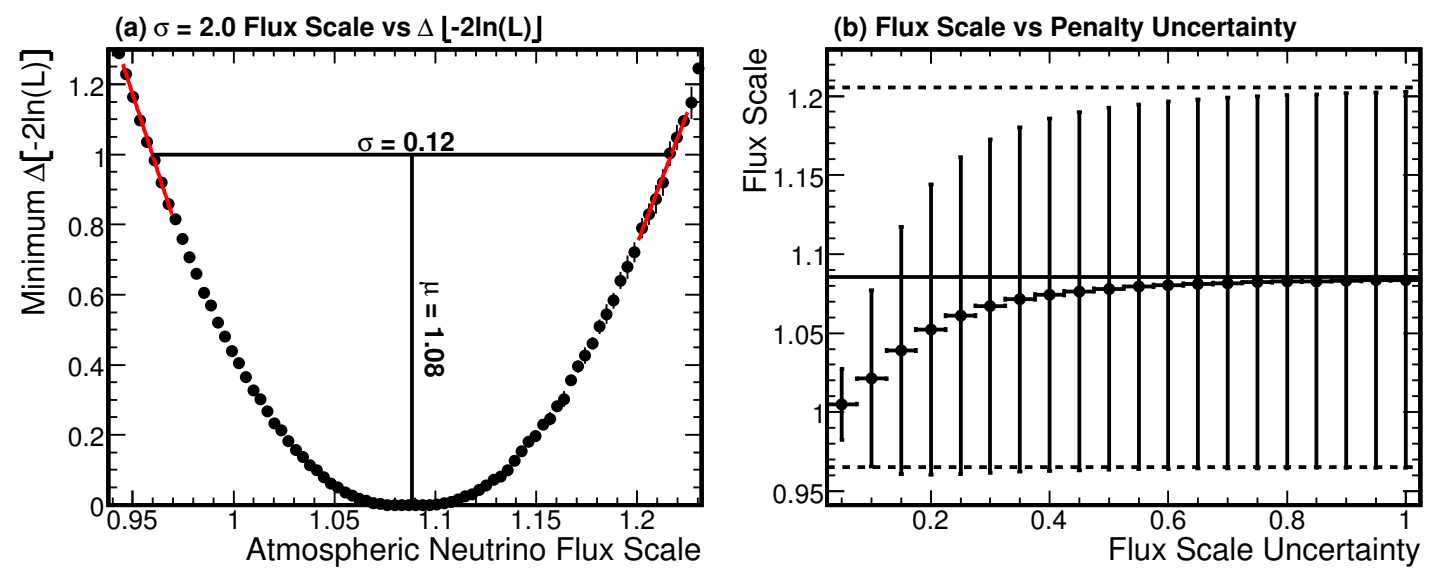

Figure 8.5: The average $\Delta[-2 \ln (\mathcal{L})]$ is shown for a scale factor as a result of extending the values in Figures $8.4 \mathrm{a}$ and $8.4 \mathrm{~b}$.

If the uncertainty of the penalty is reduced, such that the flux scale in no longer "weakly penalized", the solution to the flux scale factor converges towards 1.0, and the error will approach 0.0. Figure 8.5. b shows the result of decreasing the penalty uncertainty from 1.0 to 0.05 . The flux model [2] quotes a flux uncertainty of $20 \%$, which, in Figure 8.5. b, corresponds to $S_{a t m}=1.04 \pm 0.09$.

The scale factor $S_{a t m}=1.09 \pm 0.12$ measured by the maximum likelihood fit method agrees with $S_{a t m}=1.07 \pm 0.12$, measured in Section 8.2. The systematic errors expressed 
in Table 8.1 for the method used in Section 8.2 are carried over to the likelihood method, and expressed in Table 8.2 The systematic error from the oscillation uncertainty has been included in the fit, and is no longer needed in the systematics.

\begin{tabular}{|c|c|c|}
\hline Systematic & $S_{a t m}$ & $\Delta S_{a t m}(\%)$ \\
\hline 10 keV Cut-off & 1.02 & -4.99 \\
QE x-sect $\pm 10 \%$ & 1.06 & $\mp 2.83$ \\
& 1.11 & \\
NC x-sect $\pm 25 \%$ & 1.03 & $\mp 5.41$ \\
& 1.14 & \\
Neutron Flux $\pm 20 \%$ & 1.07 & $\mp 0.665$ \\
\hline Cumulative Error & 1.09 & 7.84 \\
\hline
\end{tabular}

Table 8.2: Systematic errors for the measurement of the flux scale factor measured with the rate of showering atmospheric-neutrino interactions. The cumulative error is found by adding all error sources in quadrature.

The removal of the oscillation uncertainty reduces the systematic error by a small amount. The flux scale factor now is $S_{a t m}=1.07 \pm 0.12$ (stat.) \pm 0.08 (syst.), which has the same systematic error within a significant digit of the error measured in Section 8.2 . The pattern of the individual systematic errors in Table 8.2 follows the pattern of errors in Table 8.1 The scale factors for all three relevant models are now:

$$
\begin{aligned}
S_{\text {atm }}: \text { Bartol } & =1.08 \pm 0.12(\text { stat. }) \pm 0.08(\text { syst. }) \\
S_{a t m}: \text { Battistoni } & =1.23 \pm 0.13(\text { stat. }) \pm 0.09(\text { syst. }) \\
S_{\text {atm }}: \text { Gheisha } & =0.98 \pm 0.12(\text { stat. }) \pm 0.07 \text { (syst. })
\end{aligned}
$$

The flux scale factors in Equation 8.9 compare favorably to those in Equation 8.3, and both oscillation analyses have produced a consistent measurement for the neutrino flux scale. These two methods produce likelihood contours for oscillation parameters that should also be comparable. To lessen the difficulty of comparing two dimensional contours, one dimensional slices can be made in the oscillation grid, and the oscillation parameters in these slices are compared to each other.

The first comparison in Figure 8.6 takes the slice in the oscillation grid at the 


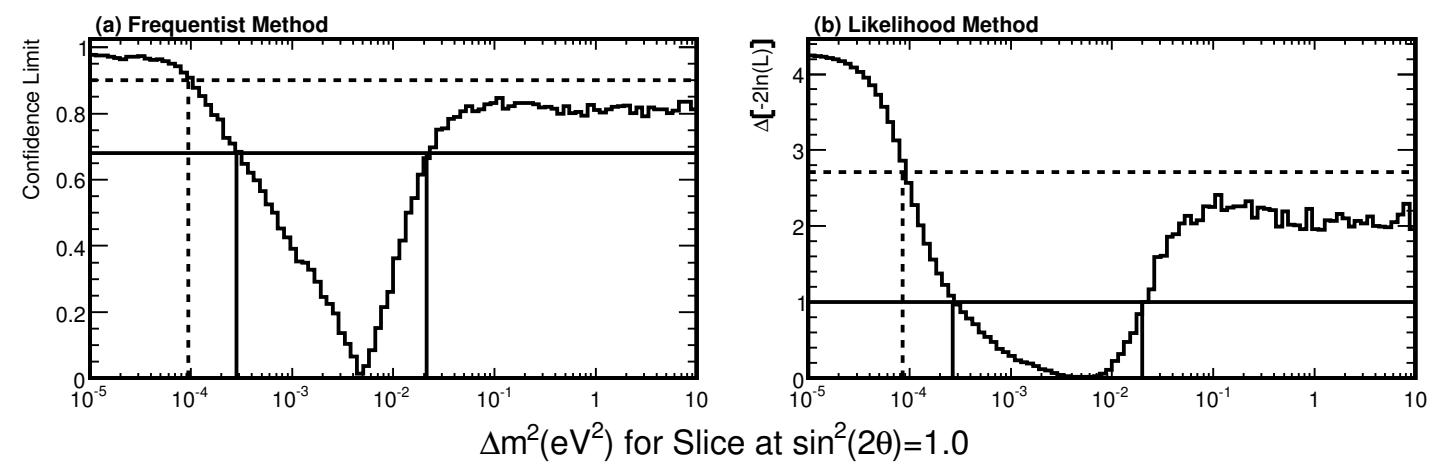

Figure 8.6: Slices at maximal mixing in (a) frequentist and (b) likelihood fit to track and shower count for oscillation spaces.

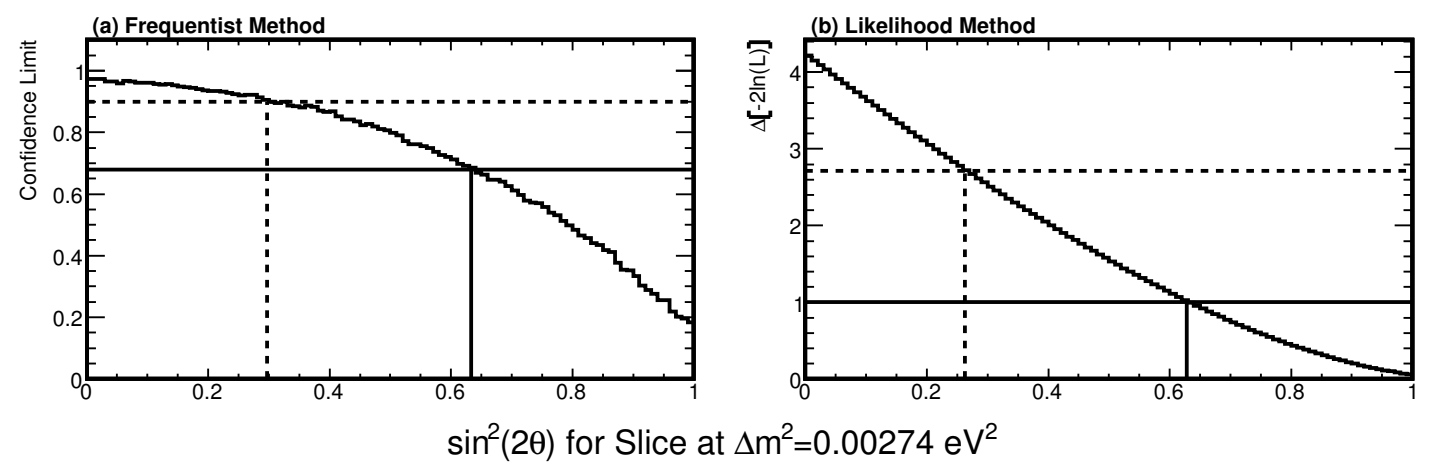

Figure 8.7: Slices at the best fit value of $\Delta m^{2}=0.00274 \mathrm{eV}^{2}$ obtained by the MINOS beam experiment in (a) frequentist fit and (b) likelihood track and shower count fit oscillation spaces. 
maximal mixing solution $\left(\sin ^{2}(2 \theta)=1.0\right)$. Maximal mixing is favored by many muonneutrino disappearance experiments [1, 4, 95]. The shape of the Frequentist (Figure 8.6.a) and Likelihood (Figure 8.6.b) slices differ at the minimum values significantly. The difference is understood to be a combination of two effects: (1) the first method fits to a single value (double-ratio) while the second method fits to two values (track and shower counts), (2) the first method displays a confidence limit, while the second displays the Gaussian $c h i^{2}$ equivalent value of $\Delta[-2 \ln (\mathcal{L})]$.

The second comparison in Figure 8.7 takes slices in the oscillation grid at the solution for $d m s q=0.00274 \mathrm{eV}^{2}$ favored by the MINOS long-baseline muon-neutrino disappearance experiment [95. The shape differs between the Frequentist (Figure 8.7]a) and Likelihood (Figure 8.7.b) methods for the same reasons the shapes differed for the maximal-mixing slices in Figure 8.6.

In all of the slice figures, horizontal lines are drawn to represent the $68 \%$ (solid line) and $90 \%$ (dashed line) confidence limits. Vertical lines are also drawn at the values in $\Delta m^{2}$ and $\sin ^{2}(2 \theta)$ that fall on the $68 \%$ and $90 \%$ confidence limits with the corresponding line styles. The confidence limits in any Frequentist fit are determined directly in the process of the fit. For the Likelihood fit, the $68 \%(\Delta[-2 \ln (\mathcal{L})]=1.0)$ and $90 \%(\Delta[-2 \ln (\mathcal{L})]=2.71)$ confidence limits are determined by integrating a single parameter Gaussian probability distribution function. Table 8.3 compares the values resulting from the two methods at the best fit and for $68 \%$ and $90 \%$ confidence limits.

\begin{tabular}{|c|c|c|c|}
\hline \multicolumn{2}{|c|}{ Oscillation Grid Slice } & Frequentist Fit & Likelihood Fit \\
\hline $\mathbf{\Delta m}^{\mathbf{2}}\left(\mathbf{e V}^{\mathbf{2}}\right)$ for & Best $\Delta m^{2}$ & $4.08 \times 10^{-3}$ & $4.08 \times 10^{-3}$ \\
$\sin ^{\mathbf{2}}(\mathbf{2} \theta)=\mathbf{1 . 0}$ & $68 \% C L$ & $2.43 \times 10^{-4}$ & $2.28 \times 10^{-4}$ \\
& $90 \% C L$ & $2.02 \times 10^{-2}$ & $1.78 \times 10^{-2}$ \\
& Best $\sin ^{2}(2 \theta)$ & $1.84 \times 10^{-5}$ & $7.62 \times 10^{-5}$ \\
\hline $\sin ^{2}(\mathbf{2} \theta)$ for & $68 \% C L$ & 0.611 & 1.0 \\
$\mathbf{\Delta m}^{\mathbf{2}}=\mathbf{0 . 0 0 2 7 4} \mathbf{e V}^{\mathbf{2}}$ & $90 \% C L$ & 0.287 & 0.592 \\
& & 0.220 \\
\hline
\end{tabular}

Table 8.3: Oscillation parameters compared between Frequentist and Likelihood fitting methods. 


\subsection{Projected Sensitivity}

Appendix B.1 describes in detail the measurement of the double ratio error statistical error. The exploration of the systematic errors of the double ratio from Section 7.7 .1 yielded that the average statistical error (0.105) dominates the average systematic error (0.06) for the double ratio. As more data is collected over the course of the experiment this statistical error will decrease in a predictable manner, but the systematic error will be reduced by unpredictable steps of increased experimental understanding.

The fiducial exposure of the data sample used in this thesis is $4.526 \mathrm{kty}$, for cumulative 418.5 live days of data collection from August 2003 to February 2005. The exposure will increase with the future of the experiment as more data is collected. The run plan for the MINOS experiment is to continue taking data until 2010, which would add 5 years of data. If the far detector is on for $98 \%$ of that time, then the exposure for the 3.94 kton fiducial volume will be $23.83 \mathrm{kty}$ by the end of the experiment.

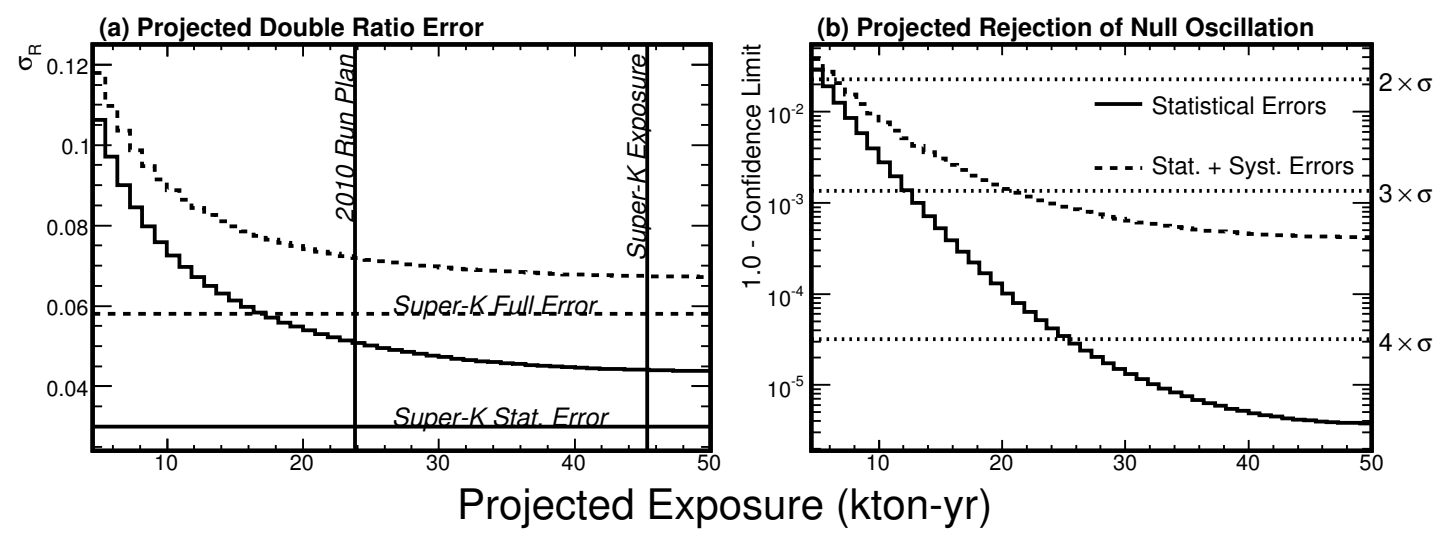

Figure 8.8: Projected sensitivity of the double ratio measurement, (a) error of the double ratio, and (b) rejection of the null oscillation hypothesis.

Figure 8.8 a shows how both the statistical error and statistical + systematic error (added in quadrature) will decrease for increased exposure out to the projected 2010 run plan. The Super-K measurement of the double ratio was $0.67 \pm 0.03$ (stat.) \pm 0.05 (syst.) for a 45.34 kty fiducial exposure [32]. The 3.94 kton fiducial mass of the MINOS far detector can not compete with the $22.5 \mathrm{kton}$ fiducial mass of the Super$\mathrm{K}$ detector. However, the performance for selecting atmospheric neutrino interactions can be compared with between the MINOS and Super-K experiment by comparing the 
projected error at an equivalent exposure.

Figure 8.8 a shows how well the projected double ratio will reject the null oscillation hypothesis. The rejection confidence limits are single-sided limits, but for added perspective there are lines for the equivalent Gaussian double-sided rejection of the null-oscillation hypothesis at 2,3 , and $4 \times \sigma$. 


\section{Chapter 9}

\section{Conclusion and Discussion}

The electron neutrino component of atmospheric neutrinos has been used to analyze the atmospheric neutrino flux, and combined with the muon neutrino component has also been used to analyze the oscillation of atmospheric neutrinos. The three analytic goals of this thesis were as follows.

1. Find the atmospheric neutrino double ratio (observed over expected) of the muon neutrino interaction rate over the electron neutrino interaction rate.

2. Evaluate atmospheric neutrino oscillation based on the observed interaction rates of electron and muon neutrinos.

3. Measure the atmospheric neutrino flux based on the electron neutrino interaction rate.

The atmospheric neutrino double ratio required the redefinition to the ratio of tracklike over showering event rate, for practical purposes. In order to measure these event rates, track-like and showering events culled from the data with event selection processes that were optimized with the use of simulated signal and backgrounds sets. The cosmicray veto shield both improved the background rejection of the event selection and offered an estimate of background contamination of the selected events.

The contained vertex track-like and showering events rates were measured for a data set of 418.5 live days, or a fiducial exposure of 4.18 kty. The double ratio was measured with these observed event rates combined with the expected event rates from 
the simulated sets. The statistical error on the double ratio was measured precisely with Monte Carlo methods, and the systematic error was measured by applying systematic variations to the simulated set.

The double ratio has been used to find the confidence intervals in the 2-neutrino oscillation-parameter space with a Neyman frequentist fit. These confidence intervals were then used to find the oscillation-adjusted expected showering neutrino event rate. This adjusted rate was used to measure the atmospheric neutrino flux. Systematic variances were also applied to the measurement of the neutrino flux to estimate the systematic error of the neutrino flux measurement.

To confirm the results from the frequentist fit of the double ratio, a second fit was performed. The two-neutrino oscillation parameters and the atmospheric neutrino flux were all fit to the showering and track-like event rates. The resulting neutrino flux from this measurement was equivalent to that from the Neyman frequentist fit.

The analysis of atmospheric electron neutrinos has been used to describe oscillation and measure the atmospheric neutrino flux. Both of these measurements are limited by statistical error, and would benefit from increased data. The systematic errors are close to the statistical errors though, and if data is to be added to improve the statistics, the systematics will have to be dealt with as well. The measurements made in this thesis have a promising future, and much work yet to be done. 


\section{Appendix A}

\section{Selected Events}

The data set which I have analyzed for neutrinos runs from 2003/08/01 to 2005/02/01, for 418.5 live days, and spans run numbers 18143 to 29325 . In this set, I have selected 89 showers, 72 fully-contained tracks, 25 partially-contained downward tracks, and 14 partially-contained tracks. These events are outlined below with parameters relevant to the oscillation analysis. For a more complete outline of the events, including parameters relevant to the full selection process and event displays, visit

http://webusers.physics.umn.edu/〜bspeak/events/.

\section{A.1 Selected Showers}

Table A.1: Selected Fully Contained Showers

\begin{tabular}{|c|c|c|c|c|c|c|}
\hline$\#$ & Run & Snarl & Date & ShwE $(\mathrm{GeV})$ & $\operatorname{VtxR}(\mathrm{m})$ & Plane Range \\
\hline 1 & 18884 & 72289 & $2003 / 08 / 31$ & 3.5 & 0.879 & $392-401$ \\
2 & 18964 & 82342 & $2003 / 09 / 02$ & 3 & 1.39 & $11-33$ \\
3 & 19241 & 46997 & $2003 / 09 / 11$ & 0.52 & 2.08 & $114-118$ \\
4 & 19316 & 1858 & $2003 / 09 / 15$ & 1.4 & 1.95 & $80-86$ \\
5 & 19896 & 37336 & $2003 / 10 / 02$ & 2.1 & 1.79 & $302-306$ \\
6 & 19961 & 62410 & $2003 / 10 / 05$ & 2.6 & 1.46 & $426-450$ \\
7 & 20456 & 56981 & $2003 / 10 / 17$ & 0.61 & 2.68 & $259-266$ \\
\hline \multicolumn{5}{|l|}{ Continued on next page } \\
\hline
\end{tabular}


Table A.1 - continued from previous page

\begin{tabular}{|c|c|c|c|c|c|c|}
\hline$\#$ & Run & Snarl & Date & ShwE $(\mathrm{GeV})$ & VtxR $(\mathrm{m})$ & Plane Range \\
\hline 8 & 20509 & 66401 & $2003 / 10 / 19$ & 2.5 & 1.54 & $345-360$ \\
9 & 21067 & 50599 & $2003 / 11 / 05$ & 0.79 & 0.73 & $403-432$ \\
10 & 21234 & 24172 & $2003 / 11 / 10$ & 0.76 & 3.49 & $304-315$ \\
11 & 21263 & 34713 & $2003 / 11 / 11$ & 2.2 & 1.59 & $418-427$ \\
12 & 21283 & 39583 & $2003 / 11 / 12$ & 1.4 & 1.48 & $288-298$ \\
13 & 21406 & 18579 & $2003 / 11 / 17$ & 3.2 & 0.903 & $450-462$ \\
14 & 21768 & 4754 & $2003 / 12 / 03$ & 1.6 & 1.09 & $378-391$ \\
15 & 21849 & 20088 & $2003 / 12 / 07$ & 1.7 & 2.64 & $404-420$ \\
16 & 21857 & 64634 & $2003 / 12 / 08$ & 1.2 & 1.2 & $99-106$ \\
17 & 21906 & 119788 & $2003 / 12 / 12$ & 1.2 & 1.19 & $35-42$ \\
18 & 21927 & 91817 & $2003 / 12 / 13$ & 2.9 & 1.48 & $77-85$ \\
19 & 22150 & 188512 & $2003 / 12 / 23$ & 1.8 & 1.1 & $438-445$ \\
20 & 22180 & 208482 & $2003 / 12 / 26$ & 3.1 & 3.24 & $262-276$ \\
21 & 22260 & 23994 & $2004 / 01 / 03$ & 1.2 & 0.957 & $170-174$ \\
22 & 22260 & 195827 & $2004 / 01 / 03$ & 5.5 & 1.29 & $317-337$ \\
23 & 22294 & 150907 & $2004 / 01 / 06$ & 11 & 2.05 & $35-59$ \\
24 & 22461 & 39901 & $2004 / 01 / 17$ & 1.6 & 2.07 & $393-406$ \\
25 & 22678 & 53855 & $2004 / 02 / 03$ & 0.62 & 2.36 & $82-88$ \\
26 & 22705 & 128426 & $2004 / 02 / 06$ & 1 & 1.1 & $454-467$ \\
27 & 22734 & 141714 & $2004 / 02 / 09$ & 0.66 & 1.19 & $464-469$ \\
28 & 22740 & 103251 & $2004 / 02 / 09$ & 0.87 & 2.62 & $415-431$ \\
29 & 22989 & 51048 & $2004 / 02 / 29$ & 1.8 & 2.91 & $50-67$ \\
30 & 23004 & 25675 & $2004 / 03 / 02$ & 6.3 & 1.64 & $175-190$ \\
31 & 23285 & 69849 & $2004 / 03 / 14$ & 0.52 & 1.28 & $431-436$ \\
32 & 23297 & 48900 & $2004 / 03 / 15$ & 0.51 & 2.69 & $295-299$ \\
33 & 23715 & 73690 & $2004 / 03 / 21$ & 1.7 & 0.716 & $134-141$ \\
34 & 23952 & 29643 & $2004 / 03 / 24$ & 5.3 & 0.906 & $405-416$ \\
35 & 24070 & 5664 & $2004 / 03 / 30$ & 0.82 & 0.725 & $14-30$ \\
36 & 24829 & 44267 & $2004 / 04 / 09$ & 8.3 & 1.23 & $63-81$ \\
\hline & & & & & Continued on next page \\
\hline
\end{tabular}


Table A.1 - continued from previous page

\begin{tabular}{|c|c|c|c|c|c|c|}
\hline$\#$ & Run & Snarl & Date & ShwE $(\mathrm{GeV})$ & VtxR(m) & Plane Range \\
\hline 37 & 24859 & 70193 & $2004 / 04 / 12$ & 1.4 & 2.25 & $44-54$ \\
38 & 24871 & 1546 & $2004 / 04 / 12$ & 0.92 & 3.07 & $66-75$ \\
39 & 24907 & 19211 & $2004 / 04 / 15$ & 4.8 & 0.583 & $362-380$ \\
40 & 24985 & 95692 & $2004 / 04 / 20$ & 0.67 & 0.685 & $101-121$ \\
41 & 25115 & 14292 & $2004 / 04 / 30$ & 2 & 0.607 & $277-290$ \\
42 & 25151 & 55889 & $2004 / 05 / 04$ & 0.5 & 1.51 & $18-22$ \\
43 & 25283 & 8536 & $2004 / 05 / 11$ & 1.7 & 0.863 & $359-372$ \\
44 & 25304 & 72062 & $2004 / 05 / 13$ & 1.7 & 0.672 & $369-377$ \\
45 & 25322 & 11164 & $2004 / 05 / 14$ & 1.5 & 2.44 & $364-376$ \\
46 & 25493 & 141108 & $2004 / 05 / 27$ & 2 & 0.629 & $349-355$ \\
47 & 25337 & 82609 & $2004 / 05 / 16$ & 1.2 & 0.807 & $259-264$ \\
48 & 25355 & 119017 & $2004 / 05 / 18$ & 4.3 & 1.17 & $129-141$ \\
49 & 25592 & 26856 & $2004 / 06 / 06$ & 5.8 & 2.31 & $70-80$ \\
50 & 25610 & 47904 & $2004 / 06 / 07$ & 1.7 & 0.938 & $337-347$ \\
51 & 25689 & 96159 & $2004 / 06 / 15$ & 1.6 & 1.6 & $388-395$ \\
52 & 26169 & 1669 & $2004 / 07 / 21$ & 1.2 & 1.61 & $112-121$ \\
53 & 26277 & 16070 & $2004 / 07 / 22$ & 0.4 & 2.41 & $301-309$ \\
54 & 26320 & 25347 & $2004 / 07 / 27$ & 1.5 & 1.09 & $114-121$ \\
55 & 26362 & 9853 & $2004 / 07 / 30$ & 2.8 & 1.41 & $397-405$ \\
56 & 26537 & 46199 & $2004 / 08 / 10$ & 1.1 & 3.18 & $72-81$ \\
57 & 26983 & 62805 & $2004 / 08 / 30$ & 1.8 & 1.62 & $133-137$ \\
58 & 27033 & 50421 & $2004 / 09 / 03$ & 3.3 & 0.547 & $220-232$ \\
59 & 27094 & 104440 & $2004 / 09 / 08$ & 2.2 & 1.21 & $333-343$ \\
60 & 27118 & 16220 & $2004 / 09 / 09$ & 0.95 & 2.04 & $287-300$ \\
61 & 27139 & 65249 & $2004 / 09 / 11$ & 1.2 & 0.611 & $430-436$ \\
62 & 27154 & 70014 & $2004 / 09 / 13$ & 2.1 & 2.03 & $424-440$ \\
63 & 27190 & 93280 & $2004 / 09 / 16$ & 4 & 3.34 & $125-138$ \\
64 & 27292 & 181 & $2004 / 09 / 23$ & 2.2 & 1.3 & $352-369$ \\
65 & 27319 & 90167 & $2004 / 09 / 25$ & 3.4 & 1.15 & $113-124$ \\
\hline & & & & & Continued on next page \\
\end{tabular}


Table A.1 - continued from previous page

\begin{tabular}{|c|c|c|c|c|c|c|}
\hline$\#$ & Run & Snarl & Date & ShwE $(\mathrm{GeV})$ & VtxR $(\mathrm{m})$ & Plane Range \\
\hline 66 & 27482 & 40833 & $2004 / 10 / 03$ & 0.72 & 3.4 & $263-271$ \\
67 & 27491 & 108894 & $2004 / 10 / 04$ & 1 & 0.748 & $342-348$ \\
68 & 27523 & 83334 & $2004 / 10 / 07$ & 1.7 & 0.926 & $98-107$ \\
69 & 27542 & 54412 & $2004 / 10 / 08$ & 0.51 & 1.47 & $390-394$ \\
70 & 27554 & 4877 & $2004 / 10 / 08$ & 1.7 & 2.12 & $199-206$ \\
71 & 27572 & 10154 & $2004 / 10 / 10$ & 2.4 & 0.976 & $398-417$ \\
72 & 27636 & 35095 & $2004 / 10 / 15$ & 4.6 & 1.43 & $68-83$ \\
73 & 27654 & 26399 & $2004 / 10 / 17$ & 0.62 & 2.33 & $423-432$ \\
74 & 27675 & 9248 & $2004 / 10 / 20$ & 0.58 & 1.48 & $150-154$ \\
75 & 27737 & 78933 & $2004 / 10 / 26$ & 2.2 & 2.15 & $299-306$ \\
76 & 27796 & 6882 & $2004 / 11 / 01$ & 2 & 0.591 & $214-229$ \\
77 & 28000 & 95965 & $2004 / 11 / 19$ & 0.7 & 0.944 & $468-474$ \\
78 & 28220 & 5608 & $2004 / 12 / 01$ & 2.1 & 3.08 & $189-209$ \\
79 & 28232 & 45740 & $2004 / 12 / 03$ & 2.7 & 1.43 & $429-434$ \\
80 & 28303 & 40981 & $2004 / 12 / 09$ & 1.4 & 1.92 & $144-152$ \\
81 & 28330 & 76058 & $2004 / 12 / 11$ & 3.7 & 1.6 & $450-458$ \\
82 & 28410 & 22364 & $2004 / 12 / 18$ & 0.98 & 3.12 & $320-333$ \\
83 & 28603 & 66129 & $2005 / 01 / 07$ & 4.6 & 1.46 & $334-344$ \\
84 & 28618 & 143762 & $2005 / 01 / 09$ & 3.4 & 0.844 & $370-383$ \\
85 & 28986 & 100769 & $2005 / 01 / 20$ & 2 & 1.38 & $416-430$ \\
86 & 29005 & 5570 & $2005 / 01 / 22$ & 1.6 & 0.573 & $135-146$ \\
87 & 29052 & 49844 & $2005 / 01 / 27$ & 3.6 & 1.68 & $296-305$ \\
88 & 29070 & 55235 & $2005 / 01 / 29$ & 0.7 & 2.8 & $430-437$ \\
89 & 29079 & 42619 & $2005 / 01 / 30$ & 6.7 & 2.15 & $182-201$ \\
\hline
\end{tabular}

\section{A.2 Selected Tracks}

Tracks are divided into the three containment types, which I present in three separate tables 


\section{A.2.1 Fully Contained}

Table A.2: Selected Fully Contained Tracks

\begin{tabular}{|c|c|c|c|c|c|c|}
\hline$\#$ & Run & Snarl & Date & Cos $\theta_{\text {Zenith }}$ & VtxR(m) & Plane Range \\
\hline 1 & 18581 & 63807 & $2003 / 08 / 17$ & 0.54 & 2.18 & $29-44$ \\
2 & 18866 & 62010 & $2003 / 08 / 30$ & 0.372 & 2.08 & $398-431$ \\
3 & 20131 & 33435 & $2003 / 10 / 11$ & 0.618 & 1.08 & $297-313$ \\
4 & 20147 & 26192 & $2003 / 10 / 11$ & -0.15 & 0.706 & $110-117$ \\
5 & 20629 & 31711 & $2003 / 10 / 22$ & -0.478 & 2.21 & $165-208$ \\
6 & 20818 & 65032 & $2003 / 10 / 28$ & -0.555 & 0.826 & $452-470$ \\
7 & 20998 & 6938 & $2003 / 11 / 02$ & 0.00168 & 0.963 & $365-445$ \\
8 & 21737 & 63416 & $2003 / 12 / 02$ & -0.0533 & 1.64 & $362-386$ \\
9 & 21906 & 116585 & $2003 / 12 / 12$ & 0.677 & 1.14 & $190-198$ \\
10 & 22236 & 83171 & $2003 / 12 / 31$ & -0.314 & 1.74 & $406-418$ \\
11 & 22424 & 149574 & $2004 / 01 / 14$ & 0.394 & 1.8 & $369-378$ \\
12 & 22446 & 82423 & $2004 / 01 / 16$ & 0.265 & 3.03 & $281-374$ \\
13 & 22509 & 24855 & $2004 / 01 / 21$ & -0.388 & 3.17 & $173-192$ \\
14 & 22575 & 95688 & $2004 / 01 / 25$ & -0.299 & 1.37 & $266-273$ \\
15 & 22708 & 84455 & $2004 / 02 / 06$ & 0.496 & 2.85 & $339-370$ \\
16 & 22713 & 10258 & $2004 / 02 / 06$ & -0.01 & 1.65 & $192-200$ \\
17 & 22825 & 32924 & $2004 / 02 / 16$ & -0.384 & 1.44 & $57-64$ \\
18 & 22850 & 92736 & $2004 / 02 / 19$ & 0.616 & 1.14 & $301-315$ \\
19 & 22868 & 10353 & $2004 / 02 / 19$ & 0.249 & 1.16 & $43-57$ \\
20 & 22919 & 7988 & $2004 / 02 / 23$ & 0.0438 & 3.13 & $54-89$ \\
21 & 23285 & 59052 & $2004 / 03 / 14$ & -0.068 & 0.832 & $310-321$ \\
22 & 23297 & 43710 & $2004 / 03 / 15$ & -0.346 & 1.73 & $65-86$ \\
23 & 23604 & 95164 & $2004 / 03 / 18$ & 0.125 & 0.983 & $186-202$ \\
24 & 23700 & 125712 & $2004 / 03 / 20$ & -0.526 & 2.41 & $309-316$ \\
25 & 23943 & 85316 & $2004 / 03 / 23$ & 0.325 & 1.56 & $431-444$ \\
26 & 23998 & 9213 & $2004 / 03 / 28$ & -0.595 & 3.52 & $143-155$ \\
\hline & & & & & Continued on next page \\
\hline
\end{tabular}


Table A.2 - continued from previous page

\begin{tabular}{|c|c|c|c|c|c|c|}
\hline$\#$ & Run & Snarl & Date & Cos $\theta_{\text {Zenith }}$ & VtxR $(\mathrm{m})$ & Plane Range \\
\hline 27 & 24088 & 8719 & $2004 / 03 / 31$ & -0.297 & 1.88 & $335-352$ \\
28 & 24141 & 4689 & $2004 / 04 / 01$ & -0.781 & 1.5 & $237-262$ \\
29 & 24829 & 87042 & $2004 / 04 / 09$ & -0.244 & 0.941 & $89-110$ \\
30 & 24844 & 97277 & $2004 / 04 / 10$ & -0.692 & 2.31 & $268-302$ \\
31 & 24871 & 120533 & $2004 / 04 / 13$ & -0.704 & 2.44 & $109-127$ \\
32 & 24881 & 44348 & $2004 / 04 / 13$ & -0.562 & 1.89 & $373-391$ \\
33 & 24941 & 72237 & $2004 / 04 / 15$ & 0.628 & 1.11 & $325-341$ \\
34 & 24982 & 23731 & $2004 / 04 / 19$ & -0.193 & 2.75 & $327-396$ \\
35 & 24988 & 8655 & $2004 / 04 / 20$ & 0.323 & 1.83 & $434-466$ \\
36 & 25048 & 25582 & $2004 / 04 / 23$ & 0.849 & 1.25 & $423-436$ \\
37 & 25106 & 78421 & $2004 / 04 / 29$ & 0.515 & 1.33 & $90-99$ \\
38 & 25142 & 38534 & $2004 / 05 / 03$ & -0.0475 & 3.02 & $116-128$ \\
39 & 25154 & 96745 & $2004 / 05 / 04$ & -0.347 & 1.56 & $268-281$ \\
40 & 25283 & 18347 & $2004 / 05 / 11$ & 0.236 & 2.64 & $128-286$ \\
41 & 25313 & 5784 & $2004 / 05 / 13$ & -0.133 & 2.86 & $212-221$ \\
42 & 25505 & 57899 & $2004 / 05 / 28$ & -0.0739 & 2.24 & $402-410$ \\
43 & 25393 & 105572 & $2004 / 05 / 20$ & -0.447 & 0.718 & $24-32$ \\
44 & 25411 & 35592 & $2004 / 05 / 21$ & -0.617 & 1.29 & $10-39$ \\
45 & 25589 & 98333 & $2004 / 06 / 05$ & -0.14 & 1.41 & $327-339$ \\
46 & 25689 & 73688 & $2004 / 06 / 15$ & 0.464 & 0.817 & $38-55$ \\
47 & 25831 & 21795 & $2004 / 06 / 27$ & 0.545 & 0.767 & $394-401$ \\
48 & 25988 & 40460 & $2004 / 07 / 10$ & 0.86 & 1.04 & $54-81$ \\
49 & 25991 & 67835 & $2004 / 07 / 11$ & 0.172 & 0.772 & $348-367$ \\
50 & 25991 & 68957 & $2004 / 07 / 11$ & 0.51 & 1.95 & $283-367$ \\
51 & 26000 & 60501 & $2004 / 07 / 12$ & 0.335 & 1.28 & $133-144$ \\
52 & 26348 & 57497 & $2004 / 07 / 29$ & 0.788 & 0.972 & $294-303$ \\
53 & 27184 & 116702 & $2004 / 09 / 16$ & 0.204 & 2.72 & $330-348$ \\
54 & 27292 & 47839 & $2004 / 09 / 23$ & 0.899 & 1.36 & $152-161$ \\
55 & 27325 & 123806 & $2004 / 09 / 26$ & -0.692 & 2.33 & $170-180$ \\
\hline & & & & & Continued on next page \\
\hline
\end{tabular}


Table A.2 - continued from previous page

\begin{tabular}{|c|c|c|c|c|c|c|}
\hline$\#$ & Run & Snarl & Date & Cos $\theta_{\text {Zenith }}$ & VtxR(m) & Plane Range \\
\hline 56 & 27566 & 28709 & $2004 / 10 / 09$ & -0.353 & 0.765 & $137-145$ \\
57 & 27581 & 61928 & $2004 / 10 / 11$ & 0.824 & 1.5 & $320-333$ \\
58 & 27593 & 23945 & $2004 / 10 / 12$ & -0.63 & 1.09 & $305-323$ \\
59 & 27694 & 103569 & $2004 / 10 / 22$ & 0.628 & 2.88 & $371-399$ \\
60 & 27709 & 19610 & $2004 / 10 / 24$ & 0.609 & 0.738 & $34-47$ \\
61 & 27721 & 13243 & $2004 / 10 / 25$ & 0.748 & 3.59 & $312-332$ \\
62 & 27740 & 63058 & $2004 / 10 / 27$ & -0.478 & 2.98 & $403-417$ \\
63 & 27748 & 1328 & $2004 / 10 / 27$ & 0.859 & 1.93 & $112-123$ \\
64 & 27781 & 54144 & $2004 / 10 / 30$ & -0.0274 & 1.16 & $222-232$ \\
65 & 27858 & 51881 & $2004 / 11 / 06$ & 0.236 & 1.85 & $322-331$ \\
66 & 27885 & 28637 & $2004 / 11 / 09$ & -0.519 & 2.45 & $364-372$ \\
67 & 27950 & 94297 & $2004 / 11 / 15$ & 0.0821 & 1.97 & $238-284$ \\
68 & 28401 & 6778 & $2004 / 12 / 17$ & 0.437 & 1.85 & $382-394$ \\
69 & 28517 & 60935 & $2004 / 12 / 29$ & 0.267 & 1.18 & $30-38$ \\
70 & 28630 & 108505 & $2005 / 01 / 11$ & -0.403 & 1.98 & $73-100$ \\
71 & 28999 & 77389 & $2005 / 01 / 21$ & 0.156 & 0.709 & $265-289$ \\
72 & 29103 & 62927 & $2005 / 02 / 03$ & -0.0452 & 0.746 & $294-338$ \\
\hline
\end{tabular}

\section{A.2.2 Partially Contained Downward}

Table A.3: Selected Partially-Contained Downward Tracks

\begin{tabular}{|c|c|c|c|c|c|c|}
\hline$\#$ & Run & Snarl & Date & Cos $\theta_{\text {Zenith }}$ & $\operatorname{VtxR}(\mathrm{m})$ & Plane Range \\
\hline 1 & 18862 & 36180 & $2003 / 08 / 30$ & 0.416 & 1.57 & $467-483$ \\
2 & 20884 & 27361 & $2003 / 10 / 30$ & 0.367 & 2.34 & $49-60$ \\
3 & 22050 & 51358 & $2003 / 12 / 18$ & 0.653 & 1.27 & $354-425$ \\
4 & 22101 & 121177 & $2003 / 12 / 20$ & 0.382 & 1.03 & $119-142$ \\
5 & 22201 & 10289 & $2003 / 12 / 28$ & 0.106 & 1.83 & $13-24$ \\
6 & 22424 & 136567 & $2004 / 01 / 14$ & 0.898 & 2.31 & $74-94$ \\
\hline \multicolumn{7}{|c|}{} \\
\hline
\end{tabular}


Table A.3 - continued from previous page

\begin{tabular}{|c|c|c|c|c|c|c|}
\hline$\#$ & Run & Snarl & Date & Cos $\theta_{\text {Zenith }}$ & VtxR $(\mathrm{m})$ & Plane Range \\
\hline 7 & 22746 & 143907 & $2004 / 02 / 10$ & 0.274 & 1.5 & $383-392$ \\
8 & 22919 & 67340 & $2004 / 02 / 23$ & 0.209 & 1.61 & $62-177$ \\
9 & 22998 & 164927 & $2004 / 03 / 01$ & 0.192 & 1.64 & $237-247$ \\
10 & 23285 & 33554 & $2004 / 03 / 14$ & 0.377 & 1.44 & $296-415$ \\
11 & 23584 & 107525 & $2004 / 03 / 17$ & 0.665 & 2.7 & $227-248$ \\
12 & 24829 & 36847 & $2004 / 04 / 08$ & 0.365 & 1.42 & $372-395$ \\
13 & 24964 & 13099 & $2004 / 04 / 17$ & 0.474 & 0.687 & $388-401$ \\
14 & 25066 & 70672 & $2004 / 04 / 25$ & 0.0107 & 1.19 & $66-109$ \\
15 & 25627 & 47998 & $2004 / 06 / 08$ & 0.0982 & 1.06 & $352-368$ \\
16 & 25773 & 114723 & $2004 / 06 / 22$ & 0.377 & 2.72 & $167-201$ \\
17 & 26277 & 28086 & $2004 / 07 / 22$ & 0.27 & 2.36 & $187-331$ \\
18 & 27045 & 20111 & $2004 / 09 / 04$ & 0.012 & 1.64 & $18-329$ \\
19 & 27834 & 56265 & $2004 / 11 / 03$ & 0.748 & 1.28 & $337-348$ \\
20 & 27894 & 80994 & $2004 / 11 / 09$ & 0.329 & 1.58 & $225-248$ \\
21 & 28273 & 47784 & $2004 / 12 / 07$ & 0.0162 & 0.972 & $250-302$ \\
22 & 28535 & 65406 & $2004 / 12 / 31$ & 0.134 & 1.09 & $145-419$ \\
23 & 28600 & 34326 & $2005 / 01 / 07$ & 0.615 & 1.6 & $37-78$ \\
24 & 28609 & 97362 & $2005 / 01 / 08$ & 0.12 & 0.9 & $467-482$ \\
25 & 29103 & 35113 & $2005 / 02 / 03$ & 0.721 & 1.83 & $342-386$ \\
\hline
\end{tabular}

\section{A.2.3 Partially Contained Upward}

Table A.4: Selected Partially-Contained Upward Tracks

\begin{tabular}{|c|c|c|c|c|c|c|}
\hline$\#$ & Run & Snarl & Date & Cos $\theta_{\text {Zenith }}$ & $\operatorname{VtxR}(\mathrm{m})$ & Plane Range \\
\hline 1 & 18902 & 36351 & $2003 / 09 / 01$ & -0.456 & 0.809 & $115-176$ \\
2 & 20747 & 17532 & $2003 / 10 / 25$ & -0.762 & 1.15 & $121-139$ \\
3 & 21772 & 49880 & $2003 / 12 / 03$ & -0.214 & 0.9 & $285-312$ \\
4 & 21853 & 24825 & $2003 / 12 / 07$ & -0.361 & 1.28 & $118-128$ \\
\hline \multicolumn{1}{|c|}{ Continued on next page } \\
\hline
\end{tabular}


Table A.4 - continued from previous page

\begin{tabular}{|c|c|c|c|c|c|c|}
\hline$\#$ & Run & Snarl & Date & Cos $\theta_{\text {Zenith }}$ & VtxR $(\mathrm{m})$ & Plane Range \\
\hline 5 & 22336 & 22507 & $2004 / 01 / 09$ & -0.342 & 1.55 & $205-241$ \\
6 & 24874 & 37215 & $2004 / 04 / 13$ & -0.515 & 1.8 & $364-402$ \\
7 & 25189 & 77584 & $2004 / 05 / 05$ & -0.466 & 1.57 & $359-403$ \\
8 & 25304 & 10577 & $2004 / 05 / 12$ & -0.347 & 1.77 & $79-101$ \\
9 & 25988 & 89674 & $2004 / 07 / 11$ & -0.171 & 1.84 & $298-392$ \\
10 & 26830 & 27336 & $2004 / 08 / 16$ & -0.299 & 1.21 & $411-427$ \\
11 & 27503 & 9263 & $2004 / 10 / 05$ & -0.232 & 1.22 & $332-411$ \\
12 & 27724 & 85333 & $2004 / 10 / 26$ & -0.306 & 0.67 & $298-392$ \\
13 & 27879 & 79396 & $2004 / 11 / 08$ & -0.217 & 0.694 & $33-72$ \\
14 & 28226 & 69989 & $2004 / 12 / 02$ & -0.507 & 1.39 & $94-123$ \\
\hline
\end{tabular}




\section{Appendix B}

\section{Notes on Errors and Coverages}

Certain measurements made in this thesis involved many measurements for which the calculation of the error is not a trivial matter. This appendix will describe the computational methods by which the errors that are quoted in this document were determined.

\section{B.1 Double Ratio Coverage}

The double ratio measured in Chapter 7 and analyzed in Chapter 8 is called a double ratio as it is the fraction with the observed ratio of track event counts versus shower event counts as the numerator, and the expected ratio of track event count versus shower event count as the denominator. The initial approach to the measure double ratio error is to use Gaussian error propagation (assuming Poisson derived errors on the counted values).

The errors on the count of observed events will be the square root of that count. The same is true for the expected counts prior to scaling to the data exposure. The result of this error calculation is shown in Equation B.1.

$$
\begin{aligned}
N S h w_{\text {Data }} & =89 \\
N S h w_{\text {Exp }} & =88.0 \pm 1.0 \\
\operatorname{NTrk}_{\text {Data }} & =111 \\
\operatorname{NTrk}_{\text {Exp }} & =148.2 \pm 1.0
\end{aligned}
$$




$$
\frac{R_{\text {Trk } / S h w}^{\text {Data }}}{R_{\text {Trk } / S h w}^{E x p}}=0.75 \pm 0.11(68 \%) \pm 0.17(90 \%)
$$

The error expressed in B.1 on this double ratio is incomplete; it does not rigorously calculate the expected coverage of Poisson distributed values. The value is initially approximated analytically using the method outlined by Gehrels [102] and by Messier [103].

Gehrels devised a formulation to approximate the upper coverage of the ratio of two binomially distributed values from the same total sample. This can be applied to the given situation, assuming a total neutrino sample that is divide into two classes (showering and track-like ). The formulation in Equation 26 of Gehrels' paper yields $84.13 \%$ single-sided upper and lower coverages, equivalent to $68.27 \%$ double-sided coverages, in Equation B.2.

$$
\frac{R_{\text {Trk } / S h w}^{\text {Data }}}{R_{\text {Trk } / \text { Shw }}^{E x p}}=0.745_{-0.112}^{+0.130}(68 \%)_{-0.170}^{+0.218}(90 \%)
$$

This method only an approximation, devised in 1986 when the heavy computational effort involved in a Monte Carlo simulation was more constraining than it is today. Both of the above calculations were compared to the coverages measured with a toy Monte Carlo simulation of the random fluctuations of the numbers in B.1 The first approach takes the number of showers and tracks in the data and fluctuates them according to a Poisson distribution with a mean at the observed counts. The distributions of the resulting fluctuated counts are shown in Figures B.1 a and B.1.b. Figure B.1. shows the tracks versus the showers, demonstrating that there is no correlation between these two random values. Finally, the sum of the two random numbers averages to the sum of the means, and the RMS of this distribution is near the expected $R M S=\sqrt{\text { Mean }}$.

Each experiment casts random numbers of tracks and showers, which were used to express a double ratio. The distribution of these double ratios (Figure B.2 a) is used to determine the coverage of the measured double ratio. To measure the double-ratio coverage, $1 \times 10^{6}$ experiments were run, and all of the values were sorted and stored. The median of this distribution, which is the midpoint of the sorted double ratio values, is the same as the quoted measured value from Equation B.1. The limits of a $68.27 \%$ doublesided coverage are found to be the elements numbered 158,656 , and $10^{6}-158,656$ of the 

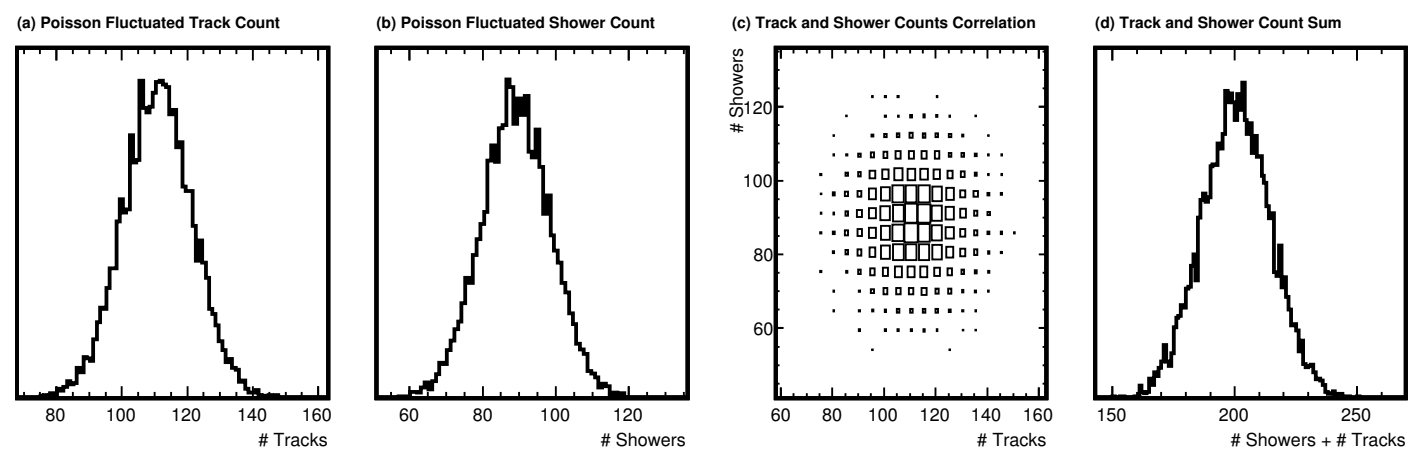

Figure B.1: Fluctuating the number of showers and tracks with a Poisson distribution. From left to right distributions of: track counts, of shower counts, track counts vs. shower counts and track + shower counts.

array of sorted double-ratio values. The resulting error values are shown in Equation B.3.
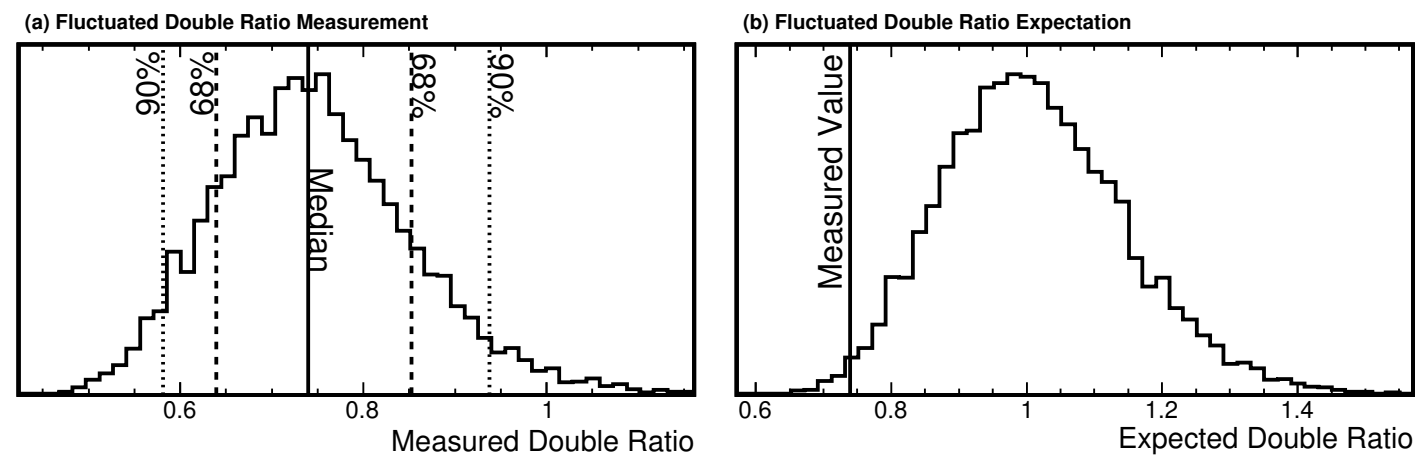

Figure B.2: Distributions of measured and expected double ratios obtained by fluctuating showers and tracks counts independently with a Poisson distribution.

$$
\frac{R_{\text {Trk } / \text { Shw }}^{\text {data }}}{R_{\text {Trk } / S h w}^{M C}}=0.745_{-0.099}^{+0.116}(68 \%)_{-0.155}^{+0.205}(90 \%)
$$

For the second plot in Figure B.2, the observed counts in the numerator of the double ratio were replaced with the expected counts. This gives an obvious double ratio of 1.0, and, by fluctuating the expected values in the numerator of the double ratio with Poisson distributions, a distribution of expected double ratio values is produced. Using this distribution, the median measured double ratio is excluded from the expected double 
ratio at $98.8 \%$ single-sided confidence level. This result means that these experiments disfavour a null oscillation hypothesis with a $98.8 \%$ confidence level.

A second experimental set is run as suggested by Messier 103, where the two classes of events are taken as subdivisions of a total number of events. The mean total number, which is just the sum of the number of tracks and showers, is then fluctuated by a Poisson distribution. This value is shown in Figure B.3a. The number of tracks is then fluctuated with a binomial distribution, where the total sample size equals the random summed event count, and the probability of the event being a track is $\frac{\text { NTracks }}{\text { NTracks+NShowers }}$. The fluctuated number of showers equals the random number of tracks subtracted from the random summed event count. The random tracks and showers are shown in Figures B.3b and B.3. F. Figure B.3 d demonstrates that there is no obvious correlation between these two random variables.
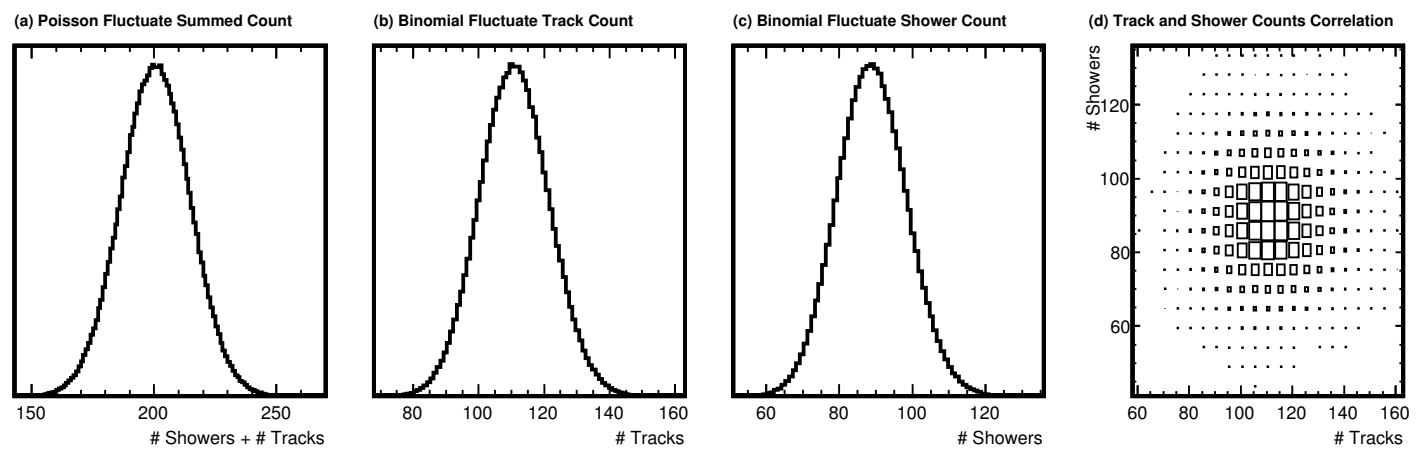

Figure B.3: Sum of shower and track counts fluctuated with a Poisson distribution, and fluctuated shower and track counts obtained with a binomial distribution from the resulting random summed count. From left to right distributions of: track + shower counts, track counts, shower counts, and number of tracks versus number of showers.

$$
\frac{R_{T r k / S h w}^{\text {data }}}{R_{T r k / S h w}^{M C}}=0.745_{-0.099}^{+0.116}(68 \%){ }_{-0.156}^{+0.203}(90 \%)
$$

These random shower and track counts are used to find the double ratio distribution, as shown Figure B.4a. This gives the double ratio coverage seen in Equation B.4, for which the error values are approximately the same as they had been when they were calculated with purely Poisson fluctuations. Also, the expected double ratio distribution 
(a) Fluctuated Double Ratio Measurement

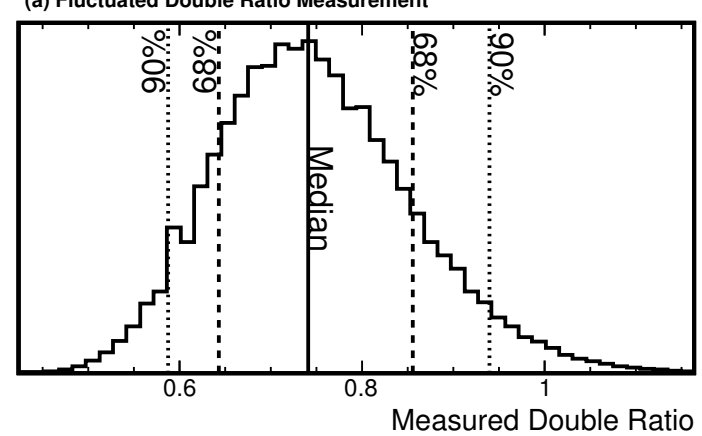

(b) Fluctuated Double Ratio Expectation

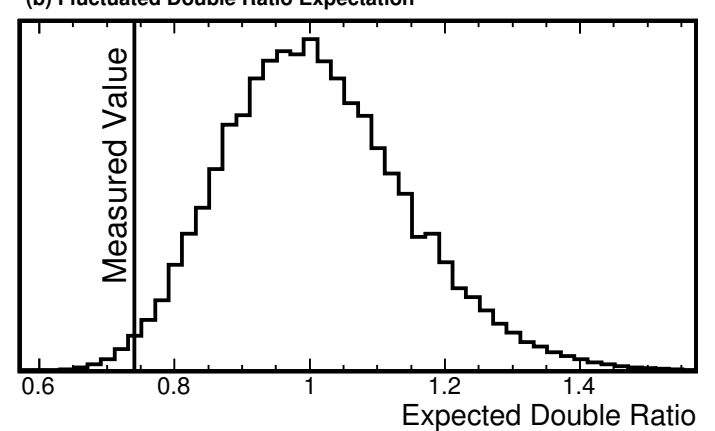

Figure B.4: Distributions of measured and expected double ratios resulting from fluctuating the sum of showers and tracks with a Poisson distribution, and obtaining the number of showers and tracks from a binomial distribution.

in Figure B.4 yields approximately the $98.7 \%$ confidence to disfavor the null oscillation hypothesis.

For both the Poisson and Poisson+Binomial experimental toy Monte Carlo sets, the expected counts in the denominator of the double ratio have a small margin of error, and have therefore not been fluctuated. The Monte Carlo simulation set is scaled down by $\sim 1 / 200$, and will thus have a smaller fractional statistical error than the data set by a factor of $\sqrt{200} \sim 14.2$. To see how this might affect the measurement, both the Poisson and Poisson+Binomial experiments were run again including the fluctuation of the Monte Carlo counts in the denominator of the double ratio. Both situation still yield an upper limit of +0.116 and a lower limit of -0.099 for $68 \%$ coverage and +0.203 by -0.156 for $90 \%$ coverage. Applying Gaussian fluctuations to the denominator in the double ratio has the effect of smoothing the Poisson spikes in the measured and expected double ratio distributions.

The number of significant digits selected to display the full double ratio (to the thousandth digit) is based on the small fluctuation of the error of this digit. When running multiple experiments, the error of the statistical error extends to the thousandth digit. In this appendix, the double ratio has been expressed as $\mathrm{R}=0.745$ due to the belief from the $\mathrm{MC}$ fluctuations that three significant digits are appropriate. Other experiments that have measured the atmospheric neutrino double ratio have typically used significant digits to the hundredth digit [44]. In order to present a value which may be easily compared to these other experiments, the double ratio is quoted in the 
body of this thesis to the hundredth digit $(\mathrm{R}=0.74)$.

These experiments demonstrate that the coverage for the double ratio accurately modeled with a toy Monte Carlo using the independent Poisson fluctuations of the showers and tracks. The method outlined by Gehrels [102] over-estimates the coverage region by $\sim 10 \%$, which is consistent with Messier's results when compared to Gehrels' method. The second Monte Carlo method, employing both Poisson and Binomial distributions to fluctuate the counts, is slightly more CPU intensive, and arrives at the same answer as the first Monte Carlo method that employs only Poisson distributions to fluctuate data. The double ratio expressed in this thesis will use the coverages determined by the first Monte Carlo method.

\section{B.2 Analytic Maximization of Likelihood with Scaled Con- tributions}

The negative log likelihood function that fits an expected distribution to an observed distribution for Poisson binned probability is expressed generally in Equation B.5, where the fit is performer over $N B$ bins, and applying $N P$ penalty factors. The goal of a likelihood fit is to minimize this equation, which means that the derivative of this function with respect to any given parameter $(S)$ can be set to zero and solved to obtain the best fit of that parameter. One would expect that only $E_{i}$ and $\alpha$ would depend on $S$, and thus the minimization is solved generally with Equation B.6.

$$
\begin{aligned}
\text { LLHood } & =\sum_{i}^{N B}\left(E_{i}-O_{i} \ln E_{i}\right)+\sum_{j}^{N P} \frac{\alpha_{j}^{2}}{2 \sigma_{j}^{2}} \\
\frac{\partial}{\partial S} \text { LLHood } & =\sum_{i}\left(\frac{\partial E_{i}}{\partial S}+\frac{O_{i}}{E_{i}} \frac{\partial E_{i}}{\partial S}\right)+\sum_{j}^{N P}\left(\frac{\alpha_{j}}{\sigma_{j}^{2}} \frac{\partial \alpha_{j}}{\partial S}\right) \\
& =\sum_{i}^{N B}\left[\frac{\partial E_{i}}{\partial S}\left(1-\frac{O_{i}}{E_{i}}\right)\right]+\sum_{j}^{N P}\left(\frac{\alpha_{j}}{\sigma_{j}^{2}} \frac{\partial \alpha_{j}}{\partial S}\right)=0
\end{aligned}
$$

In practice, [B.6 is not always simple to solve, given that the parameter can have a variety of non-linear expressions in the $E_{i}$ or $\alpha_{j}$ terms. The situation analyzed has a fairly simple approach, where a scale factor is applied to one of the contributing factors 
in $E_{i}$, and the scale factor penalty is defined as $\alpha=S-S^{0}$. The optimal scale factor is usually $S^{0}=1.0$, and the term $\frac{\partial \alpha}{\partial S}=1$.

The simplest possibility is to first ignore the penalty term, and then say that $E_{i}=$ $S_{a t m} E_{i \nu}$, leading to $\frac{\partial E_{i}}{\partial S_{a t m}}=E_{i \nu}$. If this is the case, then Equation B.6 is solved as $S_{a t m}=\frac{\sum O_{i}}{\sum E_{i}}$. In this case states, when scaling an expected distribution to best match an observed distribution, the shape doesn't matter at all. The best fit scale is just the ratio of the total counts.

If the penalty term is put back into the equation, but $E_{i}=S_{a t m} E_{i \nu}$ remains, the solution for $S_{a t m}$ from Equation B.6 is expressed in the equation that is quadratic in $S_{a t m}$ in B.7. The linear term here will be positive if $\sum E_{i \nu}>\frac{S_{a t m}^{0}}{\sigma^{2}}$, meaning that uncertainty would be no bigger than the size of the sample. If the linear term is positive, then there can only be a single positive solution. Also, $\left(\sum E_{i \nu}-\frac{1.0}{\sigma^{2}}\right)^{2}+4 \frac{\sum O_{i}}{\sigma^{2}}$ must be greater than zero, so the quadratic equation will have a real solution.

$$
\frac{S_{a t m}^{2}}{\sigma^{2}}+S_{a t m}\left(\sum_{i}^{N B} E_{i \nu}-\frac{S_{a t m}^{0}}{\sigma^{2}}\right)+\sum_{i}^{N B} O_{i}=0
$$

Next a background term is added to the expected content, or the $\mathrm{i}^{\text {th }}$ bin content would be $E_{i}=S_{a t m} E_{i \nu}+B_{i}$. In this case, the partial term $\frac{\partial E_{i}}{\partial S_{a t m}}$ doesn't change, but $S_{a t m}$ can no longer be extracted from the second factor in the sum. The resulting equation is expressed in B.8. To simplify this equation, the denominator of the right hand side of the equation can be eliminated by multiplying both sides of the equation by the product of all possible denominators. This results in a polynomial that has an order of $N$ Bins +1 in Equation [B.9] where $E_{\nu}=\sum_{i}^{N B} E_{i \nu}$. If the penalty term is removed, the polynomial becomes of the order of NBins.

$$
\begin{aligned}
\sum_{i}^{N B}\left(E_{i \nu}\right)+\frac{S_{a t m}-S_{a t m}^{0}}{\sigma^{2}} & =\sum_{i}^{N B}\left(\frac{O_{i} E_{i \nu}}{S_{a t m} E_{i \nu}+B_{i}}\right) \\
\left(E_{\nu}+\frac{S_{a t m}-S_{a t m}^{0}}{\sigma^{2}}\right) \prod_{k}^{N B}\left(S_{a t m} E_{k \nu}+B_{k}\right) & =\sum_{i}^{N B}\left[O_{i} E_{i \nu} \prod_{k \neq i}^{N B}\left(S_{a t m} E_{k \nu}+B_{k}\right)\right]
\end{aligned}
$$


The $\prod\left(S_{a t m} E_{i \nu}+B_{i}\right)$ product terms on the each side of Equation B.9 can be generalized to polynomials in $S_{a t m}$ using a Pascal triangle [104]. Equation B.10]demonstrates a progression of converting $\prod_{i}^{N}\left(A_{i} x+B_{i}\right)$ into an $N^{t h}$ order polynomial in $x$ with a Pascal triangle. The product of $S_{a t m} E_{i \nu}+B_{i}$, iterated over NB terms, can be expressed as an $N B^{\text {th }}$ order polynomial, or $\sum_{i}^{N B}\left(C_{i} S_{a t m}{ }^{i}\right)$. Using an expansion that is analogous to the one in Equation B.10 produces $C_{0}=\prod_{i}^{N B} B_{i}, C_{N B}=\prod_{i}^{N B} E_{i \nu}$, and inner coefficients dictated by the triangle's progression.

$$
\begin{gathered}
x A_{0}+B_{0} \\
x^{N} \prod_{i}^{N}\left(A_{i}\right) \\
x^{2} A_{0} A_{1}+\quad x\left(A_{0} B_{1}+A_{1} B_{0}\right)+B_{0} B_{1} \\
\ldots .
\end{gathered}
$$

The $\sum_{i}^{N B}\left[O_{i} E_{i \nu} \prod_{k \neq i}^{N B}\left(S_{a t m} E_{k \nu}+B_{k}\right)\right]$ term in Equation B.9 can also be converted into a polynomial in $S_{a t m}$ using a series of Pascal triangle expansions, and expressed as $\sum_{i}^{N B}\left(D_{i} S_{a t m}{ }^{i}\right)$. The product is only iterated over $N B-1$ terms, and so $D_{N}=0$. The outer terms are $D_{N-1}=\sum_{i}^{N B}\left[E_{i \nu} O_{i} \prod_{j \neq i}^{N B}\left(E_{j \nu}\right)\right]$ and $D_{O}=\sum_{i}^{N B}\left[E_{i \nu} O_{i} \prod_{j \neq i}^{N B}\left(B_{j}\right)\right]$, and the inner terms are found through the triangle progression. Equation B.9 is converted to the polynomial of order $N B+1$ expressed in Equation B.11. This is algebraically simplified to Equation B.12, where the $C_{i-1}$ term arises from the application of the penalty factor. The $C_{-1}$ coefficient from the point where $i=0$ in the sum is treated as 0 . If the penalty term is removed by $\sigma \rightarrow \infty$, then Equation B.12 simplifies to B.13 which is a polynomial of order $N B$.

$$
\begin{aligned}
\left(E_{\nu}+\frac{S_{a t m}-S_{a t m}^{0}}{\sigma^{2}}\right) \sum_{i}^{N B}\left(C_{i} S_{a t m}{ }^{i}\right)=\sum_{i}^{N B}\left(D_{i} S_{a t m}{ }^{i}\right) & \\
\frac{C_{N}}{\sigma^{2}} S_{a t m}{ }^{N B+1}+\sum_{i=0}^{N B}\left[C_{i} E_{\nu}+\frac{C_{i-1}-C_{i} S_{a t m}^{0}}{\sigma^{2}}-D_{i}\right] S_{a t m}{ }^{i} & =0 \\
\sum_{i=0}^{N B}\left(C_{i} E_{\nu}-D_{i}\right) S_{a t m}{ }^{i} & =0
\end{aligned}
$$

The situation in Section 8.3 only fits for two bins: the number of showers and the number of tracks. This makes the minimization of the negative log-likelihood into the 
solution of a $2^{\text {nd }}$ order(quadratic) polynomial for an unpenalized scale factor, or a $3^{\text {rd }}$ order (cubic) polynomial for a penalized scale factor. The quadratic polynomial is solvable analytically by completing squares. The cubic polynomial is solved with the method outlined by Cardano[105. Higher order polynomials can be solved numerically with functions from the GNU Scientific Library [106]. Using $E_{0}=S_{a t m} E_{S \nu}+B_{S}$ and $E_{1}=S_{a t m} E_{T \nu}+B_{T}$, Equation B.12 becomes Equation B.14.

$$
\begin{aligned}
0=S_{a t m}^{3} & \times\left(\frac{E_{S \nu} E_{T \nu}}{\sigma^{2}}\right) \\
+S_{a t m}^{2} & \times\left[E_{S \nu} E_{T \nu}\left(E_{S \nu}+E_{T \nu}\right)+\frac{E_{S \nu} B_{T}+E_{T \nu} B_{S}}{\sigma^{2}}\right] \\
+S_{a t m} & \times\left[\left(E_{S \nu}+E_{T \nu}\right)\left(E_{S \nu} B_{T}+E_{T \nu} B_{S}\right)+\frac{B_{S} B_{T}}{\sigma^{2}}-E_{S \nu} E_{T \nu}\left(O_{S}+O_{T}\right)\right] \\
+1 & \times\left[\left(E_{S \nu}+E_{T \nu}\right) B_{S} B_{T}-\left(O_{S} E_{S \nu} B_{T}+O_{T} E_{T \nu} B_{S}\right)\right]
\end{aligned}
$$

If the penalty term vanishes $(\sigma \rightarrow \infty)$, this becomes much simpler, as the cubic term becomes zero and the rest of the terms simplify. Both solutions are used when approaching the flux scale solution in Section 8.3. This reduces the processing time significantly for the negative log likelihood method by not having to numerically minimize for a scale factor at each oscillation grid point.

A final reflection on this issue considers a more fully generalized situation with multiple distributions, each with their own scale factor. The expectation of each bin is a sum of $N D$ distinct distributions. Each distribution is assigned its own scale factor $\left(S_{k}\right)$, and each scale factor is penalized from optimal $\left(S_{k}^{0}\right)$ with some uncertainty $\left(\sigma_{k}\right)$. The sum of expected events for a distribution is expressed as $E_{k}=\sum_{\imath}^{N B} E_{\imath k}$. The partial derivative with respect to an $S_{k}$ term is expressed in Equation B.15, and would have to equal zero for all values of $k$ in order to fully minimize the negative log likelihood function.

$$
E_{k}+\frac{S_{k}-S_{k}^{0}}{\sigma_{k}^{2}}=\sum_{\imath}^{N B}\left(\frac{O_{\imath} E_{\imath k}}{\sum_{\jmath}^{N D}\left(S_{\jmath} E_{\imath \jmath}\right)}\right)
$$

In the same vein as the method with a single scale factor with a background contribution, both sides of this equation can be multiplied by the least common denominator of the right hand side of Equation $\mathrm{B} .15\left(\prod_{\imath}^{N B}\left[\sum_{\jmath}^{N D}\left(S_{\jmath} E_{\imath \jmath}\right)\right]\right)$, where NB is the number 
of bins and ND is the number of contributing distributions. Equation [B.16] is a general solution for this minimization when it is true for all $k$.

$$
\left(E_{k}+\frac{S_{k}-S_{k}^{0}}{\sigma_{k}^{2}}\right) \times \prod_{\imath}^{N B}\left(\sum_{\jmath}^{N D}\left(S_{\jmath} E_{\imath \jmath}\right)\right)=\sum_{\imath}^{N B}\left[\left(O_{\imath} E_{\imath k}\right) \prod_{\jmath \neq \imath}^{N B}\left(\sum_{\ell}^{N D}\left(S_{\ell} E_{\jmath}\right)\right)\right]
$$

The product term $\prod_{\imath}^{N B}\left(\sum_{\jmath}^{N D}\left(S_{\jmath} E_{\imath \jmath}\right)\right)$ can be converted to a polynomial of the order $N B$, such that each term has some coefficient and a combinatorial product of $S_{\jmath}$ terms. The combinatorial product of $S_{\jmath}$ will have a summed set of powers equal to the total number of distribution bins. Equation B.17 demonstrates this expansion, where the combinatorial summation is iterated over all combinations of $P_{\imath \mathrm{Comb}}$ such that the sum of all terms iterated over $\jmath$ equals $N B$. The $C_{\imath \text { Comb }}$ coefficient can be determined with a Pascal's simplex, which is analogous to the Pascal's triangle for the product of multinomial equations.

$$
\begin{aligned}
\prod_{\imath}^{N B}\left(\sum_{\jmath}^{N D}\left(S_{\jmath} E_{\imath \jmath}\right)\right) & =\sum_{\imath}^{N B}\left[\sum_{C o m b} C_{\imath \text { Comb }} \prod_{\jmath}^{N D}\left(S_{\jmath} P_{\imath \text { Comb }_{\jmath}}\right)\right] \\
\sum_{C o m b} & \Rightarrow \text { Combinatorics of } P_{\imath \text { Comb } \mathrm{\jmath}} \text { in }[0, N D] \\
C_{\imath C o m b}=0 & \text { if } \sum_{\jmath}^{N D} P_{\imath C o m b \jmath} \neq N D
\end{aligned}
$$

After the expansion in Equation B.17 is applied to both of the product terms in Equation B.16 the solution is a system of $N D$ polynomial equations (one for each $k$ ). Each polynomial equation is of order $N$ Bins +1 in $S_{k}$, or NBins if $\sigma_{k} \rightarrow \infty$. Any kind of closed analytic solution to the system of polynomials is not easily achieved, and in most cases would require a numerical solution. Devising a numerical solution would be difficult because building a full set of coefficients from sets of Pascal simplexes would be computationally intensive.

Another approach to fitting contributions from multiple expected distributions to a single observed distribution by Barlow et al. [107] uses Minuit minimization to solve for the relative scaled contributions of the expected distributions. Their approach doesn't apply penalty terms for scale factors, but can be modified to do so. This method is also 
limiting, since it will find a single minimum near 1.0 for all scale factors. The generic polynomial approach would find all real roots, and identify if multiple physical minima exist. A fully implemented polynomial method could more insight in to the matter addressed by Barlow et al., and warrants further investigation. 


\section{References}

[1] John G. Learned. The atmospheric neutrino anomaly: Muon neutrino disappearance. 2000, arXiv:hep-ex/0007056.

[2] G. D. Barr, T. K. Gaisser, P. Lipari, Simon Robbins, and T. Stanev. A threedimensional calculation of atmospheric neutrinos. Phys. Rev., D70:023006, 2004, astro-ph/0403630.

[3] W. W. M. Allison et al. Neutrino oscillation effects in soudan-2 upward-stopping muons. Phys. Rev., D72:052005, 2005, hep-ex/0507068.

[4] P. Adamson et al. First observations of separated atmospheric nu/mu and anti- nu/mu events in the minos detector. Phys. Rev., D73:072002, 2006, hep$\mathrm{ex} / 0512036$.

[5] G. Barr. Three-dimensional calculation of atmospheric neutrino. Phys. Rev. D, 70:89-95, 2004.

[6] G. D. Barr, T. K. Gaisser, S. Robbins, and Todor Stanev. Uncertainties in atmospheric neutrino fluxes. Phys. Rev., D74:094009, 2006, astro-ph/0611266.

[7] A. Einstein. of elementary particles. Prog. Theor. Phys., 28:870, 1905.

[8] J. Chadwick. Possible existence of a neutron. Nature, 129:312, 1932.

[9] W. Pauli. On the earlier and more recent history of the neutrino. Camb. Monogr. Part. Phys. Nucl. Phys. Cosmol., 14:1-22, 2000.

[10] Nockolas Solomey. The Elusive Neutrino. Scientific American Library, 1997. 
[11] B. Pontecorvo. Inverse beta process. Cambridge Monogr. Part. Phys. Nucl. Phys. Cosmol., 1:25-31, 1991.

[12] Raymond Davis. Attempt to detect the antineutrinos from a nuclear reactor by the $c^{37}\left(\nu[\right.$ over $\left.], e^{-}\right) a^{37}$ reaction. Phys. Rev., 97(3):766-769, Feb 1955.

[13] W. F. Hornyak, T. Lauritsen, P. Morrison, and W. A. Fowler. Energy levels of light nuclei. iii. Rev. Mod. Phys., 22(4):291-372, Oct 1950.

[14] L. M. Langer and R. J. D. Moffat. The beta-spectrum of tritium and the mass of the neutrino. Phys. Rev., 88(4):689-694, Nov 1952.

[15] Seth H. Neddermeyer and Carl D. Anderson. Nature of cosmic-ray particles. Rev. Mod. Phys., 11(3-4):191-207, Jul 1939.

[16] M. Schwartz. Feasibility of using high-energy neutrinos to study the weak interactions. Phys. Rev. Lett., 4(6):306-307, Mar 1960.

[17] B. Pontecorvo. Electron and muon neutrinos. Sov. Phys. JETP, 10:1236-1240, 1960.

[18] G. Danby, J-M. Gaillard, K. Goulianos, L. M. Lederman, N. Mistry, M. Schwartz, and J. Steinberger. Observation of high-energy neutrino reactions and the existence of two kinds of neutrinos. Phys. Rev. Lett., 9(1):36-44, Jul 1962.

[19] W.-M. Yao et al. Review of Particle Physics. Journal of Physics G, 33:1+, 2006.

[20] Kai Zuber, editor. Neutrino Physics. Institute of Physics Publishing, 2004.

[21] Halzen and Martin. Quarks and Leptons. John Wiley and Sons, 1984.

[22] Z. Maki, M. Nakagawa, and S. Sakata. Remarks on the unified model of elementary particles. Prog. Theor. Phys., 28:870, 1962.

[23] B. Pontecorvo. Neutrino experiments and the question of leptonic-charge conservation. Sov. Phys. JETP, 26:984-988, 1968.

[24] Marek Zralek. From kaons to neutrinos: Quantum mechanics of particle oscillations. Acta Phys. Polon., B29:3925-3956, 1998, hep-ph/9810543. 
[25] J. Bahcall. Neutrino Astrophysics. Cambridge University Press, 1989.

[26] John N. Bahcall and M. H. Pinsonneault. Standard solar models, with and without helium diffusion and the solar neutrino problem. Rev. Mod. Phys., 64:885-926, 1992.

[27] Raymond Davis. Solar neutrinos. ii. experimental. Phys. Rev. Lett., 12(11):303305, Mar 1964.

[28] Jr. Davis, Raymond, Don S. Harmer, and Kenneth C. Hoffman. Search for neutrinos from the sun. Phys. Rev. Lett., 20:1205-1209, 1968.

[29] Dzh. N. Abdurashitov et al. Results from sage. Phys. Lett., B328:234-248, 1994.

[30] P. Anselmann et al. Implications of the gallex determination of the solar neutrino flux. Phys. Lett., B285:390-397, 1992.

[31] Y. Suzuki. Kamiokande solar neutrino results. Nucl. Phys. Proc. Suppl., 38:54-59, 1995.

[32] S. Fukuda et al. Determination of solar neutrino oscillation parameters using 1496 days of super-kamiokande-i data. Phys. Lett., B539:179-187, 2002, hepex/0205075.

[33] B. Aharmim et al. Electron energy spectra, fluxes, and day-night asymmetries of b-8 solar neutrinos from the 391-day salt phase sno data set. Phys. Rev., C72:055502, 2005, nucl-ex/0502021.

[34] L. Wolfenstein. Neutrino oscillations in matter. Phys. Rev., D17:2369, 1978.

[35] S. P. Mikheev and A. Yu. Smirnov. Resonance enhancement of oscillations in matter and solar neutrino spectroscopy. Sov. J. Nucl. Phys., 42:913-917, 1985.

[36] John N. Bahcall, M. C. Gonzalez-Garcia, and Carlos Pena-Garay. Global analysis of solar neutrino oscillations including sno cc measurement. JHEP, 08:014, 2001, hep-ph/0106258.

[37] M. Nakahata. Future solar neutrino experiments. Nucl. Phys. Proc. Suppl., 145:23-28, 2005. 
[38] G. Battistoni, A. Ferrari, T. Montaruli, and P. R. Sala. The fluka atmospheric neutrino flux calculation. Astropart. Phys., 19:269-290, 2003, hep-ph/0207035.

[39] R. Becker-Szendy et al. The electron-neutrino and muon-neutrino content of the atmospheric flux. Phys. Rev., D46:3720-3724, 1992.

[40] K. S. Hirata et al. Observation of a small atmospheric $\nu_{\mu} / \nu_{e}$ ratio in kamiokande. Phys. Lett., B280:146-152, 1992.

[41] M. Aglietta et al. Experimental study of atmospheric neutrino flux in the nusex experiment. Europhys. Lett., 8:611-614, 1989.

[42] Christoph Berger et al. A study of atmospheric neutrino oscillations in the frejus experiment. Phys. Lett., B245:305-310, 1990.

[43] W. W. M. Allison et al. Measurement of the atmospheric neutrino flavour composition in soudan-2. Phys. Lett., B391:491-500, 1997, hep-ex/9611007.

[44] W. Anthony Mann. Atmospheric neutrinos and the oscillations bonanza. 1999, hep-ex/9912007.

[45] Y. Ashie et al. A measurement of atmospheric neutrino oscillation parameters by super-kamiokande i. Phys. Rev., D71:112005, 2005, hep-ex/0501064.

[46] Y. Ashie et al. Evidence for an oscillatory signature in atmospheric neutrino oscillation. Phys. Rev. Lett., 93:101801, 2004, hep-ex/0404034.

[47] S. Fukuda et al. Tau neutrinos favored over sterile neutrinos in atmospheric muon neutrino oscillations. Phys. Rev. Lett., 85:3999-4003, 2000, hep-ex/0009001.

[48] H. Kwon et al. Search for neutrino oscillations at a fission reactor. Phys. Rev., D24:1097-1111, 1981.

[49] G. Zacek et al. Neutrino oscillation experiments at the gosgen nuclear power reactor. Phys. Rev., D34:2621-2636, 1986.

[50] Y. Declais et al. Search for neutrino oscillations at 15-meters, 40-meters, and 95-meters from a nuclear power reactor at bugey. Nucl. Phys., B434:503-534, 1995. 
[51] Boehm and Vogel. Physics of Massive Neutrinos. Cambridge University Press, 1987.

[52] M. Apollonio et al. Search for neutrino oscillations on a long base-line at the chooz nuclear power station. Eur. Phys. J., C27:331-374, 2003, hep-ex/0301017.

[53] M. Apollonio et al. Limits on neutrino oscillations from the chooz experiment. Phys. Lett., B466:415-430, 1999, hep-ex/9907037.

[54] A. Piepke. Kamland: A reactor neutrino experiment testing the solar neutrino anomaly. Nucl. Phys. Proc. Suppl., 91:99-104, 2001.

[55] K. Eguchi et al. First results from kamland: Evidence for reactor anti- neutrino disappearance. Phys. Rev. Lett., 90:021802, 2003, hep-ex/0212021.

[56] T. Araki et al. Measurement of neutrino oscillation with kamland: Evidence of spectral distortion. Phys. Rev. Lett., 94:081801, 2005, hep-ex/0406035.

[57] C. Athanassopoulos et al. Evidence for anti-nu/mu -i, anti-nu/e oscillation from the lsnd experiment at the los alamos meson physics facility. Phys. Rev. Lett., 77:3082-3085, 1996, nucl-ex/9605003.

[58] A. Aguilar et al. Evidence for neutrino oscillations from the observation of antinu/e appearance in a anti-nu/mu beam. Phys. Rev., D64:112007, 2001, hepex/0104049.

[59] B. Armbruster et al. Upper limits for neutrino oscillations anti-nu/mu -i antinu/e from muon decay at rest. Phys. Rev., D65:112001, 2002, hep-ex/0203021.

[60] A. Romosan et al. A high statistics search for nu/mu (anti-nu/mu) - i nu/e (anti$\mathrm{nu} / \mathrm{e})$ oscillations in the small mixing angle regime. Phys. Rev. Lett., 78:2912-2915, 1997, hep-ex/9611013.

[61] Andrew Bazarko. Miniboone: Status of the booster neutrino experiment. Nucl. Phys. Proc. Suppl., 91:210-215, 2001, hep-ex/0009056.

[62] A. Suzuki et al. Design, construction, and operation of scifi tracking detector for k2k experiment. Nucl. Instrum. Meth., A453:165-176, 2000, hep-ex/0004024. 
[63] E. Aliu et al. Evidence for muon neutrino oscillation in an accelerator- based experiment. Phys. Rev. Lett., 94:081802, 2005, hep-ex/0411038.

[64] MINOS collaboration. The MINOS Detectors Technical Design Report. NuMI 337, October 1998.

[65] Sacha E. Kopp. The numi neutrino beam at fermilab. 2005, physics/0508001.

[66] Et Al. Indurthy D. Ion chambers for monitoring the numi neutrino beam at fnal. NuMI 1028, May 2004.

[67] Drake G. Dawson J. Nelson C. Overview of the front end electronics for the near detector. NuMI 628, January 1999.

[68] Caius L. F. Howcroft. Atmospheric neutrinos in the minos far detector. FERMILAB-THESIS-2004-71.

[69] David Boehnlein. Systematics errors in the steel thickness of the minos far detector. NuMI 1088, May 2005.

[70] C. et al. Andreopoulos. Results from the minos far detector four plane prototype. NuMI 994, November 2001.

[71] MINOS Collaboration. Proposal for a cosmic ray veto shield for minos. NuMI 1037, June 2004.

[72] N. Tagg et al. Performance of hamamatsu 64-anode photomultipliers for use with wavelength-shifting optical fibres. Nucl. Instrum. Meth., A539:668-678, 2005, physics/0408055.

[73] S. Avvakumov et al. Spontaneous light emission from fibers in minos. Nucl. Instrum. Meth., A545:145-155, 2005.

[74] J. Oliver et al. Design and performance of the readout system of the minos far detector. IEEE Trans. Nucl. Sci., 51:2193-2195, 2004.

[75] N. et. al. Felt. MINOS far detector electronics. User Manual, October 2001. 
[76] A. Belias et al. The minos data acquisition system. IEEE Trans. Nucl. Sci., 51:451-455, 2004.

[77] R. Zweber. Personal communication via DGB Huddle, May 2007.

[78] Stanislaw Ulam. Adventures of a Mathematician. University of California Press, 1991.

[79] T. Gaisser. Cosmic rays and particle physics. Cambridge University Press, 1990.

[80] Susan Marie Kasahara. A study of cosmic ray composition in the knee region using multiple muon events in the soudan-2 detector. UMI-97-21625.

[81] H. Gallagher. The neugen neutrino event generator. Nucl. Phys. Proc. Suppl., 112:188-194, 2002.

[82] CERN. http://wwwasd.web.cern.ch/wwwasd/geant/

[83] C. Zeitnitz and T. A. Gabriel. The GEANT - CALOR interface and benchmark calculations of zeus test calorimeters. Nucl. Instrum. Meth., A349:106-111, 1994.

[84] H. Fesefeldt. The simulation of hadronic showers: Physics and applications. PITHA-85-02.

[85] R. Merenyi, W. A. Mann, T. Kafka, W. Leeson, B. Saitta, J. Schneps, M. Derrick, and B. Musgrave. Determination of pion intranuclear rescattering rates in $\nu_{\mu}-n e$ versus $\nu_{\mu}-d$ interactions for the atmospheric $\nu$ flux. Phys. Rev. D, 45(3):743-751, Feb 1992.

[86] Robert Hatcher. Gminos and detsim status. NuMI 1238, November 2005.

[87] J.H. Birks. The theory and practice of scintillation counting. Pergamon Press, 1964.

[88] Andy Blake. Low level processing of atmospheric neutrino data. NuMI 1498, February 2006.

[89] Ryan Nichol. Calibration of the minos detectors. FERMILAB-THESIS-2003-41. 
[90] Christopher B. Smith. Calibration of the minos detectors and extraction of neutrino oscillation parameters. FERMILAB-THESIS-2002-58.

[91] Jeffrey J. Hartnell. Measurement of the calorimetric energy scale in minos. FERMILAB-THESIS-2005-51.

[92] P. Adamson et al. The minos light injection calibration system. Nucl. Instrum. Meth., A492:325-343, 2002, hep-ex/0204021.

[93] Andrew Blake. A study of atmospheric neutrino oscillations in the minos far detector. FERMILAB-THESIS-2005-77.

[94] Mark Thomson. Altdemux. NuMI 2535, December 2006.

[95] D. G. Michael et al. Observation of muon neutrino disappearance with the MINOS detectors and the NuMI neutrino beam. Phys. Rev. Lett., 97:191801, 2006, hepex/0607088.

[96] CP Ward. Cambridge monte carlo generation

- http://www.hep.phy.cam.ac.uk/minos/group/mc/mc_nov04_map201.html.

[97] M. Ambrosio et al. Seasonal variations in the underground muon intensity as seen by macro. Astropart. Phys., 7:109-124, 1997.

[98] Pat Ward. Simulation of neutron background to atmospheric neutrino analyses. NuMI 1085, May 2005.

[99] Mayly C. Sanchez et al. Observation of atmospheric neutrino oscillations in soudan 2. Phys. Rev., D68:113004, 2003, hep-ex/0307069.

[100] Herbert Goldstein. Classical Mechanics. Addison-Wesley Publishing, 1980.

[101] B. Rossi. High-energy Particles. Prentice Hall, 1952.

[102] N Gehrels. Confidence limits for small numbers of events in astrophysical data. Astrophys. J., 303:336-346, 1986.

[103] Mark Messier. Poisson errors on events ratios. NuMI 2299, October 2006. 
[104] Blaise Pascal. Trait du triangle arithmtique. 1654.

[105] Gerolamo Cardano. Ars Magna. 1545.

[106] et al M. Galassi. http://www.gnu.org/software/gsl/index.html.

[107] Roger J. Barlow and Christine Beeston. Fitting using finite monte carlo samples. Comput. Phys. Commun., 77:219-228, 1993. 